\title{
MRI for personalised treatment in oesophageal cancer
}

Citation for published version (APA):

Vollenbrock, S. (2020). MRI for personalised treatment in oesophageal cancer. [Doctoral Thesis, Maastricht University]. Ipskamp Printing BV. https://doi.org/10.26481/dis.20201209sv

Document status and date:

Published: 01/01/2020

DOI:

10.26481/dis.20201209sv

Document Version:

Publisher's PDF, also known as Version of record

\section{Please check the document version of this publication:}

- A submitted manuscript is the version of the article upon submission and before peer-review. There can be important differences between the submitted version and the official published version of record.

People interested in the research are advised to contact the author for the final version of the publication, or visit the DOI to the publisher's website.

- The final author version and the galley proof are versions of the publication after peer review.

- The final published version features the final layout of the paper including the volume, issue and page numbers.

Link to publication

\footnotetext{
General rights rights.

- You may freely distribute the URL identifying the publication in the public portal. please follow below link for the End User Agreement:

www.umlib.nl/taverne-license

Take down policy

If you believe that this document breaches copyright please contact us at:

repository@maastrichtuniversity.nl

providing details and we will investigate your claim.
}

Copyright and moral rights for the publications made accessible in the public portal are retained by the authors and/or other copyright owners and it is a condition of accessing publications that users recognise and abide by the legal requirements associated with these

- Users may download and print one copy of any publication from the public portal for the purpose of private study or research.

- You may not further distribute the material or use it for any profit-making activity or commercial gain

If the publication is distributed under the terms of Article $25 \mathrm{fa}$ of the Dutch Copyright Act, indicated by the "Taverne" license above, 


\section{MRI \\ FOR PERSONALISED TREATMENT \\ in \\ OESOPHAGEAL CANCER}

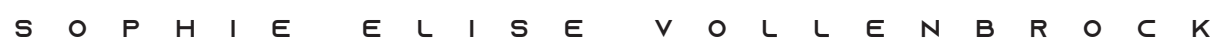




\section{COLOFON}

The work described in this thesis was performed at the Netherlands Cancer Institute - Antoni van Leeuwenhoek, Amsterdam, the Netherlands

() 2020, S.E. Vollenbrock

All rights reserved. No part of this thesis may be reproduced, stored or transmitted, in any form or by any means, without prior permission of the author.

Cover art: Sophie Vollenbrock

Lay-out: Sophie Vollenbrock

Printed by: Ipskamp Printing, Enschede, the Netherlands

ISBN: $\quad$ 978-94-6421-126-9

The Netherlands Cancer Institute, Maastricht University and the Nederlandse Vereniging voor Gastroenterologie (NVGE) kindly provided financial support for publication of this thesis. 


\section{MRI \\ FOR PERSONALISED TREATMENT \\ in \\ OESOPHAGEAL CANCER}

\section{Proefschrift}

ter verkrijging van de graad van doctor aan de Universiteit Maastricht

op gezag van de rector magnificus, prof. dr. Rianne M. Letschert, volgens het besluit van het College van Decanen, in het openbaar te verdedigen op woensdag 9 december 2020 om 12.00 uur

$$
\text { door }
$$

Sophie Elise Vollenbrock

geboren op 9 september 1991

te Maarssen 
Promotor:

Prof. dr. R.G.H. Beets-Tan

Copromotoren:

Dr. A. Bartels-Rutten, Antoni van Leeuwenhoek

Dr. F.E.M. Voncken, Antoni van Leeuwenhoek

Beoordelingscommissie:

Prof. dr. A. Masclee (voorzitter)

Prof. dr. N.D. Bouvy

Prof. dr. K. Haustermans, UZ Leuven

Prof. dr. L.P.S. Stassen

Prof. dr. J. Stoker, Amsterdam UMC 


\section{TABLE OF CONTENTS}

CHAPTER 1 General Introduction

CHAPTER 2 Diagnostic performance of MRI for assessment of response to neoadjuvant chemoradiotherapy in oesophageal cancer

CHAPTER 3 Diffusion-weighted MRI with ADC mapping for response prediction and assessment of oesophageal cancer: A systematic review

CHAPTER 4 Clinical response assessment on DW-MRI compared with FDG-PET/ $\mathrm{CT}$ after neoadjuvant chemoradiotherapy in patients with oesophageal cancer

CHAPTER 5 Added value of MRI to endoscopic and endosonographic response 79 assessment after neoadjuvant chemoradiotherapy in oesophageal cancer

CHAPTER 6 Gross tumor delineation in esophageal cancer on MRI compared with 99 18F-FDG-PET/CT

CHAPTER 7 General Discussion

APPENDICES Summary

Samenvatting

Valorisation

131

List of Publications

135

List of Contributing Authors

139

Dankwoord

145

Curriculum Vitae 


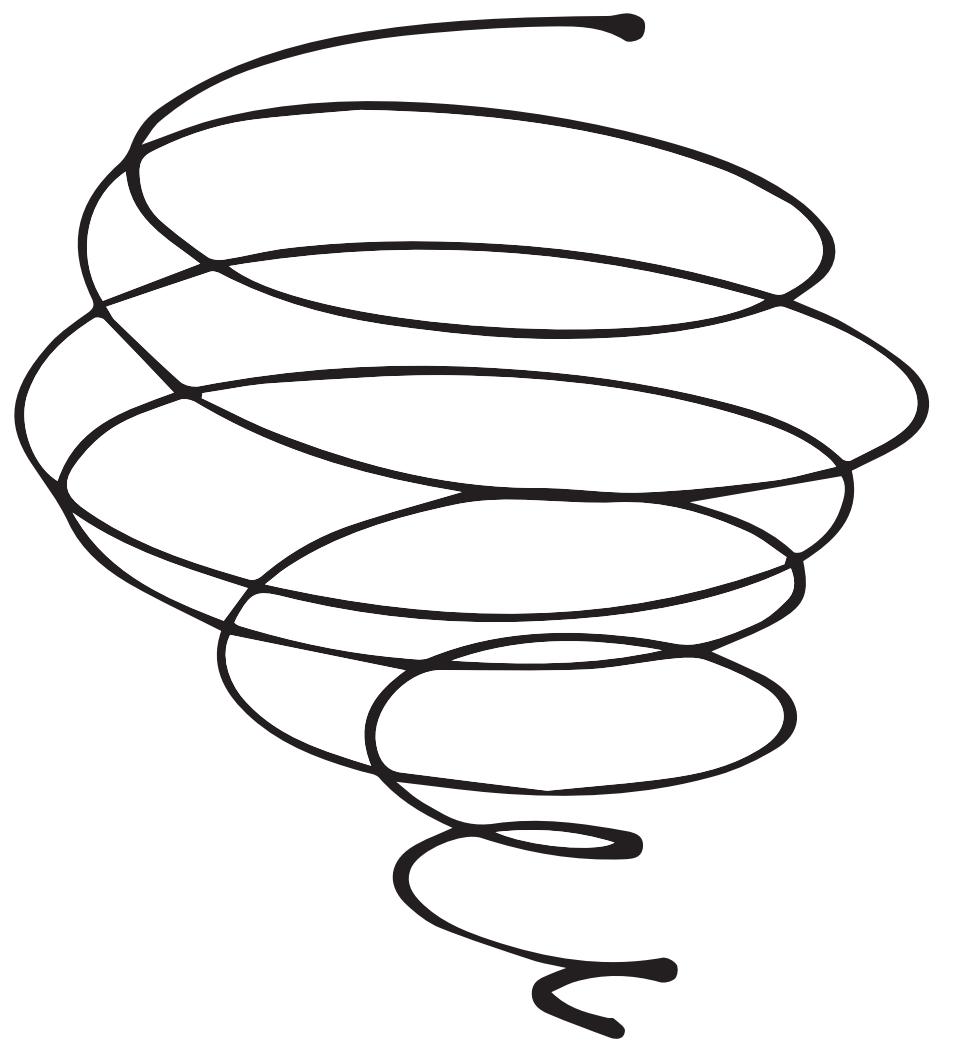




\section{CHAPTER 1}

General Introduction 
Oesophageal cancer is diagnosed in more than 500,000 patients per year worldwide and with a 5 -year survival of $15-25 \%$, it is the sixth leading cause of cancer-related mortality'. There are two major histologic subtypes; squamous cell - and adenocarcinoma. Squamous cell carcinomas are predominantly located in the proximal oesophagus and most common in Asia, Africa and Southern America'. Risk factors are alcohol consumption and tobacco smoking ${ }^{2}$. In Europe, North America and Oceania adenocarcinoma of the distal oesophagus predominates ${ }^{3,4}$. Important risk factors for adenocarcinomas include obesity and gastro-oesophageal reflux disease ${ }^{5}$ and in many high-income countries adenocarcinoma rates has already surpassed or are predicted to surpass the incidence of squamous cell carcinomas ${ }^{6}$. Overall, oesophageal cancer rates are substantially higher in men than in women'.

Treatment historically consisted of surgery alone with a median overall survival of 2 years ${ }^{7}$. With the introduction of preoperative treatment strategies (e.g. chemotherapy, radiotherapy) the oncological outcomes improved 8 . In the Netherlands, neoadjuvant chemoradiotherapy (nCRT) followed by surgery is currently standard of care due to the higher survival rates and improved locoregional control as compared to surgery alone ${ }^{7,9}$.

The response to neoadjuvant treatment is currently determined at histopathology of the resection specimen after surgery ${ }^{10}$. It has been shown that with current chemoradiotherapy schemes, locally advanced tumours show a pathological complete response in $29 \%$ of the patients ${ }^{7}$. This has raised the question whether in this group of complete responders major resection surgery with high morbidity and risk for mortality is still justified ${ }^{11-13}$. An active surveillance strategy is not (yet) adopted as an alternative treatment but some retrospective cohort studies suggest noninferior oncological outcomes for active surveillance patients compared to standard surgical resection ${ }^{14-16}$. However, in patients staged as CCR treated with esophagectomy, in 18-55 per cent residual tumour was found in the resected tumour bed ${ }^{14-17}$.

Hence the current debate is whether organ preservation could be a safe alternative to surgery in patients with a clinical complete response to chemoradiotherapy. Critical is the selection and surveillance of the eligible patients. Three important questions need to be addressed: 1 . Is there any residual disease in the irradiated tumour bed? 2. Are the lymph nodes sterilised? 3. Can we detect tumour regrowth at a curable stage during the active surveillance?

Current literature reports the accuracy of response evaluation after chemoradiotherapy using endoscopy ${ }^{18,19}$. Endoscopy provides information of only the luminal side of the tumour bed while residual disease is located beneath the mucosal wall layer in $28 \%$ of patients ${ }^{20,21}$. Therefore, endoscopic biopsies have a high number of false-negatives ${ }^{22}$. Endoscopic ultrasound (EUS) can more accurately visualise the deeper layers of the wall and differentiate visible lymph nodes. EUS 
identifies $52 \%$ of complete responders based on a cut-off of residual wall thickness of $4.5 \mathrm{~mm}^{23}$. EUS combined with fine needle aspiration (EUS-FNA) detected $50 \%$ of patients with residual nodal disease after chemoradiotherapy ${ }^{24}$. Therefore, clinical assessment by endoscopy and EUS after chemoradiotherapy are insufficient to reliably select the patients for non-operative management. These shortcomings in clinical assessment warrant further investigation of imaging techniques to complement endoscopy and EUS.

Currently, FDG-PET/CT is part of the standard work up to rule out distant metastases and select patients eligible for preoperative chemoradiotherapy. Several studies support the use of FDG-PET/ $\mathrm{CT}$ to detect intermittent distant metastases after neoadjuvant chemoradiotherapy ${ }^{25-30}$. The role of FDG-PET/CT for the assessment of local tumour response after neoadjuvant chemoradiotherapy has so far been limited because the differentiation between residual tumour, fibrosis and radiation oesophagitis remains difficult $27,31-34$.

Advances in MRI technology have led to high resolution imaging of the oesophagus and proper visualisation of the oesophageal wall ${ }^{35-39}$. MRI also provides the opportunity to perform functional imaging such as diffusion-weighted MRI (DW-MRI). DW-MRI is used to determine the water diffusivity in tissue ${ }^{40,41}$. An increase of the cell density, secondary to cell proliferation in tumours, results in a high signal on diffusion-weighted images due to a decrease in diffusivity of extracellular water. From the DW-MRI apparent diffusion coefficient (ADC) maps can be generated, representing a quantitative measure of water diffusivity in tissue ${ }^{41}$. While malignancies with a high cellularity cause diffusion restriction, fibrosis does not. In rectal cancer active surveillance strategies, DW-MRI has proven its additional value to clinical assessment (digital rectal examination and endoscopy) of response to chemoradiotherapy ${ }^{42,43}$. MRI is also increasingly explored in studies focusing on response prediction of oesophageal cancer ${ }^{44}$.

We hypothesise that DW-MRI can improve the performance of clinical assessment of response to preoperative chemoradiotherapy and identifies patients with complete response who would be eligible for active surveillance.

The aim of this thesis is to explore the role of MRI for personalised treatment in locally advanced oesophageal cancer.

The main study questions are:

- What is the diagnostic accuracy of clinical response evaluation on DW-MRI after chemoradiotherapy?

- What is the role for clinical response evaluation on DW-MRI in a multimodality diagnostic approach?

- Can DW-MRI be used for radiotherapy treatment planning? 
Outline of this thesis

In Chapter 2 the visual response assessment on DW-MRI by three oncological specialised radiologists was explored. Chapter 3 evaluates the potential predictive value of quantitative diffusion-weighted MRI by performing a systematic review. Chapter 4 compares clinical response evaluation after nCRT on DW-MRI to FDG-PET/CT. Subsequently, Chapter 5 investigates the additional value of DW-MRI assessment to endoscopy and EUS. Lastly, Chapter 6 compares delineation variation on DW-MRI to currently used FDG-PET/CT to investigate the role of DW-MRI for radiotherapy treatment planning purposes. 


\section{REFERENCES}

1. Bray F, Ferlay J, Soerjomataram I, Siegel RL, Torre LA, Jemal A. Global cancer statistics 2018: GLOBOCAN estimates of incidence and mortality worldwide for 36 cancers in 185 countries. CA: a cancer journal for clinicians 2018;68:394-424.

2. Arnold M, Soeriomataram I, Ferlay J, Forman D. Global incidence of oesophageal cancer by histological subtype in 2012. Gut 2015;64:3817.

3. Dikken JL, Lemmens VE, Wouters MW, et al. Increased incidence and survival for oesophageal cancer but not for gastric cardia cancer in the Netherlands. Eur J Cancer 2012;48:1624-32.

4. Pennathur A, Gibson MK, Jobe BA, Luketich JD. Oesophageal carcinoma. Lancet 2013;381:40012.

5. Uhlenhopp DJ, Then EO, Sunkara T, Gaduputi V. Epidemiology of esophageal cancer: update in global trends, etiology and risk factors. Clin J Gastroenterol. 2020.

6. Arnold M, Laversanne $M$, Brown LM, Devesa SS, Bray F. Predicting the Future Burden of Esophageal Cancer by Histological Subtype: International Trends in Incidence up to 2030. Am J Gastroenterol 2017;112:1247-55.

7. van Hagen $P$, Hulshof MC, van Lanschot JJ, et al. Preoperative chemoradiotherapy for esophageal or junctional cancer. N Engl J Med 2012;366:2074-84.

8. Markar SR, Noordman BJ, Mackenzie H, et al. Multimodality treatment for esophageal adenocarcinoma: multi-center propensity-score matched study. Ann Oncol 2017;28:519-27.

9. Shapiro J, van Lanschot JJB, Hulshof $M$, et al. Neoadjuvant chemoradiotherapy plus surgery versus surgery alone for oesophageal or junctional cancer (CROSS): long-term results of a randomised controlled trial. Lancet Oncol 2015;16:1090-8.

10. Mandard AM, Dalibard F, Mandard JC, et al. Pathologic assessment of tumor regression after preoperative chemoradiotherapy of esophageal carcinoma. Clinicopathologic correlations. Cancer 1994;73:2680-6.

11. Busweiler LA, Wijnhoven BP, van Berge
Henegouwen $\mathrm{Ml}$, et al. Early outcomes from the Dutch Upper Gastrointestinal Cancer Audit. Br J Surg 2016;103:1855-63.

12. Schandl A, Lagergren J, Johar A, Lagergren P. Health-related quality of life 10 years after oesophageal cancer surgery. Eur J Cancer 2016;69:43-50.

13. Triantafyllou T, Wijnhoven B. Multidisciplinary treatment of esophageal cancer: The role of active surveillance after neoadjuvant chemoradiation. Ann Gastroenterol Surg 2020;4:352-9.

14. Castoro C, Scarpa M, Cagol M, et al. Complete clinical response after neoadjuvant chemoradiotherapy for squamous cell cancer of the thoracic oesophagus: is surgery always necessary? J Gastrointest Surg 2013;17:137581.

15. Furlong $H$, Bass $G$, Breathnach $O, O^{\prime}$ Neill $B$, Leen E, Walsh TN. Targeting therapy for esophageal cancer in patients aged 70 and over. J Geriatr Oncol 2013;4:107-13.

16. Ohkura Y, Shindoh J, Ueno M, lizuka T, Udagawa $\mathrm{H}$. Comparison of Outcome of Esophagectomy Versus Nonsurgical Treatment for Resectable Esophageal Cancer with Clinical Complete Response to Neoadjuvant Therapy. Ann Surg Oncol 2018;25:2428-33.

17. Noordman BJ, Spaander MCW, Valkema R, et al. Detection of residual disease after neoadjuvant chemoradiotherapy for oesophageal cancer (preSANO): a prospective multicentre, diagnostic cohort study. Lancet Oncol 2018;19:965-74.

18. Noordman BJ, Shapiro J, Spaander MCW, et al. Accuracy of Detecting Residual Disease After Cross Neoadjuvant Chemoradiotherapy for Esophageal Cancer (preSANO Trial): Rationale and Protocol. JMIR Research Protocols 2015;4:e79.

19. van Rossum PSN, Goense L, Meziani J, et al. Endoscopic biopsy and EUS for the detection of pathologic complete response after neoadjuvant chemoradiotherapy in esophageal cancer: a systematic review and meta-analysis. Gastrointest Endosc 2015;83:866-79.

20. Shapiro J, ten Kate FJ, van Hagen P, Biermann $\mathrm{K}$, Wijnhoven BP, van Lanschot JJ. Residual esophageal cancer after neoadjuvant 
chemoradiotherapy frequently involves the mucosa and submucosa. Ann Surg 2013;258:678-88; discussion 88-89.

21. Chao YK, Chang Y, Yeh CJ, Chang HK, Tseng CK, Chuang WY. Characterization of residual tumours at the primary site in patients with a near pathological complete response after neoadjuvant chemoradiotherapy for oesophageal cancer. $\mathrm{Br} \mathrm{J}$ Surg 2016;103:1874-9.

22. Chao YK, Yeh CJ, Lee MH, et al. Factors associated with false-negative endoscopic biopsy results after neoadjuvant chemoradiotherapy in patients with esophageal squamous cell carcinoma. Medicine (Baltimore) 2015;94:e588.

23. van der Bogt RD, Noordman BJ, Krishnadath KK, et al. Endoscopic ultrasound measurements for detection of residual disease after neoadjuvant chemoradiotherapy for esophageal cancer. Endoscopy 2019;51:326-32.

24. van der Bogt RD, van der Wilk BJ, Poley JW, et al. Endoscopic ultrasound and fine-needle aspiration for the detection of residual nodal disease after neoadjuvant chemoradiotherapy for esophageal cancer. Endoscopy 2020;52:186-92.

25. Bruzzi JF, Swisher SG, Truong MT, et al. Detection of interval distant metastases: clinical utility of integrated CT-PET imaging in patients with esophageal carcinoma after neoadjuvant therapy. Cancer 2007;109:125-34.

26. Blom RL, Schreurs WM, Belgers HJ, Oostenbrug $\mathrm{LE}$, Vliegen RF, Sosef MN. The value of postneoadjuvant therapy PET-CT in the detection of interval metastases in esophageal carcinoma. Eur J Surg Oncol. 2011 ; 37:774-8.

27. Stiekema J, Vermeulen D, Vegt $E$, et al. Detecting interval metastases and response assessment using 18F-FDG PET/CT after neoadjuvant chemoradiotherapy for esophageal cancer. Clin Nucl Med 2014;39:862-7.

28. Anderegg MC, de Groof EJ, Gisbertz SS, et al. 18F-FDG PET-CT after Neoadjuvant Chemoradiotherapy in Esophageal Cancer Patients to Optimize Surgical Decision Making. PLoS One 2015;10:e133690.

29. Goense L, Ruurda JP, Carter BW, et al. Prediction and diagnosis of interval metastasis after neoadjuvant chemoradiotherapy for oesophageal cancer using (18)F-FDG PET/CT. Eur J Nucl Med Mol Imaging 2018;45:1742-51.
30. Kroese TE, Goense L, van Hillegersberg R, et al. Detection of distant interval metastases after neoadjuvant therapy for esophageal cancer with 18F-FDG PET(/CT): a systematic review and meta-analysis. Dis Esophagus 2018;31.

31. Cheedella NK, Suzuki A, Xiao L, et al. Association between clinical complete response and pathological complete response after preoperative chemoradiation in patients with gastroesophageal cancer: analysis in a large cohort. Ann Oncol 2013;24:1262-6.

32. van Rossum PS, Fried DV, Zhang $L$, et al. The Incremental Value of Subjective and Quantitative Assessment of 18F-FDG PET for the Prediction of Pathologic Complete Response to Preoperative Chemoradiotherapy in Esophageal Cancer. J Nucl Med 2016;57:691-700.

33. Arnett ALH, Merrell KW, Macintosh EM, et al. Utility of (18)F-FDG PET for Predicting Histopathologic Response in Esophageal Carcinoma following Chemoradiation. J Thorac Oncol 2017;12:121-8.

34. Lee J, Choi JY, Lim SW, et al. Utility of positron emission-computed tomography for predicting pathological response in resectable oesophageal squamous cell carcinoma after neoadjuvant chemoradiation. Eur J Cardiothorac Surg 2020;58:1019-26.

35. Ehman RL, Felmlee JP. Adaptive technique for high-definition MR imaging of moving structures. Radiology 1989;173:255-63.

36. Lever FM, Lips IM, Crijns SP, et al. Quantification of esophageal tumor motion on cine-magnetic resonance imaging. Int J Radiat Oncol Biol Phys 2014;88:419-24.

37. Riddell AM, Davies DC, Allum WH, Wotherspoon $A C$, Richardson C, Brown G. High-resolution MRI in evaluation of the surgical anatomy of the esophagus and posterior mediastinum. AJR Am J Roentgenol 2007;188:W37-43.

38. Riddell AM, Richardson C, Scurr E, Brown G. The development and optimization of high spatial resolution MRI for imaging the oesophagus using an external surface coil. $\mathrm{Br} J$ Radiol 2006;79:873-9.

39. Qu J, Wang Z, Qin J, et al. MRI features in differentiating mucosal high-grade neoplasia from early invasive squamous cell cancer of the esophagus. Eur Radiol 2020;30:3455-61. 
40. Le Bihan D, Poupon C, Amadon A, Lethimonnier F. Artifacts and piffalls in diffusion MRI. J Magn Reson Imaging 2006;24:478-88.

41. Koh DM, Collins DJ. Diffusion-weighted MRI in the body: applications and challenges in oncology. AJR Am J Roentgenol 2007;1 188:1622-35.

42. Maas M, Lambregts DM, Nelemans PJ, et al. Assessment of Clinical Complete Response After Chemoradiation for Rectal Cancer with Digital Rectal Examination, Endoscopy, and MRI: Selection for Organ-Saving Treatment. Ann Surg Oncol 2015;22:3873-80.

43. van der Valk MJM, Hilling DE, Bastiaannet $E$, et al. Long-term outcomes of clinical complete responders after neoadjuvant treatment for rectal cancer in the International Watch \& Wait Database (IWWD): an international multicentre registry study. Lancet 2018;391:2537-45.

44. Maffazzioli L, Zilio MB, Klamt AL, et al. ADC as a predictor of pathologic response to neoadjuvant therapy in esophageal cancer: a systematic review and meta-analysis. Eur Radiol 2020;30:3934-42. 


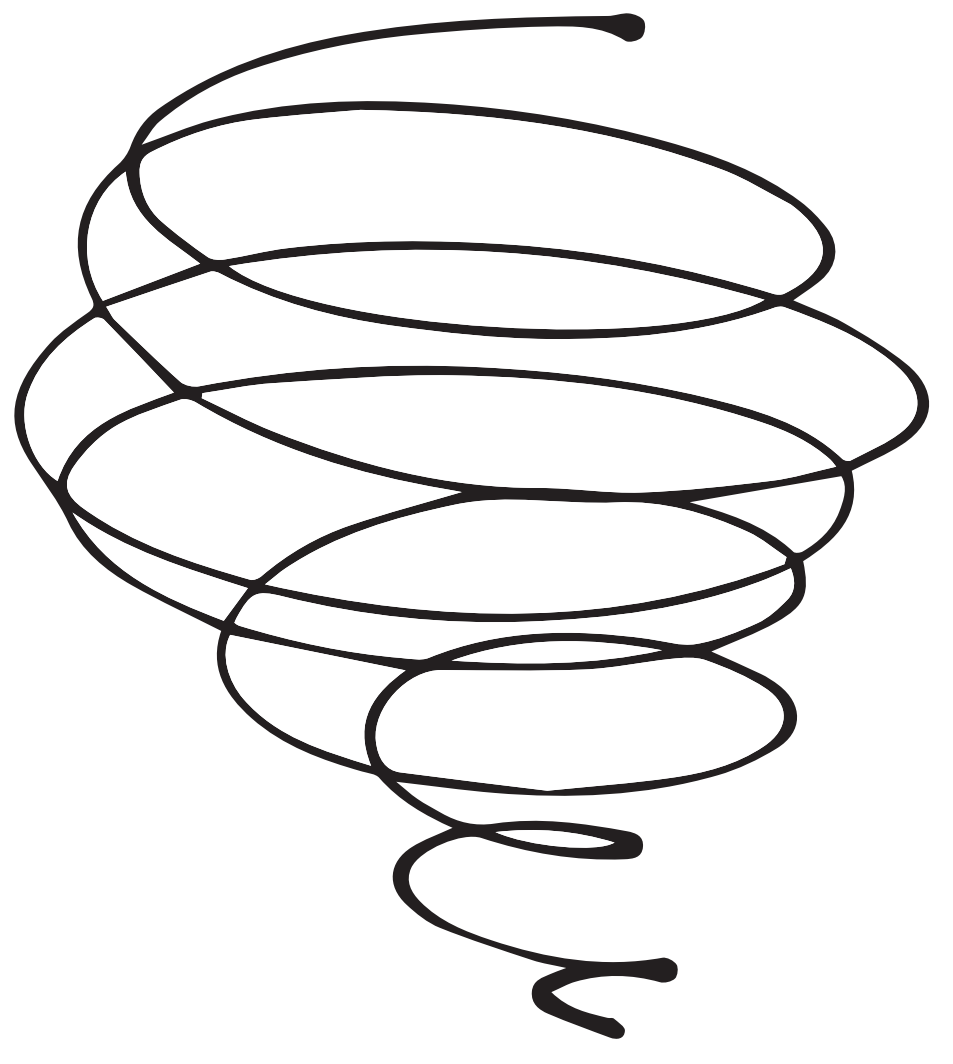




\title{
CHAPTER 2
}

\section{Diagnostic performance of MRI for assessment of response to neoadjuvant chemoradiotherapy in oesophageal
cancer chemoradiotherapy in oesophageal
cancer}

British Journal of Surgery; Feb 2019 (Volume 106, Issue 5, p596-605)

\author{
Sophie E. Vollenbrock* \\ Francine E.M. Voncken* \\ Jolanda M. van Dieren \\ Doenja M.J. Lambregts \\ Monique Maas \\ Gert J. Meijer \\ Lucas Goense \\ Stella Mook \\ Koen J. Hartemink \\ Petur Snaebjornsson \\ Leon C. ter Beek \\ Marcel Verheij \\ Berthe M.P. Aleman \\ Regina G.H. Beets-Tan \\ Annemarieke Bartels-Rutten
}

*Contributed equally 


\begin{abstract}
Background

Patients with a pathological complete response (pCR) after neoadjuvant chemoradiotherapy (nCRT) for oesophageal cancer may benefit from non-surgical management. The aim of this study was to determine the diagnostic performance of visual response assessment of the primary tumour after nCRT on T2-weighted (T2W) and diffusion-weighted (DW) MRI.
\end{abstract}

\title{
Methods
}

Patients with locally advanced oesophageal cancer who underwent T2W- and DW-MRI (1.5T) before and after nCRT in two hospitals, between July 2013 and September 2017, were included in this prospective study. Three radiologists evaluated $\mathrm{T} 2 \mathrm{~W}$ images retrospectively using a five-point score for the assessment of residual tumour in a blinded manner and immediately rescored after adding DW-MRI. Histopathology of the resection specimen was used as the reference standard; ypTO represented a pCR. Sensitivity, specificity, area under the receiver operating characteristic (ROC) curve (AUC) and interobserver agreement were calculated.

\section{Results}

Twelve of 51 patients (24 per cent) had a pCR. The sensitivity and specificity of T2W-MRI for detection of residual tumour ranged from 90 to 100 and 8 to 25 per cent respectively. Respective values for T2W+DW-MRI were 90-97 and 42-50 per cent. AUCs for the three readers were 0.65, 0.66 and 0.68 on T2W-MRI, and $0.71,0.70$ and 0.70 on T2W+DW-MRI $(P=0.441, P=0.611$ and $P=0.828$ for readers 1,2 and 3 respectively). The $\kappa$ value for interobserver agreement improved from $0.24-0.55$ on T2W-MRI to $0.55-0.71$ with DW-MRI.

\section{Conclusion}

Preoperative assessment of residual tumour on MRI after $n C R T$ for oesophageal cancer is feasible with high sensitivity, reflecting a low chance of missing residual tumour. However, the specificity was low; this results in overstaging of complete responders as having residual tumour and, consequently, overtreatment. 


\section{INTRODUCTION}

A standard therapy with curative intent for patients with locally advanced oesophageal cancer consists of neoadjuvant chemoradiotherapy (nCRT) followed by surgery. nCRT improves survival compared with surgery alone (5-year survival rate 47 versus 33 per cent respectively)'. In 25-30 per cent of patients with oesophageal cancer, the resection specimen shows no residual tumour cells (ypT0) after $\mathrm{nCRT}^{2,3}$, also known as a pathological complete response (pCR). Patients with a pCR have an excellent prognosis, with a 5-year recurrence-free survival rate of 62 per cent ${ }^{4,5}$, which is better than that of patients with vital tumour cells in the resection specimen (no pCR). The high postoperative morbidity and mortality rates after oesophagectomy raise the question of whether non-surgical management (watch and wait) is a safe alternative treatment option in patients with oesophageal cancer who have a clinical complete response (cCR) ${ }^{6}$.

To implement non-surgical treatment for advanced oesophageal cancer it is critical to accurately identify PCR. CT and fluorodeoxyglucose (FDG) PET/CT are both inaccurate in discriminating residual disease from $\mathrm{pCR}$ owing to the presence of wall thickening and/or radiation oesophagitis ${ }^{7-13}$. The same is true for endoscopic ultrasonography (EUS), which has an accuracy of only 36 per cent for ypT determination ${ }^{14,15}$. Endoscopic response evaluation after $\mathrm{nCRT}$ is hampered by the fact that it provides information only on the luminal side of the oesophagus ${ }^{16}$, whereas residual cancer cells are located beneath the mucosal layer in a subset of patients ${ }^{17,18}$. Even deeper bite-on-bite biopsies combined with EUS and fine-needle aspiration of suspicious lymph nodes yielded a negative predictive value (NPV) of only 45 per cent for detecting tumours with a tumour regression grade (TRG) of 2 or higher ${ }^{11}$. Previous retrospective cohort studies ${ }^{19-22}$ reported on patients with a cCR after neoadjuvant treatment who did not have surgery but underwent serial response assessments including endoscopy, EUS, CT and/or FDG-PET/CT. Among those assessed as having a cCR who did undergo oesophagectomy, residual tumour was found in 28-33 per cent of patients ${ }^{19-21}$. In a propensity-based matching study22, after a median follow-up of 51.1 months, local recurrence had developed more frequently among patients with a $\mathrm{CCR}$ who underwent a watch-and-wait approach than in those who had surgical treatment.

The limitations of the current response assessment tools warrant investigation of other imaging techniques. In rectal cancer, MRI can aid in the diagnosis of a cCR after $\mathrm{nCRT}^{23,24}$. Although MRI of the oesophagus is technically more challenging, owing to oesophageal motility and motion of the surrounding heart and diaphragm, advances in technology now enable the acquisition of highresolution magnetic resonance (MR) images. Previous studies ${ }^{25-29}$ on response prediction with MRI in oesophageal cancer focused on quantitative diffusion-weighted (DW) MRI parameters. However, visual response assessment on T2-weighted (T2W) and functional DW-MRI in rectal cancer yielded higher sensitivity for detecting residual tumour compared with quantitative assessment ${ }^{30}$. The performance of visual response assessment on MRI in oesophageal cancer is as yet unknown. The aim of this study was to determine the diagnostic performance of visual response assessment of the 
primary oesophageal tumour after nCRT on T2W-MRI and functional DW-MRI.

\section{METHODS}

Patients diagnosed with locally advanced oesophageal cancer were enrolled prospectively and data were analysed retrospectively. The study was approved by the local medical ethics committees and registered at ClinicalTrials.gov (NCT02139488 and NCT02125448). Written informed consent to participate was obtained from the patients. Patients underwent MRI before and after nCRT between July 2013 and September 2017. Inclusion criteria were: biopsy-proven locally advanced, nonmetastatic oesophageal cancer; 5 weeks of nCRT (total of 41.4 Gy in 23 fractions, with weekly administration of carboplatin and paclitaxel) followed by oesophagectomy; and maximum of 21 days between preoperative MRI and surgery. Patients were excluded if MRI quality was judged insufficient by at least two radiologists. Causes of insufficient image quality were severe motion artefacts leading to blurred T2W images, and lack of, or only slight, diffusion restriction in the spleen as a surrogate marker of inadequate DWimages.

\section{Image acquisition}

Imaging was performed on a 1.5-T MRI scanner (Achieva or Ingenia; Koninklijke Philips, Best, the Netherlands), using Torso-XL (16 channel) or anterior/posterior (28 channel) receiver coils respectively (supplied by Koninklijke Philips). The MRI protocol consisted of T2W multislice turbo spin-echo sequences in transverse (slice thickness $4 \mathrm{~mm}$ ) and sagittal (slice thickness $3 \mathrm{~mm}$ ) planes. A respiratory navigator was positioned on the diaphragm, and to reduce motion artefacts, images were acquired only during expiration ${ }^{31}$. A DW echo-planar imaging sequence was acquired in the transverse direction with $b=0, b=200$ and $b=800 \mathrm{~s} / \mathrm{mm}^{2}$, and a slice thickness of $4 \mathrm{~mm}$. The transverse T2W and DW sequences were angled in identical planes. Detailed MRI sequence parameters are provided in Table S1 (supporting information).

Image evaluation

Images were analysed independently by three expert radiologists, who were blinded to tumour characteristics (location, histology, TNM stage ${ }^{32}$ ) and clinical outcomes. The radiologists first judged images of the primary tumour area acquired by T2W-MRI and DW-MRI before $\mathrm{nCRT}$. They then scored the images obtained by T2W-MRI after nCRT for the likelihood of residual tumour. Finally, still in the same reading session, DW-MR images of $b=800 \mathrm{~s} / \mathrm{mm}^{2}$ were added and the scoring was repeated. A five-point confidence level score (CLS) was used, derived from previous studies in rectal cancer (CLS1, definitely complete response; 2 , probably complete response; 3 , inconclusive; 4 , probably residual tumour; 5 , definitely residual tumour $)^{23}$. 
MRI criteria

A complete response of the primary tumour on T2W-MRI was defined by a normalized oesophageal wall or only a thin hypointense signal (indicating fibrosis) without distortion of the wall; on DW-MRI, a complete response of the primary tumour was defined by the absence of high signal on images of $b=800 \mathrm{~s} / \mathrm{mm}^{2}$ in the irradiated tumour bed. On T2W-MRI, a residual mass with persistent isointense signal or the presence of mixed hyperintense and hypointense signals within the tumour bed were considered signs of residual tumour; on DW-MRI, the presence of high signal within the tumour bed indicated residual tumour. These criteria are illustrated in Figs 1 and 2.

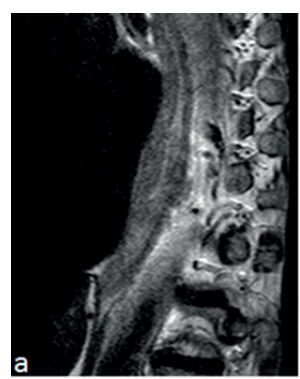

a. $\mathrm{T} 2 \mathrm{~W}$ sagittal image before nCRT

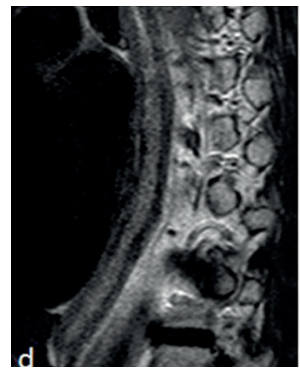

d. T2W sagittal image after nCRT

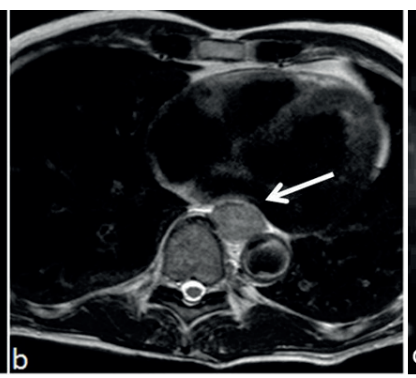

b. T2W transverse image before nCRT

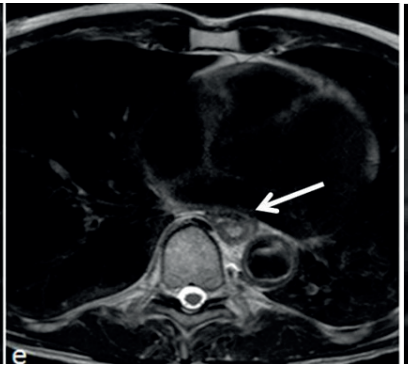

e. T2W transverse image after nCRT

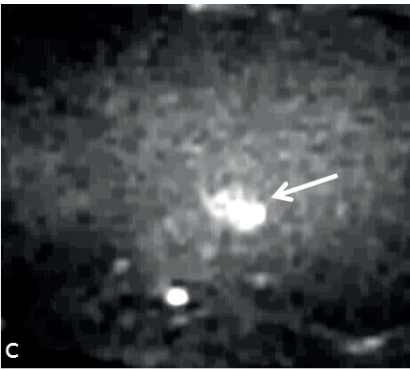

c. DW image before $\mathrm{nCRT}$

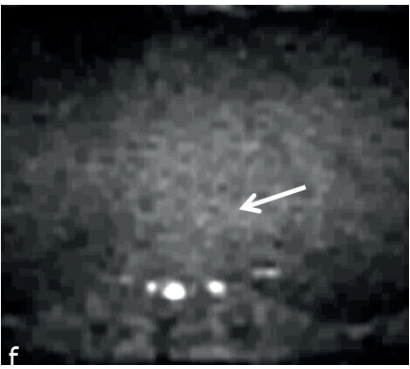

f. DW image after nCRT

Figure 1. MRI of a patient with locally advanced oesophageal cancer that showed a pathological complete response to neoadjuvant chemoradiotherapy Images from a 55-year-old man with a cT3NO lower oesophageal squamous cell carcinoma, and a complete pathological response after neoadjuvant chemoradiotherapy (nCRT) and oesophagectomy (tumour regression grade 1, ypTO NO). a-c: T2-weighted (T2W) sagittal (a) and transverse (b) images before chemoradiotherapy show a hyperintense oesophageal wall, accompanied by a hyperintense signal on diffusion-weighted (DW) imaging (c). $d-f$ : T2W sagittal (d) and transverse (e) images after nCRT show a hypointense oesophageal wall, indicating fibrosis; no high signal remained on the corresponding DW image (f). Arrows mark (initial) tumour location. 


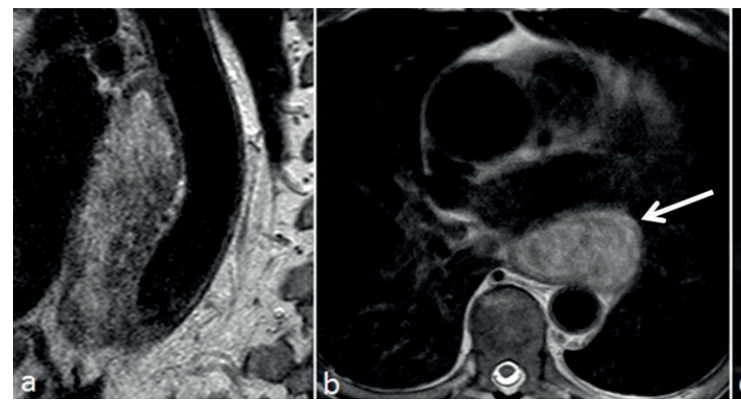

a. T2W sagittal image before nCRT

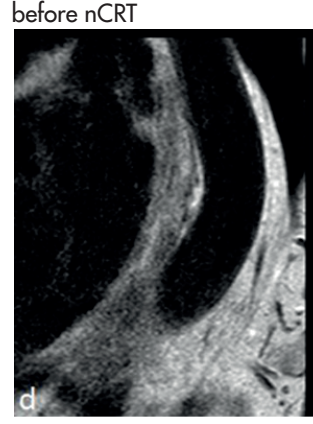

d. T2W sagittal image after nCRT b. T2W transverse image before nCRT

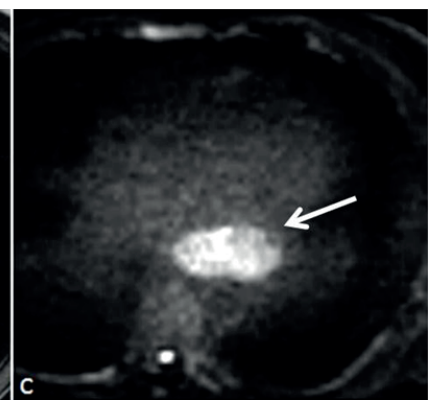

c. DW image before nCRT

f. DW image after nCRT

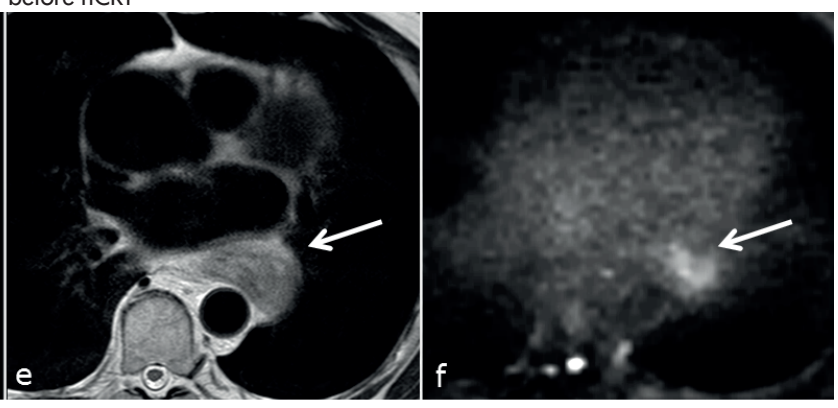

e. T2W transverse image after nCRT

Figure 2. MRI of a patient with locally advanced oesophageal cancer that showed pathological residual tumour after chemoradiotherapy and surgery Images from a 78-year-old man with a cT2 NO lower oesophageal adenocarcinoma, who had residual tumour after neoadjuvant chemoradiotherapy (nCRT) and oesophagectomy (tumour regression grade 5, ypT2 N0). T2-weighted (T2W) sagittal (a,d) and transverse (b,e) images before $(a, b)$ and after $(d, e) n C R T$ both show a hyperintense oesophageal wall. The corresponding $b=800$ diffusion-weighted (DW) images before (c) and after (f) nCRT demonstrate a clear hyperintense signal, highly suspicious for tumour. Arrows mark tumour location.

\section{Reference standard}

Histopathological examination of the resection specimen was performed by dedicated gastrointestinal pathologists at the two centres. The resection specimen was evaluated in accordance with the seventh edition of the UICC protocol for ypTNM classification ${ }^{32}$. The TRG of the resected primary tumour was assessed according to Mandard and colleagues ${ }^{3}$. The tumour bed was embedded completely for histopathological analysis. A pCR was defined as ypTO (TRG 1) and residual tumour as ypT1-4 (TRG 2-5). 


\section{RESULTS}

Six of 57 patients were excluded: four had insufficient MRI quality, one patient had distant metastases after completion of $\mathrm{nCRT}$ and therefore did not undergo surgical resection, and one patient was deemed to have unresectable disease at surgical exploration. Therefore, 51 patients were evaluated (Fig. 3). Of the 51 included patients, 42 (82 per cent) were diagnosed with adenocarcinoma and nine (18 per cent) with squamous cell carcinoma. Table 1 shows baseline patient and tumour characteristics at both institutes that participated in the study. Transhiatal oesophagectomy was performed in 24 patients (47 per cent) and transthoracic oesophagectomy in 27 (53 per cent), followed by gastric conduit reconstruction with cervical anastomosis in all patients. The median interval between the last radiation fraction and oesophagectomy was 59 (range 24-75) days. It was 47 (17-65) days between the last radiation fraction and MRI, and 11 (4-21) days from post-nCRT MRI until oesophagectomy. On histopathological assessment, a pCR of the primary tumour (ypTO, TRG 1) was found in 12 of 51 patients (24 per cent). In one of these patients, the disease was confirmed as ypTON1, which was regarded a complete response of the primary tumour area (урT0) in this study. The $\mathrm{pCR}$ rate was five of nine (56 per cent) for squamous cell carcinomas and seven of 42 (17 per cent) for adenocarcinomas. The remaining 39 patients had residual tumour, which was graded as TRG 2 in 17 of 51 patients (33 per cent), TRG 3 in 16 (31 per cent), TRG 4 in four (8 per cent) and TRG 5 in two patients ( 4 per cent).

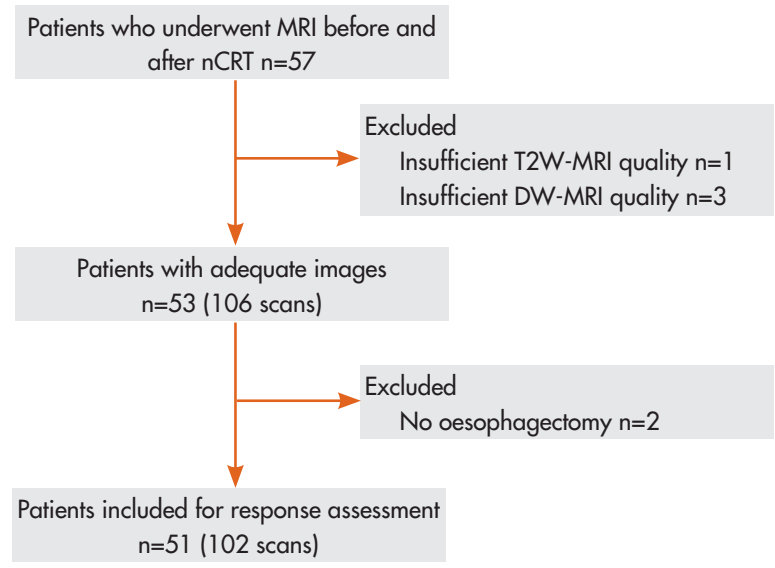

Figure 3. Study flow chart

nCRT, neoadjuvant chemoradiotherapy; T2W, T2-weighted; DW, diffusion-weighted. 
Table 1. Patient and tumour characteristics

\begin{tabular}{|c|c|c|c|}
\hline & Hospital 1 ( $n=32)$ & Hospital $2(n=19)$ & Total $(n=51)$ \\
\hline Age (years)* & $64(53-72)$ & $64(60-68)$ & $64(56-72)$ \\
\hline Sex ratio $(M: F)$ & $23: 9$ & $16: 3$ & $39: 12$ \\
\hline $\begin{array}{l}\text { Tumour location } \\
\text { Upper oesophageal } \\
\text { Middle oesophageal } \\
\text { Lower oesophageal } \\
\text { Gastro-oesophageal junction }\end{array}$ & $\begin{array}{c}0 \\
3 \\
15 \\
14 \\
\end{array}$ & $\begin{array}{c}1 \\
3 \\
11 \\
4 \\
\end{array}$ & $\begin{array}{c}1(2) \\
6(12) \\
26(51) \\
18(35) \\
\end{array}$ \\
\hline $\begin{array}{l}\text { Histological tumour type } \\
\text { Adenocarcinoma } \\
\text { Squamous cell carcinoma }\end{array}$ & $\begin{array}{c}28 \\
4 \\
\end{array}$ & $\begin{array}{c}14 \\
5 \\
\end{array}$ & $\begin{array}{l}42(82) \\
9(18)\end{array}$ \\
\hline $\begin{array}{l}\text { Grade of differentiation } \\
\text { Well differentiated } \\
\text { Moderately differentiated } \\
\text { Poorly differentiated } \\
\text { Undifferentiated } \\
\text { Unknown }\end{array}$ & $\begin{array}{c}2 \\
15 \\
14 \\
0 \\
1\end{array}$ & $\begin{array}{c}0 \\
10 \\
3 \\
2 \\
4\end{array}$ & $\begin{array}{l}2(4) \\
25(49) \\
17(33) \\
2(4) \\
5(10)\end{array}$ \\
\hline $\begin{array}{l}\text { Clinical T category } \dagger \\
\text { cT1 } \\
\text { cT2 } \\
\text { cT3 } \\
\text { cT4a }\end{array}$ & $\begin{array}{c}1 \\
8 \\
23 \\
0 \\
\end{array}$ & $\begin{array}{c}0 \\
4 \\
14 \\
1 \\
\end{array}$ & $\begin{array}{c}1(2) \\
12(24) \\
37(73) \\
1(2) \\
\end{array}$ \\
\hline $\begin{array}{l}\text { Clinical } \mathrm{N} \text { category } \dagger \\
\mathrm{cN} 0 \\
\mathrm{cN} 1 \\
\mathrm{cN} 2 \\
\mathrm{cN} 3\end{array}$ & $\begin{array}{l}15 \\
6 \\
9 \\
2 \\
\end{array}$ & $\begin{array}{l}4 \\
8 \\
7 \\
0\end{array}$ & $\begin{array}{c}19(37) \\
14(27) \\
16(31) \\
2(4) \\
\end{array}$ \\
\hline $\begin{array}{l}\text { Radicality of resection } \\
\text { RO } \\
\text { R1 }\end{array}$ & $\begin{array}{c}32 \\
0\end{array}$ & $\begin{array}{c}18 \\
1\end{array}$ & $\begin{array}{c}50(98) \\
1(2)\end{array}$ \\
\hline $\begin{array}{l}\text { Mandard grade } \\
\text { TRG } 1 \\
\text { TRG } 2 \\
\text { TRG } 3 \\
\text { TRG } 4 \\
\text { TRG } 5\end{array}$ & $\begin{array}{c}6 \\
10 \\
12 \\
3 \\
1\end{array}$ & $\begin{array}{l}6 \\
7 \\
4 \\
1 \\
1\end{array}$ & $\begin{array}{c}12(24) \\
17(33) \\
16(31) \\
4(8) \\
2(4)\end{array}$ \\
\hline
\end{tabular}

Values in parentheses are percentages unless indicated otherwise; ${ }^{*}$ values are median (i.q.r.).

† According to the seventh edition of the TNM classification ${ }^{32}$

Hospital 1, Netherlands Cancer Institute; Hospital 2, University Medical Center Utrecht. TRG, tumour regression grade.

Diagnostic performance

ROC curves for the assessment of residual tumour after nCRT are shown in Fig. 4. AUCs on T2WMRI were 0.65 for reader $1,0.66$ for reader 2 and 0.68 for reader 3 . After addition of DW-MRI, $A U C$ s were $0.71,0.70$ and 0.70 respectively $(P=0.441, P=0.611$ and $P=0.828)$. The sensitivity for detection of residual tumour ranged from 90 to 100 per cent on T2W-MRI alone, and from 90 to 97 per cent after addition of DW-MRI. Specificity ranged from 8 to 25 per cent on T2W-MRI alone, and from 42 to 50 per cent after addition of DW-MRI (Table 2). The numbers of MRI-positive and -negative tests per TRG stage for response assessment on T2W+DW-MRI are shown in Table 3. 
a. Radiologist 1

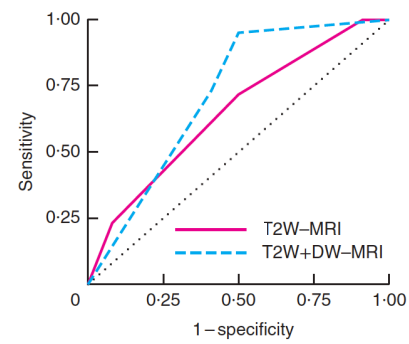

b. Radiologist 2

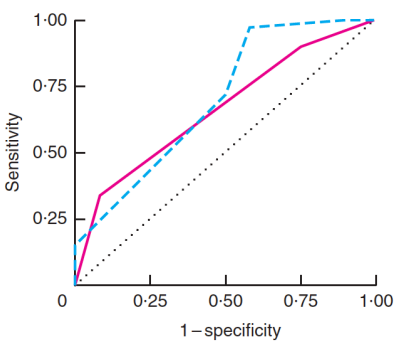

c. Radiologist 3

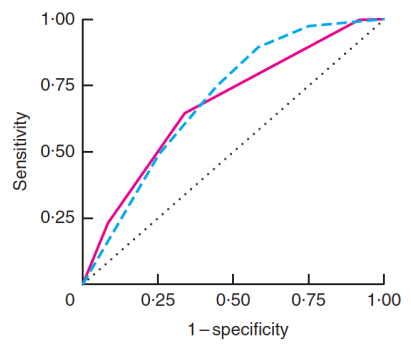

Figure 4. Receiver operating characteristic curves for assessment of residual tumour after neoadjuvant chemoradiotherapy using T2-weighted MRI and T2-weighted combined with diffusionweighted MRI.

a reader $1, b$ reader 2 and c reader 3. T2W, T2-weighted; DW, diffusion-weighted.

Comparison of areas under the curve for T2W-MRI versus T2W+DW-MRI:

a $\mathrm{P}=0.441, \mathrm{~b} \mathrm{P}=0.611, \mathrm{cP}=0.828$ (Delong tes ${ }^{33}$ ).

Table 2. Diagnostic performance for assessment of residual tumour

\begin{tabular}{|c|c|c|c|c|c|c|}
\hline & \multicolumn{3}{|c|}{ T2W-MRI } & \multicolumn{3}{|c|}{$\mathrm{T} 2 \mathrm{~W}+\mathrm{DW}-\mathrm{MRI}$} \\
\hline & Reader 1 & Reader 2 & Reader 3 & Reader 1 & Reader 2 & Reader 3 \\
\hline ty (\%) & $\begin{array}{c}100 \\
(89,100)\end{array}$ & $\begin{array}{c}90 \\
(75,97) \\
\end{array}$ & $\begin{array}{c}90 \\
(75,97) \\
\end{array}$ & $\begin{array}{c}95 \\
(81,99) \\
\end{array}$ & $\begin{array}{c}97 \\
(85,100) \\
\end{array}$ & $\begin{array}{c}90 \\
(75,97) \\
\end{array}$ \\
\hline Specificity (\%) & $\begin{array}{c}8 \\
(0,40)\end{array}$ & $\begin{array}{c}25 \\
(7,57)\end{array}$ & $\begin{array}{c}25 \\
(7,57)\end{array}$ & $\begin{array}{c}50 \\
(22,78) \\
\end{array}$ & $\begin{array}{c}42 \\
(17,71)\end{array}$ & $\begin{array}{c}42 \\
(17,71)\end{array}$ \\
\hline PPV (\%) & $\begin{array}{c}78 \\
(64,88)\end{array}$ & $\begin{array}{c}80 \\
(64,90)\end{array}$ & $\begin{array}{c}80 \\
(64,90)\end{array}$ & $\begin{array}{c}86 \\
(71,94)\end{array}$ & $\begin{array}{c}84 \\
(70,93)\end{array}$ & $\begin{array}{c}83 \\
(68,92)\end{array}$ \\
\hline NPV (\%) & $\begin{array}{c}100 \\
(6,100)\end{array}$ & $\begin{array}{c}43 \\
(12,80)\end{array}$ & $\begin{array}{c}43 \\
(12,80)\end{array}$ & $\begin{array}{c}75 \\
(36,96)\end{array}$ & $\begin{array}{c}83 \\
(37,99)\end{array}$ & $\begin{array}{c}56 \\
(23,85) \\
\end{array}$ \\
\hline True-positive & 39 & 35 & 35 & 37 & 38 & 35 \\
\hline False-positive & 11 & 9 & 9 & 6 & 7 & 7 \\
\hline True-negative & 1 & 3 & 3 & 6 & 5 & 5 \\
\hline False-negative & 0 & 4 & 4 & 2 & 1 & 4 \\
\hline Accuracy (\%) & 78 & 75 & 75 & 84 & 84 & 78 \\
\hline AUC* & $\begin{array}{c}0.65 \\
(0.47,0.83)\end{array}$ & $\begin{array}{c}0.66 \\
(0.49,0.83)\end{array}$ & $\begin{array}{c}0.68 \\
(0.51,0.86)\end{array}$ & $\begin{array}{c}0.71 \\
(0.52,0.90)\end{array}$ & $\begin{array}{c}0.70 \\
(0.52,0.88)\end{array}$ & $\begin{array}{c}0.70 \\
(0.51,0.88)\end{array}$ \\
\hline \multicolumn{7}{|c|}{$\begin{array}{l}\text { Values in parentheses are } 95 \text { per cent confidence intervals. Residual tumour (tumour regression grade } 2-5 \text { in resected } \\
\text { primary tumour) was considered the positive outcome. } \\
\text { T2W, T2-weighted; DW, diffusion-weighted; PPV, positive predictive value; NPV, negative predictive value; AUC, area } \\
\text { under the receiver operating characteristic curve. } \\
\text { *Comparison of T2W-MRI versus T2W+DW-MRI: } P=0.441, P=0.611 \text { and } P=0.828 \text { for readers } 1,2 \text { and } 3 \\
\text { respectively (DeLong test }{ }^{33} \text { ). }\end{array}$} \\
\hline
\end{tabular}


Equivocal (confidence level 3) scores

Readers 1, 2 and 3 assigned 16, 30 and 15 equivocal scores (CLS3) respectively on T2W-MRI, which decreased to 9,11 and 9 equivocal scores after the addition of DW-MRI. Fig. 5 shows an example of a tumour for which all readers assigned an equivocal score on T2W-MRI, whereas a correct diagnosis of residual tumour (CLS4 for all 3 readers) was made after addition of DW-MRI.

Interobserver agreement

Interobserver agreement was fair to moderate on T2WMRI alone (quadratic weighted $\kappa=0.24$, 0.55 and 0.41 ), and increased to moderate to good on T2W+DW-MRI (quadratic weighted $\kappa$ $=0.55,0.71$ and 0.61 ).

Table 3. Number of test-positive and test-negative patients according to tumour regression grade for response assessment on T2-weighted combined with diffusion-weighted MRI

\begin{tabular}{|c|c|c|c|c|c|c|}
\hline \multirow{3}{*}{$\begin{array}{l}\text { Mandard } \\
\text { grade }\end{array}$} & \multicolumn{6}{|c|}{ Number of patients } \\
\hline & \multicolumn{2}{|c|}{ Reader 1} & \multicolumn{2}{|c|}{ Reader 2} & \multicolumn{2}{|c|}{ Reader 3} \\
\hline & MRI-positive & MRI-negative & MRI-positive & MRI-negative & MRI-positive & MRI-negative \\
\hline TRG 1 & 6 & 6 & 7 & 5 & 7 & 5 \\
\hline TRG 2 & 16 & 1 & 16 & 1 & 15 & 2 \\
\hline TRG 3 & 15 & 1 & 16 & 0 & 14 & 2 \\
\hline TRG 4 & 4 & 0 & 4 & 0 & 4 & 0 \\
\hline TRG 5 & 2 & 0 & 2 & 0 & 2 & 0 \\
\hline
\end{tabular}

TRG, tumour regression grade according to Mandard and colleagues;

MRI-positive, clinical residual tumour; MRI-negative, clinical complete response. 


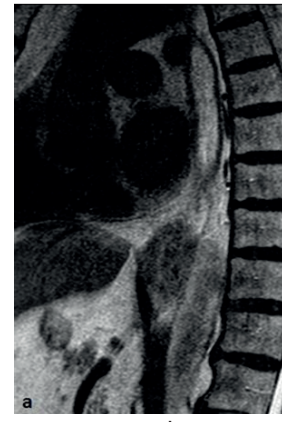

a. T2W sagittal image before $\mathrm{nCRT}$

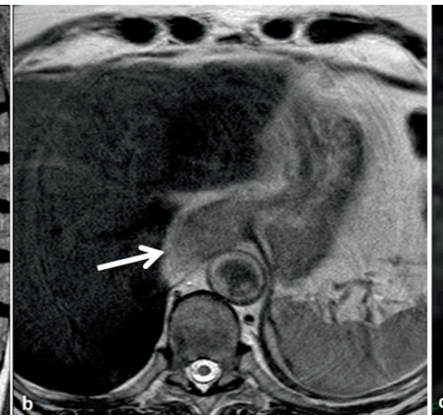

b. T2W transverse image before $\mathrm{nCRT}$

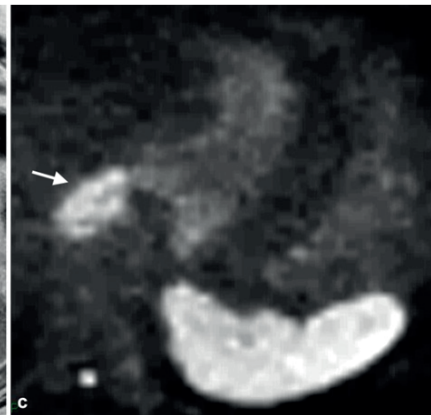

c. DW image before $\mathrm{nCRT}$

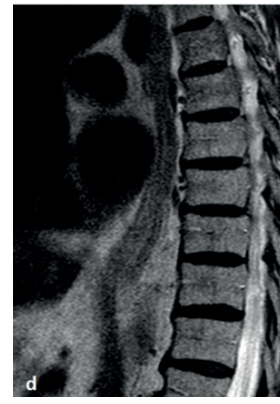

d. T2W sagittal image after nCRT

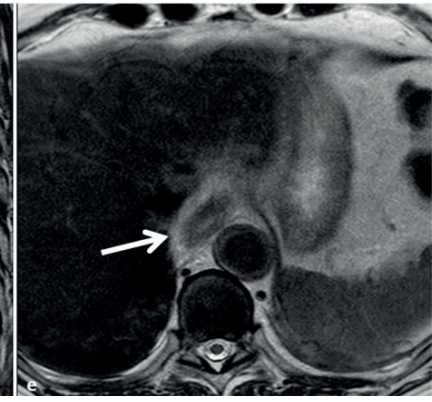

e. T2W transverse image after nCRT

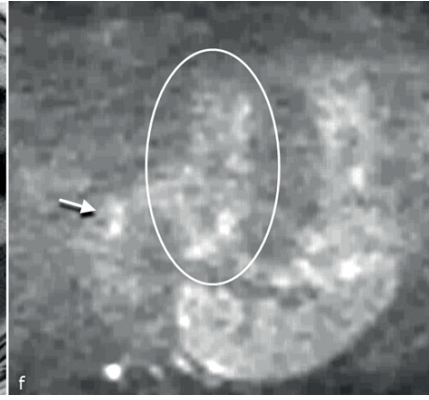

f. DW image after nCRT

Figure 5. MRI of a patient with locally advanced oesophageal cancer located at the gastrooesophageal junction Images from an 80-year-old man with a cT3 NO squamous cell carcinoma located at the gastrooesophageal junction. Histopathology after oesophagectomy showed residual tumour (tumour regression grade 2, ypTla N0). a-c T2-weighted (T2W) sagittal (a) and transverse (b) images before neoadjuvant chemoradiotherapy (nCRT) show a thick hyperintense wall, accompanied by a hyperintense signal on diffusion-weighted (DW) imaging (c). $d$-f After nCRT, the T2W images (d,e) show shrinkage of the wall with a mixed hyperintense and hypointense signal, which was assigned a confidence level score of 3 by all readers. The DW image ( $f$ ) shows spots of hyperintense signal in the primary tumour area (arrow), which is suspicious for residual tumour and was therefore assigned a confidence level score of 4 by all readers. The area within the circle indicates normal stomach wall, which also shows small hyperintense areas on DWimaging. Arrows indicate tumour location. 


\section{DISCUSSION}

This study has shown that preoperative response assessment after nCRT for oesophageal cancer performed visually on (DW-)MRI has promising overall diagnostic performance, with AUCs in the range $0.65-0.71$. The sensitivity of DW-MRI for detection of residual tumour was high lover 90 per cent), indicating that the chance of missing residual tumour was small. Addition of images obtained by functional DW-MRI to the anatomical T2W-MRI protocol did not influence the overall diagnostic performance to a great extent, but had a positive impact on the specificity and NPV for most readers. Moreover, addition of DW sequences led to improved interobserver agreement and a reduction in the number of equivocal scores, indicating increased confidence of the readers. MRI showed promising visualization of the primary oesophageal tumour bed after $\mathrm{nCRT}$ in oesophageal cancer and could thereby improve current response assessment strategies. The main drawback was the poor specificity of MRI in this unimodal approach, which in clinical practice would result in overstaging of complete responders as having residual tumour and, consequently, overtreatment. Therefore, exploration of response assessment including MRI, but also other diagnostic modalities, after nCRT for locally advanced oesophageal cancer is warranted.

The present study evaluated oesophageal MRI for the assessment of complete response of the primary tumour by visual interpretation of morphology on T2W-MRI and restrictive signals on DW-MRI. Previous studies $26,27,29$ on this subject did not perform visual assessment, but focused on quantitative DW-MRI. These studies found that an increase in the apparent diffusion coefficient (ADC, a quantitative measure of the magnitude of diffusion) during $\mathrm{nCRT}$, compared with before $\mathrm{nCRT}$, is a predictor of response. Furthermore, an increase in ADC after nCRT compared with the baseline value seemed to be predictive of gross tumour response, defined as TRG 1-228,29 or TRG $1-3^{25}$. However, the results reported for the preoperative selection of complete response (TRG 1 only) using ADC values were poor 26,29 . In contrast, in the present study, preoperative visual response assessment on DW-MRI after $\mathrm{nCRT}$ had high sensitivity for the detection of residual tumour. Moreover, the cut-off was predefined and can therefore be used prospectively, whereas in the aforementioned studies exploring ADC values, the optimal cut-offs were defined retrospectively which limits their use.

A recent study" of clinical assessment with endoscopy/EUS, in which bite-on-bite biopsies and fine-needle aspirates were obtained after $\mathrm{nCRT}$, yielded a specificity of 72 per cent for detection of residual tumour, which is higher than the specificity in the present study. However, the reported sensitivity of 77 per cent was lower than values of over 90 per cent in the present study. Combined with clinical examination and endoscopy, (DW-)MRI has led to the safe selection of patients with rectal tumours for a watch-and-wait policy after $\mathrm{nCRT}^{35}$. Combining (DW-)MRI and endoscopy/ EUS will potentially result in accurate assessment of $\mathrm{pCR}$ after $\mathrm{nCRT}$ for oesophageal cancer without missing residual disease.

The specificity for detection of residual tumour improved from 8-25 to $42-50$ per cent after adding 
DW-MRI in the present study. This specificity is, however, still low. One potential explanation for the overstaging of a $\mathrm{pCR}$ as residual tumour is the occurrence of small punctate foci of hyperintensity at the former tumour bed on images obtained with $b=800$ DW-MRI (implying restricted diffusion, which raises the suspicion of tumour). These false-positive foci were observed in patients with a tumour of the gastro-oesophageal junction and may be explained by the fact that the normal stomach wall also shows small hyperintensities on DW-MRI, or by the presence of radiationinduced inflammation. Prolonging the interval between the end of radiotherapy and MRI may result in resolution of inflammation. Furthermore, prolonging the interval between radiation and surgery may lead to an increase in pCR rates. A recent analysis in oesophageal cancer ${ }^{36}$ showed that a longer interval between $n C R T$ and surgery increased $\mathrm{PCR}$ rates, without increasing the frequency of postoperative complications.

This study had a relatively large sample size compared with previous response studies in oesophageal cancer; however, validation in a larger cohort is required. This will also allow subgroup analyses of squamous cell carcinoma and adenocarcinoma. Another potential limitation is that ADC maps were not included in the response evaluation, although the readers could always refer to the T2W images to rule out, for example, shine-through effects caused by fluid in the oesophageal lumen. Furthermore, lymph node response was not assessed for two reasons. The differentiation between benign and malignant lymph nodes on oesophageal T2W-MRI remains challenging as non-enlarged nodes may harbour malignant cells, whereas reactive (benign) nodes may be enlarged ${ }^{37,38}$. DW-MRI can detect lymph nodes, but all lymph nodes have a high signal on DW images. In patients with TON1 rectal cancer, MRI showed poor performance for detection of lymphadenopathy ${ }^{39}$. Second, the field of view (FOV) of MRI in this study focused on the primary tumour and did not comprise the complete craniocaudal perioesophageal area owing to imaging time restrictions. Hence, lymph nodes outside this FOV could not be assessed. Therefore, this study focused on tumour detection at the primary tumour bed only (TRG 1, ypTO). Other response assessment tools are needed for the detection of lymph node metastases after nCRT, such as EUS. To overcome the limitations of the present study and further increase specificity without decreasing sensitivity for the preoperative detection of residual tumour in oesophageal cancer, larger studies are needed. The multicentre observational PRIDE (Preoperative Image-guided Identification of Response to Neoadjuvant Chemoradiotherapy in Esophageal Cancer) study ${ }^{40}$ was initiated to explore the combination of multiple diagnostic modalities in assessing the response of the primary tumour and lymph nodes to chemoradiotherapy. This study aims to develop an optimal multimodal response prediction model focusing on clinical (endoscopy and EUS) and radiological (MRI and FDG-PET/CT) assessment combined with patient-specific parameters (such as circulating tumour DNA) for oesophageal cancer. 


\section{REFERENCES}

1. Shapiro J, van Lanschot JJB, Hulshof $M$, et al. Neoadjuvant chemoradiotherapy plus surgery versus surgery alone for oesophageal or junctional cancer (CROSS): long-term results of a randomised controlled trial. Lancet Oncol 2015;16:1090-8.

2. van Hagen $P$, Hulshof MC, van Lanschot JJ, et al. Preoperative chemoradiotherapy for esophageal or junctional cancer. N Engl J Med 2012;366:2074-84.

3. Mandard AM, Dalibard F, Mandard JC, et al. Pathologic assessment of tumor regression after preoperative chemoradiotherapy of esophageal carcinoma. Clinicopathologic correlations. Cancer 1994;73:2680-6.

4. Berger AC, Farma J, Scott WJ, et al. Complete response to neoadjuvant chemoradiotherapy in esophageal carcinoma is associated with significantly improved survival. J Clin Oncol 2005;23:4330-7.

5. Donahue JM, Nichols FC, Li Z, et al. Complete pathologic response after neoadjuvant chemoradiotherapy for esophageal cancer is associated with enhanced survival. Ann Thorac Surg 2009;87:392-8.

6. van der Wilk BJ, Eyck BM, Spaander MCW, et al. Towards an Organ-Sparing Approach for Locally Advanced Esophageal Cancer. Dig Surg 2018:1-8.

7. Konieczny A, Meyer P, Schnider A, et al. Accuracy of multidetector-row $C T$ for restaging after neoadjuvant treatment in patients with oesophageal cancer. Eur Radiol 2013;23:2492502.

8. Kwee RM. Prediction of tumor response to neoadjuvant therapy in patients with esophageal cancer with use of $18 \mathrm{~F}$ FDG PET: a systematic review. Radiology 2010;254:707-17.

9. van Rossum PS, Fried DV, Zhang $L$, et al. The Incremental Value of Subjective and Quantitative Assessment of 18F-FDG PET for the Prediction of Pathologic Complete Response to Preoperative Chemoradiotherapy in Esophageal Cancer. J Nucl Med 2016;57:691-700.

10. Yip C, Cook GJ, Landau DB, Davies A, Goh V. Performance of different imaging modalities in assessment of response to neoadjuvant therapy in primary esophageal cancer. Dis Esophagus 2016;29:116-30.

11. Noordman BJ, Spaander MCW, Valkema R, et al. Detection of residual disease after neoadjuvant chemoradiotherapy for oesophageal cancer (preSANO): a prospective multicentre, diagnostic cohort study. Lancet Oncol 2018;19:965-74.

12. Heneghan HM, Donohoe C, Elliot J, et al. Can CT-PET and Endoscopic Assessment PostNeoadjuvant Chemoradiotherapy Predict Residual Disease in Esophageal Cancer? Ann Surg 2016;264:831-8.

13. van Heiil M, Omloo JM, van Berge Henegouwen $\mathrm{Ml}$, et al. Fluorodeoxyglucose positron emission tomography for evaluating early response during neoadjuvant chemoradiotherapy in patients with potentially curable esophageal cancer. Ann Surg $2011 ; 253: 56-63$.

14. Griffin JM, Reed CE, Denlinger CE. Utility of restaging endoscopic ultrasound after neoadjuvant therapy for esophageal cancer. Ann Thorac Surg 2012;93:1855-9.

15. Jost C, Binek J, Schuller JC, etal. Endosonographic radial tumor thickness after neoadjuvant chemoradiation therapy to predict response and survival in patients with locally advanced esophageal cancer: a prospective multicenter phase II study by the Swiss Group for Clinical Cancer Research (SAKK 75/02). Gastrointest Endosc 2010;71:1114-21.

16. van Rossum PS, Goense L, Meziani J, et al. Endoscopic biopsy and EUS for the detection of pathologic complete response after neoadjuvant chemoradiotherapy in esophageal cancer: a systematic review and meta-analysis. Gastrointest Endosc 2016;83:866-79.

17. Chao YK, Chang Y, Yeh CJ, Chang HK, Tseng CK, Chuang WY. Characterization of residual tumours at the primary site in patients with a near pathological complete response after neoadjuvant chemoradiotherapy for oesophageal cancer. $\mathrm{Br} \mathrm{J}$ Surg 2016;103:1874-9.

18. Shapiro J, ten Kate FJ, van Hagen P, Biermann $\mathrm{K}$, Wijnhoven $\mathrm{BP}$, van Lanschot JJ. Residual esophageal cancer after neoadjuvant chemoradiotherapy frequently involves the mucosa and submucosa. Ann Surg 2013;258:678-88. 
19. Castoro C, Scarpa M, Cagol $M$, et al. Complete clinical response after neoadjuvant chemoradiotherapy for squamous cell cancer of the thoracic oesophagus: is surgery always necessary? J Gastrointest Surg 2013;17:137581 .

20. Furlong $\mathrm{H}$, Bass $\mathrm{G}$, Breathnach $O, \mathrm{O}^{\prime}$ Neill $\mathrm{B}$, Leen E, Walsh TN. Targeting therapy for esophageal cancer in patients aged 70 and over. J Geriatr Oncol 2013;4:107-13.

21. Ohkura Y, Shindoh J, Ueno M, lizuka T, Udagawa $\mathrm{H}$. Comparison of Outcome of Esophagectomy Versus Nonsurgical Treatment for Resectable Esophageal Cancer with Clinical Complete Response to Neoadjuvant Therapy. Ann Surg Oncol 2018;25:2428-33.

22. Taketa T, Xiao L, Sudo K, et al. Propensity-based matching between esophagogastric cancer patients who had surgery and who declined surgery after preoperative chemoradiation. Oncology 2013;85:95-9.

23. Lambregts DM, Vandecaveye $V$, Barbaro $B$, et al. Diffusion-weighted MRI for selection of complete responders after chemoradiation for locally advanced rectal cancer: a multicenter study. Ann Surg Oncol $2011 ; 18: 2224-31$.

24. Maas M, Lambregts DM, Nelemans PJ, et al. Assessment of Clinical Complete Response After Chemoradiation for Rectal Cancer with Digital Rectal Examination, Endoscopy, and MRI: Selection for Organ-Saving Treatment. Ann Surg Oncol 2015;22:3873-80.

25. De Cobelli F, Giganti F, Orsenigo E, et al. Apparent diffusion coefficient modifications in assessing gastro-oesophageal cancer response to neoadjuvant treatment: comparison with tumour regression grade at histology. Eur Radiol 2013;23:2165-74.

26. Fang $P$, Musall BC, Son JB et al. Multimodal Imaging of Pathologic Response to Chemoradiation in Esophageal Cancer. Int J Radiat Oncol Biol Phys 2018;102:996-1001.

27. Imanishi S, Shuto K, Aoyagi T, Kono T, Saito $H$, Matsubara $H$. Diffusion-weighted magnetic resonance imaging for predicting and detecting the early response to chemoradiotherapy of advanced esophageal squamous cell carcinoma. Dig Surg 2013;30:240-8.

28. Li QW, Qiu B, Wang B, et al. Prediction of pathologic responders to neoadjuvant chemoradiotherapy by diffusion-weighted magnetic resonance imaging in locally advanced esophageal squamous cell carcinoma: a prospective study. Dis Esophagus 2018;31.

29. van Rossum $P S$, van Lier $A L$, van Vulpen $M$, et al. Diffusion-weighted magnetic resonance imaging for the prediction of pathologic response to neoadjuvant chemoradiotherapy in esophageal cancer. Radiother Oncol 2015;115:163-70.

30. Foti PV, Privitera G, Piana S, et al. Locally advanced rectal cancer: Qualitative and quantitative evaluation of diffusion-weighted $M R$ imaging in the response assessment after neoadjuvant chemo-radiotherapy. Eur J Radiol Open 2016;3:145-52.

31. Lever FM, Lips IM, Crijns SP, et al. Quantification of esophageal tumor motion on cine-magnetic resonance imaging. Int J Radiat Oncol Biol Phys 2014;88:419-24.

32. Rice TW, Blackstone EH, Rusch WW. 7th edition of the AJCC Cancer Staging Manual: esophagus and esophagogastric junction. Ann Surg Oncol 2010;17:1721-4.

33. Delong DM, Delong ER, Clarke-Pearson DL. Comparing the Areas under Two or More Correlated Receiver Operating Characteristic Curves: A Nonparametric Approach. Biometrics $1988 ; 44: 837$

34. Cohen's unweighted kappa, kappa with linear weighting, kappa with quadratic weighting, frequencies and proportions of agreement. Published 2001, Updated 2018. Accessed July 3, 2018.

35. van der Valk MJM, Hilling DE, Bastiaannet E, et al. Long-term outcomes of clinical complete responders after neoadjuvant treatment for rectal cancer in the International Watch \& Wait Database (IWWD): an international multicentre registry study. Lancet 2018;391:2537-45.

36. van der Werf LR, Dikken JL, van der Willik $E M$, et al. Time interval between neoadjuvant chemoradiotherapy and surgery for oesophageal or junctional cancer: A nationwide study. Eur J Cancer 2018;91:76-85.

37. Alper F, Turkyilmaz A, Kurtcan S, et al. Effectiveness of the STIR turbo spin-echo sequence MR imaging in evaluation of lymphadenopathy in esophageal cancer. Eur J Radiol 2011 ;80:625-8.

38. Mizowaki T, Nishimura Y, Shimada $Y$, et al. 
Optimal size criteria of malignant lymph nodes in the treatment planning of radiotherapy for esophageal cancer: evaluation by computed tomography and magnetic resonance imaging. Int J Radiat Oncol Biol Phys 1996;36:1091-8.

39. Loftas $P$, Sturludottir $M$, Hallbook $O$, Almlov $\mathrm{K}$, Arbman G, Blomqvist L. Assessment of remaining tumour involved lymph nodes with MRI in patients with complete luminal response after neoadjuvant treatment of rectal cancer. $\mathrm{Br} J$ Radiol 2018;91:20170938.

40. Borggreve AS, Mook $S$, Verheii $M$, et al. Preoperative image-guided identification of response to neoadjuvant chemoradiotherapy in esophageal cancer (PRIDE): a multicenter observational study. BMC cancer 2018;18:1006. 
DIAGNOSTIC PERFORMANCE OF MRI FOR ASSESSMENT OF RESPONSE TO NEOADJUVANT CHEMORADIOTHERAPY 


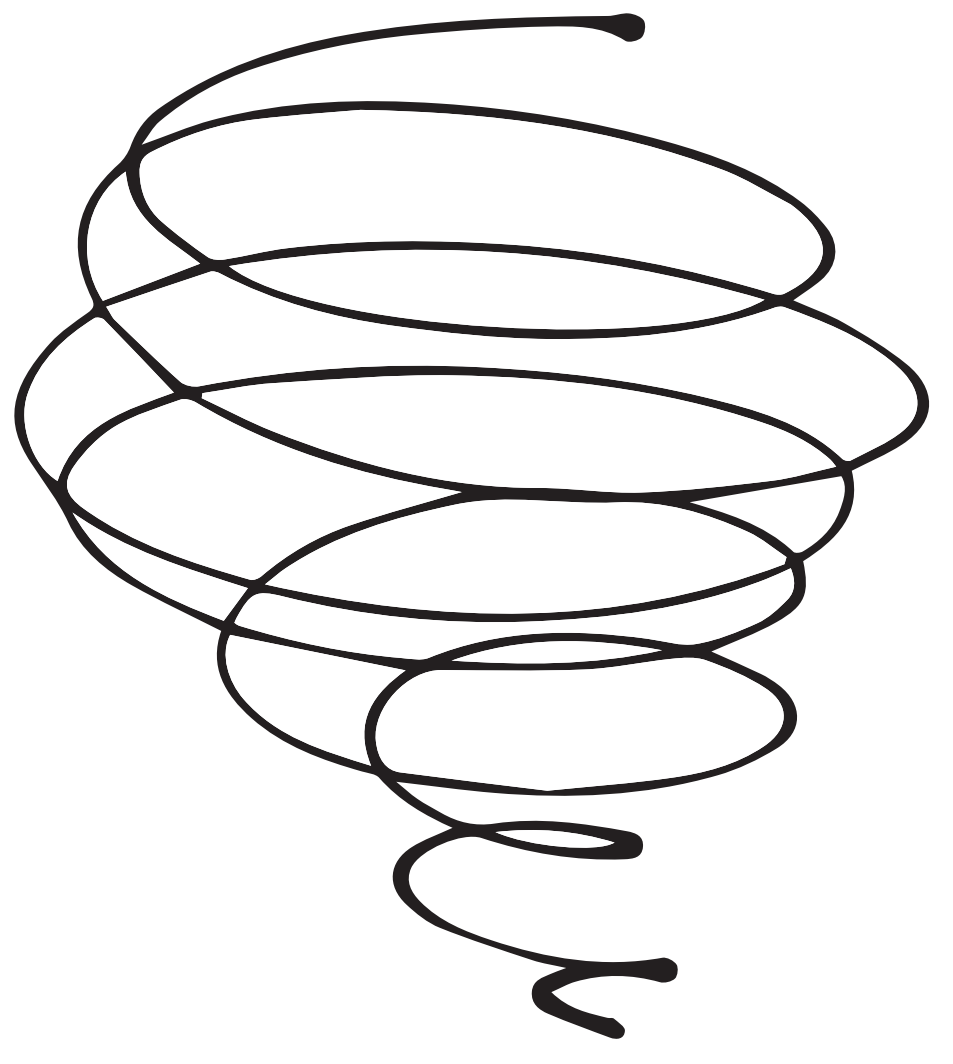




\section{CHAPTER 3}

\section{Diffusion-weighted MRI with ADC mapping for response prediction and assessment of oesophageal cancer: A systematic review}

Radiotherapy and Oncology; Jan 2020 (Volume 142, p17-26)

Sophie E. Vollenbrock

Francine E.M. Voncken Lambertus W. Bartels

Regina G.H. Beets-Tan Annemarieke Bartels-Rutten 


\begin{abstract}
Purpose

The aim was to perform a systematic review on the value of diffusion-weighted MRI (DW-MRI) with apparent diffusion coefficient (ADC) mapping in the prediction and assessment of response to chemo- and/or radiotherapy in oesophageal cancer.
\end{abstract}

Materials and methods

A systematic search was performed on Pubmed, Embase, Medline and Cochrane databases. Studies that evaluated the ADC for response evaluation before, during or after chemo- and/or radiotherapy were included. The Quality Assessment of Diagnostic Accuracy Studies (QUADAS-2) was used to assess the quality of the included studies.

Results

Fourteen studies, comprising 516 patients, in which the response to treatment in oesophageal cancer was evaluated on ADC maps were included. Acquisition parameter settings for DWMRI and ROI placement varied substantially. The reference standard was RECIST or endoscopic assessment in eight non-surgery studies and histopathology after surgery in six studies. A high pretreatment $A D C$ significantly correlated with good response in three out of 12 studies; conversely, one study reported a significantly higher pre-treatment $A D C$ in poor responders. In five out of eight studies good responders showed a significantly larger relative increase in ADC two weeks after the onset of treatment (range 23-59\%) than poor responders (range 1.5-17\%). After chemo- and/or radiotherapy $A D C$ results varied considerably, amongst others due to large variation in the interval between completion of therapy and DW-MRI.

Conclusion

DW-MRI for response evaluation to chemo- and/or radiotherapy in oesophageal cancer shows variable methods and results. A large relative ADC increase after two weeks of treatment seems most predictive for good response. 


\section{INTRODUCTION}

Oesophageal cancer is a malignancy with a poor prognosis, as reflected by an average overall 5 -year survival of only 18\%'. Locally advanced oesophageal cancer patients are generally treated by chemo- and/or radiotherapy, either as definite treatment or as neoadjuvant treatment prior to surgery ${ }^{2-4}$. However, not all patients benefit equally. In $23 \%$ of adenocarcinomas (AC) and $49 \%$ of squamous cell carcinomas (SCC) a pathological complete response ( $\mathrm{PCR}$, tumour regression grade (TRG) 1) is determined on histopathology of the resection specimen after neoadjuvant chemoradiotherapy (nCRT) 2,5 . On the other hand, after nCRT and surgery $18 \%$ of patients have TRG 4 or 5 , meaning $>50 \%$ vital residual tumour cells in the primary tumour bed ${ }^{2,5}$.

Accurate early prediction of poor response to chemo- and/or radiotherapy using imaging techniques could allow for early adjustments in a patient's treatment plan. Thereby, unnecessary toxicity of the chemo- and/or radiotherapy can potentially be avoided ${ }^{6}$. Some studies on $18 \mathrm{~F}$ fluorodeoxyglucose positron emission tomography (FDG-PET/CT) have shown that tumours in non-responders demonstrate a smaller change in maximum standardized uptake value (SUVmax) compared to responders after 14 days of $\mathrm{nCRT}^{7,8}$. However, the optimal threshold SUVmax value for prediction is still uncertain and varies between the studies, which limits its clinical applicability ${ }^{9-11}$. Another study showed that the standardized uptake ratio (SUR) was associated with overall survival, whereas this parameter was not associated with locoregional tumour control ${ }^{12}$.

In the group of patients who respond well to chemo- and/or radiotherapy the complete responders may benefit from a nonsurgical approach. These patients have a significantly improved long-term survival compared to patients with residual tumour ${ }^{3,13,14}$. In order to assess a clinical complete response (CCR) after neoadjuvant treatment, endoscopy and endoscopic ultrasound (EUS) are currently being examined ${ }^{13-17}$. However, previous studies report false negative results (cCR in TRG $2-5$ tumours) in $28-4 \%^{13,15-17}$. The predictive value of FDG-PET/CT after CRT is lower compared with its value during CRT, potentially due to radiation-induced inflammation that masks the actual disappearance of tumour metabolic activity ${ }^{18,19}$. This encourages the exploration of the use of other imaging techniques.

In recent years, prediction and assessment of response using magnetic resonance imaging (MRI) and specifically diffusion-weighted MRI (DW-MRI) in oesophageal cancer has increasingly been explored. From the DW-MR images apparent diffusion coefficient (ADC) maps can be generated, representing a quantitative measure of water diffusivity in tissue ${ }^{20}$. The quantification of the ADC before, during and/or after (neoadjuvant) chemo- and/or radiotherapy potentially enables a more personalized treatment approach for locally advanced oesophageal cancer patients ${ }^{21}$.

The objective of this study was to systematically review the evidence for the use of the ADC values in locally advanced oesophageal cancer response prediction and assessment to (neoadjuvant) chemo- and/or radiotherapy. 


\section{METHODS}

\section{Literature search}

A systematic literature search was performed in the Pubmed, Embase and the Cochrane Library electronic databases from January 2000 until February 2019. The following search terms were used (including synonyms and related words): 'oesophageal cancer', 'magnetic resonance imaging', 'treatment response'. The full search strategy can be found in the electronic supplementary material (Supp. Table 1). The literature search and study selection were independently performed by two reviewers ( $S V$ and $A B$ ). Any discrepancies were resolved through a consensus discussion afterwards. Reviews and conference contributions were excluded.

\section{Inclusion and exclusion criteria}

The inclusion criteria were as follows: (1) studies including patients with histologically confirmed diagnosis of oesophageal cancer, (2) patients underwent DW-MRI, (3) ADC used for response prediction or evaluation, (4) the reference standard comprises tumour response to therapy: either assessed at histopathology after esophagectomy, or measured by the Response Evaluation Criteria in Solid Tumours (RECIST) on follow-up scanning in patients who did not undergo surgery ${ }^{22,23}$. In case of overlapping study populations, the study with the largest study population was included.

\section{Identification of eligible studies}

The literature search yielded a total of 1337 papers after removal of duplicates. Based on title 1185 articles were excluded, because they were not relevant to the study question. 119 articles were excluded after reading the abstract, because they either did not focus on oesophageal cancer and/or DW-MRI. The remaining 33 papers were studied in full text. Of these, 19 studies were excluded $^{19-44}$. The reasons for exclusion are outlined in Fig. 1. Eventually 14 studies were included. Literature selection results are displayed in accordance with the preferred reporting items for systematic reviews and meta-analyses (PRISMA) guidelines in Fig. $1^{24}$.

\section{Data extraction and quality assessment}

Fourteen relevant papers were included. Selected studies were evaluated for methodological quality using the Quality Assessment of Diagnostic Accuracy Studies-2 (QUADAS-2 criteria) ${ }^{25}$. A metaanalysis was not performed because of the heterogeneity of the studies. Information of the MRI field strength, the chosen b-values and definition of the region of interest (ROI) was extracted per study. The treatment characteristics were classified as neoadjuvant chemotherapy $(\mathrm{nCTx})$, neoadjuvant chemoradiotherapy (nCRT), definitive radiotherapy (dRT) or definitive chemoradiotherapy (dCRT). Furthermore, data on the evaluation of response before, during and after treatment including diagnostic performance and ADC values were extracted. 


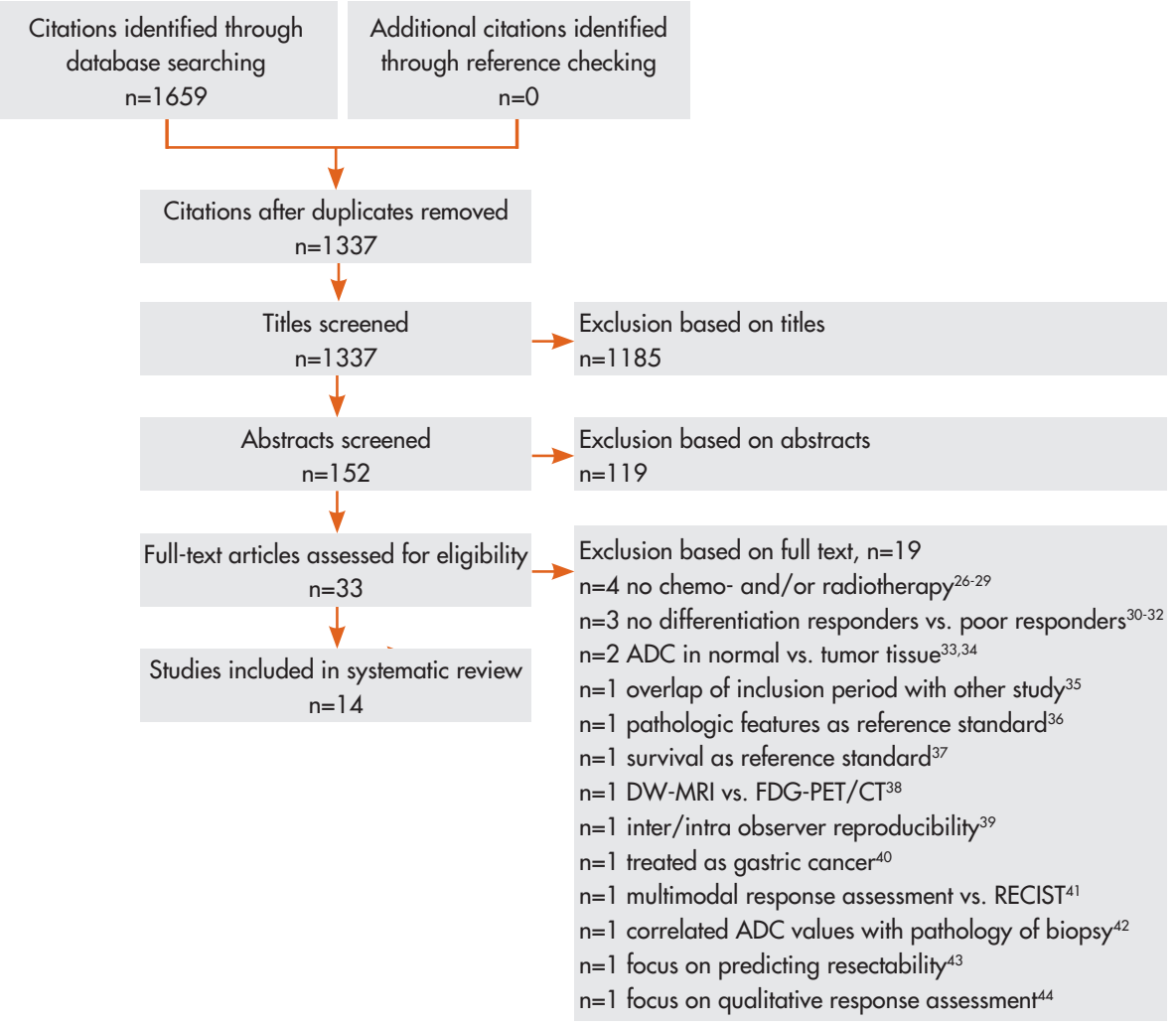

Flgure 1. PRISMA flowchart describing the identification and inclusion of the studies

\section{RESULTS}

General study characteristics

Fourteen studies, published between 2011 and 2018, included a total of 516 patients (356 SCC, 96 AC, 2 adenosquamous carcinoma, 62 unknown histology) and measured the ADC to evaluate response. The basic characteristics regarding field strength, MRI acquisition parameter settings for diffusion weighting and ROI placement are shown in Table 1. MRI scans were performed on a field strength of 1.5 Tesla in nine studies ${ }^{45-53}$. 3.0 Tesla MRI scanners were used in four studies ${ }^{54-57}$. One study did not report on the field strength used ${ }^{58}$. Furthermore, 12 out of 14 studies delineated the $\mathrm{ROI}$ on DWI and $b$-values used to calculate the ADC varied between $b=0$ and $b=1000$ (Table 1). 
Table 1. Field strength, MRI acquisition parameter settings for diffusion weighting and ROI placement

\begin{tabular}{|c|c|c|c|c|c|}
\hline & $\begin{array}{l}\mathrm{b} \text {-values }\left(\mathrm{s} / \mathrm{mm}^{2}\right) ; \\
\text { field strength } M R I\end{array}$ & $\begin{array}{l}\text { Delineation of } \\
\text { ROI on }\end{array}$ & $\begin{array}{l}\text { Excluded from } \\
\text { ROI }\end{array}$ & $\begin{array}{l}\text { Definition of } \\
\text { ROI in case } \\
\text { of complete } \\
\text { remission }\end{array}$ & $\begin{array}{l}\text { ROI adjusted } \\
\text { per time point }\end{array}$ \\
\hline \multicolumn{6}{|c|}{ Reference standard $=$ RECIST } \\
\hline $\begin{array}{l}\text { Aoyagi, } \\
2011^{45}\end{array}$ & $\begin{array}{c}\mathrm{b}=0,1000 ; \\
1.5 \mathrm{~T}\end{array}$ & DWI & NR & n.a. ${ }^{*}$ & n.a. ${ }^{*}$ \\
\hline $\begin{array}{l}\text { Imanishi, } \\
2013^{47}\end{array}$ & $\begin{array}{c}\mathrm{b}=0,1000 ; \\
1.5 \mathrm{~T}\end{array}$ & DWI & NR & NR & Yes \\
\hline Liv, $2016^{54}$ & $\begin{array}{c}\mathrm{b}=0,800 ; \\
3.0 \mathrm{~T}\end{array}$ & DWI & $\begin{array}{c}\text { Vessels and } \\
\text { necrotic areas }\end{array}$ & n.a. ${ }^{*}$ & n.a. ${ }^{*}$ \\
\hline $\begin{array}{l}\text { Wang, } \\
2016^{58}\end{array}$ & $\begin{array}{c}\mathrm{b}=0,600 ; \\
\text { TNR }\end{array}$ & DWI & NR & $\begin{array}{c}\text { DWI based } \\
\text { volume recorded } \\
\text { as } 0\end{array}$ & Yes \\
\hline $\begin{array}{l}\text { Kozumi, } \\
2018^{56}\end{array}$ & $\begin{array}{c}\mathrm{b}=50,800 ; \\
3.0 \mathrm{~T}\end{array}$ & DWI & Lumen & n.a. ${ }^{*}$ & n.a. ${ }^{*}$ \\
\hline Li, $2018^{52}$ & $\begin{array}{c}\mathrm{b}=0,10,20,30,50 \\
80,100,150,200 \\
400,600,800 ; 1.5 \mathrm{~T}\end{array}$ & DWI & $\begin{array}{l}\text { Necrosis, air and } \\
\text { vessels }\end{array}$ & $\begin{array}{l}\text { ROls at initial } \\
\text { tumour location }\end{array}$ & Yes \\
\hline $\begin{array}{l}\text { Zheng, } \\
2018^{57}\end{array}$ & $\begin{array}{c}b=0,25,50,75 \\
100,150,200,500 \\
800 ; 3.0 \mathrm{~T}\end{array}$ & DWI & $\begin{array}{l}\text { Necrosis, cystic } \\
\text { degeneration, } \\
\text { adjacent tissues }\end{array}$ & $\begin{array}{l}3 \text { identical ROIs } \\
\text { at initial tumour } \\
\text { location }\end{array}$ & Yes \\
\hline $\begin{array}{l}\text { Guo, } \\
2018^{50} \dagger\end{array}$ & $\begin{array}{c}\mathrm{B}=0,600,800, \\
1000 ; 1.5 \mathrm{~T}\end{array}$ & DWI & NR & NR & NR \\
\hline \multicolumn{6}{|c|}{ Reference standard = Histopathology } \\
\hline $\begin{array}{l}\text { De Cobelli, } \\
2013^{46}\end{array}$ & $\begin{array}{c}\mathrm{b}=0,600 ; \\
1.5 \mathrm{~T}\end{array}$ & $\begin{array}{c}\text { T2-weighted } \\
\text { MRI }\end{array}$ & Necrosis & NR & Yes \\
\hline $\begin{array}{l}\text { Weber, } \\
2013^{48}\end{array}$ & $\begin{array}{c}\mathrm{b}=50,400,800 ; \\
1.5 \mathrm{~T}\end{array}$ & MRI & Necrosis & NR & Yes \\
\hline $\begin{array}{l}\text { Kwee, } \\
2014^{49}\end{array}$ & $\begin{array}{c}\mathrm{b}=0,300,1000 ; \\
1.5 \mathrm{~T}\end{array}$ & DWI & NR & NR & Yes \\
\hline $\begin{array}{l}\text { Fang, } \\
2018^{55}\end{array}$ & $b=0,200,800 ; 3.0 T$ & DWI & $\begin{array}{l}\text { Lumen (only } \\
\text { in volume } \\
\text { contouring; not in } \\
\text { slice contouring) }\end{array}$ & NR & Yes \\
\hline $\mathrm{Li}, 2018^{53}$ & $\begin{array}{l}\mathrm{b}=0,700 ; \\
\quad 1.5 \mathrm{~T}\end{array}$ & DWI & $\begin{array}{c}\text { Necrotic, } \\
\text { cystic and } \\
\text { haemorrhagic } \\
\text { changes }\end{array}$ & $\begin{array}{l}\text { ROI at initial } \\
\text { tumour location }\end{array}$ & Yes \\
\hline $\begin{array}{l}\text { Heethuis, } \\
2018^{51}\end{array}$ & $\begin{array}{c}\mathrm{b}=0,200,800 ; \\
1.5 \mathrm{~T}\end{array}$ & DWI & NR & NR & $\begin{array}{l}\text { Yes (only } \\
\text { circumferen- } \\
\text { tial margins } \\
\text { adjusted) }\end{array}$ \\
\hline \multicolumn{6}{|c|}{$\begin{array}{l}\text { Abbreviations: } \mathrm{ROI}=\text { region of interest; CRT = chemoradiotherapy; RECIST = Response Evaluation Criteria In Solid } \\
\text { Tumours; NR = not reported; } A D C=\text { apparent diffusion coefficient. } \\
\text { * This study focused on MRI acquisition before the start of treatment only (therefore the ADC was measured on only one } \\
\text { MRI scan). } \\
\text { † This study used clinical complete response on endoscopy as the reference standard. }\end{array}$} \\
\hline
\end{tabular}


Quality of the included studies

The QUADAS-2 checklist results are shown in the electronic supplementary material (Supp. Fig. $1)^{25}$. The risk of bias regarding patient selection was high in one study, which only included cT4 tumours ${ }^{47}$. Patient selection was scored unclear if an inclusion period and inclusion number were reported without explaining potential exclusions from analyses $25,50,54-56$.

One study did not report the reference standard. Therefore, the study applicability was scored 'unclear' ${ }^{54}$. However, in this study none of the patients underwent surgery thus response was probably determined on imaging after treatment. Another study reported a clinical complete response on endoscopic assessment as the reference standard. This is not commonly used and was therefore scored as a high concern regarding applicability ${ }^{50}$.

Four studies had high risk of bias concerning flow and timing. These studies evaluated the treatment effect by RECIST on imaging immediately after completion of CRT ${ }^{47,50,52,58}$. This was considered bias because of the considerable chance of wall thickening due to radiation-induced esophagitis shortly after completion of (chemo-)radiotherapy. Another four studies were scored to have 'unclear' risk of bias concerning flow and timing. In two studies this was unclear because the interval between the end of treatment and the reference standard was not reported ${ }^{45,54}$. One study did not report on the interval between the MRI and surgery ${ }^{51}$. Lastly, in one study the treatment strategy was changed based on FDG-PET/CT scans obtained after 14 days $^{48}$.

\section{Prediction of response before treatment}

Twelve studies evaluated pre-treatment ADC for the prediction of response after treatment, shown in Table 2 and in the electronic supplementary material (Supp. Fig. 2) 45,47-50,52-58. A good response was determined in the histopathologic specimen, or using RECIST in patients who did not undergo surgery. Good response was either classified as complete response only or as complete and partial response together. The definition of a good response per study is displayed in Table 2.

Of the studies evaluating the pre-treatment $A D C$, three found a significantly higher pre-treatment mean $A D C$ in patients with a good response compared to patients with a poor response ${ }^{45,48,58}$. Conversely, one study reported a significantly lower mean $A D C$ in patients with complete and partial response $(C R+P R$, according to RECIST) versus patients with stable and progressive disease (SD + PD, according to RECIST) ${ }^{52}$. Furthermore, the use of percentiles (i.e. P25, P50, P75 and P90) of the pretreatment $A D C$ to predict treatment outcome did not result in significant outcomes 55,56 . In one study the pre-treatment $A D C$ yielded an accuracy of $80 \%$ for predicting $C R+P R$ laccording to RECIST) using a cut-off value of $1.10 \times 10^{-3} \mathrm{~mm}^{2} / \mathrm{s}^{45}$. With a cut-off value of $1.74 \times 10^{-3} \mathrm{~mm}^{2} / \mathrm{s}$ for mean ADC before treatment, the area under the curve (AUC) for predicting $C R+P R$ was 0.82 in another study ${ }^{52}$. A third study did not report a cut-off value but found that the mean $A D C$ predicted PCR (TRG1) with an AUC of $0.87^{55}$. 
Prediction of response during treatment

Response prediction during treatment was reported in nine studies, summarized in Table 3 and

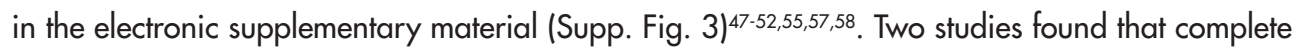
responders determined with RECIST had a significantly higher ADC during treatment as compared to patients with $\mathrm{PR}, \mathrm{SD}$ or $\mathrm{PD}^{57,58}$. Also, one study reported a significantly higher $\mathrm{ADC}$ during treatment in patients with clinical complete response on endoscopy compared to patients with residual tumour on endoscopy ${ }^{50}$. Imanishi et al. stated that the absolute ADC measured after 10 fractions (20 Gray) of radiotherapy was significantly higher in CR + PR patients than in patients with $S D+P D^{47}$. Contrarily, another study reported a significantly lower ADC during treatment for $C R+P R$ patients as compared to SD + PD patients ${ }^{52}$.

Eight out of nine studies also reported on the relative increase in $A D C$ measured during (per)

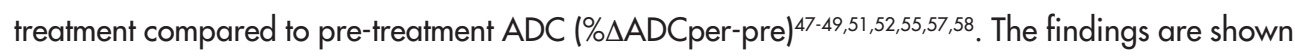
in Fig. 2. Five reported a significantly higher relative change in ADCper-pre in good versus poor responders ${ }^{47,51,52,55,57}$. In the aforementioned studies, the good responders had an ADC increase ranging from $23.5 \%$ to $58.7 \%$, whereas poor responders had an $A D C$ increase ranging from $1.5 \%$ to $16.7 \%$. Fang et al. even reported that the relative change of the mean ADC for the tumour volume predicted TRG1 with a perfect AUC of 1.0, using $28 \%$ as the cut-off ${ }^{55}$. Another study reported an AUC of 0.70 for predicting TRG1 + 2 using the 90th percentile of ADC, however, no cut-off value was reported ${ }^{51}$. Three out of eight studies did not find a difference in the ADC increase during treatment between good responders and poor responders ${ }^{48,49,58}$.

Assessment of response after treatment

The eight studies reporting on ADC values after treatment in the assessment of response to therapy are depicted in Table 4 and in the electronic supplementary material (Supp. Fig. 4) 46,47,50-53,57,58. The ADC on DW-MRI acquired directly after dCRT was significantly higher in good responders compared to poor responders in three out of five studies reporting on the ADC at this time point ${ }^{47,50,58}$. Two recent studies did not confirm these results ${ }^{52,57}$. However, one of these did show a significantly higher $A D C$ in good responders compared to poor responders one month after completion of CRT $(p=0.023)^{57}$. Seven out of eight studies reported on the relative ADC increase after (post) CRT compared to pre-treatment ADC (\% $\triangle \mathrm{ADCpost-pre)})^{46,47,51-53,57,58}$. This is summarized in Table 4 and in Supp. Fig. 5. Three studies found significant differences in the relative ADC increase after treatment compared to before treatment for variable definitions of responder groups (77\% in CR+PR versus $14.7 \%$ in PD+SD ${ }^{47} ; 171.3 \%$ in TRG $1+2+3$ versus $-11.56 \%$ in TRG4+ ${ }^{46}$; $45 \%$ in TRG1 +2 versus $23.1 \%$ in TRG3+ (90th percentile $\left.{ }^{51}\right)$ ). Furthermore, one study reported a significant difference between TRG1+2 versus TRG3+ regarding the absolute ADC increase after treatment (increase of $1.22 \times 10^{-3} \mathrm{~mm}^{2} / \mathrm{s}$ vs. $0.64 \times 10^{-3} \mathrm{~mm}^{2} / \mathrm{s}$, respectively, $p=0.007^{53}$ ). The remaining three studies did not find significant differences for the relative increase of ADC after treatment in good responders versus poor responders $52,57,58$. 


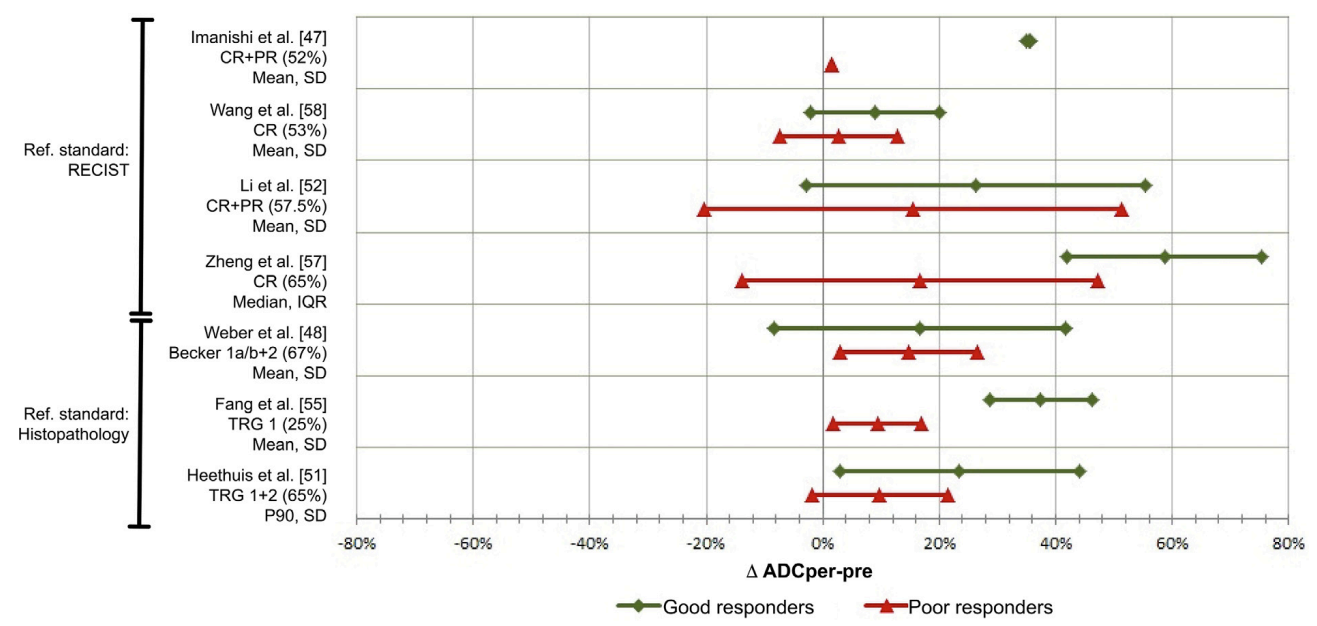

Flgure 2. Response prediction during treatment: relative difference in $A D C$ before (pre) and during (per) treatment (\% $\triangle \mathrm{ADCp}$ per-pre) for good responders versus poor responders.

Abbreviations: RECIST = Response Evaluation Criteria In Solid Tumours ${ }^{23} ; A D C=$ apparent diffusion coefficient; $C R=$ complete response according to $R E C I S T ; P R=$ partial response according to RECIST; Becker = histology according to the Becker score ${ }^{68} ;$ TRG = tumour regression grade according to Mandard 5 ; SD = standard deviation; P90 = 90th Percentile.

The names represent first authors of included studies, followed by the definition of a good responder and the proportion of good responders in the particular study. 


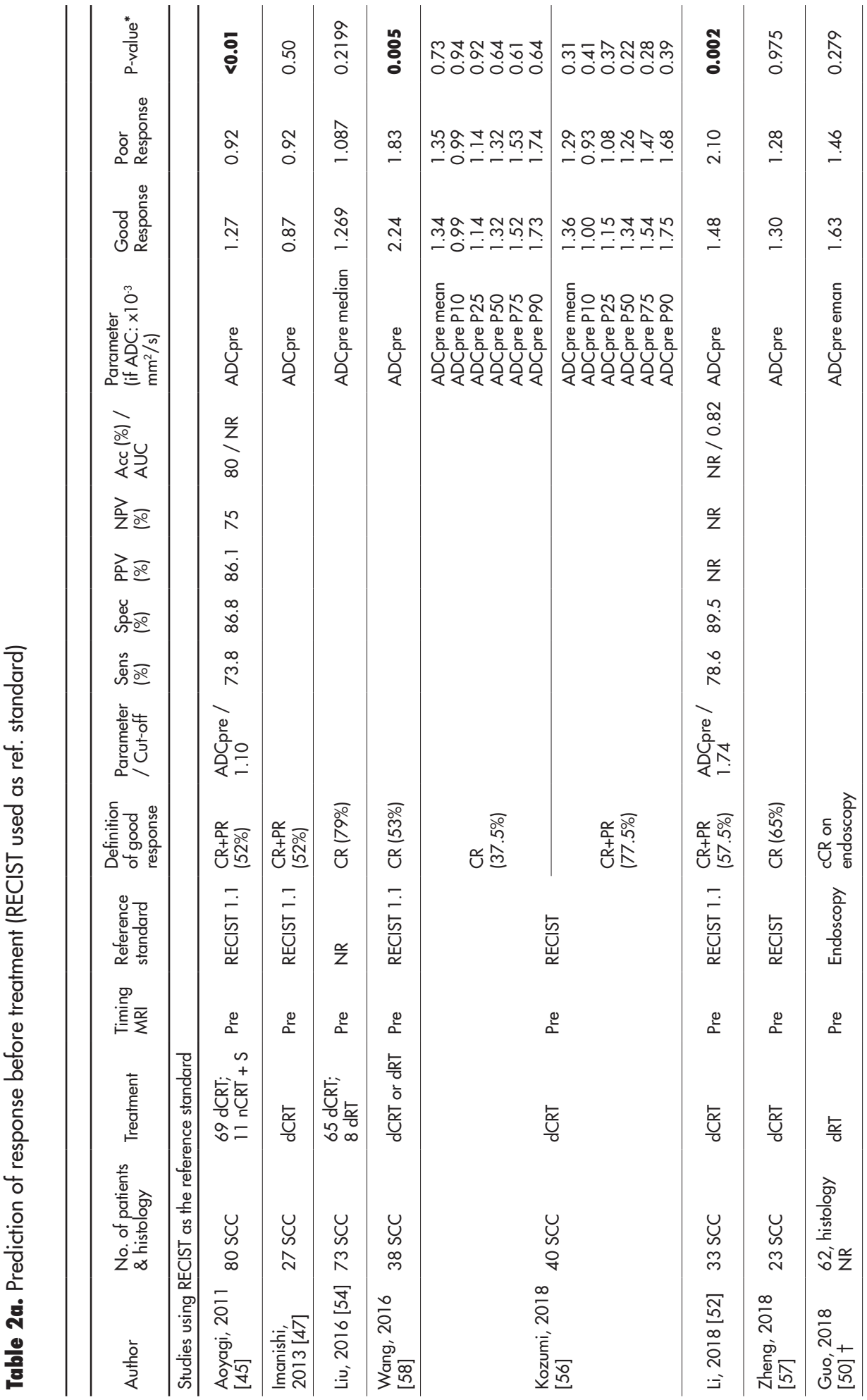


DIFFUSION-WEIGHTED MRI WITH ADC MAPPING FOR RESPONSE PREDICTION: A SYSTEMATIC REVIEW

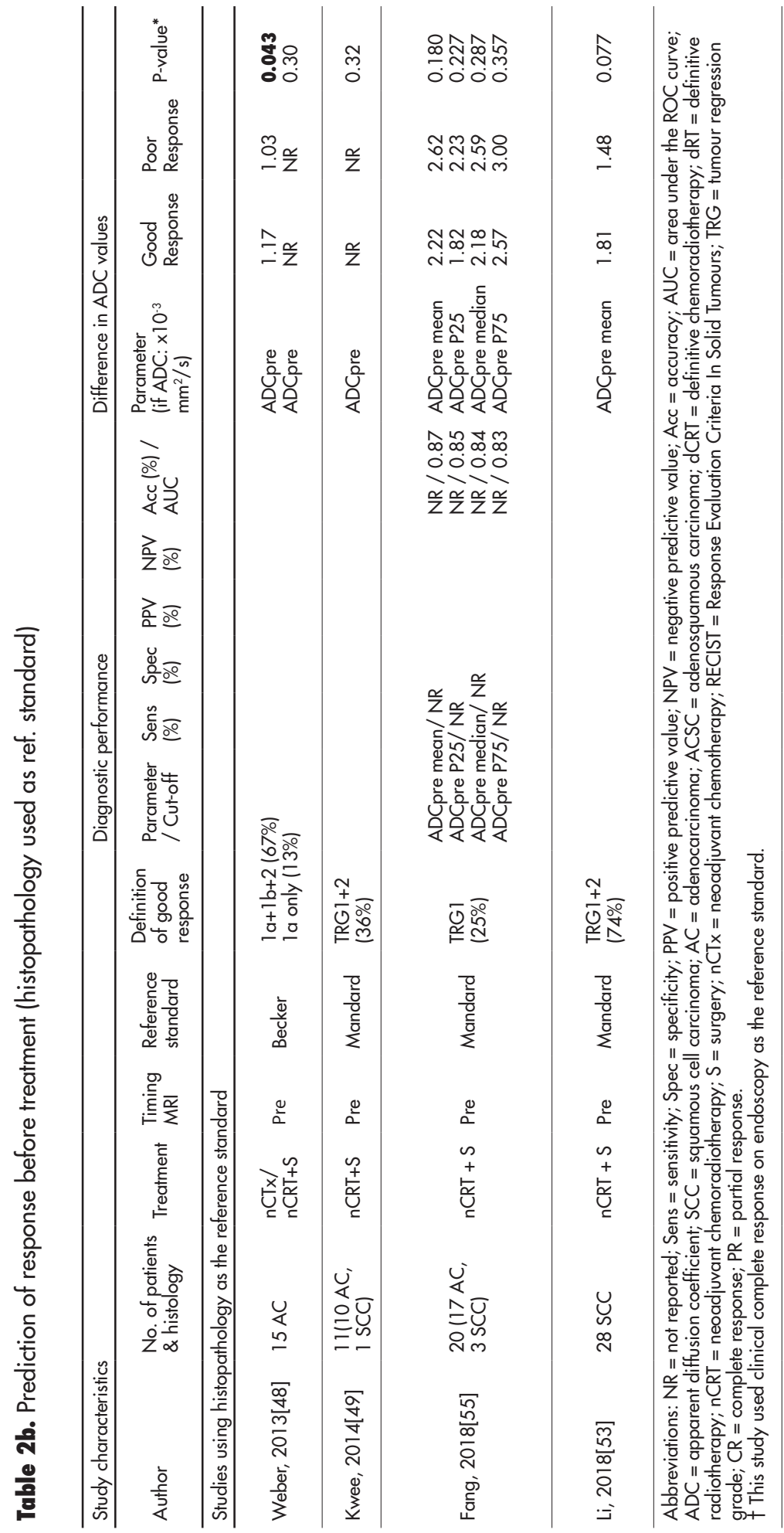




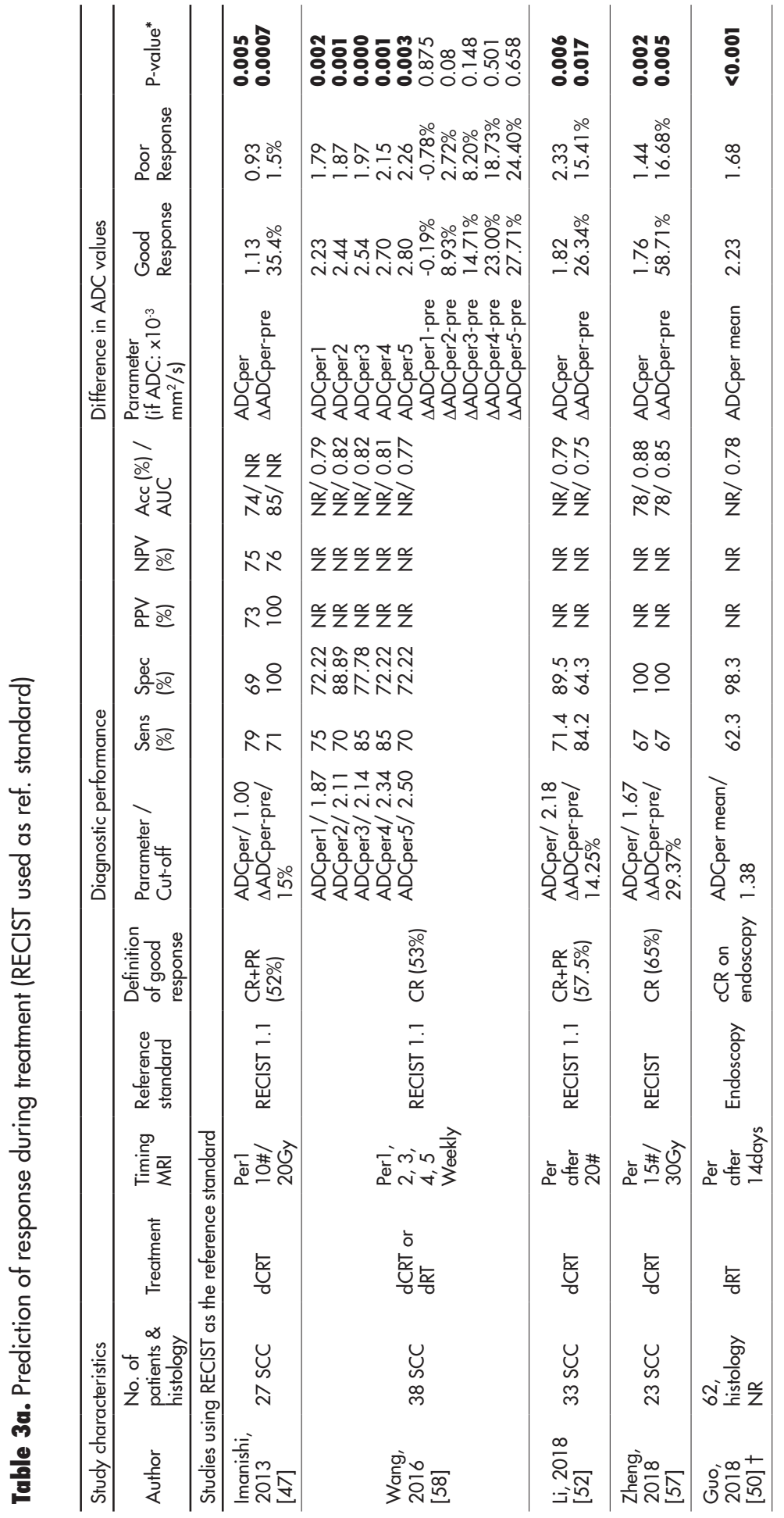


DIFFUSION-WEIGHTED MRI WITH ADC MAPPING FOR RESPONSE PREDICTION: A SYSTEMATIC REVIEW

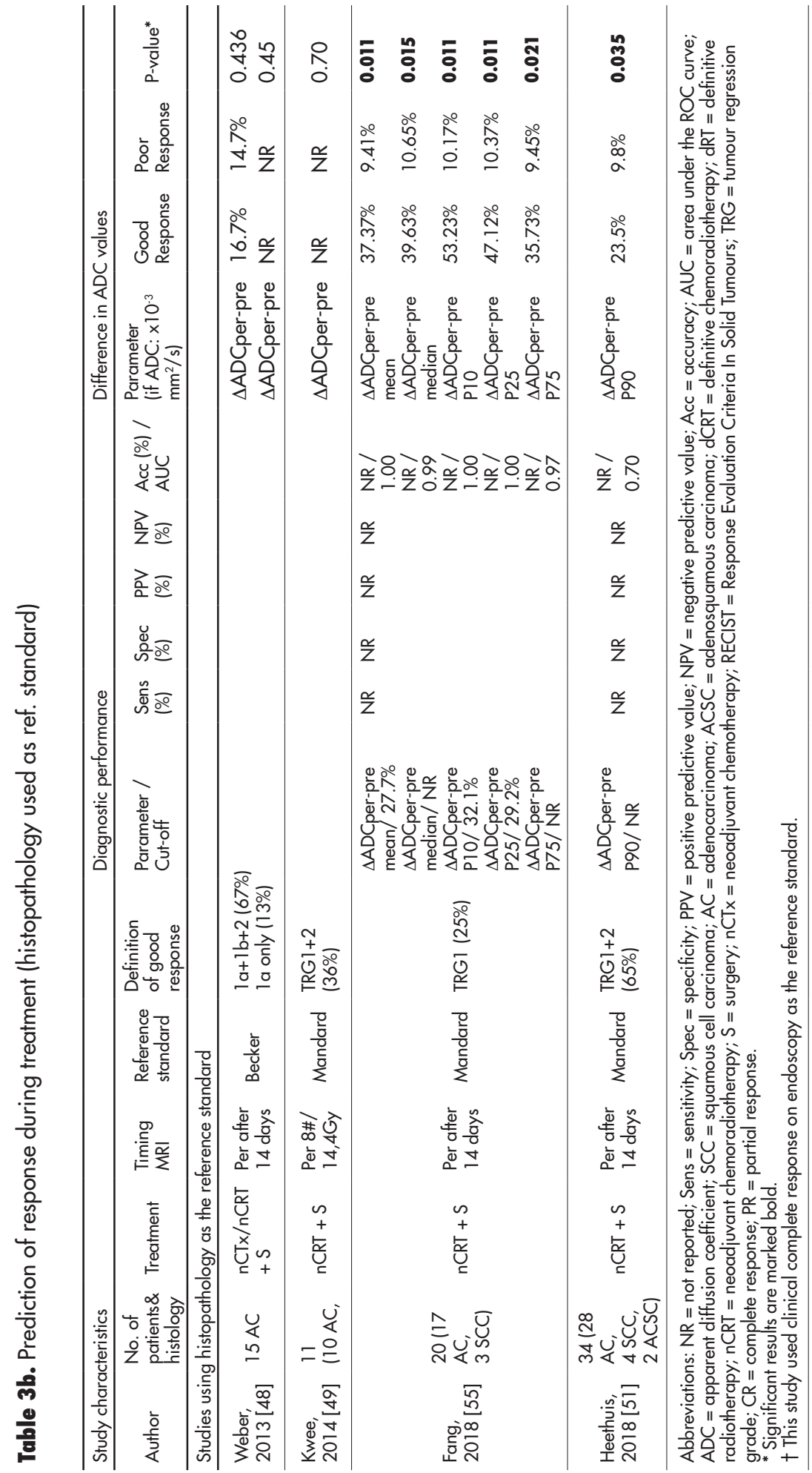




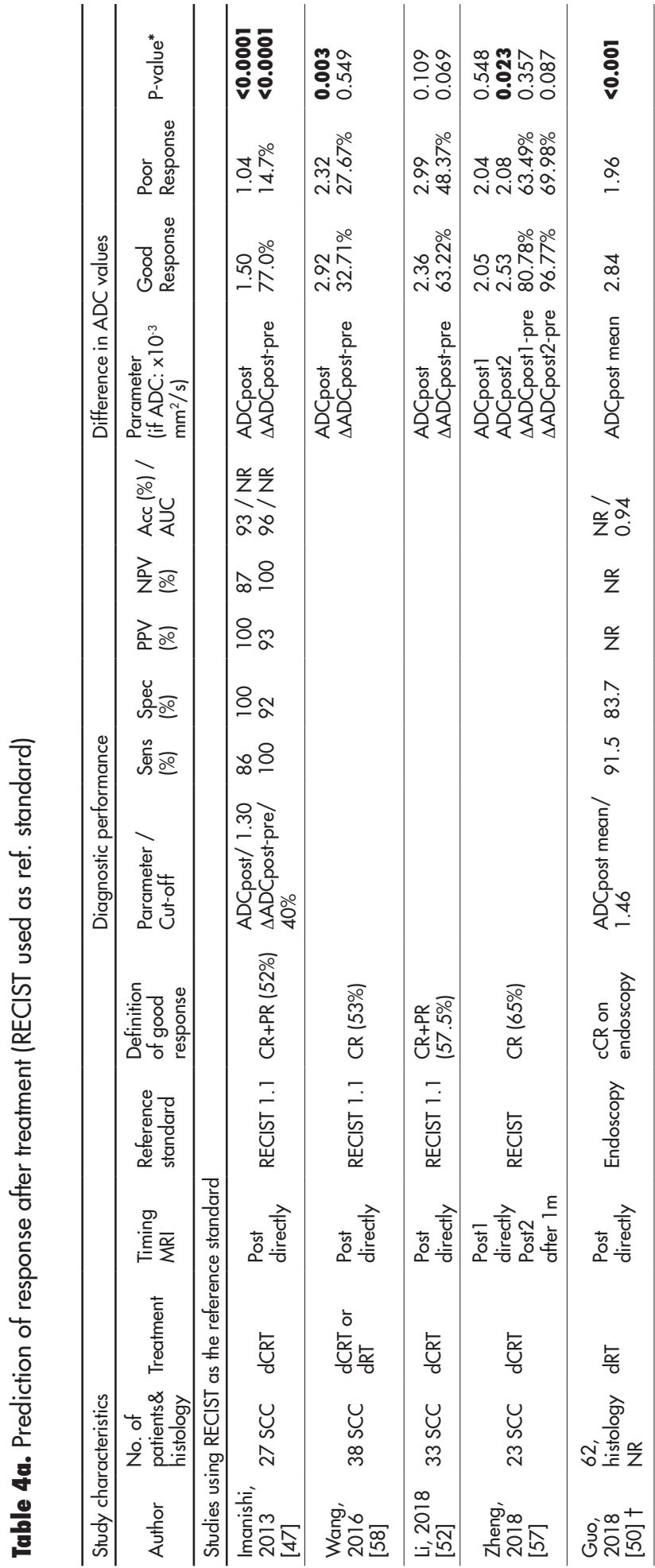


DIFFUSION-WEIGHTED MRI WITH ADC MAPPING FOR RESPONSE PREDICTION: A SYSTEMATIC REVIEW

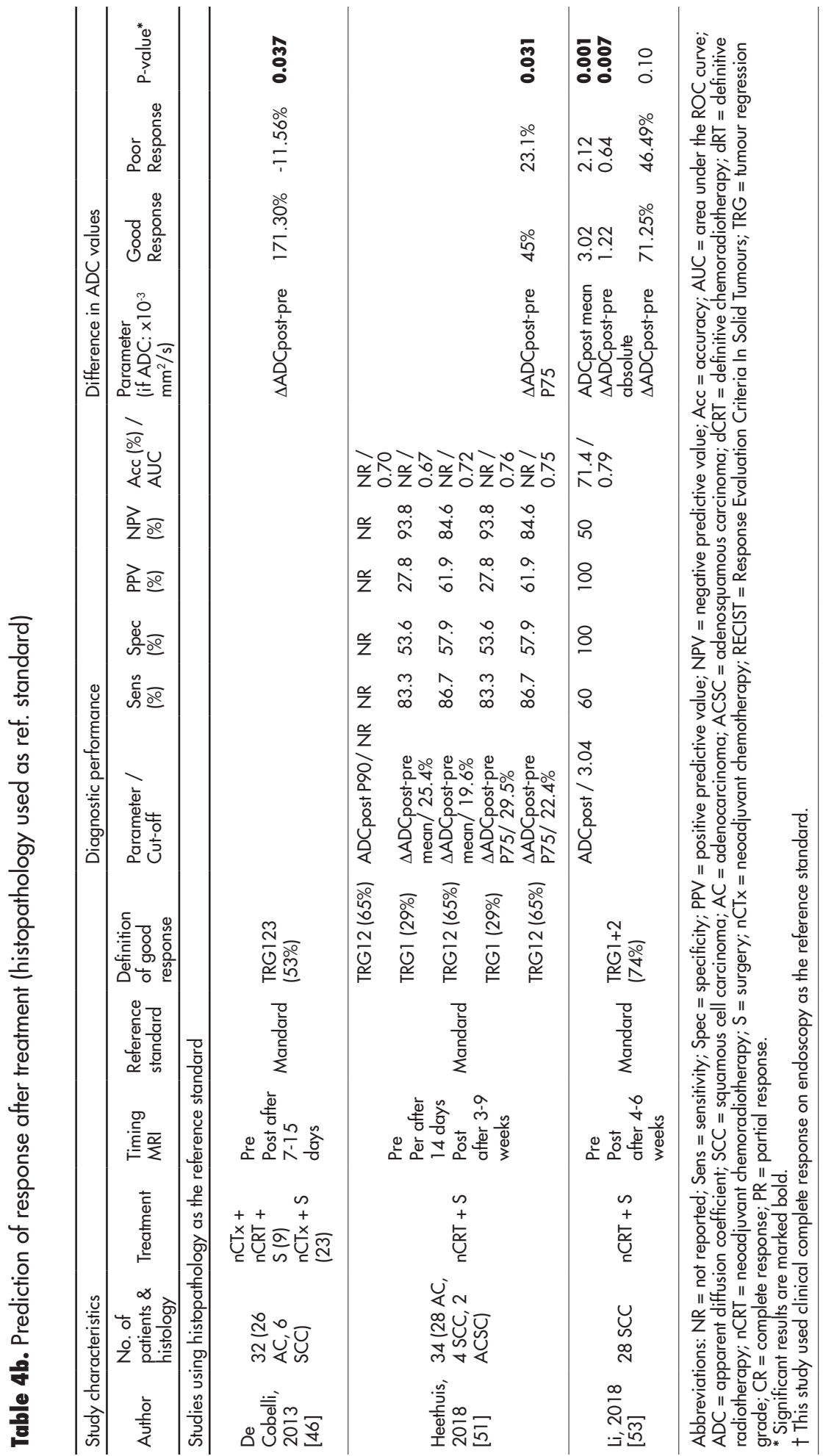




\section{DISCUSSION}

This systematic review shows that response prediction and assessment with quantification of (changes in) ADC values on DW-MRI in oesophageal cancer patients is challenging. One of the greatest challenges in interpreting quantitative measurements is dealing with the practice variations regarding the image acquisition, e.g. the b-values applied to calculate the $A D C$, and the measurement technique, e.g. the ROI placement. The relative $A D C$ change (\% $\triangle A D C)$ seems to be the most promising measure, with the advantage that relative measurements suffer less from practice variations compared to absolute (changes in) values.

The reviewed studies yield variable results in the absolute values and in both the sign and magnitude of the relative changes of $A D C$ values which may be explained by the used techniques. Diffusion values measured with $D W I$ and subsequent $A D C$ mapping depend on the pulse sequence and post-processing methods used. With ADC mapping, typically one diffusivity value is assigned to all water present inside a voxel, whereas it is known that the microstructure of tissue is reflected in different diffusivity values for water molecules inside different compartments of the tissue ${ }^{59}$. For example, the diffusivity of intracellular water, extracellular water and that of water in the blood inside blood vessels will be different. On top of that, diffusion inside tissue may be anisotropic and randomly oriented capillary blood flow inside a voxel may influence the diffusion values measured. The influence of these effects on the measured diffusion values depends on sequence parameter settings, like the choice of b-values ${ }^{60,61}$. Therefore, the diffusivity derived from DWI at multiple $b$-values is called the apparent diffusion coefficient. For the included studies the chosen lowest $b$-value was $b=0$ in most studies, and $b=50$ in two studies ${ }^{45-58}$. The chosen highest $b$-value varied between $b=600$ and $b=1000$ among all included studies.

In addition, DWI scans are typically based on sequences employing an echo planar imaging (EPI) read-out. Such scans are prone to artefacts caused by field inhomogeneities, and to chemical shift artefacts caused by the presence of water and fat signals resonating at different frequencies ${ }^{62}$. With improper fat suppression, $A D C$ values measured may be corrupted by the presence of fat. Another important cause of variation in ADC measurements is the ROI size and placement. Most studies, but not all, delineated the ROI on DWI (guided by anatomical sequences) $45,47,49-58$. This is regarded the most optimal method because of the geometrical distortion that may occur on EPI read-out based DWl ${ }^{62}$. A single ROI, multiple ROls or volume of interest (VOI) were outlined on the DWI before start of the treatment and most studies adjusted the ROI(s)/VOI on the follow-up DWI ${ }^{46-}$ 49,51-53,55,57,58. Also, some studies calculated mean ADC values whereas other reported median ADC values (or percentiles) which complicated the comparison between the studies.

Furthermore, in case of a good/complete response to treatment, studies generally did not outline how the investigators dealt with disappearance of the signal on DWI $16-51,55$. Only three studies stated that the ROI was delineated according to the apparent tumour bed ${ }^{52,53,57}$. The optimal placement of the ROI and how to deal with shrinkage of the oesophageal tumour, especially in case of a good or 
even complete response to treatment, is still unknown. Theoretically, with an adjusted ROI per time point, the intrinsic signal within the residual suspect area is measured specifically, but the correct placement of the ROI in the most suspect areas is crucial in that case. Interobserver variability then becomes an issue. ROls that over time include the complete original tumour area may also enable measurement of changes in the tissue that responded to the treatment, but this delineation is difficult due to shrinkage of the tumour and thereby thinning of the oesophageal wall.

Lastly, next to the above discussed variation in b-values and ROI placement, a potential technical explanation for variation in ADC measurements is the use of different field strengths: both $1.5 \mathrm{~T}$ and 3.0 T MR scanners were used. In this systematic review we observed no consistent differences between studies on 3.0 T versus 1.5 T MRI. Although a higher field strength has the advantage of an increased intrinsic signal-to-noise ratio, the diffusion-weighted imaging may suffer from increased magnetic susceptibility artefacts at air-tissue interfaces. For all the aforementioned technical reasons, efforts should be made to standardize DWI sequences and the methods used to calculate the ADC in order to achieve comparable results in MRI of the oesophagus.

The varying results of the included studies may also be explained by the intrinsic tumour characteristics. For example, ACs and SCCs have different response rates to chemoradiotherapy ${ }^{2}$. However, no specific differences in ADC values were observed between AC and SCC. In SCC tumours the pre-treatment $A D C$ varied from 1.27 to 2.24 in complete responders and from 1.09 to 1.83 in PR/SD/PD patients $53,54,56-58$. In studies including (mainly) AC pre-treatment ADC values varied from 1.17 to 2.22 in good responders and 1.03-2.62 in poor responders ${ }^{48,55}$. Also, a recent study on $A C$ subtypes (according to the Laurén classification) showed that TRG 1/2 was found more often in intestinal $(60 \%)$ compared to diffuse subtype oesophageal AC $(24 \%)^{63}$. Furthermore, in rectal cancer MRI studies mucinous tumours are commonly excluded from analyses because they have high ADC values due to their low cellular density which introduces bias into the results ${ }^{64-66}$. The Laurén classification or mucinous tumour characteristics were unreported in the included studies, thus these intrinsic tumour effects could not be evaluated.

Although not all studies reached significance, the relative $A D C$ increase after two weeks of treatment showed a trend towards a larger increase of $A D C$ in good responders compared to poor responders $47-49,51,52,55,57,58$. This is probably due to the early effect of (chemo-)radiotherapy on the tumour microenvironment inducing tumour necrosis, with a loss of cell membrane integrity and an increase of the extracellular space, typically resulting in an increase of the ADC. However, the magnitude of the relative $A D C$ increase during treatment and thereby the cut-off between complete and incomplete responders differed per study (14-29\%). These differences in relative $A D C$ increase may be (partially) explained by the definition of a complete response, since some studies not only discriminate complete responders but also include near-complete responders in the good responder group. Furthermore, the studies in patients undergoing definitive chemo- and/ or radiotherapy did not have histopathology as the reference standard but instead used RECIST criteria (or endoscopic assessment in one case ${ }^{50}$ ). RECIST criteria are difficult to use in oesophageal 
tumours as following RECIST guidelines tumour diameters can only be measured in the axial planes, while oesophageal tumours mostly grow longitudinally along the oesophageal wall, which can be better measured in the sagittal or coronal plane ${ }^{23,67}$. Also, wall thickening often remains present after chemoradiotherapy due to radiation esophagitis, even in the absence of residual tumour.

Results on the relative increase in ADC after treatment (\% $\mathrm{ADCpost}$ ) instead of during treatment varied more substantially between the studies, which may only for a small part be explained by differences regarding the definition of complete response. Notably, the time-point of ADCpost measurements varied from a few days up to 9 weeks after treatment. The presence of radiation induced inflammation effects shortly after completion of (chemo-)radiotherapy is likely to influence ADC measurements early after treatment. Also, as stated before, $\mathrm{ROI}$ size and placement for ADC measurements after treatment is hampered by small or absent tumour after treatment in case of a good response. Therefore, measurements of ADC for quantitative analyses may be useful before and during CRT, whereas response after CRT may likely rather be assessed visually as was shown in a recent study ${ }^{44}$. However, the addition of endoscopy, EUS and FDG-PET/CT to the visual MRI assessment may be important in order to select complete responders for organ preservation.

In general, the studies included in this systematic review suggest that ADC measurements on DW-MRI have potential for prediction of response to treatment in oesophageal cancer patients, especially the relative increase in ADC two weeks after the onset of treatment seems promising. However, specific ADC cut-off values considerably differed between studies and therefore cannot (yet) be applied in clinical practice on individual patients. Efforts should be made to standardize DWI sequences and the methods used to calculate the $A D C$ in order to achieve comparable results. 


\section{REFERENCES}

1. American Cancer Society. Cancer facts \& figures. Atlanta: American Cancer Society; 2016. p. 18.

2. van Hagen $P$, Hulshof $M C$, van Lanschot JJ, Steyerberg EW, van Berge Henegouwen MI, Wijnhoven BP, et al. Preoperative chemoradiotherapy for esophageal or junctional cancer. N Engl J Med 2012;366:2074-84.

3. Shapiro J, van Lanschot JJB, Hulshof $M$, van Hagen $P$, van Berge Henegouwen MI, Wijnhoven $B P L$, et al. Neoadjuvant chemoradiotherapy plus surgery versus surgery alone for oesophageal or junctional cancer (CROSS): long-term results of a randomised controlled trial. Lancet Oncol 2015;16:1090-8.

4. Chiappa A, Andreoni B, Dionigi R, Spaggiari $L$, Foschi D, Polvani $G$, et al. A rationale multidisciplinary approach for treatment of esophageal and gastroesophageal junction cancer: Accurate review of management and perspectives. Crit Rev Oncol Hematol 2018;132:161-8.

5. Mandard AM, Dalibard F, Mandard JC, Marnay J, Henry-Amar M, Petiot JF, et al. Pathologic assessment of tumor regression after preoperative chemoradiotherapy of esophageal carcinoma. Clinicopathologic correlations. Cancer 1994;73:2680-6.

6. Zhang $Z$, Zhang $H$. Impact of neoadjuvant chemotherapy and chemoradiotherapy on postoperative cardiopulmonary complications in patients with esophageal cancer. Dis Esophagus 2017;30:1-7.

7. Wieder HA, Ott K, Lordick F, Becker K, Stahl A, Herrmann K, et al. Prediction of tumor response by FDG-PET: comparison of the accuracy of single and sequential studies in patients with adenocarcinomas of the esophagogastric junction. Eur J Nucl Med Mol Imaging 2007;34:1925-32.

8. Cuenca $X$, Hennequin C, Hindie E, Rivera $\mathrm{S}$, Vercellino L, Baruch-Hennequin V, et al. Evaluation of early response to concomitant chemoradiotherapy by interim 18F FDG PET/ CT imaging in patients with locally advanced oesophageal carcinomas. Eur J Nucl Med Mol Imaging 2013;40:477-85.

9. Gillham CM, Lucey JA, Keogan M, Duffy GJ, Malik V, Raouf AA, et al. (18)FDG uptake during induction chemoradiation for oesophageal cancer fails to predict histomorphological tumour response. Br J Cancer 2006;95:1174-9.

10. Malik V, Lucey JA, Duffy GJ, Wilson L, McNamara L, Keogan M, et al. Early repeated 18F-FDG PET scans during neoadjuvant chemoradiation fail to predict histopathologic response or survival benefit in adenocarcinoma of the esophagus. J Nucl Med 2010;51:1863-9.

11. van Heij $M$, Omloo JM, van Berge Henegouwen MI, Hoekstra OS, Boellaard R, Bossuyt PM, et al. Fluorodeoxyglucose positron emission tomography for evaluating early response during neoadjuvant chemoradiotherapy in patients with potentially curable esophageal cancer. Ann Surg $2011 ; 253: 56-63$.

12. Butof R, Hofheinz F, Zophel K, Stadelmann T, Schmollack J, Jentsch C, et al. Prognostic value of pretherapeutic tumor-to-blood standardized uptake ratio in patients with esophageal carcinoma. J Nucl Med 2015;56:1150-6.

13. Castoro C, Scarpa M, Cagol M, Alfieri R, Ruol A, Cavallin $F$, et al. Complete clinical response after neoadjuvant chemoradiotherapy for squamous cell cancer of the thoracic oesophagus: is surgery always necessary? J Gastrointest Surg 2013;17:1375-81.

14. Taketa T, Xiao L, Sudo K, Suzuki A, Wadhwa $R$, Blum MA, et al. Propensitybased matching between esophagogastric cancer patients who had surgery and who declined surgery after preoperative chemoradiation. Oncology 2013;85:95-9.

15. Furlong $H$, Bass $G$, Breathnach $O, O^{\prime}$ Neill $B$, Leen E, Walsh TN. Targeting therapy for esophageal cancer in patients aged 70 and over. J Geriatr Oncol 2013;4:107-13.

16. Noordman BJ, Wijnhoven BPL, Lagarde SM, Boonstra JJ, Coene P, Dekker JWT, et al. Neoadjuvant chemoradiotherapy plus surgery versus active surveillance for oesophageal cancer: a stepped-wedge cluster randomised trial. BMC Cancer 2018;18:142.

17. Ohkura Y, Shindoh J, Ueno M, lizuka T, Udagawa $H$. Comparison of outcome of esophagectomy versus nonsurgical treatment for resectable esophageal cancer with clinical complete response to neoadjuvant therapy. Ann 
Surg Oncol 2018;25:2428-33.

18. Yip C, Cook GJ, Landau DB, Davies A, Goh V. Performance of different imaging modalities in assessment of response to neoadjuvant therapy in primary esophageal cancer. Dis Esophagus 2016;29:116-30.

19. Goense L, van Rossum PS, Reitsma JB, Lam $M G$, Meijer GJ, van Vulpen $M$, et al. Diagnostic performance of (1)(8)F-FDG PET and PET/ CT for the detection of recurrent esophageal cancer after treatment with curative intent: a systematic review and meta-analysis. J Nucl Med 2015;56:995-1002.

20. Koh DM, Collins DJ. Diffusion-weighted MRI in the body: applications and challenges in oncology. AJR Am J Roentgenol 2007;188:1622-35.

21. van Rossum PS, van Hillegersberg $R$, Lever $F M$, Lips IM, van Lier AL, Meijer GJ, et al. Imaging strategies in the management of oesophageal cancer: what's the role of MRI? Eur Radiol 2013;23:1753-65.

22. Duffaud F, Therasse $P$. New guidelines to evaluate the response to treatment in solid tumors. Bull Cancer 2000;87:881-6.

23. Eisenhaver EA, Therasse $P$, Bogaerts J, Schwartz $L H$, Sargent D, Ford R, et al. New response evaluation criteria in solid fumours: revised RECIST guideline (version 1.1). Eur J Cancer 2009;45:228-47.

24. Moher D, Liberati A, Tetzlaff J, Altman DG, Group P. Preferred reporting items for systematic reviews and meta-analyses: the PRISMA statement. BMJ (Clin Res Ed) 2009;339:b2535.

25. Whiting PF, Rutjes AW, Westwood ME, Mallett S, Deeks JJ, Reitsma JB, et al. QUADAS-2: a revised tool for the quality assessment of diagnostic accuracy studies. Ann Intern Med 2011;155:529-36.

26. Sakurada A, Takahara T, Kwee TC, Yamashita T, Nasu $S$, Horie T, et al. Diagnostic performance of diffusion-weighted magnetic resonance imaging in esophageal cancer. Eur Radiol 2009;19:1461-9.

27. Aoyagi T, Shuto K, Okazumi S, Hayano K, Satoh $\mathrm{A}$, Saitoh $\mathrm{H}$, et al. Apparent diffusion coefficient correlation with oesophageal tumour stroma and angiogenesis. Eur Radiol 2012;22:1172-7.

28. Lee G, Han I, Kim SJ, Jeong YJ, Kim IJ, Pak
$\mathrm{K}$, et al. Clinical implication of PET/MR imaging in preoperative esophageal cancer staging: comparison with $\mathrm{PET} / \mathrm{CT}$, endoscopic ultrasonography, and CT. J Nucl Med 2014;55:1242-7.

29. Malik V, Harmon M, Johnston C, Fagan AJ, Claxton Z, Ravi N, et al. Whole body MRI in the staging of esophageal cancer-a prospective comparison with whole body 18F-FDG PET-CT. Dig Surg 2015;32:397-408.

30. Sun YS, Cui Y, Tang L, Qi LP, Wang N, Zhang $X Y$, et al. Early evaluation of cancer response by a new functional biomarker: apparent diffusion coefficient. AJR Am J Roentgenol 2011;197:W23-9.

31. Wang L, Han C, Zhu S, Shi G, Wang Q, Tian $\mathrm{H}$, et al. Investigation of using diffusion-weighted magnetic resonance imaging to evaluate the therapeutic effect of esophageal carcinoma treatment. Oncol Res Treat 2014;37:112-6.

32. Giganti F, Ambrosi A, Petrone MC, Canevari C, Chiari D, Salerno A, et al. Prospective comparison of $M R$ with diffusion-weighted imaging, endoscopic ultrasound, MDCT and positron emission tomography-CT in the pre-operative staging of oesophageal cancer: results from a pilot study. Br J Radiol 2016;89:20160087.

33. Chang EY, Li X, Jerosch-Herold M, Priest RA, Enestvedt $C K, X \cup J$, et al. The evaluation of esophageal adenocarcinoma using dynamic contrast-enhanced magnetic resonance imaging. J Gastrointest Surg 2008;12:166-75.

34. Lei J, Tian Y, Zhu S-C, Han Q, Wei Y, Yang S, et al. Preliminary study of IVIMDWI and DCE-MRI in early diagnosis of esophageal cancer. Eur Rev Med Pharmacol Sci 2015;19:3345-50.

35. van Rossum PS, van Lier $A L$, van Vulpen $M$, Reerink O, Lagendijk JJ, Lin SH, et al. Diffusionweighted magnetic resonance imaging for the prediction of pathologic response to neoadjuvant chemoradiotherapy in esophageal cancer. Radiother Oncol 2015;115:163-70.

36. Zhu S, Wei Y, Gao F, Li L, Liu Y, Huang Z, ef al. Esophageal carcinoma: intravoxel incoherent motion diffusion-weighted MRI parameters and histopathological correlations. J Magn Reson Imaging 2018.

37. Giganti F, Salerno A, Ambrosi A, Chiari D, Orsenigo E, Esposito A, et al. Prognostic utility of diffusion-weighted MRI in oesophageal cancer: 
is apparent diffusion coefficient a potential marker of tumour aggressiveness? Radiol Med 2016;121:173-80.

38. Goense L, Heethuis SE, van Rossum PSN, Voncken FEM, Lagendijk JJW, Lam M, et al. Correlation between functional imaging markers derived from diffusionweighted MRI and 18F-FDG PET/ $\mathrm{CT}$ in esophageal cancer. Nucl Med Commun 2018;39:60-7.

39. Ye Z, Fang J, Dai S, Xie T, Wang F, Wang Z, et al. Inter- and intra-observer reproducibility of ADC measurements in esophageal carcinoma primary tumors. Oncotarget 2017;8:92880-9.

40. Giganti F, Orsenigo E, Esposito A, Chiari D, Salerno A, Ambrosi A, et al. Prognostic role of diffusion-weighted MR imaging for resectable gastric cancer. Radiology 2015;276:444-52.

41. Qiu B, Wang D, Yang H, Xie W, Liang Y, Cai P, et al. Combined modalities of magnetic resonance imaging, endoscopy and computed tomography in the evaluation of tumor responses to definitive chemoradiotherapy in esophageal squamous cell carcinoma. Radiother Oncol 2016;121:239-45.

42. Liu G, Yang Z, Li T, Yang L, Zheng X, Cai L. Optimization of $b$-values in diffusionweighted imaging for esophageal cancer: Measuring the longitudinal length of gross tumor volume and evaluating chemoradiotherapeutic efficacy. J Cancer Res Ther 2017;13:748-55.

43. Belmouhand $M$, Lofgren J, Johannesen $H H$, Baeksgaard L, Gutte H, Tariq K, et al. Early response evaluation of neoadjuvant therapy with $\mathrm{PET} / \mathrm{MRI}$ to predict resectability in patients with adenocarcinoma of the esophagogastric junction. Abdom Radiol 2018.

44. Vollenbrock SE, Voncken FEM, van Dieren JM, Lambregts DMJ, Maas M, Meijer GJ, et al. Diagnostic performance of MRI for assessment of response to neoadjuvant chemoradiotherapy in oesophageal cancer. Br J Surg 2019;106:596605.

45. Aoyagi T, Shuto K, Okazumi S, Shimada H, Kazama T, Matsubara H. Apparent diffusion coefficient values measured by diffusionweighted imaging predict chemoradiotherapeutic effect for advanced esophageal cancer. Dig Surg $2011 ; 28: 252-7$.

46. De Cobelli F, Giganti F, Orsenigo E, Cellina M, Esposito A, Agostini G, et al. Apparent diffusion coefficient modifications in assessing gastro- oesophageal cancer response to neoadjuvant treatment: comparison with tumour regression grade at histology. Eur Radiol 2013;23:216574.

47. Imanishi S, Shuto K, Aoyagi T, Kono T, Saito $\mathrm{H}$, Matsubara H. Diffusionweighted magnetic resonance imaging for predicting and detecting the early response to chemoradiotherapy of advanced esophageal squamous cell carcinoma. Dig Surg 2013;30:240-8.

48. Weber MA, Bender K, von Gall CC, Stange A, Grunberg K, Ott K, et al. Assessment of diffusionweighted MRI and 18F-fluoro-deoxyglucose PET/ $\mathrm{CT}$ in monitoring early response to neoadjuvant chemotherapy in adenocarcinoma of the esophagogastric junction. J Gastrointest Liver Dis 2013;22:45-52.

49. Kwee RM, Dik AK, Sosef MN, Berendsen RC, Sassen S, Lammering $G$, et al. Interobserver reproducibility of diffusion-weighted MRI in monitoring tumor response to neoadjuvant therapy in esophageal cancer. PLoS ONE 2014;9: e92211.

50. Guo L, Zhang L, Zhao J. CT scan and magnetic resonance diffusion-weighted imaging in the diagnosis and treatment of esophageal cancer. Oncol Lett 2018;16:7117-22.

51. Heethuis SE, Goense L, van Rossum PSN, Borggreve AS, Mook S, Voncken FEM, et al. DWMRI and DCE-MRI are of complementary value in predicting pathologic response to neoadjuvant chemoradiotherapy for esophageal cancer. Acta Oncol 2018;57:1201-8.

52. Li FP, Wang $\mathrm{H}$, Hou J, Tang J, Lu Q, Wang LL, et al. Utility of intravoxel incoherent motion diffusion-weighted imaging in predicting early response to concurrent chemoradiotherapy in oesophageal squamous cell carcinoma. Clin Radiol 2018;73(756):e17-26.

53. Li QW, Qiu B, Wang B, Wang DL, Yin SH, Yang $H$, et al. Prediction of pathologic responders to neoadjuvant chemoradiotherapy by diffusionweighted magnetic resonance imaging in locally advanced esophageal squamous cell carcinoma: a prospective study. Dis Esophagus 2018;31:1090.

54. Liu S, Zhen F-X, Sun N, Chen J, Cao Y, Zhang $\mathrm{S}$, et al. Apparent diffusion coefficient values detected by diffusion-weighted imaging in the prognosis of patients with locally advanced esophageal squamous cell carcinoma 
receiving chemoradiation. OncoTarget Ther 2016;9:5791-6.

55. Fang $P$, Musall BC, Son JB et al. Multimodal Imaging of Pathologic Response to Chemoradiation in Esophageal Cancer. Int J Radiat Oncol Biol Phys 2018;102:996-1001.

56. Kozumi M, Ota $H$, Yamamoto $T$, Umezawa $R$, Matsushita $\mathrm{H}$, Ishikawa $Y$, et al. Oesophageal squamous cell carcinoma: histogram-derived $A D C$ parameters are not predictive of tumour response to chemoradiotherapy. Eur Radiol 2018;28:4296-305.

57. Zheng $H$, Ren $W$, Pan $X$, Zhang Q, Liu B, Liu $S$, et al. Role of intravoxel incoherent motion MRI in early assessment of the response of esophageal squamous cell carcinoma to chemoradiotherapy: a pilot study. J Magn Reson Imaging 2018;48:349-58.

58. Wang L, Liu L, Han C, Liu S, Tian H, Li Z, et al. The diffusion-weighted magnetic resonance imaging (DWI) predicts the early response of esophageal squamous cell carcinoma to concurrent chemoradiotherapy. Radiother Oncol 2016;121:246-51.

59. Le Bihan D. Apparent diffusion coefficient and beyond: what diffusion MR imaging can tell us about tissue structure. Radiology 2013;268:31822.

60. Le Bihan D, Breton E, Lallemand D, Aubin ML, Vignaud J, Laval-Jeantet M. Separation of diffusion and perfusion in intravoxel incoherent motion MR imaging. Radiology 1988; 168:497505.

61. 61. Thormer G, Otto J, Reiss-Zimmermann $M$, Seiwerts M, Moche M, Garnov N, et al. Diagnostic value of $A D C$ in patients with prostate cancer: influence of the choice of $b$ values. Eur Radiol 2012;22:1820-8.

62. Le Bihan D, Poupon C, Amadon A, Lethimonnier F. Artifacts and pitfalls in diffusion MRI. J Magn Reson Imaging 2006;24:478-88.

63. van der Kaaij RT, Snaebjornsson P, Voncken $F E$, van Dieren JM, Jansen EP, Sikorska K, et al. The prognostic and potentially predictive value of the Lauren classification in oesophageal adenocarcinoma. Eur J Cancer 2017;76:27-35.

64. Curvo-Semedo L, Lambregts DM, Maas M, Beets $\mathrm{GL}$, Caseiro-Alves F, Beets-Tan RG. Diffusionweighted MRI in rectal cancer: apparent diffusion coefficient as a potential noninvasive marker of tumor aggressiveness. J Magn Reson Imaging 2012;35:1365-71.

65. Colakoglu Er H, Erden A. Mean ADC values discriminate rectal mucinous carcinoma from rectal nonmucinous adenocarcinoma. Turk J Med Sci 2017;47:1520-5.

66. van Heeswijk MM, Lambregts DMJ, Maas $M$, Lahaye MJ, Ayas Z, Slenter J, et al. Measuring the apparent diffusion coefficient in primary rectal tumors: is there a benefit in performing histogram analyses? Abdom Radiol 2017;42:1627-36.

67. Therasse P, Arbuck SG, Eisenhauer EA, Wanders $J$, Kaplan RS, Rubinstein L, et al. New guidelines to evaluate the response to treatment in solid tumors. European Organization for Research and Treatment of Cancer, National Cancer Institute of the United States, National Cancer Institute of Canada. J Natl Cancer Inst 2000;92:205-16.

68. Becker K, Mueller JD, Schulmacher C, Ott K, Fink U, Busch R, et al. Histomorphology and grading of regression in gastric carcinoma treated with neoadjuvant chemotherapy. Cancer 2003;98:1521-30. 


\section{SUPPLEMENTARY FILES}

Suppl. Table 1. Literature search performed on February 27th, 2019

\begin{tabular}{lc}
\hline Search term & Results \\
\hline 1. exp esophageal cancer/ & 61776 \\
\hline 2. exp oesophagus/ & 66401 \\
\hline 3. (oesphag* or esophag*).ti,ab. & 354558 \\
\hline 4. exp cancer/ & 6287300 \\
\hline $\begin{array}{l}\text { 5. (neoplas* or tumor* or tumor* or cancer* or malignan* or } \\
\text { oncolog* or carcinoma*).ti,ab. }\end{array}$ & 8119204 \\
\hline 6. 4 or 5 & 9459032 \\
\hline 7. 2 or 3 & 376529 \\
\hline 8. 6 and 7 & 166796 \\
\hline 9. 1 or 8 & 183006 \\
\hline 10. exp MRI/ & 853731 \\
\hline 11. (diffusion weighted imaging or nuclear magnetic resonance \\
imaging or mri or magnetic resonance imaging or diffusion MRI & 1015992 \\
or DW MRI or diffusion weighted imaging or ADC or NMR imag* \\
or DCE or dynamic contrast enhanced).ti,ab. \\
\hline 12. 10 or 11 & \\
\hline 13. 9 and 12 & 1411359 \\
\hline 14. exp treatment response/ & 3273 \\
\hline 15. (predict* or pronos* or respons* or diagnos*).ti,ab. & 230455 \\
\hline 16. 14 or 15 & 16035453 \\
\hline 17. 13 and 16 & 16119676 \\
\hline 18. limit 17 to (dutch or english) & 1991 \\
\hline 19. limit 18 to yr="2000 -Current" & 1764 \\
\hline 20. remove duplicates from 19 & 1659 \\
\hline
\end{tabular}
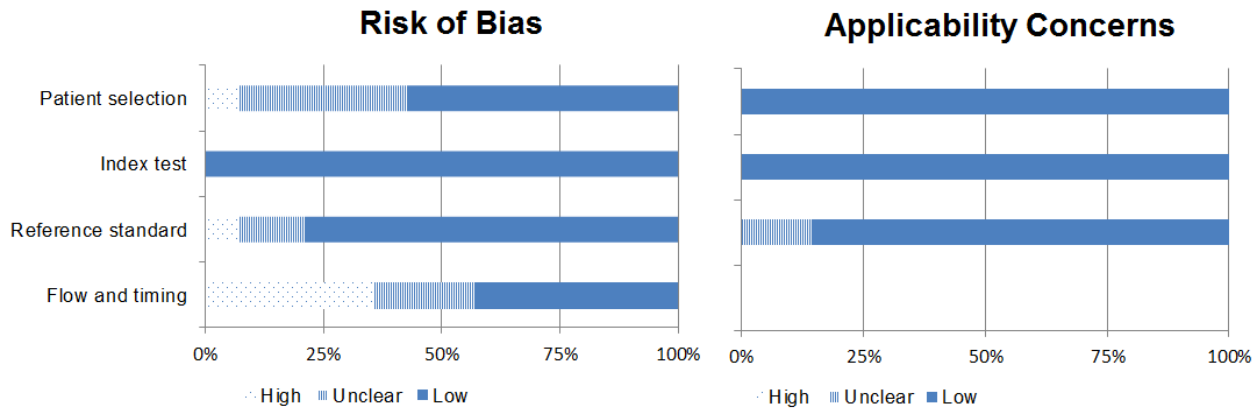

Suppl. Figure 1. Quality of the included studies evaluated according to the Quality Assessment of Diagnostic Accuracy Studies-2 (QUADAS-2) criteria ${ }^{25}$ 


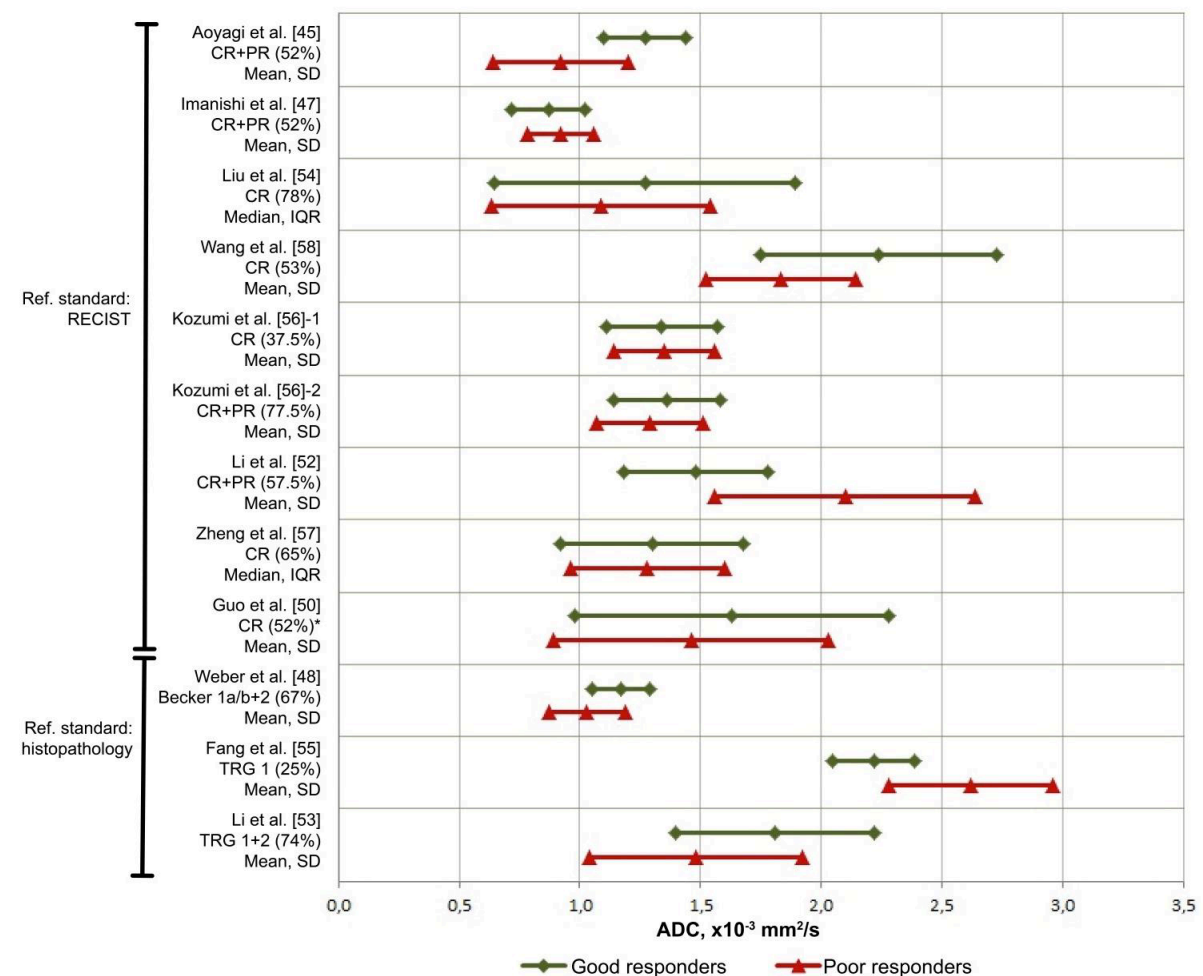

Suppl. Figure 2. Response prediction before treatment: absolute ADC values for good responders versus poor responders.

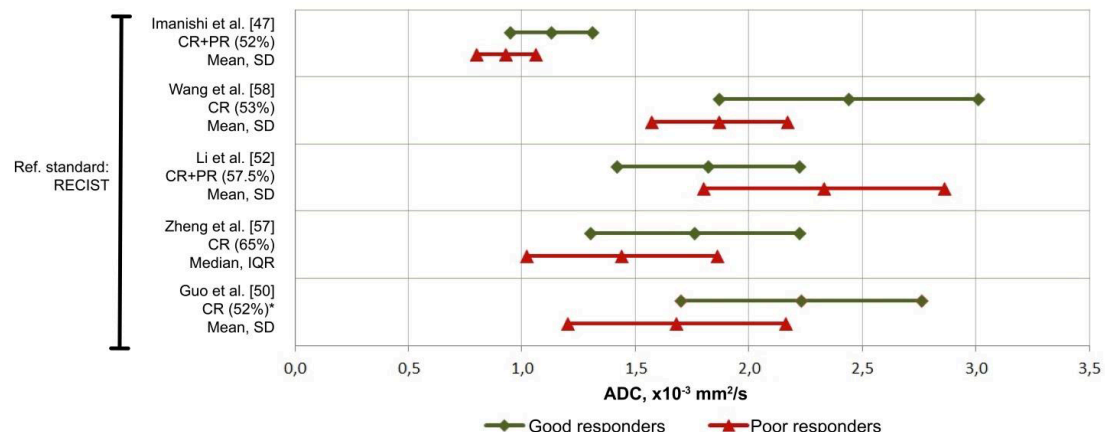

Suppl. Figure 3. Response prediction during treatment: absolute ADC values

Abbreviations of suppl. Fig 2 \& 3: RECIST = Response Evaluation Criteria In Solid Tumours ${ }^{23}$; $A D C=$ apparent diffusion coefficient; $C R=$ complete response according to RECIST; PR = partial response according to RECIST; Becker = histology according to the Becker score ${ }^{68}$; TRG = tumour regression grade according to Mandard $^{5}$; IQR = interquartile range; SD = standard deviation. The names represent first authors of included studies, followed by the definition of a good responder and the proportion of good responders in the particular study.

* This study used clinical complete response on endoscopy as the reference standard. 


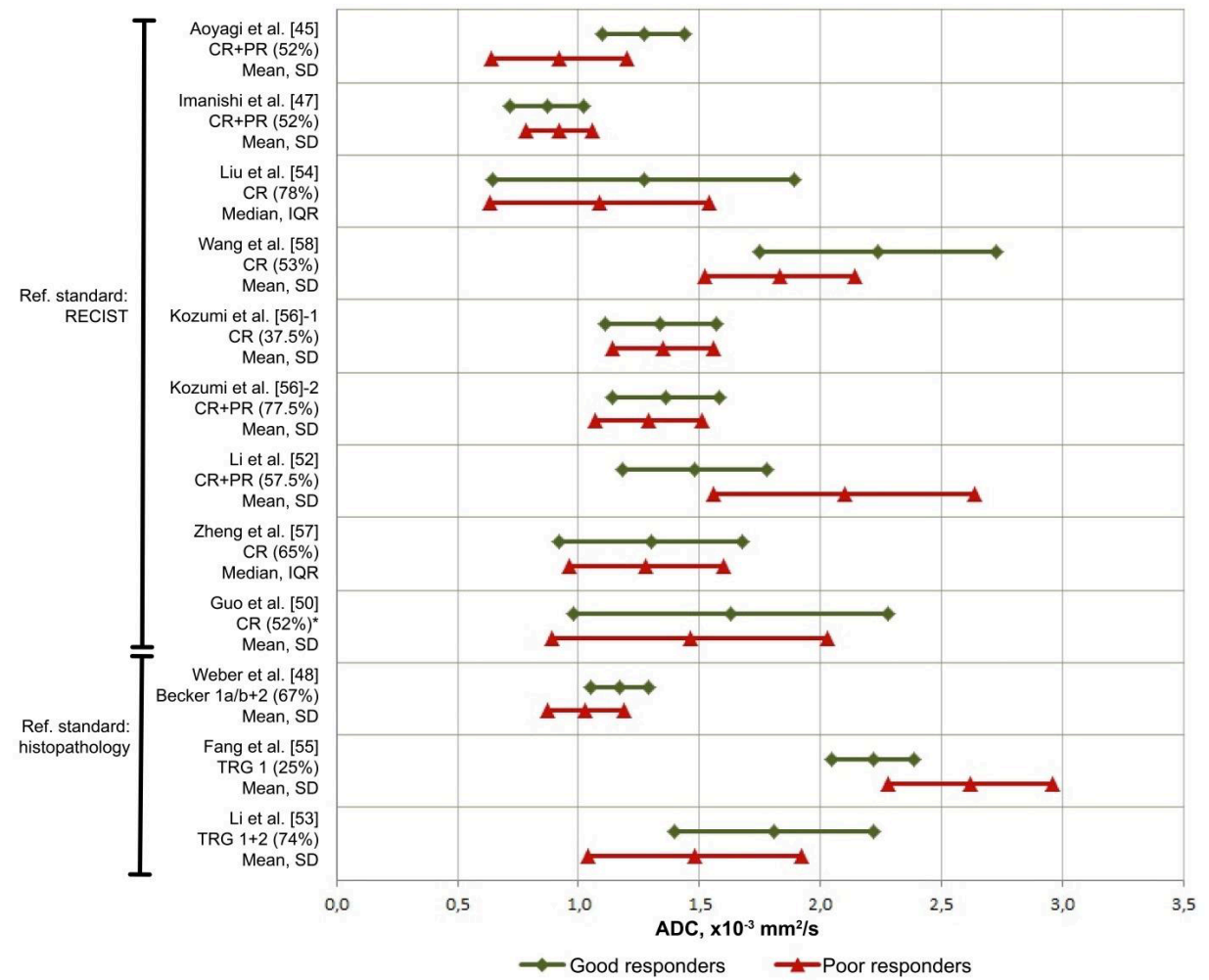

Suppl. Figure 4. Response assessment after treatment: absolute ADC values

Abbreviations: RECIST = Response Evaluation Criteria In Solid Tumors ${ }^{23}$; ADC $=$ apparent diffusion coefficient; $\mathrm{CR}=$ complete response according to $\mathrm{RECIST}$; $\mathrm{PR}=$ partial response according to RECIST; TRG = tumor regression grade according to Mandard5; IQR = interquartile range; $\mathrm{SD}=$ standard deviation.

The names represent first authors of included studies, followed by the definition of a good responder and the proportion of good responders in the particular study.

* This study measured ADC on DW-MRI directly after completion of treatment (Zheng-1) and at one month after completion of treatment (Zheng-2).

** This study used clinical complete response on endoscopy as the reference standard. 


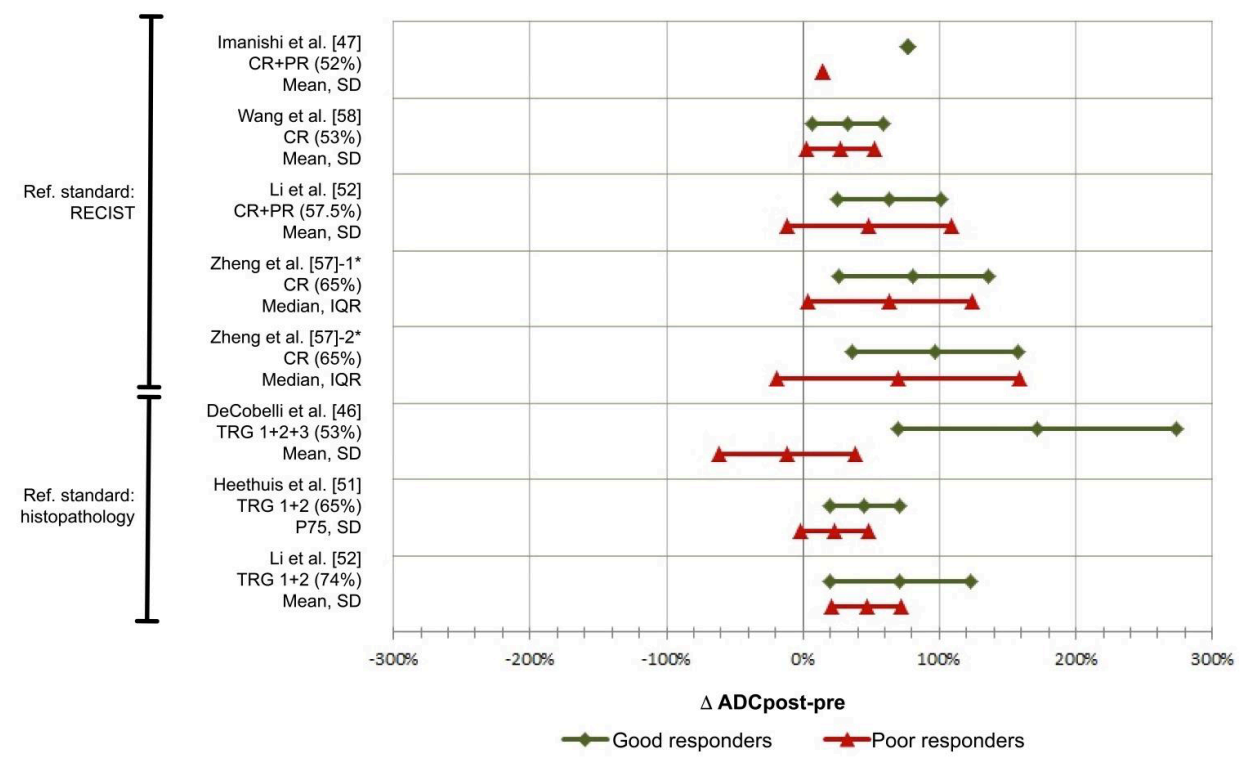

Suppl. Figure 5. Relative difference in $A D C$ after treatment (\% $\triangle A D C$ post-pre) for responders versus poor responders

Abbreviations: RECIST = Response Evaluation Criteria In Solid Tumors ${ }^{23}$; ADC = apparent diffusion coefficient; $\mathrm{CR}$ = complete response according to $\mathrm{RECIST} ; \mathrm{PR}=$ partial response according to $\mathrm{RECIST}$; TRG = tumor regression grade according to Mandard $; \mathrm{IQR}=$ interquartile range; $\mathrm{SD}=$ standard deviation.

The names represent first authors of included studies, followed by the definition of a good responder and the proportion of good responders in the particular study.

* This study measured ADC on DW-MRI directly after completion of treatment (Zheng-1) and at one month after completion of treatment (Zheng-2). 
DIFFUSION-WEIGHTED MRI WITH ADC MAPPING FOR RESPONSE PREDICTION: A SYSTEMATIC REVIEW 


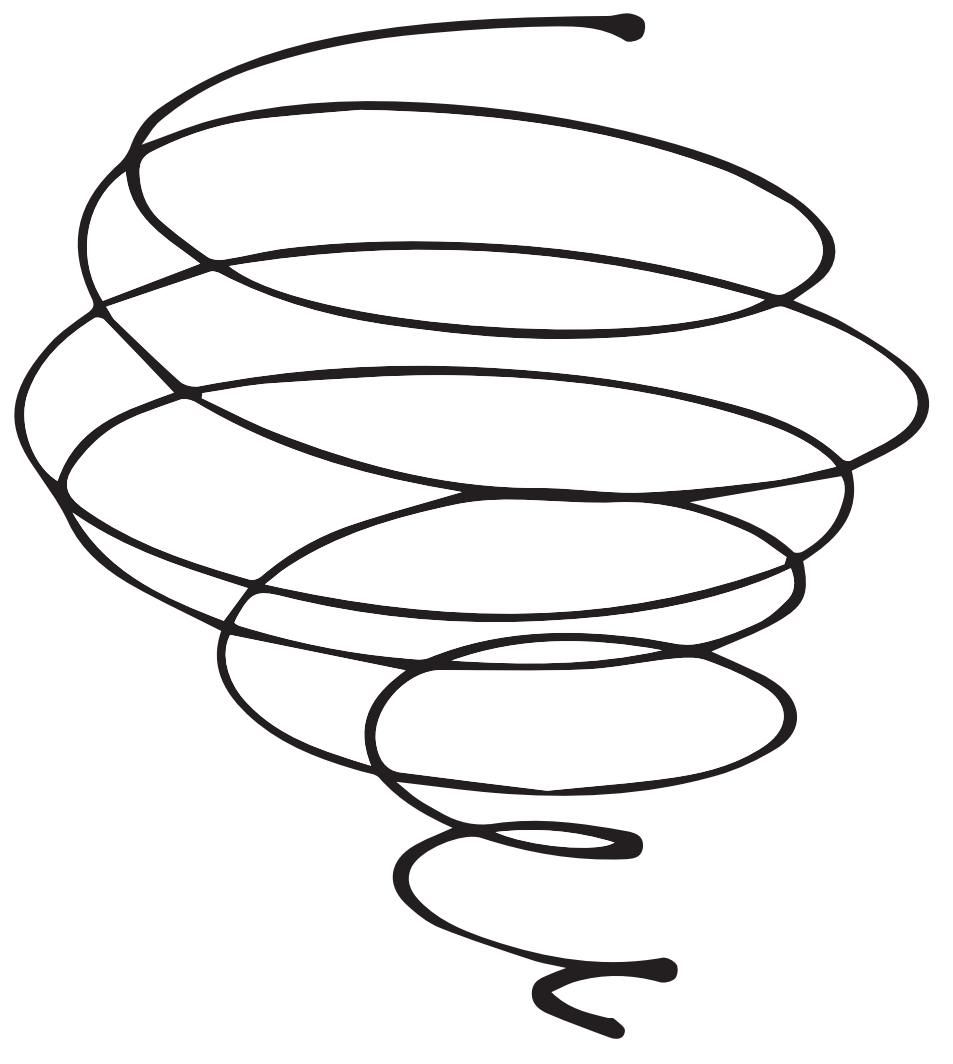




\section{CHAPTER 4}

\section{Clinical response assessment on DW-MRI compared with FDG-PET/CT after neoadjuvant chemoradiotherapy in patients with oesophageal cancer}

European Journal of Nuclear Medicine and Molecular Imaging, Jun 2020 (Online, ahead of print)

Sophie E. Vollenbrock

Francine E.M. Voncken

Doenja M.J. Lambregts

Monique Maas

Maarten L. Donswijk

Erik Vegt

Leon C. ter Beek

Jolanda M. van Dieren

Johanna W. van Sandick

Berthe M.P. Aleman

Regina G.H. Beets-Tan

Annemarieke Bartels-Rutten 


\begin{abstract}
Purpose

In about $30 \%$ of patients treated with neoadjuvant chemoradiotherapy (nCRT) followed by surgical resection for locally advanced oesophageal cancer no vital tumour is found in the resection specimen. Accurate clinical response assessment is critical if deferral from surgery is considered in complete responders. Our study aimed to compare the performance of MRI and of FDG-PET/CT for the detection of residual disease after nCRT.
\end{abstract}

Methods

Patients with oesophageal cancer eligible for nCRT and oesophagectomy were prospectively included. All patients underwent FDG-PET/CT and MRI before and between 6 and 8 weeks after nCRT. Two radiologists scored the MRI scans, and two nuclear medicine physicians scored the FDGPET/CT scans using a 5-point score for residual disease. Histopathology after oesophagectomy represented the reference standard. Sensitivity, specificity, and area under the receiver operating characteristic curve (AUC) were calculated for detection of residual tumour (ypT+), residual nodal disease (ypN+), and any residual disease (ypT+Nx/ypTON+).

\title{
Results
}

Seven out of $33(21 \%)$ patients had a pathological complete response. The AUCs for individual readers to detect ypT+ were $0.71 / 0.70$ on diffusion-weighted (DW)-MRI and 0.54/0.57 on FDG-PET/CT, and to detect ypN+ were $0.89 / 0.81$ on DW-MRI and 0.75/0.71 on FDG-PET/CT. The AUCs/sensitivities/specificities for the individual readers to detect any residual disease were $0.74 / 92 \% / 57 \%$ and $0.70 / 96 \% / 43 \%$ on $M R I$; these were $0.49 / 69 \% / 29 \%$ and $0.60 / 69 \% / 43 \%$ on FDG-PET/CT, respectively.

\section{Conclusion}

MRI reached higher diagnostic accuracies than FDG-PET/CT for the detection of residual tumour in oesophageal cancer patients at 6 to 8 weeks after nCRT. 


\section{INTRODUCTION}

Oesophageal cancer is the ninth most common cancer globally'. Neoadjuvant chemo(radio) therapy is increasingly used, as it improves survival after surgery for locally advanced oesophageal cancer $^{2}$. In a large Dutch multicentre trial, a pathological complete response (pCR) was found in $29 \%$ of patients after neoadjuvant chemoradiotherapy $(\mathrm{nCRT})^{3}$. For clinical complete responders (cCR), the option of organ preservation after $\mathrm{nCRT}$ is currently being explored ${ }^{4,5}$. Accurate clinical response assessment is critical to select the right patients. So far, it is unclear what the optimal diagnostic modality or combination of modalities is.

18F-FDG is an analogue of glucose and is taken up into metabolically active cells by endothelial glucose transport. This causes FDG uptake on 18F-fluorodeoxyglucose positron emission tomography with computed tomography (FDG-PET/CT). This technique helps to detect interval distant metastases after $n C R T^{6,7}$. However, FDG-PET/CT is less accurate in detecting residual disease after chemoradiotherapy at the primary tumour site due to its inability to differentiate residual tumour and inflammation due to radiotherapy. Two recent meta-analyses reported pooled sensitivities of $73 \%$ and $74 \%$ and specificities of $62 \%$ and $52 \%$ for the detection of residual tumour after neoadjuvant treatment ${ }^{8,9}$.

Diffusion-weighted magnetic resonance imaging (DW-MRI) is superior in differentiating residual tumour from inflammation. Signal depends on the water diffusivity in tissues, which is restricted in tumours with a high cellular density ${ }^{10,11}$. In organ preservation treatment approaches for rectal cancer, DW-MRI has proven its additional value to endoscopy and clinical examination in the clinical response assessment after nCRT and has become part of the restaging work-up of patients considered for organ preservation. DW-MRI is especially accurate in detecting residual disease ${ }^{12,13}$. A preliminary study on DW-MRI for the assessment of response to $\mathrm{nCRT}$ in oesophageal cancer showed promising results with sensitivities of $90-97 \%$ for the detection of residual disease ${ }^{14}$. However, in contrast to rectal cancer diagnostic work-up, standard work-up in oesophageal cancer does not yet include MRI. While the inability to differentiate between residual tumour and radiation oesophagitis is a major drawback of FDG-PET/CT, DW-MRI could have added value in the response assessment of oesophageal cancer patients after chemoradiotherapy.

As yet, it is unknown how DW-MRI relates to FDG-PET/CT, as no prospective comparative studies have been performed. In the present study, the performance of DW-MRI was compared prospectively with that of FDG-PET/CT in detecting residual disease in patients with oesophageal cancer after nCRT. 


\section{METHODS}

\section{Patients}

The study population consisted of patients with biopsy-proven locally advanced, non-metastatic oesophageal cancer who were considered eligible for nCRT followed by oesophagectomy. Patients with general contraindications for MRI, for example, severe claustrophobia or a known metallic foreign body in the eye, were not eligible for inclusion. The study was approved by the local medical ethics committee (NCT 02139488), and written informed consent was obtained from all patients.

\section{Treatment}

All study patients underwent $\mathrm{nCRT}$ (total of $41.4 \mathrm{~Gy}$ in 23 fractions with weekly administration of carboplatin and paclitaxel) followed by oesophagectomy. Oesophagectomy was performed via the transhiatal or transthoracic approach depending on the location of the tumour and suspected or biopsy-proven nodal disease.

\section{Imaging}

According to local standard work-up, patients underwent FDG-PET/CT to rule out distant metastases before and after nCRT. For the purpose of the study, all patients underwent DW-MRI scans before and 6-8 weeks after nCRT. The post-nCRT DW-MRI and FDG-PET/CT scans were planned with a maximum time interval of 3 weeks between imaging and surgery.

\section{FDG-PET/CT acquisition}

The FDG-PET/CT scan after $n C R T$ was performed using the same system and settings in all patients. These scans were acquired using a dedicated FDG-PET/CT system (Gemini TF/Big Bore, Philips Medical Systems, Cleveland, Ohio, USA). Patients fasted for at least $6 \mathrm{~h}$ before the tracer injection ( $4 \mathrm{~h}$ in patients with diabetes mellitus). A PET scan was acquired at $2 \mathrm{~min}$ per bed position and was combined with a low-dose CT scan (dose modulated, $40 \mathrm{mAs}, 2-\mathrm{mm}$ slice thickness) for attenuation correction and anatomical correlation. The scan was performed from skull base to thighs and started $60 \pm 10$ min after the administration of 18F-FDG (190-260 MBq). Images were reconstructed using iterative 3D reconstruction. In most patients (61\%), the baseline FDG-PET/CT scan was performed in The Netherlands Cancer Institute, whereas some patients underwent the pre-treatment FDG-PET/CT scan in a referring hospital following standard protocols for clinical staging according to the EANM procedure guidelines for tumour imaging ${ }^{15}$.

\section{MRI acquisition}

MR imaging (at baseline and 6-8 weeks after nCRT) was performed on a 1.5-Tesla MRI scanner (Philips Achieva, Best, The Netherlands), using Torso-XL (16 channel) receiver coils. The MRI 
consisted of T2-weighted multi-slice turbo spin-echo sequences in axial (slice thickness $4 \mathrm{~mm}$ ) and sagittal (slice thickness $3 \mathrm{~mm}$ ) planes, and, in order to reduce motion artefacts further, a respiratory navigator was positioned on the diaphragm ${ }^{16}$. A diffusion-weighted echo-planar imaging sequence was acquired in the axial plane ( $b$ values $b=0, b=200$, and $b=800 \mathrm{~s} / \mathrm{mm}^{2}$ ). The axial T2W and DW sequences were angled similarly. Detailed MRI sequence parameters are provided in Supplementary File 1.

Image evaluation after nCRT

Two nuclear medicine physicians (hereafter NUC1 and NUC2) retrospectively assessed the FDG$\mathrm{PET} / \mathrm{CT}$ scans, and two radiologists (hereafter RAD1 and RAD2) independently reviewed the DWMRI scans. Observers were blinded for clinical information, pathological outcomes after surgery, and each other's scorings. The two radiologists had only access to the MRI scans (and were blinded to the FDG-PET/CT scans) and vice versa. The likelihood of residual disease in the oesophagus $(y p T+)$ and that of residual nodal disease $(y \mathrm{pN}+)$ was scored using a 5-point confidence level score $(C L S, 1=$ definitely complete response, 2 = probably complete response, $3=$ inconclusive, 4 $=$ probably residual tumour, 5 = definitely residual tumour). This confidence level score was used in multiple studies on clinical response evaluation ${ }^{14,17,18}$. Scoring was done on visual inspection without measurements. Pre-nCRT images were available at the readers' disposal for verification of the tumour location and comparison of amount of uptake or signal intensity. The two scores (for $\mathrm{ypT}+$ and for $\mathrm{ypN+}$ ) were combined into a final residual disease score based on the highest of both scores. For example, if a reader scored CLS 4 on ypT+ and CLS 2 on ypN+, the final residual disease score was scored as CLS 4.

\section{Reference standard}

Histopathologic examination of the resection specimen represented the reference standard and was performed by a dedicated gastrointestinal pathologist. The resection specimen was evaluated in accordance with the 7th edition of the International Union Against Cancer protocol for ypTNM classification ${ }^{19}$. The tumour bed was completely embedded for histopathological analysis. Tumour regression grade (TRG) of the resected primary tumour was assessed according to Mandard, with TRG 1 indicating complete response (ypTO) and TRGs 2-5 indicating residual tumour $(y p T+)^{20}$. ypN stage was dichotomized as ypNO versus ypN+. Overall, a combined pathologic complete response of both the tumour in the oesophagus and lymph nodes was defined as ypTONO, while patients with $\mathrm{ypT}+\mathrm{Nx}$ or ypTON+ were regarded as incomplete responders.

\section{Statistical methods}

Statistical analyses were performed using the Statistical Package for the Social Sciences (SPSS, version 22, Chicago, IL) and STATA (version 11, StataCorp LP, College Station, TX). The diagnostic performance for the detection of residual tumour was calculated by means of the receiver operator 
characteristic (ROC) curve analysis with calculation of the areas under the curve (AUCs). These AUCs were compared between the readers by means of the DeLong test ${ }^{21}$. Diagnostic performance measures (i.e. sensitivity, specificity, positive predictive value (PPV), and negative predictive value (NPV) with 95\% confidence intervals) were computed separately for the detection of ypT+, ypN+, and ypT+ Nx/ypTON+. The agreement of different reader combinations (RAD1 and NUC1, RAD1 and NUC2, RAD2 and NUC1, RAD2 and NUC2) and the diagnostic performance to detect residual tumour after $\mathrm{nCRT}$ were calculated for each of the four reader pairs. Before these analyses were performed, dichotomisation between CLS 2 and CLS 3 was decided as the cut-off between complete response (CLS 1/2) and incomplete response (CLS 3/4/5). Interobserver agreement between the radiologists and interobserver agreement between the nuclear medicine physicians were calculated using Cohen's kappa (0-0.2 poor, 0.21-0.4 fair, 0.41-0.6 moderate, 0.61-0.8 good, and 0.81-1 excellent agreement).

\section{RESULTS}

\section{Patient selection}

Between March 2014 and June 2017, 49 patients were potentially eligible for inclusion. Among them, 16 patients did not meet the inclusion criteria for the following reasons. Two patients switched to definitive CRT during the treatment because of their poor clinical condition; in six patients, the DW-MRI after nCRT was not performed; in four patients, surgical resection was not performed; in three patients, image quality of the DW-MRI after nCRT was insufficient, which impeded the response assessment on DW-MRI; and in one patient, the interval between imaging and surgery exceeded 3 weeks (Fig. 1). This left a final study population of 33 patients meeting all inclusion criteria.

Patient and tumour characteristics

Detailed patient and tumour characteristics are provided in Table 1. The median (interquartile range, IQR) age of the patients was 64 years (53-71), and 29 out of 33 patients (88\%) had adenocarcinomas. Most patients (73\%) had clinical T3 (cT3) stage tumours before the start of $\mathrm{nCRT}^{19}$. After $\mathrm{nCRT}$ and oesophagectomy, the resection specimen showed ypTONO in seven out of 33 patients $(21 \%)$ and residual tumour in 26 patients $(79 \%)$, of whom twelve had ypT+N+, fourteen had ypT+NO, and no patient had ypTON+. This added to a total of 26 ypT+ patients and twelve $y \mathrm{pN}+$ patients. The median (IQR) time interval between completion of $\mathrm{nCRT}$ and surgery was 59 (54-66) days, and between imaging and surgery was 11 (11-13) days. 


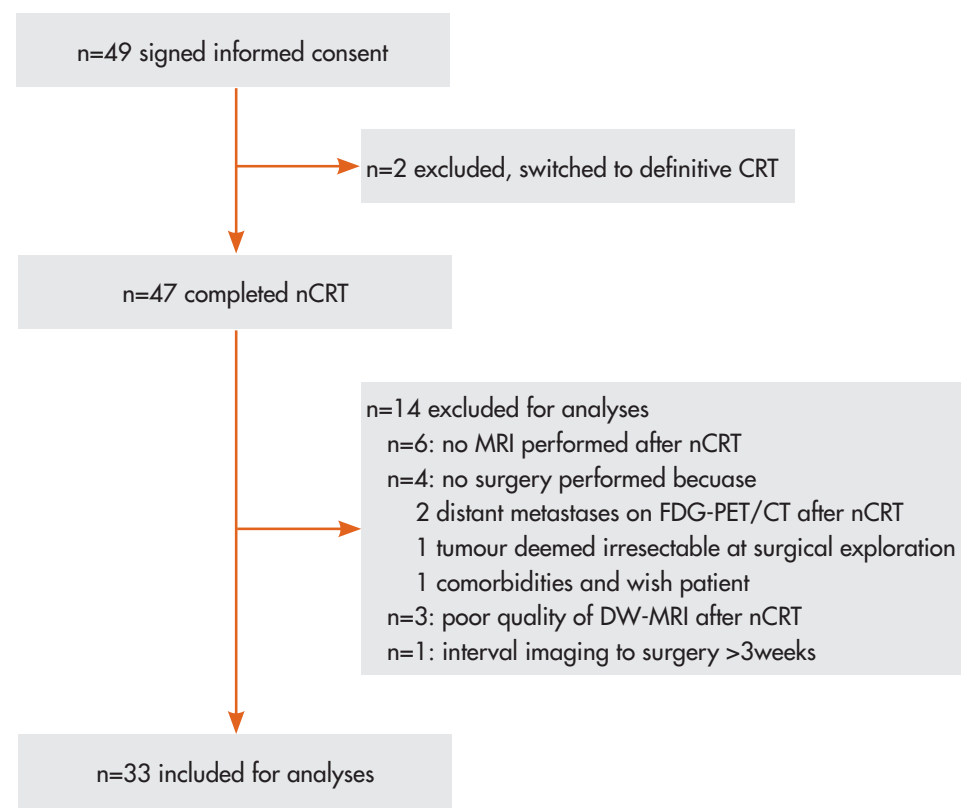

Figure 1. Flow chart

Diagnostic accuracies for the detection of residual tumour in the oesophagus

The AUCs to detect ypT+ were 0.71 and 0.70 on DW-MRI and 0.54 and 0.57 on FDG-PET/ CT. The DW-MRI assessment resulted in sensitivities/specificities of $92 \% / 57 \%$ and $96 \% / 43 \%$ for RAD1 and RAD2, respectively. As for the FDG-PET/CT assessment, sensitivities/specificities were $69 \% / 43 \%$ and $62 \% / 43 \%$ for NUC1 and NUC2, respectively (Table 2).

Diagnostic accuracies for the detection of residual nodal disease

The AUCs to detect ypN+ were 0.81 and 0.89 on DW-MRI and 0.71 and 0.75 on FDG-PET/ CT. The DW-MRI assessment resulted in sensitivities/specificities of $58 \% / 86 \%$ and $67 \% / 86 \%$ for RAD1 and RAD2, respectively. As for the FDG-PET/CT assessment, sensitivities/specificities were $50 \% / 81 \%$ and $42 \% / 86 \%$ for NUC1 and NUC2, respectively (Table 2).

Diagnostic accuracies for the detection of any residual disease

The AUCs to detect any residual disease were 0.74 and 0.70 on DW-MRI for RAD1 and RAD2, respectively. On FDG-PET/CT, these were 0.49 and 0.60 for NUC1 and NUC2, respectively (Table 2). The AUCs of the two MRI readers were significantly higher than the AUC of NUC1 (Table 3). The sensitivities/specificities to detect any residual disease on DW-MRI were $92 \% / 57 \%$ and $96 \% / 43 \%$ for RAD1 and RAD2, respectively. On FDG-PET/CT, the sensitivity to detect any residual disease was $69 \%$ for both readers and specificities were $29 \%$ and $43 \%$ for NUC1 and NUC2, respectively. An example showing discrepant scorings between DW-MRI and FDG-PET/CT is shown in Fig. 2. 
Table 1. Patient and tumour characteristics

\begin{tabular}{|c|c|}
\hline Characteristic & $\mathrm{n}(\%)$ \\
\hline Gender: male & $24(73 \%)$ \\
\hline Median age (years) a & $64(53-71)$ \\
\hline $\begin{array}{l}\text { Tumour location } \\
\text { Middle oesophageal } \\
\text { Lower oesophageal } \\
\text { Gastro-oesophageal junction }\end{array}$ & $\begin{array}{l}3(9 \%) \\
15(45.5 \%) \\
15(45.5 \%)\end{array}$ \\
\hline $\begin{array}{l}\text { Histological tumour type } \\
\text { Squamous cell carcinoma } \\
\text { Adenocarcinoma } \\
\text { Laurén classification for adenocarcinomas: } \\
\text { Intestinal } \\
\text { Diffuse } \\
\text { Mixed } \\
\text { Unknown } \\
\end{array}$ & $\begin{array}{l}4(12 \%) \\
29(88 \%) \\
23 / 29(80 \%) \\
2 / 29(7 \%) \\
3 / 29(10 \%) \\
1 / 29(3 \%) \\
\end{array}$ \\
\hline $\begin{array}{l}\text { Grade of differentiation } \\
\text { Well differentiated } \\
\text { Moderately differentiated } \\
\text { Poorly differentiated } \\
\text { Unknown } \\
\end{array}$ & $\begin{array}{l}2(6 \%) \\
15(45.5 \%) \\
15(45.5 \%) \\
1(3 \%)\end{array}$ \\
\hline $\begin{array}{l}\text { Clinical T-stage }{ }^{b} \\
\text { cT1 } \\
\text { cT2 } \\
\text { cT3 } \\
\end{array}$ & $\begin{array}{l}1(3 \%) \\
8(24 \%) \\
24(73 \%)\end{array}$ \\
\hline $\begin{array}{l}\text { Clinical N-stage }{ }^{b} \\
\text { cN0 } \\
\text { cN1 } \\
\text { cN2 } \\
\text { cN3 } \\
\end{array}$ & $\begin{array}{l}15(45.5 \%) \\
6(18 \%) \\
10(30.5 \%) \\
2(6 \%)\end{array}$ \\
\hline $\begin{array}{l}\text { Pathology after surgery: Mandard b,c } \\
\text { TRG } 1 \text { and NO: ypTONO } \\
\text { TRG } 1 \text { and N1: ypTON+ } \\
\text { TRG } 2 \\
\text { TRG } 3 \\
\text { TRG } 4 \\
\text { TRG } 5\end{array}$ & $\begin{array}{l}7(21 \%) \\
0(0 \%) \\
10(30.5 \%) \\
12(36.5 \%) \\
3(9 \%) \\
1(3 \%)\end{array}$ \\
\hline $\begin{array}{l}\text { Pathology after surgery: } \mathrm{N} \text {-stage }{ }^{b} \\
\text { ypN0 } \\
\text { ypN1 } \\
\text { ypN2 } \\
\text { ypN3 }\end{array}$ & $\begin{array}{l}21(64 \%) \\
4(12 \%) \\
5(15 \%) \\
3(9 \%) \\
\end{array}$ \\
\hline $\begin{array}{l}\text { Time intervals (days) a } \\
\text { End of nCRT until oesophagectomy } \\
\text { End of nCRT until DW-MRI post-nCRT } \\
\text { End of nCRT until FDG-PET/CT post-nCRT } \\
\text { DW-MRI post-nCRT until surgery } \\
\text { FDG-PET/CT post-nCRT until surgery }\end{array}$ & $\begin{array}{l}59(54-66) \\
48(42-51) \\
48(42-51) \\
11(11-13) \\
11(11-13)\end{array}$ \\
\hline \multicolumn{2}{|c|}{$\begin{array}{l}\text { a Data are presented in medians, and data in parentheses represent the interquartile range } \\
\text { b Staging of the oesophageal tumour and lymph nodes according to the 7th edition of the TNM classification }{ }^{19} \\
\text { 'The tumour regression grade of the primary tumour bed was assessed according to Mandard }{ }^{20} \\
\text { n, number of patients; RO, microscopically radical resection; R1, microscopically irradical resection; TRG, tumour regressior } \\
\text { grade; nCRT, neoadjuvant chemoradiotherapy; DW-MRI, diffusion-weighted magnetic resonance imaging; FDG-PET/CT } \\
\text { 18-F fluorodeoxyglucose positron emission tomography }\end{array}$} \\
\hline
\end{tabular}


Table 2. Diagnostic accuracies for the detection of local residual tumour (versus ypTO), residual nodal disease (versus ypNO), and any residual disease (versus ypTONO)

\begin{tabular}{|c|c|c|c|c|c|c|}
\hline Detection of ypT+ & $\begin{array}{l}\text { AUC } 195 \% \\
\text { Cl) }\end{array}$ & Accuracy\%, $n$ & $\begin{array}{l}\text { Sensitivity\% } \\
(95 \% \mathrm{Cl}), \mathrm{n}\end{array}$ & $\begin{array}{l}\text { Specificity\% } \\
(95 \% \mathrm{Cl}), \mathrm{n}\end{array}$ & $\begin{array}{l}\text { NPV\% } \\
(95 \% \mathrm{Cl}), \mathrm{n}\end{array}$ & $\begin{array}{l}\text { PPV\% } \\
(95 \% \mathrm{Cl}), \mathrm{n}\end{array}$ \\
\hline RADI (MRI) & $\begin{array}{l}0.71 \\
(0.47-0.96)\end{array}$ & $85,28 / 33$ & $\begin{array}{l}92(73-99), \\
24 / 26\end{array}$ & $\begin{array}{l}57(20-88), \\
4 / 7\end{array}$ & $\begin{array}{l}67(24-94), \\
4 / 6\end{array}$ & $\begin{array}{l}88 \text { (70-97), } \\
24 / 27\end{array}$ \\
\hline RAD2 (MRI) & $\begin{array}{l}0.70 \\
(0.46-0.96)\end{array}$ & $85,28 / 33$ & $\begin{array}{l}96(78-100), \\
25 / 26\end{array}$ & $\begin{array}{l}43(12-80), \\
3 / 7\end{array}$ & $\begin{array}{l}75(22-99), \\
3 / 4\end{array}$ & $\begin{array}{l}86 \text { (67-95), } \\
25 / 29\end{array}$ \\
\hline $\mathrm{NUCl}$ (PET) & $\begin{array}{l}0.54 \\
(0.29-0.79)\end{array}$ & $64,21 / 33$ & $\begin{array}{l}69(48-84), \\
18 / 26\end{array}$ & $\begin{array}{l}43(12-80), \\
3 / 7\end{array}$ & $\begin{array}{l}27(7-61), \\
3 / 11\end{array}$ & $\begin{array}{l}82(59-94), \\
18 / 22\end{array}$ \\
\hline NUC2 (PET) & $\begin{array}{l}0.57 \\
(0.31-0.84)\end{array}$ & $58,19 / 33$ & $\begin{array}{l}62(41-79), \\
16 / 26\end{array}$ & $\begin{array}{l}43(12-80), \\
3 / 7\end{array}$ & $\begin{array}{l}23(6-54), \\
3 / 13\end{array}$ & $\begin{array}{l}80(56-93), \\
16 / 20\end{array}$ \\
\hline \multicolumn{7}{|l|}{ Detection of ypN+ } \\
\hline RADI (MRI) & $\begin{array}{l}0.89 \\
(0.77-1.00)\end{array}$ & $76,25 / 33$ & $\begin{array}{l}58(29-83), \\
7 / 12\end{array}$ & $\begin{array}{l}86(63-96), \\
18 / 21\end{array}$ & $\begin{array}{l}78(56-92) \\
18 / 23\end{array}$ & $\begin{array}{l}70(35-92), \\
7 / 10\end{array}$ \\
\hline RAD2 (MRI) & $\begin{array}{l}0.81 \\
(0.66-0.97)\end{array}$ & $79,26 / 33$ & $\begin{array}{l}67(35-89), \\
8 / 12\end{array}$ & $\begin{array}{l}86(63-96), \\
18 / 21\end{array}$ & $\begin{array}{l}82(59-94) \\
18 / 22\end{array}$ & $\begin{array}{l}73 \text { (39-93), } \\
8 / 11\end{array}$ \\
\hline $\mathrm{NUCl}$ (PET) & $\begin{array}{l}0.75 \\
(0.56-0.93)\end{array}$ & $70,23 / 33$ & $\begin{array}{l}50(22-78) \\
6 / 12\end{array}$ & $\begin{array}{l}81(57-94), \\
17 / 21\end{array}$ & $\begin{array}{l}74(51-89) \\
17 / 23\end{array}$ & $\begin{array}{l}60(27-86), \\
6 / 10\end{array}$ \\
\hline NUC2 (PET) & $\begin{array}{l}0.71 \\
(0.52-0.91) \\
\end{array}$ & $70,23 / 33$ & $\begin{array}{l}42(16-71), \\
5 / 12\end{array}$ & $\begin{array}{l}86(63-96), \\
18 / 21\end{array}$ & $\begin{array}{l}72(50-87) \\
18 / 25\end{array}$ & $\begin{array}{l}63(26-90), \\
5 / 8\end{array}$ \\
\hline \multicolumn{7}{|c|}{ Detection of ypT+Nx/ypTON+ } \\
\hline RADI (MRI) & $\begin{array}{l}0.74 \\
(0.50-0.97)\end{array}$ & $85,28 / 33$ & $\begin{array}{l}92(74-99), \\
24 / 26\end{array}$ & $\begin{array}{l}57(20-88), \\
4 / 7\end{array}$ & $\begin{array}{l}67(24-94), \\
4 / 6\end{array}$ & $\begin{array}{l}89 \text { (70-97), } \\
24 / 27\end{array}$ \\
\hline RAD2 (MRI) & $\begin{array}{l}0.70 \\
(0.46-0.94)\end{array}$ & $85,28 / 33$ & $\begin{array}{l}96(78-100), \\
25 / 26\end{array}$ & $\begin{array}{l}43(12-80), \\
3 / 7\end{array}$ & $\begin{array}{l}75 \text { (22-99), } \\
3 / 4\end{array}$ & $\begin{array}{l}86 \text { (67-95), } \\
25 / 29\end{array}$ \\
\hline $\mathrm{NUCl}$ (PET) & $\begin{array}{l}0.49 \\
(0.25-0.73)\end{array}$ & $61,20 / 33$ & $\begin{array}{l}69(48-85), \\
18 / 26\end{array}$ & $\begin{array}{l}29(5-70), \\
2 / 7\end{array}$ & $\begin{array}{l}20(4-56), \\
2 / 10\end{array}$ & $\begin{array}{l}78(56-92), \\
18 / 23\end{array}$ \\
\hline NUC2 (PET) & $\begin{array}{l}0.60 \\
(0.34-0.86)\end{array}$ & $64,21 / 33$ & $\begin{array}{l}69(48-85) \\
18 / 26\end{array}$ & $\begin{array}{l}43(12-80), \\
3 / 7\end{array}$ & $\begin{array}{l}27(7-61), \\
3 / 11\end{array}$ & $\begin{array}{l}82(59-94), \\
18 / 22\end{array}$ \\
\hline
\end{tabular}

AUC, area under the receiver operating characteristic curve; $M R I$, magnetic resonance imaging; PET, positron emission tomography; NPV, negative predictive value; PPV, positive predictive value; $\mathrm{Cl}$, confidence interval; RADI, radiologist 1; RAD2, radiologist 2; NUC1, nuclear medicine physician 1; NUC2, nuclear medicine physician 2; n, number of patients

Table 3. Comparison of the areas under the curves for the detection of any residual disease $(y p T+N x / y p T O N+)$ between the radiologists on DW-MRI and the nuclear medicine physicians on FDG-PET/CT

\begin{tabular}{|c|c|c|c|c|}
\hline & RADI , AUC 0.74 & RAD2, AUC 0.70 & $\mathrm{NUC1}$, AUC 0.49 & NUC2, AUC 0.60 \\
\hline RADI, AUC 0.74 & $x$ & 0.7516 & $0.0020^{*}$ & 0.1645 \\
\hline RAD2, AUC 0.70 & $x$ & $x$ & $0.0449^{*}$ & 0.3550 \\
\hline $\mathrm{NUCl}$, AUC 0.49 & $x$ & $x$ & $x$ & 0.2045 \\
\hline NUC2, AUC 0.60 & $x$ & $x$ & $x$ & $x$ \\
\hline \multicolumn{5}{|c|}{$\begin{array}{l}\text { This table shows the comparison of the areas under the curve between the readers, measured by using the Delong Test }{ }^{21} \text {. } \\
\text { Each value in the table represents the calculated probability (p value). Significant differences are marked with an asterisk } \\
\left({ }^{*}\right) \text {. DW-MRI, diffusion-weighted magnetic resonance imaging; FDG-PET/CT, positron emission tomography; AUC, area } \\
\text { under the receiver operating characteristic curve; RAD1, radiologist 1; RAD2, radiologist 2; NUC1, nuclear medicine } \\
\text { physician 1; NUC2, nuclear medicine physician } 2\end{array}$} \\
\hline
\end{tabular}


Combined MRI and FDG-PET/CT assessment for the detection of any residual disease

The diagnostic performance of 4 different pairs of radiologists and nuclear medicine physicians to detect residual disease after nCRT based on the separate, blinded scorings is shown in Table 4. In 64 to $79 \%$ of patients, the radiologist and nuclear medicine physician agreed on either the presence of any residual disease (ypT+Nx/ypTON+) or complete response (ypTONO). The sensitivities ranged from 94 to $100 \%$ with specificities ranging from 33 to $50 \%$. If readers on both modalities agreed on the presence of residual disease (CLS 3/4/5), residual tumour was found in 82 to $85 \%$ of the surgical resection specimens (PPV, Table 4).

Interobserver agreement

The agreement between the radiologists on DW-MRI was moderate $(\kappa=0.53)$, and the agreement between the nuclear medicine physicians on FDG-PET/CT was fair ( $\kappa=0.37$ ).

Table 4. Diagnostic accuracies in cases the radiologist and nuclear medicine physician agreed on the presence of any residual disease (both CLS 3/4/5) or complete response (both CLS 1/2)

\begin{tabular}{lllllll}
\hline $\begin{array}{l}\text { Detection of } \\
\text { ypT+Nx/ypTON+ }\end{array}$ & \multirow{2}{*}{$\begin{array}{l}\text { Agreement } \\
\text { Accuracy\%, n }\end{array}$} & $\begin{array}{l}\text { Sensitivity\% } \\
(95 \% \mathrm{Cl}), \mathrm{n}\end{array}$ & $\begin{array}{l}\text { Specificity\% } \\
(95 \% \mathrm{Cl}), \mathrm{n}\end{array}$ & $\begin{array}{l}\mathrm{NPV} \% \\
(95 \% \mathrm{Cl}), \mathrm{n}\end{array}$ & $\begin{array}{l}\text { PPV\% } \\
(95 \% \mathrm{Cl}), \mathrm{n}\end{array}$ \\
\hline RAD1 \& NUC1 & $21 / 33(64 \%)$ & $86,18 / 21$ & $\begin{array}{l}100(52-91), \\
16 / 16\end{array}$ & $\begin{array}{l}40(7-83), \\
2 / 5\end{array}$ & $\begin{array}{l}100(20- \\
100), 2 / 2\end{array}$ & $\begin{array}{l}84(60-96), \\
16 / 19\end{array}$ \\
\hline RAD1 \& NUC2 & $24 / 33(73 \%)$ & $83,20 / 24$ & $\begin{array}{l}94(71-100), \\
17 / 18\end{array}$ & $50(14-86)$, & $75(22-99)$, & $85(61-96)$, \\
& & & $95 / 4$ & $3 / 4$ & $17 / 20$ \\
\hline RAD2 \& NUC1 & $25 / 33(76 \%)$ & $80,20 / 25$ & $95(72-100)$, & $33(6-76)$, & $67(13-98)$, & $82(59-94)$, \\
& & & $18 / 19$ & $2 / 6$ & $2 / 3$ & $18 / 22$ \\
\hline RAD2 \& NUC2 & $26 / 33(79 \%)$ & $81,21 / 26$ & $95(72-100)$, & $43(12-80)$, & $75(22-99)$, & $82(59-94)$, \\
& & & $18 / 19$ & $3 / 7$ & $3 / 4$ & $18 / 22$ \\
\hline
\end{tabular}

MRI, magnetic resonance imaging; PET, positron emission tomography; Sens, sensitivity; Spec, specificity; NPV, negative predictive value; PPV, positive predictive value; $\mathrm{Cl}$, confidence interval; RAD1, radiologist 1; RAD2, radiologist 2; $\mathrm{NUCl}$, nuclear medicine physician 1; NUC2, nuclear medicine physician 2; n, number of patients 

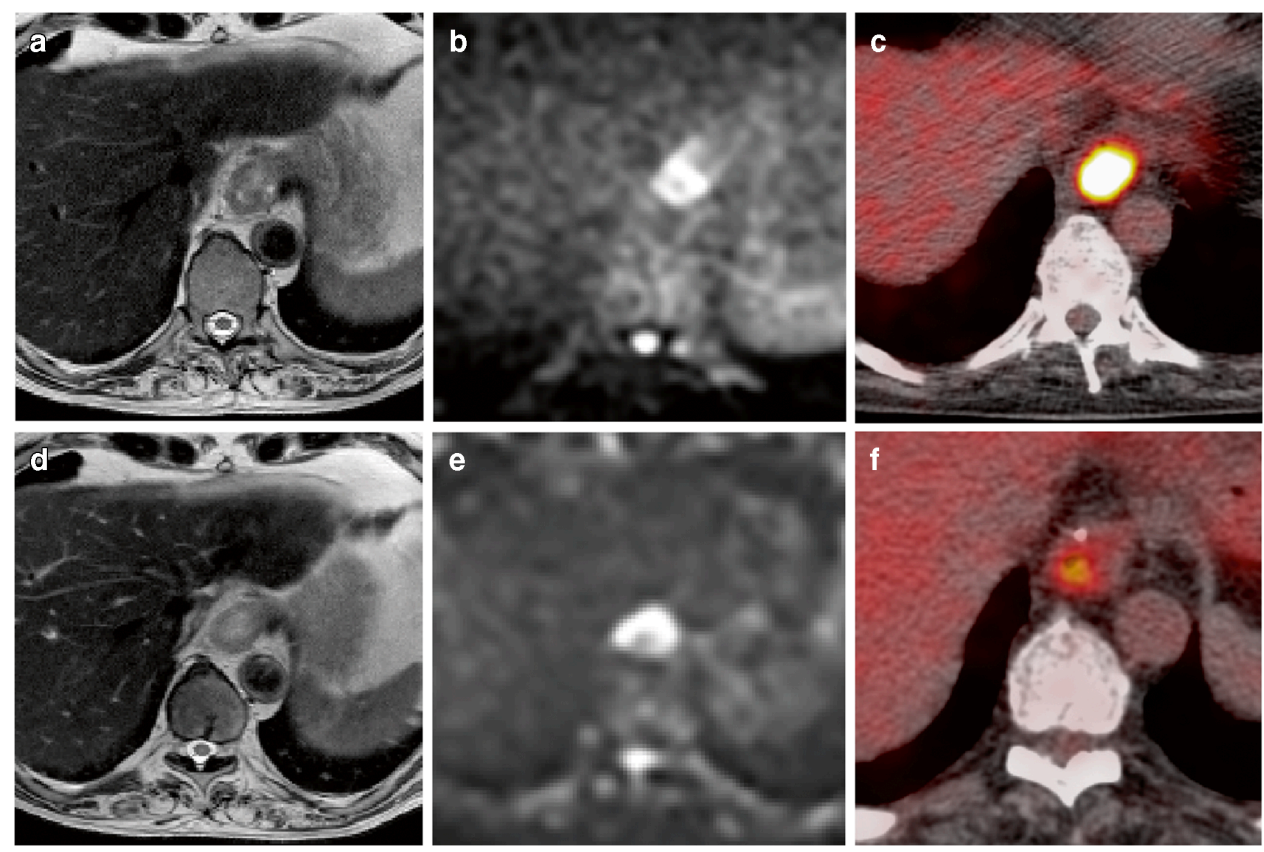

Figure 2. Patient example

A 72-year-old male patient with a cT3N2MO oesophageal adenocarcinoma treated with neoadjuvant chemoradiotherapy (nCRT) and oesophagectomy. Histopathology revealed residual tumour (tumour regression grade 3, ypT2N2). The T2-weighted axial image before (a) and 7 weeks after (d) nCRT shows an isointense oesophageal wall. The corresponding $b=800$ diffusionweighted images before (b) and after (e) nCRT demonstrate a hyperintense signal, highly suspicious for residual tumour (assessed as CLS 4 and CLS 5 by the MRI readers). The tumour showed high uptake of fluorodeoxyglucose (FDG) before (c) the start of $n C R T$, whereas there was only minimal FDG uptake which was spread throughout the oesophageal wall after completion of $n C R T(f)$ assessed as CLS 2 and CLS 3 by the FDG-PET/CT readers.

\section{DISCUSSION}

This study is the first prospective study comparing the performance of DW-MRI with that of FDG$\mathrm{PET} / \mathrm{CT}$ in detecting residual disease after $\mathrm{nCRT}$ for oesophageal cancer. The results showed that DW-MRI outperformed FDG-PET/CT with regard to the local assessment of response to nCRT. The sensitivity to detect any locoregional residual tumour on DW-MRI was $92-96 \%$ versus $69 \%$ for both readers on FDG-PET/CT. Interestingly, when the radiologist and nuclear medicine physician agreed on the presence of a residual tumour, the sensitivity and specificity were comparable to a single assessment by the radiologists on DW-MRI. This suggests that FDG-PET/CT does not add to DW-MRI for the response assessment of the primary tumour. The high sensitivities and PPVs suggest 
that DW-MRI could be of value in detecting residual tumour after $\mathrm{nCRT}$ if organ preservation in oesophageal cancer patients is being considered. However, the low specificities of DW-MRI imply a chance of missing complete responders for whom an organ-preserving treatment would have been an option.

Previous studies on DW-MRI for the response assessment after nCRT in oesophageal cancer have focussed mainly on the apparent diffusion coefficient (ADC) derived from DW-MRI, which represents a quantitative measure of the diffusion of water ${ }^{22-35}$. Theoretically, tumorous tissue with restricted diffusion (and a low ADC value) can be differentiated from fibrosis without diffusion restriction. In a systematic review, the relative increase of $A D C$ during treatment was significantly higher in good versus poor responders in most (five out of eight) included studies ${ }^{36}$. However, response assessment after treatment using the ADC is not robust, probably due to the various methods used to draw the region of interest, the practice variations regarding the scanners and scan protocols, post-processing variations, and the choice of cut-off values. Conversely, a subjective response assessment may be more robust for the response assessment of the primary tumour after $\mathrm{nCRT}$, as was shown in a previously published study in oesophageal cancer patients and in rectal cancer ${ }^{14}$. In the latter, DW-MRI is used in addition to endoscopy in the selection process for the organsaving treatment for patients with a clinical complete response to chemoradiotherapy $12,13,17,37$. Interestingly, a previous study on oesophageal cancer showed that DW-MRI also had additional value to endoscopy and endoscopic ultrasound (EUS) for the detection of residual tumour after $\mathrm{nCRT}^{38}$.

The main drawback of FDG-PET/CT in the local response assessment after nCRT is the low specificity, which may be explained by the presence of (radiation-induced) oesophagitis, masking a complete tumour response $\mathrm{e}^{39,40}$. In our study, the median interval between completion of nCRT and FDG-PET/CT acquisition was 48 days, and prolonging this interval might overcome this problem. Although it is known that the presence of oesophagitis is correlated to the radiation dose and usually becomes apparent after about 2 weeks of radiotherapy, it is yet unclear how long oesophagitis persists on FDG-PET/CT after $n C R T^{41-43}$. Also, the detection of small residual tumour lesions appears challenging on FDG-PET/CT ${ }^{44}$. Theoretically, if residual tumour cells after $n C R T$ in the primary tumour bed are clustered together, FDG-PET/CT may be able to detect these clusters, whereas a scattered localisation throughout the oesophageal wall may complicate the detection due to the low spatial resolution of FDG-PET/CT, resulting in moderate sensitivities.

Another focus in our study was the detection of residual nodal disease on imaging after $\mathrm{nCRT}$. The high specificities (81-86\%) and NPVs (72-82\%) suggest that the absence of suspect lymph nodes on imaging after $\mathrm{nCRT}$ may yield predictive value for a true (pathological) absence of nodal disease (ypNO). The AUCs were remarkably high (0.81 and 0.89) on DW-MRI, even though the hypothesis was that the differentiation between malignant and benign lymph nodes on DW-MRI would be challenging as both show diffusion restriction. On FDG-PET/CT, the small size of remaining mediastinal tumour-positive lymph nodes may have complicated response 
assessment. Despite these challenges for FDG-PET/CT, the readers reached AUCs of 0.75 and 0.71 for residual nodal disease detection. These results concur with a recent study in patients with oesophageal squamous cell cancer, in which a residual nodal disease on FDG-PET/CT after nCRT was significantly associated with the presence of tumour-positive lymph nodes on pathological examination ${ }^{45}$.

Currently, clinical response assessment after nCRT using a combination of endoscopy, EUS, and FDG-PET/CT is being explored in prospective trials ${ }^{5,46}$. The piffall of endoscopic biopsies is the high risk of false-negative findings, due to submucosal residual tumour and sampling error. Furthermore, on EUS, the differentiation between benign and malignant wall thickening after chemoradiotherapy has been reported to be difficult ${ }^{47}$. Therefore, an imaging modality with a high sensitivity and NPV is needed to improve response assessment by endoscopic evaluation. The current study showed that these values were both higher on DW-MRI as compared with FDG-PET/CT. No additional benefit for local response assessment was even found by adding FDG-PET/CT to DW-MRI if performed 6-8 weeks after completion of CRT. However, for the detection of distant metastases, FDG-PET/ CT still seems essential. In the future, wholebody DW-MRI may be used for this purpose, but this was beyond the scope of this study ${ }^{48}$. A multimodal approach using a combination of FDG-PET/ CT, DW-MRI, and endoscopy with EUS will likely be the ideal method to identify clinical complete responders to neoadjuvant chemoradiotherapy.

Some limitations of the current study must be mentioned. Although this was the first study comparing clinical response assessment on FDG-PET/CT and DW-MRI after $\mathrm{nCRT}$ in oesophageal cancer patients, the sample size was relatively small. Also, only the locoregional lymph nodes within the field of view of the DW-MRI were assessed because of scan time restrictions. Furthermore, the assessment of FDG-PET/CT might be influenced by various system settings at acquisition before nCRT. However, the post-nCRT FDG-PET/CT scans were performed on the same scanner in all patients. Whereas for quantitative imaging analyses the exact acquisition parameter settings could be an issue, the variation in pretreatment FDG-PET/CT acquisition can be accepted for subjective response assessment studies.

In conclusion, this study showed that DW-MRI reached higher diagnostic accuracies than FDG$\mathrm{PET} / \mathrm{CT}$ for the detection of local residual tumour in oesophageal cancer patients at 6 to 8 weeks after nCRT. 


\section{REFERENCES}

1. Bray F, Ferlay J, Soeriomataram I, Siegel RL, Torre LA, Jemal A. Global cancer statistics 2018: GLOBOCAN estimates of incidence and mortality worldwide for 36 cancers in 185 countries. CA: a cancer journal for clinicians 2018;68:394-424.

2. Sjoquist $K M$, Burmeister $B H$, Smithers $B M$, et al. Survival after neoadjuvant chemotherapy or chemoradiotherapy for resectable oesophageal carcinoma: an updated meta-analysis. Lancet Oncol $2011 ; 12: 681-92$.

3. Biermann K, Van Lanschot JJB, Rozema T, et al. Preoperative Chemoradiotherapy for Esophageal or Junctional Cancer. N Engl J Med 2012;366:2074-84.

4. clinicaltrials.gov [internet]. Centre Hospitalier Universitaire Dijon: Comparison of Systematic Surgery Versus Surveillance and Rescue Surgery in Operable Oesophageal Cancer With a Complete Clinical Response to Radiochemotherapy (Esostrate) NCTO2551458 2015 [updated 1/12/16]. Available from: https://clinicaltrials.gov/show/ NCT02551458.

5. Noordman BJ, Wijnhoven BPL, Lagarde $S M$, et al. Neoadjuvant chemoradiotherapy plus surgery versus active surveillance for oesophageal cancer: a stepped-wedge cluster randomised trial. BMC Cancer 2018;18:142.

6. Kroese $T E$, Goense L, van Hillegersberg R, et al. Detection of distant interval metastases after neoadjuvant therapy for esophageal cancer with 18F-FDG PET(/CT): a systematic review and meta-analysis. Dis Esophagus 2018;31.

7. Stiekema J, Vermeulen D, Vegt $E$, et al. Detecting interval metastases and response assessment using 18F-FDG PET/CT after neoadjuvant chemoradiotherapy for esophageal cancer. Clin Nucl Med 2014;39:862-7.

8. de Gouw D, Klarenbeek BR, Driessen M, et al. Detecting Pathological Complete Response in Esophageal Cancer after Neoadjuvant Therapy Based on Imaging Techniques: A Diagnostic Systematic Review and Meta-Analysis. J Thorac Oncol 2019;14:1156-71.

9. Eyck BM, Onstenk BD, Noordman BJ, et al. Accuracy of Detecting Residual Disease After Neoadjuvant Chemoradiotherapy for
Esophageal Cancer. Ann Surg 2020;271:24556.

10. Koh DM, Collins DJ. Diffusion-weighted MRI in the body: applications and challenges in oncology. AJR Am J Roentgen 2007; 188:162235 .

11. Le Bihan D, Poupon C, Amadon A, Lethimonnier F. Artifacts and pitfalls in diffusion MRI. J Magn Reson Imaging 2006;24:478-88.

12. Lambregts DM, Rao SX, Sassen S, et al. MRI and Diffusion-weighted MRI Volumetry for Identification of Complete Tumor Responders After Preoperative Chemoradiotherapy in Patients With Rectal Cancer: A Bi-institutional Validation Study. Ann Surg 2015;262:1034-9.

13. Maas $M$, Lambregts DM, Nelemans PJ, et al. Assessment of Clinical Complete Response After Chemoradiation for Rectal Cancer with Digital Rectal Examination, Endoscopy, and MRI: Selection for Organ-Saving Treatment. Ann Surg Oncol 2015;22:3873-80.

14. Vollenbrock SE, Voncken FEM, van Dieren $J M$, et al. Diagnostic performance of MRI for assessment of response to neoadjuvant chemoradiotherapy in oesophageal cancer. $\mathrm{Br}$ J Surg 2019;106:596-605.

15. Boellaard R, Delgado-Bolton R, Oyen WJ, et al. FDG PET/CT: EANM procedure guidelines for tumour imaging: version 2.0. Eur J Nucl Med Mol Imaging 2015;42:328-54.

16. Meijer GJ, Moerland MA, van Lier ALHMW, et al. Quantification of Esophageal Tumor Motion on Cine-Magnetic Resonance Imaging. Int J Radiat Oncol Biol Phys 2014;88:419-24.

17. Lambregts DM, Vandecaveye V, Barbaro B, et al. Diffusion-weighted MRI for selection of complete responders after chemoradiation for locally advanced rectal cancer: a multicenter study. Ann Surg Oncol 2011;18:2224-31.

18. Noordman BJ, Spaander MCW, Valkema $R$, et al. Detection of residual disease after neoadjuvant chemoradiotherapy for oesophageal cancer (preSANO): a prospective multicentre, diagnostic cohort study. Lancet Oncol 2018;19:965-74.

19. Rice TW, Blackstone EH, Rusch VW. 7th edition 
of the AJCC Cancer Staging Manual: esophagus and esophagogastric junction. Ann Surg Oncol 2010;17:1721-4.

20. Mandard AM, Dalibard F, Mandard JC, et al Pathologic assessment of tumor regression after preoperative chemoradiotherapy of esophageal carcinoma. Clinicopathologic correlations. Cancer 1994;73:2680-6.

21. Delong ER, Delong DM, Clarke-Pearson DL. Comparing the areas under two or more correlated receiver operating characteristic curves: a nonparametric approach. Biometrics 1988;44:837-45

22. Aoyagi T, Shuto K, Okazumi S, Shimada H, Kazama T, Matsubara H. Apparent diffusion coefficient values measured by diffusion-weighted imaging predict chemoradiotherapeutic effect for advanced esophageal cancer. Dig Surg 2011 ;28:252-7.

23. De Cobelli F, Giganti F, Orsenigo E, et al. Apparent diffusion coefficient modifications in assessing gastro-oesophageal cancer response to neoadjuvant treatment: comparison with tumour regression grade at histology. Eur Radiol 2013;23:2165-74.

24. Imanishi S, Shuto K, Aoyagi T, Kono T, Saito $\mathrm{H}$, Matsubara H. Diffusion-weighted magnetic resonance imaging for predicting and detecting the early response to chemoradiotherapy of advanced esophageal squamous cell carcinoma. Dig Surg 2013;30:240-8.

25. Weber MA, Bender K, von Gall CC, et al. Assessment of diffusion-weighted MRI and 18F-fluoro-deoxyglucose PET/CT in monitoring early response to neoadjuvant chemotherapy in adenocarcinoma of the esophagogastric junction. J Gastrointestin Liver Dis 2013;22:4552.

26. Kwee RM, Dik AK, Sosef MN, et al. Interobserver reproducibility of diffusion-weighted MRI in monitoring tumor response to neoadjuvant therapy in esophageal cancer. PLoS One 2014;9:e92211.

27. Liu S, Zhen F, Sun N, et al. Apparent diffusion coefficient values detected by diffusion-weighted imaging in the prognosis of patients with locally advanced esophageal squamous cell carcinoma receiving chemoradiation. Onco Targets Ther 2016;9:5791-6.

28. Wang L, Liu L, Han C, etal. The diffusion-weighted magnetic resonance imaging (DWI) predicts the early response of esophageal squamous cell carcinoma to concurrent chemoradiotherapy. Radiother Oncol 2016;121:246-51.

29. Fang $P$, Musall BC, Son JB et al. Multimodal Imaging of Pathologic Response to Chemoradiation in Esophageal Cancer. Int J Radiat Oncol Biol Phys 2018;102:996-1001.

30. Guo L, Zhang L, Zhao J. CT scan and magnetic resonance diffusion-weighted imaging in the diagnosis and treatment of esophageal cancer. Oncol Lett 2018;16:7117-22.

31. Heethuis SE, Goense L, van Rossum PSN, et al. DW-MRI and DCE-MRI are of complementary value in predicting pathologic response to neoadjuvant chemoradiotherapy for esophageal cancer. Acta Oncol 2018;57:1201-8.

32. Kozumi $M$, Ota $H$, Yamamoto $T$, et al. Oesophageal squamous cell carcinoma: histogram-derived ADC parameters are not predictive of tumour response to chemoradiotherapy. Eur Radiol 2018;28:4296305.

33. Li FP, Wang $\mathrm{H}$, Hou J, et al. Utility of intravoxel incoherent motion diffusion-weighted imaging in predicting early response to concurrent chemoradiotherapy in oesophageal squamous cell carcinoma. Clin Radiol 2018;73:756 e17e26.

34. Li QW, Qiu B, Wang B, et al. Prediction of pathologic responders to neoadjuvant chemoradiotherapy by diffusion-weighted magnetic resonance imaging in locally advanced esophageal squamous cell carcinoma: a prospective study. Dis Esophagus 2018;31:1090.

35. Zheng $\mathrm{H}$, Ren $\mathrm{W}$, Pan $\mathrm{X}$, et al. Role of intravoxel incoherent motion MRI in early assessment of the response of esophageal squamous cell carcinoma to chemoradiotherapy: A pilot study. J Magn Reson Imaging 2018;48:349-58.

36. Vollenbrock SE, Voncken FEM, Bartels LW, Beets-Tan RGH, Bartels-Rutten A. Diffusionweighted MRI with ADC mapping for response prediction and assessment of oesophageal cancer: A systematic review. Radiother Oncol 2020;142:17-26.

37. van der Paardt MP, Zagers MB, Beets-Tan RG, Stoker J, Bipat S. Patients who undergo preoperative chemoradiotherapy for locally 
advanced rectal cancer restaged by using diagnostic MR imaging: a systematic review and meta-analysis. Radiology 2013;269:101-12.

38. Vollenbrock SE, van Dieren JM, Voncken FEM, et al. Added value of MRI to endoscopic and endosonographic response assessment after neoadjuvant chemoradiotherapy in oesophageal cancer. Eur Radiol 2020.

39. Bhargava P, Reich P, Alavi A, Zhuang H. Radiation-induced esophagitis on FDG PET imaging. Clin Nucl Med 2003;28:849-50.

40. Mehmood Q, Sun A, Becker N, et al. Predicting Radiation Esophagitis Using 18F-FDG PET During Chemoradiotherapy for Locally Advanced Non-Small Cell Lung Cancer. J Thorac Oncol 2016;11:213-21.

41. Niikamp J, Rossi M, Lebesque J, et al. Relating acute esophagitis to radiotherapy dose using FDG-PET in concurrent chemo-radiotherapy for locally advanced non-small cell lung cancer. Radiother Oncol 2013;106:118-23.

42. Bruzzi JF, Munden RF, Truong MT, et al. PET/ CT of esophageal cancer: its role in clinical management. Radiographics 2007;27:163552.

43. Hautzel H, Muller-Gartner HW. Early changes in fluorine-18-FDG uptake during radiotherapy. Journal of nuclear medicine : official publication, Society of Nuclear Medicine 1997;38:1384-6.

44. Soret M, Bacharach SL, Buvat I. Partial-volume effect in PET tumor imaging. J Nucl Med 2007;48:932-45

45. Hamai $Y$, Hihara J, Emi $M$, et al. Preoperative prediction of a pathologic complete response of esophageal squamous cell carcinoma to neoadjuvant chemoradiotherapy. Surgery 2018.

46. Eloubeidi MA, Cerfolio RJ, Bryant AS, Varadarajulu S. Efficacy of endoscopic ultrasound in patients with esophageal cancer predicted to have NO disease. Eur J Cardiothorac Surg 2011 ; 40:636-41.

47. Griffin JM, Reed CE, Denlinger CE. Utility of restaging endoscopic ultrasound after neoadjuvant therapy for esophageal cancer. Ann Thorac Surg 2012;93:1855-9; discussion 60.

48. Malik V, Harmon M, Johnston C, et al. Whole
Body MRI in the Staging of Esophageal Cancer-A Prospective Comparison with Whole Body 18F-FDG PET-CT. Dig Surg 2015;32:397-408. 


\section{SUPPLEMENTARY FILES}

Suppl. Table 1. MRI parameters

\begin{tabular}{|c|c|c|c|}
\hline \multirow[b]{2}{*}{ Imaging plane } & \multicolumn{2}{|c|}{ T2W-MRI } & \multirow{2}{*}{$\frac{\text { DW-MRI }}{\text { Axial }}$} \\
\hline & Axial & Sagittal & \\
\hline Slice thickness $(\mathrm{mm})$ & 4 & 3 & 4 \\
\hline Field of view $\left(\mathrm{mm}^{3}, \mathrm{APxRLxFH}\right)$ & $225 \times 225 \times 180$ & $150 \times 60 \times 225$ & $260 \times 520 \times 200$ \\
\hline In-plane resolution $\left(\mathrm{mm}^{2}\right)$ & $0.67 \times 0.67$ & $0.70 \times 0.70$ & $3.25 \times 3.25$ \\
\hline Repetition time (ms) & $1454-1938$ & $1431-1463$ & $7421-7503$ \\
\hline Echo time (ms) & 100 & 100 & 77 \\
\hline Echo train length & 39 & 22 & 71 \\
\hline b-values $\left(\mathrm{s} / \mathrm{mm}^{2}\right)$ & - & - & $0,200,800$ \\
\hline NSA & 2 & 2 & $4,4,12$ \\
\hline $\begin{array}{l}\text { Respiratory triggering } \\
\text { (navigator) }\end{array}$ & Yes & Yes & No \\
\hline Fat suppression technique & - & - & SPIR \\
\hline
\end{tabular}




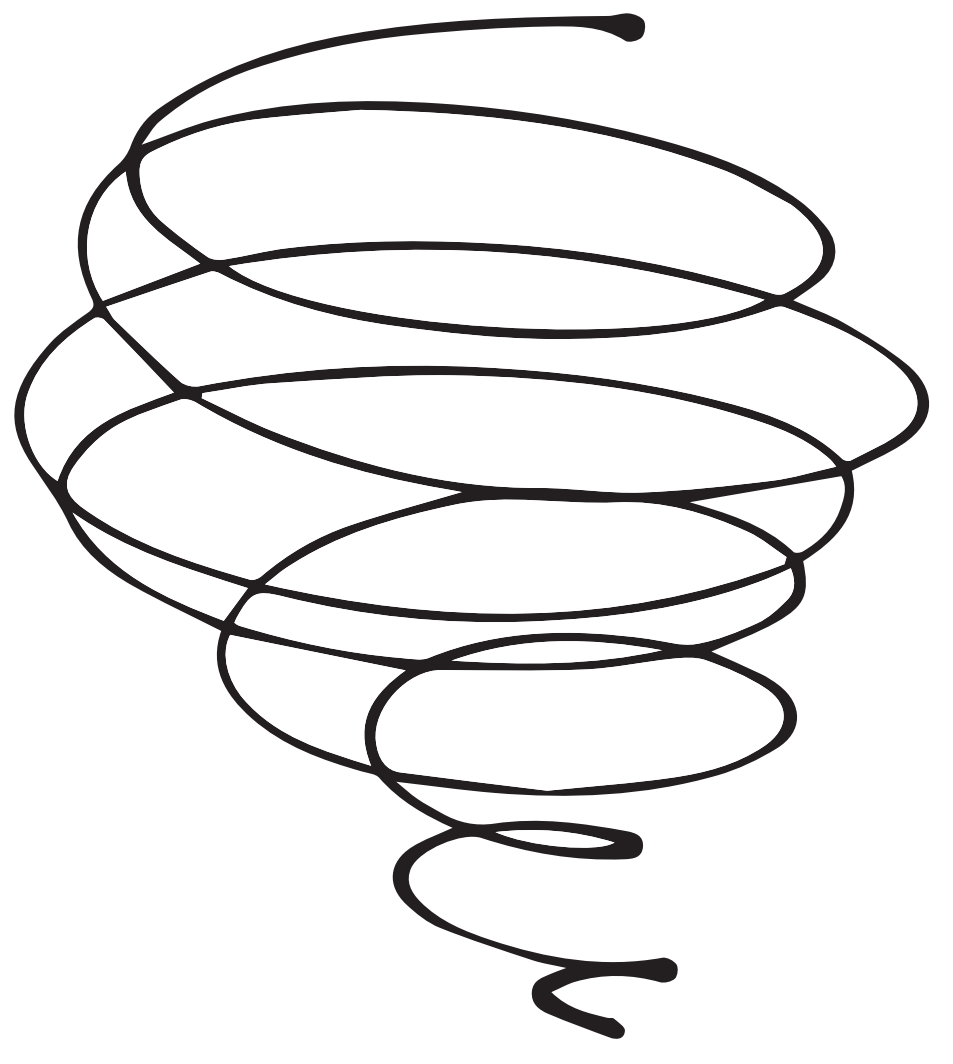




\title{
CHAPTER 5
}

\section{Added value of MRI to endoscopic and endosonographic response assessment after neoadjuvant chemoradiotherapy in oesophageal cancer}

European Radiology; May 2020 (Volume 30, Issue 5, p2425-2434)

\author{
Sophie E. Vollenbrock \\ Jolanda M. van Dieren \\ Francine E.M. Voncken \\ Sietze T. van Turenhout \\ Liudmila L. Kodach \\ Koen J. Hartemink \\ Johanna W. van Sandick \\ Berthe M.P. Aleman \\ Regina G.H. Beets-Tan \\ Annemarieke Bartels-Rutten
}




\begin{abstract}
Objectives

In order to select oesophageal cancer patients after neoadjuvant chemoradiotherapy (nCRT) for organ-preserving treatment instead of surgery, a high diagnostic accuracy is required. The aim of this study was to evaluate whether MRI had additional value to gastroscopy with biopsies and endosonographic ultrasound (EUS) with fine needle aspiration (FNA) for the detection of residual tumour after nCRT.
\end{abstract}

Methods

Twenty-two patients with oesophageal cancer eligible for nCRT followed by oesophagectomy were prospectively included. All patients underwent (T2- and diffusion-weighted) MRI and gastroscopy+EUS before and after nCRT. Histopathology after oesophagectomy was the reference standard with pathological complete response (pCR) defined as ypTONO. Diagnostic performance regarding the detection of residual tumour was calculated for gastroscopic biopsies and for EUSFNA without and with MRI.

Results

Nineteen of the 22 patients (86\%) did not achieve pCR after nCRT $(7 \mathrm{ypT}+\mathrm{N}+, 11$ ypT+N0, 1 ypTON+). Biopsies detected residual tumour in 6 of 18 ypT+ patients. After adding MRI, 16 of 18 residual tumours were assessed correctly. EUS-FNA detected 3 out of $8 \mathrm{ypN}+$ patients, while MRI did not improve detection. Overall, adding MRI improved sensitivity for detection of residual tumour to $89 \%$ (17 of 19) from $47 \%$ (9 of 19) with endoscopic biopsies and EUS-FNA only.

\title{
Conclusion
}

In this small study, the detection of residual tumour after nCRT in oesophageal cancer patients was improved by the addition of MRI to gastroscopy and EUS. 


\section{INTRODUCTION}

Oesophageal cancer is the sixth most common cause of cancer death in the world and the ninth most common cancer worldwide'. Patients with locally advanced oesophageal carcinoma are eligible for treatment with curative intent. Neoadjuvant chemoradiotherapy (nCRT) has been shown to improve overall survival compared with surgery alone. Furthermore, in $23 \%$ of the patients with adenocarcinomas and in $49 \%$ of the patients with squamous cell carcinomas, nCRT even results in a pathological complete response ( $P C R$ ) in the resection specimen ${ }^{2}$. As surgery is associated with considerable morbidity, it is questioned if patients with a clinical complete response (CCR) after nCRT could benefit from an organ-sparing approach ${ }^{3}$. However, this approach demands accurate differentiation between complete and incomplete responders after nCRT.

An active surveillance approach for clinical complete responders is currently being investigated by two European research groups ${ }^{4,5}$. In these trials, response assessment after $\mathrm{nCRT}$ is performed with gastroscopy, endoscopic ultrasound (EUS) and 18F-fluorodeoxyglucose positron emission tomography computed tomography (FDG-PET/CT). However, previous studies have shown that in 28 to $55 \%$ of patients assessed as having a CCR with gastroscopic and endosonographic assessment, residual tumour is found in the resection specimen after surgery ${ }^{6-9}$. FDG-PET/CT shows potential in predicting response before the start of treatment and seems important for the detection of interval distant metastases after nCRT ${ }^{10-12}$. However, assessment of FDG-PET/CT has not been able to accurately distinguish residual tumour from radiation oesophagitis and/or fibrosis after $\mathrm{nCRT}^{13,14}$. In rectal cancer, active surveillance by endoscopy and digital rectal examination is supplemented by MRI ${ }^{15,16}$. Due to technological improvement, MRI of the oesophagus is currently also feasible ${ }^{17,18}$. A recent study showed that DW-MRI results in a high sensitivity (90-97\%) for detecting residual tumour in oesophageal cancer patients after $\mathrm{nCRT}{ }^{19}$.

The aim of this study was to investigate whether MRI has added value to gastroscopy with biopsies of the primary tumour area and EUS with fine needle aspirates (FNA) of suspect lymph nodes for the detection of residual tumour after $\mathrm{nCRT}$ in oesophageal cancer patients.

\section{MATERIALS AND METHODS}

\section{Patients}

The study was approved by the local medical ethics committee (NCT02139488) and written informed consent was obtained from all patients. Patients were prospectively enrolled. Inclusion criteria were (1) biopsy-proven locally advanced, non-metastatic oesophageal cancer with or without involvement of the gastro-oesophageal junction, (2) planned curative treatment with nCRT (41.4 Gray in 23 fractions, with weekly administration of carboplatin (AUC 2) and paclitaxel (50 $\mathrm{mg} / \mathrm{m}^{2}$ ) for 5 weeks) followed by oesophagectomy. Exclusion criteria were claustrophobia and metallic implants. 


\section{Response assessment on MRI}

Imaging was acquired on a 1.5-T MRI scanner (Philips Achieva), using Torso-XL (16 channel) receiver coils. The MRI protocol consisted of T2-weighted imaging in transverse and sagittal planes and diffusion-weighted sequences in the transverse plane (b values $0,200,800 \mathrm{~s} / \mathrm{mm}^{2}$ ). A respiratory navigator was placed on the diaphragm and scanning was performed at the end of the expiratory phase ${ }^{20}$. Detailed MRI sequences are provided in Supplementary Table 1. All MRI scans were independently and prospectively analysed by an expert radiologist with 4 years of specific experience interpreting oesophageal MRI (AB). The radiologist reviewed the T2W-and DW-MRI with $b=800 \mathrm{~s} / \mathrm{mm}^{2}$ before and after nCRT. The MRI after nCRT was scored for the presence of residual tumour based on a 5-point confidence level score (CLS): CLS1 = definitely complete response, 2 = probably complete response, 3 = inconclusive, $4=$ probably residual tumour, $5=$ definitely residual tumour 19,21. This score is illustrated in Supplementary Figure 1.

Expert meeting between radiologist and gastroenterologist

After the MRI scan but before gastroscopy+EUS, the MRI scan was discussed by an expert radiologist (A.B.) and expert gastroenterologist (J.VD.). The radiologist showed the images to guide in taking targeted biopsies at locations that remained suspect on MRI after nCRT. The radiologist provided the gastroenterologist with anatomical landmarks for the most suspect areas for residual tumour on MRI, e.g. ventral oesophageal wall $1 \mathrm{~cm}$ above the diaphragm, to direct EUS-guided biopsies.

\section{Response assessment on gastroscopy and EUS}

A baseline gastroscopy+EUS was performed in all patients before the start of $n C R T$. Response assessment on gastroscopy+EUS was performed 6-10 weeks after completion of nCRT and a maximum of 2 weeks after the MRI. All procedures were performed or supervised by an expert gastroenterologist.

During gastroscopy, a minimum of eight bite-on-bite biopsies were taken from areas that were clinically suspect for residual tumour ${ }^{6}$. If no suspect lesions were detected, bite-on-bite biopsies were acquired at random locations in the primary tumour bed. After gastroscopy, a clinical CLS for the presence of residual tumour was assessed (score as aforementioned).

Next, all patients underwent EUS. FNA was performed of suspicious lymph nodes. If the gastroenterologist feared adverse events of performing FNA that could postpone or complicate the upcoming surgery, FNA was not performed and only a CLS of the lymph nodes was determined. In case the radiologist assessed residual tumour in a specific region, the gastroenterologist took EUS-guided biopsies of this area. After EUS, the gastroenterologist assessed the clinical CLS for the presence of residual tumour. If the primary tumour bed could not be passed, this was assessed as CLS4.

After the gastroscopy and EUS, the highest of the confidence level scores was determined as the CLS on gastroscopy+EUS. Biopsy specimens and fine needle aspirates were examined for the 
presence of residual tumour by an expert pathologist.

Adverse events

Serious adverse events (SAE) were defined as death and adverse events that required medication, re-interventions and/or (prolongation of) hospitalisation due to study interventions. All adverse effects of diagnostic procedures performed for study purposes were reported (i.e. the MRI scans and the gastroscopy and EUS re-evaluation).

\section{Surgery}

Depending on tumour and lymph node location, oesophageal resection with lymph node dissection was performed via a transhiatal or transthoracic approach, followed by gastric tube reconstruction and a cervical anastomosis.

\section{Reference standard}

Histopathologic examination of the resection specimens was performed by an experienced gastrointestinal pathologist, in accordance with the ypTNM classification ${ }^{22}$. The ' $y$ ' means that the classification was performed after multimodality therapy and the ' $p$ ' means that it was confirmed during evaluation of histopathology. The tumour bed was completely embedded and tumour regression grading (TRG) of the resected specimen was performed according to Mandard ${ }^{23}$. TRG1 without lymph node metastases (ypTONO) was regarded as PCR, whereas TRG2-5 or TRG1 with lymph node metastases (ypT+N+, ypT+NO or ypTON+) represented residual tumour.

\section{Statistical methods}

Statistical analyses were performed using Statistical Package for the Social Sciences (SPSS, v22). This study was exploratory by nature; therefore, no formal sample size calculation has been performed. Diagnostic performance measures with $95 \%$ confidence intervals $(95 \% \mathrm{Cl}$ ) were computed and receiver operator characteristic $(\mathrm{ROC})$ curve analysis was performed with calculation of the areas under the curve. Before performing the analyses, we chose the cutoff for complete response versus residual tumour between CLS3 and CLS4 (CLS 1/2/3 complete response; CLS $4 / 5$ residual tumour). If the biopsies/FNA resulted in residual tumour, this was assessed a CLS5 (definitely residual tumour) and if the biopsies/FNA were negative, this was regarded a CLS1 (definitely complete response).

First, ROC curve analysis was performed for the detection of ypT+ regarding endoscopic biopsies. In all patients with negative biopsies, the gastroenterologist scores (CLS-gastroscopy or CLS-EUS) or the radiologist score (CLS-MRI) replaced the CLS1 of biopsies only, while patients with positive biopsies remained CLS5. So, a total of four ROC curves were calculated for the detection of ypT+ (biopsy only; biopsy + CLS-gastroscopy; biopsy + CLS-EUS; biopsy + CLS-MRI).

Second, ROC curve analysis was performed for the detection of $y p N+$. The accuracy of FNA only 
was calculated. In all patients with negative FNA, the CLS-EUS and the CLS-MRI for predicting $\mathrm{ypN}+$ replaced the CLS1 of FNA only, while patients with positive FNA remained CLS5. This resulted in three ROC curves for the detection of ypN+ (FNA only; FNA + CLS-EUS; FNA + CLSMRI).

Last, ROC curve analysis was performed for the detection of ypT+/ypTON+. The CLS of biopsies/FNA was combined to result in CLS5 if either biopsies or FNA or both were positive for residual tumour and CLS1 if all were negative. In all patients with negative biopsies/FNA, the gastroenterologists' scores (highest CLS on gastroscopy or EUS) and the CLS-MRI replaced the CLS1 of biopsies/FNA. Thus, three ROC curves were calculated for the detection of ypT+/ypTON+ (biopsies/FNA; biopsies/FNA + CLS-gastroscopy + EUS; biopsies/FNA + CLS-MRI).

\section{RESULTS}

Patient and treatment characteristics

Thirty-one patients signed an informed consent to undergo MRI before and after nCRT and gastroscopy+EUS evaluation after nCRT. Nine patients were excluded for the reasons mentioned in the flowchart (Fig. 1). Eventually, 22 patients were included for the analysis.

Patient and tumour characteristics are shown in Table 1. Twenty patients $(91 \%)$ were diagnosed with adenocarcinoma and 2 patients (9\%) with squamous cell carcinoma. Transhiatal oesophagectomy was performed in 14 patients (64\%) and transthoracic oesophagectomy in 8 patients (36\%). In all 22 patients, the oesophagectomy resulted in a radical resection of the primary tumour bed (RO in $100 \%$ ). The median time interval (interquartile range (IQR)) between the last radiation fraction and surgery was 65 (55-72) days. The median interval (IQR) was 11 (11-18) days between MRI and surgery and 5 (5-12) days between gastroscopy+EUS and surgery. On histopathological assessment, a TRG1 was found in four out of the 22 patients. In three out of these four, no tumour cells were determined in resected lymph nodes (ypTONO), while one patient had ypTON1. The remaining 18 patients had ypT+ with TRG2 in 5/22 (23\%), TRG3 in 9/22 (41\%), TRG4 in 3/22 (14\%) and TRG5 in $1 / 22$ patients (4\%).

\section{Adverse events}

No MRI-related SAEs occurred. One gastroscopy+EUS-related SAE was encountered. This patient presented with fever after the endoscopic procedure and was diagnosed with a probable aspiration pneumonia. The patient was admitted to the hospital for one night and treated with oral antibiotics for 1 week. There was no need to postpone surgery. 
Diagnostic accuracies

The accuracies for the assessment of residual tumour (separately calculated for the detection of ypT+/ypTON+, ypT+ and ypN+) are shown in Table 2. Of all the 22 patients, 19 patients had $y p T+/ y p N+, 18$ patients had ypT+ and 8 patients had ypN+ in the resection specimen.

Assessment of ypT+/ypTON+

Biopsies/FNA correctly detected residual tumour in 9 out of 19 patients (sensitivity $47 \%$ for detecting ypT+/ypTON+; Table 2). ypT+ was detected by biopsies in 6 of 18 patients, and EUS-FNA detected $\mathrm{ypN}+$ in 3 out of 8 patients with lymph node metastases. One of these patients was confirmed ypTON+ after oesophagectomy, whereas the other two patients had ypT+N+ in the resection specimen. The AUC and sensitivity were 0.74 and $47 \%$ for biopsies/FNA only. After adding the MRI, 17 of the 19 patients with residual tumour in the resection specimens were detected, resulting in an AUC and sensitivity of 0.87 and $89 \%$. Figure 2 illustrates a patient with negative outcomes of endoscopic biopsies, CLS2 on visual assessment of gastroscopy+EUS and CLS4 on MRI. In this patient, residual tumour (ypT1bNO, TRG 3) was determined on histopathological examination of the resection specimen with a tumour mass located in the submucosal layer, shown in Fig. 3.

\section{Assessment of ypT+}

For the assessment of ypT+ only, the AUC depending on biopsies was 0.67 and $6 / 18$ patients with residual tumour were correctly detected (sensitivity 33\%). Biopsies + CLS-gastroscopy resulted in an AUC of 0.60 and biopsies + CLS-EUS resulted in an AUC of 0.69. In seven patients, an additional EUS-guided biopsy was performed in the area that was suspect on MRI. One of these biopsies showed residual tumour. Also, in seven patients, an EUS-guided biopsy was taken from the thickest part of the oesophageal wall of which one detected residual tumour. The residual tumour detected by EUS-guided biopsies in these two patients did not affect the accuracies because residual tumour was already confirmed in the regular bite-on-bite biopsies. Lastly, combining the biopsies with the CLS-MRI led to an AUC of 0.79 with a sensitivity of $89 \%$ ( $16 / 18$ patients with ypT+ were correctly detected).

\section{Assessment of ypN+}

In 9 out of the 22 patients, FNA of suspect lymph nodes was performed. The AUC for FNA to predict ypN+ was 0.69 with a sensitivity of $38 \%$ ( 3 out of 8 patients with ypN+ were detected). This AUC increased to 0.80 and the sensitivity increased to $88 \%$ after adding the gastroenterologists' CLS on EUS (7 of 8 patients with ypN+ were correctly assessed). Adding MRI to FNA for predicting $\mathrm{ypN}+$ resulted in an AUC of 0.73 and the sensitivity remained $38 \%$. 
31 signed informed consent

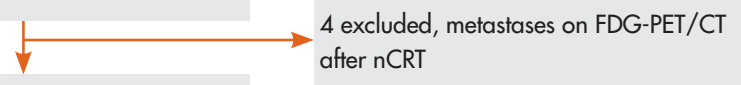

27 eligible for response assessment on MRI

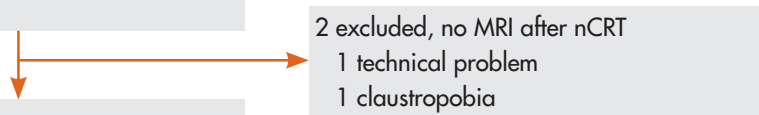

25 eligible for response assessment on endoscopy+EUS

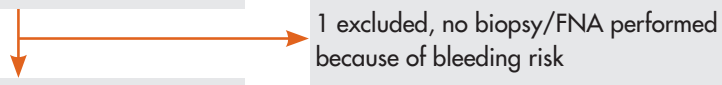

24 underwent response assessment on MRI and endoscopy+EUS

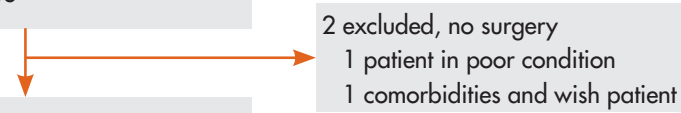

22 inluded for analysis

Figure 1. Flowchart of the study

FDG-PET/CT = 18F fluorodeoxyglucose positron emission tomography; $\mathrm{nCRT}=$ neoadjuvant chemoradiotherapy; $\mathrm{MRI}=$ magnetic resonance imaging; $\mathrm{EUS}$ = endosonographic ultrasound; FNA = fine needle aspiration 
Table 1. Patient and tumour characteristics

\begin{tabular}{|c|c|}
\hline Characteristic & $\mathrm{n}(\%)$ \\
\hline Gender: male & $18(81 \%)$ \\
\hline Median age (years) a & $63(59-70)$ \\
\hline $\begin{array}{l}\text { Tumour location } \\
\text { Upper oesophageal } \\
\text { Middle oesophageal } \\
\text { Lower oesophageal } \\
\text { Gastro-oesophageal junction }\end{array}$ & $\begin{array}{l}1(4.5 \%) \\
3(13.6 \%) \\
9(40.9 \%) \\
9(50.9 \%)\end{array}$ \\
\hline $\begin{array}{l}\text { Histological tumour type } \\
\text { Adenocarcinoma } \\
\text { Laurén classification for adenocarcinomas: } \\
\text { Intestinal } \\
\text { Diffuse } \\
\text { Mixed } \\
\text { Squamous cell carcinoma }\end{array}$ & $\begin{array}{l}20(91 \%) \\
14 / 20(70 \%) \\
4 / 20(20 \%) \\
2 / 20(10 \%) \\
2(9 \%)\end{array}$ \\
\hline $\begin{array}{l}\text { Grade of differentiation } \\
\text { Well differentiated } \\
\text { Moderately differentiated } \\
\text { Poorly differentiated } \\
\text { Undifferentiated }\end{array}$ & $\begin{array}{l}0(0 \%) \\
10(45 \%) \\
7(32 \%) \\
5(23 \%)\end{array}$ \\
\hline $\begin{array}{l}\text { Clinical T-stage } \\
\text { cT1 } \\
\text { cT2 } \\
\text { cT3 }\end{array}$ & $\begin{array}{l}0 \\
7 \\
15 \\
0\end{array}$ \\
\hline $\begin{array}{l}\text { Clinical } \mathrm{N} \text {-stage }{ }^{b} \\
\text { cN0 } \\
\text { cN1 } \\
\text { cN2 } \\
\text { cN3 }\end{array}$ & $\begin{array}{l}7 \\
7 \\
7 \\
1\end{array}$ \\
\hline $\begin{array}{l}\text { Pathology after surgeryc } \\
\text { TRG } 1 \text { and NO: ypTONO } \\
\text { TRG } 1 \text { and N1: ypTON+ } \\
\text { TRG 2, or ypTON+ } \\
\text { TRG } 3 \\
\text { TRG } 4 \\
\text { TRG } 5 \\
\end{array}$ & $\begin{array}{l}3(14 \%) \\
1(4.5 \%) \\
5(23 \%) \\
9(40 \%) \\
3(14 \%) \\
1(4.5 \%)\end{array}$ \\
\hline $\begin{array}{l}\text { Time intervals (days) a } \\
\text { End of nCRT until esophagectomy } \\
\text { End of nCRT until MRI post-nCRT } \\
\text { End of nCRT until gastroscopy+EUS } \\
\text { MRI post-nCRT until gastroscopy+EUS } \\
\text { MRI post-nCRT until surgery } \\
\text { Gastroscopy+EUS until surgery }\end{array}$ & $\begin{array}{l}65(55-72) \\
49(45-57) \\
55(50-65) \\
6(5-7) \\
11(11-18) \\
5(5-12)\end{array}$ \\
\hline $\begin{array}{l}\text { a Data are presented in medians and data in parent } \\
\text { b Clinical staging of the oesophageal tumor and lym } \\
\text { c The tumor regression grade of the primary tumor } \\
n \text {, number patients; TRG, tumor regression grade; } n\end{array}$ & $\begin{array}{l}\text { interquartile range } \\
\text { g to the } 7 \text { th edition of the TNM classification }{ }^{22} \\
\text { ccording to Mandard }{ }^{23} \\
\text { eemoradiotherapy; MRI, magnetic resonance imaging }\end{array}$ \\
\hline
\end{tabular}


Table 2. Diagnostic accuracies for the detection of ypT+/ypTON+ (versus ypTONO), ypT+ (versus ypTO) and ypN+ (versus ypNO)

\begin{tabular}{|c|c|c|c|c|c|c|}
\hline $\begin{array}{l}\text { Detection of } \\
\text { ypT+/ypTON+ }\end{array}$ & $\begin{array}{l}\text { AUC } \\
(95 \% \mathrm{CI})\end{array}$ & $\begin{array}{l}\text { Accuracy\%, } \\
\mathrm{n}\end{array}$ & $\begin{array}{l}\text { Sensitivity\% } \\
(95 \% \mathrm{Cl}), \mathrm{n}\end{array}$ & $\begin{array}{l}\text { Specificity\% } \\
(95 \% \text { Cl), n }\end{array}$ & $\begin{array}{l}\text { NPV\% } \\
(95 \% \mathrm{Cl}), \mathrm{n}\end{array}$ & $\begin{array}{l}\text { PPV\% } \\
(95 \% \mathrm{Cl}), \mathrm{n}\end{array}$ \\
\hline $\begin{array}{l}\text { Biopsies/FNA } \\
\text { only }\end{array}$ & $\begin{array}{l}0.74 \\
(0.50-0.97)\end{array}$ & $55,12 / 22$ & $\begin{array}{l}47(25-71) \\
9 / 19\end{array}$ & $\begin{array}{l}100(31- \\
100), 3 / 3\end{array}$ & $\begin{array}{l}23(6-54), \\
3 / 13\end{array}$ & $\begin{array}{l}100(63- \\
100), 9 / 9\end{array}$ \\
\hline $\begin{array}{l}\text { Biopsies/ } \\
\text { FNA + CLS }\end{array}$ & $\begin{array}{l}0.71 \\
(0.46-0.96)\end{array}$ & $68,15 / 22$ & $\begin{array}{l}74(49-90) \\
14 / 19\end{array}$ & $\begin{array}{l}33(2-87), \\
1 / 3\end{array}$ & $\begin{array}{l}17(1-64), \\
1 / 6\end{array}$ & $\begin{array}{l}88 \text { (60-98), } \\
14 / 16\end{array}$ \\
\hline $\begin{array}{l}\text { Biopsies/FNA + } \\
\text { CLS MRI }\end{array}$ & $\begin{array}{l}0.87 \\
(0.68-1.00)\end{array}$ & $86,19 / 22$ & $\begin{array}{l}89(65-98) \\
17 / 19\end{array}$ & $\begin{array}{l}67(13-98), \\
2 / 3\end{array}$ & $\begin{array}{l}50(9-91), \\
2 / 4\end{array}$ & $\begin{array}{l}94(71-100), \\
17 / 18\end{array}$ \\
\hline \multicolumn{7}{|l|}{ Detection of ypT+ } \\
\hline Biopsies only & $\begin{array}{l}0.67 \\
(0.41-0.92)\end{array}$ & $45,10 / 22$ & $\begin{array}{l}33(14-59), \\
6 / 18\end{array}$ & $\begin{array}{l}100(40- \\
100), 4 / 4\end{array}$ & $\begin{array}{l}25(8-53), \\
4 / 16\end{array}$ & $\begin{array}{l}100(52- \\
100), 6 / 6\end{array}$ \\
\hline $\begin{array}{l}\text { Biopsies + CLS } \\
\text { gastroscopy }\end{array}$ & $\begin{array}{l}0.60 \\
(0.34-0.87)\end{array}$ & $59,13 / 22$ & $\begin{array}{l}67(41-86) \\
12 / 18\end{array}$ & $\begin{array}{l}25(1-78), \\
1 / 4\end{array}$ & $\begin{array}{l}14(1-58), \\
1 / 7\end{array}$ & $\begin{array}{l}80(51-95) \\
12 / 15\end{array}$ \\
\hline $\begin{array}{l}\text { Biopsies + CLS } \\
\text { EUS }\end{array}$ & $\begin{array}{l}0.69 \\
(0.45-0.93)\end{array}$ & $50,11 / 22$ & $\begin{array}{l}56(31-78) \\
10 / 18\end{array}$ & $\begin{array}{l}25(1-78), \\
1 / 4\end{array}$ & $\begin{array}{l}11(1-49), \\
1 / 9\end{array}$ & $\begin{array}{l}77(46-94) \\
10 / 13\end{array}$ \\
\hline $\begin{array}{l}\text { Biopsies + CLS } \\
\text { MRI }\end{array}$ & $\begin{array}{l}0.79 \\
(0.57-100) \\
\end{array}$ & $82,18 / 22$ & $\begin{array}{l}89(64-98), \\
16 / 18\end{array}$ & $\begin{array}{l}50(9-91), \\
2 / 4\end{array}$ & $\begin{array}{l}50(9-91), \\
2 / 4\end{array}$ & $\begin{array}{l}89(64-98) \\
16 / 18\end{array}$ \\
\hline \multicolumn{7}{|c|}{ Detection of ypN+ } \\
\hline FNA only ${ }^{a}$ & $\begin{array}{l}0.69 \\
(0.43 .0 .94)\end{array}$ & $77,17 / 22$ & $\begin{array}{l}38(10-74) \\
3 / 8\end{array}$ & $\begin{array}{l}100(73- \\
100), 14 / 14\end{array}$ & $\begin{array}{l}74(49-90), \\
14 / 19\end{array}$ & $\begin{array}{l}100(31- \\
100), 3 / 3\end{array}$ \\
\hline FNA + CLS EUS & $\begin{array}{l}0.80 \\
(0.60-100)\end{array}$ & $68,15 / 22$ & $\begin{array}{l}88(47-99), \\
7 / 8\end{array}$ & $\begin{array}{l}57(30-81), \\
8 / 14\end{array}$ & $\begin{array}{l}89(51-99), \\
8 / 9\end{array}$ & $\begin{array}{l}54(26-80) \\
7 / 13\end{array}$ \\
\hline $\mathrm{FNA}+\mathrm{CLS}$ MRI & $\begin{array}{l}0.73 \\
(0.51-0.96)\end{array}$ & $73,16 / 22$ & $\begin{array}{l}38(10-74) \\
3 / 8\end{array}$ & $\begin{array}{l}93(64-100), \\
13 / 14\end{array}$ & $\begin{array}{l}72(46-89), \\
13 / 18\end{array}$ & $\begin{array}{l}75(22-99) \\
3 / 4\end{array}$ \\
\hline
\end{tabular}

ypTON+/ypT+/ypN+, pathology of the specimen after esophagectomy according to the 7th edition of the TNM classification ${ }^{19}$; MRI, magnetic resonance imaging; CLS, confidence level score; NPV, negative predictive value; PPV, positive predictive value; $95 \% \mathrm{Cl}, 95 \%$ confidence interval; EUS, endoscopic ultrasonography; FNA, fine needle aspiration; $n$, number of patients a If FNA was not performed, this was scored as a negative FNA outcome 

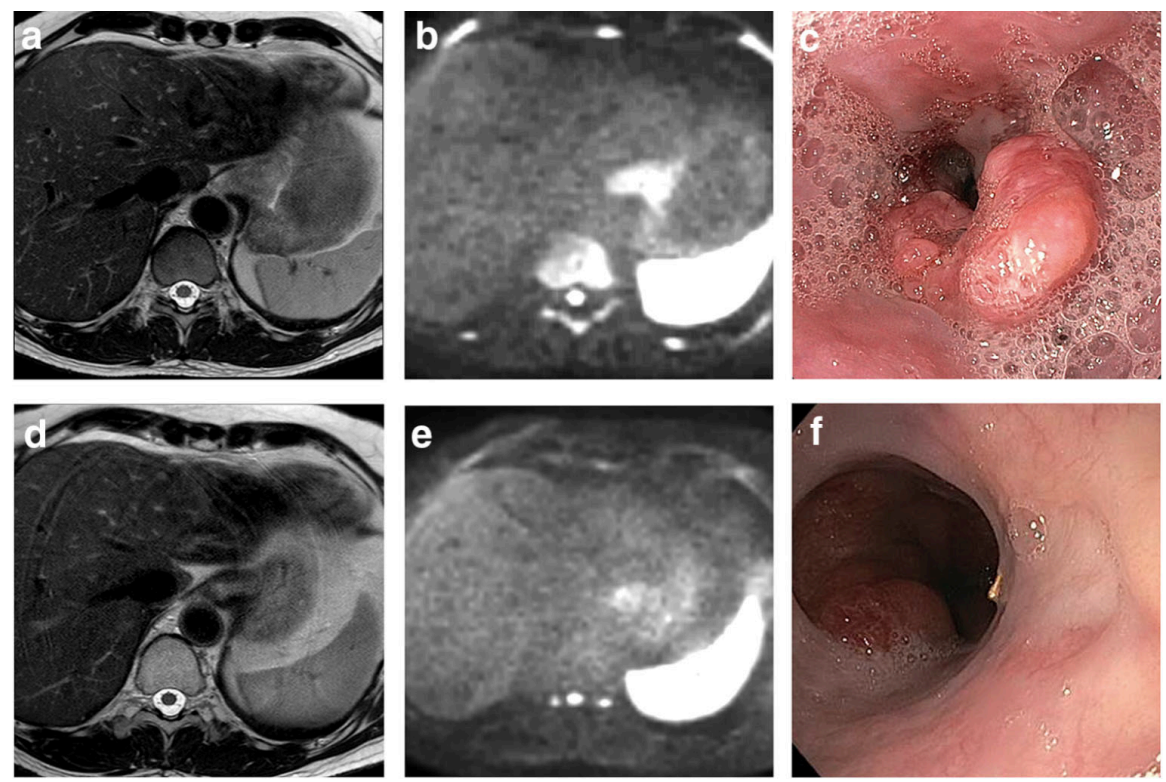

Figure 2. Patient with false negative endoscopic biopsies; false negative visual assessment on endoscopy; true positive MRI assessment and residual tumour confirmed at histopathological examination of the resection specimen after oesophagectomy.

A 52-year-old male patient with a cT3NO distal adenocarcinoma of the oesophagus, with residual tumour after neoadjuvant chemoradiotherapy and oesophagectomy (shown in Fig. 3). The T2weighted transverse (a) image before chemoradiotherapy showed a thickened oesophageal wall, accompanied by hyperintense signal on diffusion-weighted imaging (b) and an oesophageal tumour on endoscopy (c). The T2-weighted transverse (d) image after chemoradiotherapy showed a shrinkage of the wall with considerable hypointense signal, reflecting fibrosis. The diffusionweighted MRI after neoadjuvant treatment (e) still showed hyperintense signal in the primary tumour area which was suspect for residual tumour and assigned as a confidence level score 4. On endoscopy after neoadjuvant therapy ( $f$ ), the primary tumour area was not suspect for residual tumour (confidence level score 2) and bite-on-bite biopsies of the primary tumour bed were negative. 
a

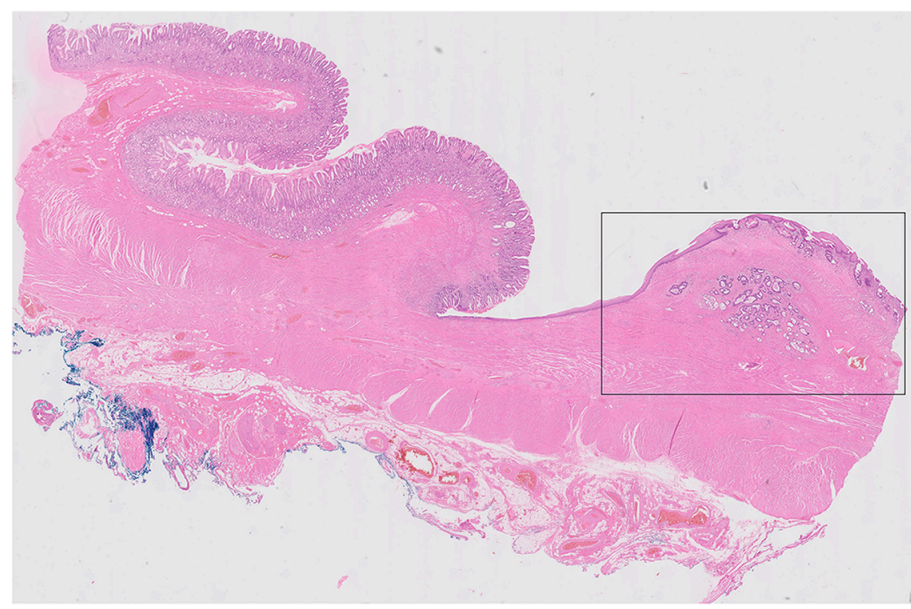

b

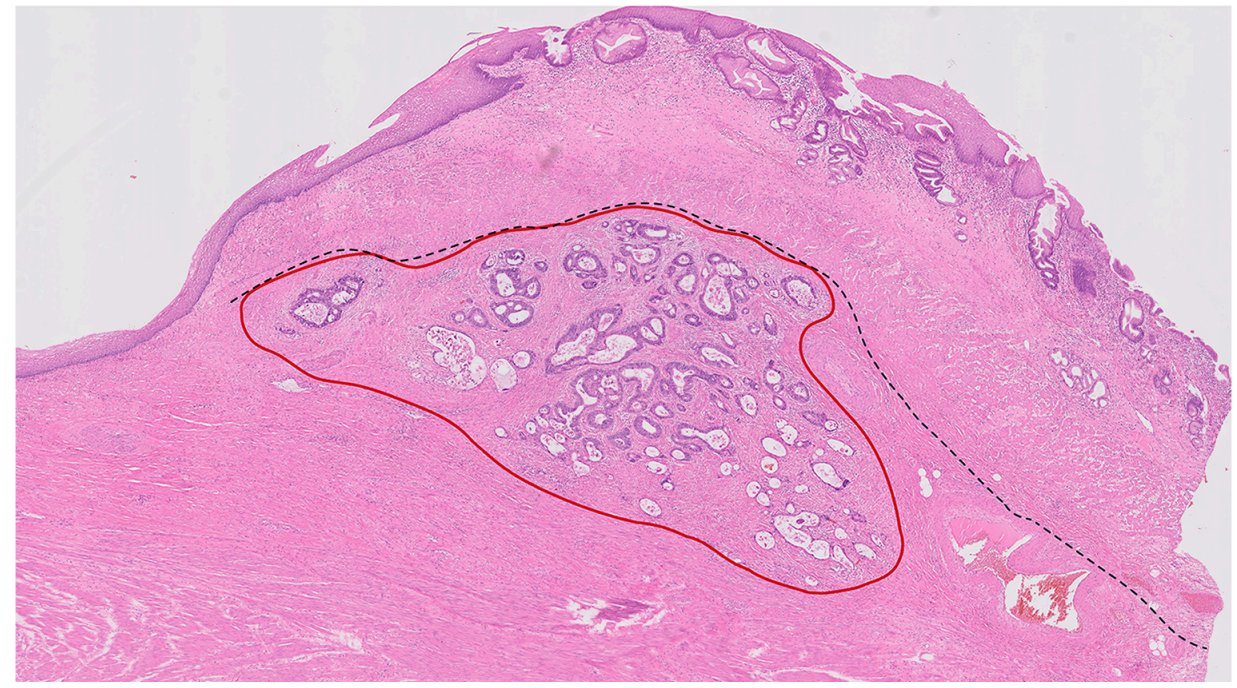

Figure 3. Histopathological examination of the resection specimen of the patient outlined in Fig. 2 showed a tumour regression grade 3, ypT1bNO. Histopathological examination of the resection specimen of the patient outlined in Fig. 2.

a An overview of the resected specimen with the gastric folds on the left side and the gastrooesophageal junction on the right side. The residual tumour cells are located in the black square.

b Represents the black square with a black dashed line indicating the separation between the mucosal and submucosal layer. The residual tumour cells are designated by the red line in the submucosal layer. 


\section{DISCUSSION}

This study is the first to evaluate whether MRI has added value to gastroscopy and EUS in the assessment of residual tumour after nCRT for oesophageal cancer. In this small sample, the addition of MRI assessment to pathology results of biopsies/FNA acquired by gastroscopy and EUS resulted in a notable increase in accuracy and sensitivity for the detection of residual tumour. This implies that the considerable chance of missing residual tumour after nCRT with only gastroscopy and EUS decreases after adding MRI. However, even in the current small study, the risk of false positive MRI was demonstrated with one out of three complete responders incorrectly assessed as residual tumour on MRI.

Currently two research groups are evaluating an active surveillance approach for clinical complete responders ${ }^{4,5}$. A precursor study assessed the detection of residual tumour by gastroscopy which was followed by a second gastroscopy with EUS in patients with negative biopsies. This resulted in a sensitivity of $77 \%$ and specificity of $72 \%$ for detecting residual tumour after $n$ CRT by using bite-on-bite biopsies and $\mathrm{FNA}^{6}$. The sensitivity for detecting residual tumour of gastroscopy and EUS could be improved to $89 \%$ in the current study by adding a noninvasive MRI assessment, which therefore seems to yield better results than the addition of a second gastroscopy with EUS as was done in the aforementioned precursor study ${ }^{6}$. If surgery is to be safely omitted, detection of residual tumour needs to be optimal7,9. Therefore, the addition of MRI to gastroscopy and EUS seems of value in the implementation of an active surveillance approach. Furthermore, in rectal cancer, the combination of rectoscopy and MRI has shown to be effective in assessing the response to chemoradiotherapy and is now seen as a valuable tool in an active surveillance strategy ${ }^{15,16}$. Another modality that has been examined to improve the sensitivity of endoscopic assessment for detecting residual tumour is FDG-PET/CT. Two studies found sensitivities/specificities of $24 \% / 83 \%$ $\left(n=138,{ }^{24}\right)$ and of $30 \% / 97 \%\left(n=284,{ }^{25}\right)$ based on endoscopic assessment combined with FDG-PET/CT. Although these studies only incorporate endoscopic biopsies (without EUS-FNA), the poor sensitivities after adding FDG-PET/CT reflect the inability of FDG-PET/CT to assess response after $\mathrm{nCRT}$ for oesophageal cancer. Potential reasons for this inability are the inability to differentiate between radiation-induced oesophagitis and residual tumour on FDG-PET/CT and a detection threshold that is too high to pick up small residual tumours ${ }^{26}$. Based on our (small) cohort of patients, MRI seems to outperform FDG-PET/CT in assessing the response of the primary oesophageal tumour after nCRT, especially in terms of sensitivity for residual tumour detection.

For the detection of ypT+, our study showed that gastroscopy with biopsies resulted in a sensitivity and specificity of $33 \%$ and $100 \%$. These results are in line with a systematic review which reported pooled estimates for sensitivity and specificity of endoscopic biopsies after nCRT for assessment of ypT+ of 35\% and $91 \% 27$. The addition of the gastroenterologists' CLS on gastroscopy to biopsy alone did not result in an improvement of predicting ypT+. This reflects the difficulty of visual differentiation of residual tumour and inflammatory/reactive tissue on gastroscopy. Prolonging the 
interval between the end of $\mathrm{nCRT}$ and gastroscopy may reduce the presence of radiation-induced oesophagitis and ulcers and thereby improve the ability to differentiate between residual tumour and complete response on gastroscopy. Yet, in patients with residual tumour after nCRT and surgery, the mucosa is not involved in $28 \%$ of the cases ${ }^{28}$. Therefore, visual assessment on gastroscopy may not be an ideal evaluation strategy. On EUS, the oesophageal wall can be visualised and a recent study showed that residual wall thickness was associated with TRG3-4 residual disease ${ }^{29}$. However, the visual differentiation between tumour, fibrosis and inflammation after nCRT remains difficult ${ }^{30}$. This is in line with our finding that addition of the CLS on EUS did not result in an AUC increase for detecting yрT+. Conversely, MRI both visualises the complete wall and allows the differentiation between tumour and fibrosis with diffusion weighting. The expert meeting between the radiologist and the gastroenterologist resulted in the performance of EUS-guided biopsies which did not affect the accuracy to detect residual tumour in our cohort. However, the addition of the assessment on MRI to the endoscopic biopsies resulted in a notable AUC increase which encourages the combination of biopsies/FNA and MRI for response assessment in oesophageal cancer.

For the detection of $\mathrm{ypN}+$, the EUS-FNA resulted in an AUC of 0.69 with a sensitivity of only $38 \%$. An explanation for the moderate accuracy of EUS-FNA is that FNA was not performed in 5 out of 14 patients with suspect lymph nodes because the gastroenterologist feared adverse events of performing FNA that could postpone or complicate the upcoming surgery. This is a limitation of this study concerning the EUS evaluation. These results, however, are in line with a previous study which reported a low sensitivity of $26 \%$ for EUS-FNA after $n C R T^{31}$. Risk for (mediastinal) bleeding during the FNA procedure withheld the gastroenterologist from aggressive FNA of all suspect lymph nodes. More aggressive application of FNA is desirable in active surveillance strategies because of major clinical consequences if lymph node metastases are missed. After adding the gastroenterologists' CLS on EUS, the AUC improved from 0.69 to 0.80 and also the sensitivity improved from 38 to $88 \%$. This encourages FNA of all suspect lymph nodes when considering an active surveillance approach for patients with a clinical complete response. The sensitivity of the current study to predict residual tumour positive nodes was higher than that of a previous study which assessed clinical $\mathrm{N}$ staging by the gastroenterologist based on EUS in 73 patients (sensitivity $\left.67 \%,{ }^{30}\right)$. The addition of MRI to EUS-FNA in our study did not improve the AUC for detection of $y p N+$ disease (AUC 0.73) and the sensitivity was still low (38\%). The poor sensitivity for detection of $y p N+$ on MRI may be partially explained by the fact that on MRI not all locoregional lymph nodes could be included in the field of view due to scan time restrictions. The assessment of locoregional lymph node response on imaging in oesophageal cancer remains a challenge and is subject of ongoing research ${ }^{32-34}$.

Some limitations must be considered. First, although this is the first study combining gastroscopy, EUS and MRI to detect residual tumour after nCRT, the sample size is small. Indeed, the number of patients with ypTONO/TRG1 is very limited $(n=3)$ resulting in a specificity with large confidence intervals. Second, it should be noted that the assessment by the gastroenterologist may be biased by 
the radiologists' opinion of response on MRI that was shared during the expert meeting. However, as the radiologists' accuracy was high, in that case, a high accuracy of detecting residual tumour by the gastroenterologists' visual assessment would be expected, which was not found in this study. Lastly, as discussed above, FNA was not performed in all patients with suspect lymph nodes.

In conclusion, in this study, the addition of MRI to the gastroscopic and endosonographic assessment after $\mathrm{nCRT}$ in oesophageal cancer patients improved the detection of residual tumour. Larger studies on this combination are warranted and a multimodal assessment strategy including gastroscopy, EUS and MRI may allow safe implementation of active surveillance in oesophageal cancer patients after chemoradiotherapy. 


\section{REFERENCES}

1. Bray F, Ferlay J, Soeriomataram I, Siegel RL, Torre LA, Jemal A. Global cancer statistics 2018: GLOBOCAN estimates of incidence and mortality worldwide for 36 cancers in 185 countries. CA: a cancer journal for clinicians 2018;68:394-424.

2. van Hagen $P$, Hulshof $M C$, van Lanschot $J J$, et al. Preoperative chemoradiotherapy for esophageal or junctional cancer. N Engl J Med 2012;366:2074-84.

3. van der Wilk BJ, Eyck BM, Spaander MCW, et al. Towards an Organ-Sparing Approach for Locally Advanced Esophageal Cancer. Dig Surg 2018:1-8.

4. clinicaltrials.gov [internet]. Centre Hospitalier Universitaire Dijon: Comparison of Systematic Surgery Versus Surveillance and Rescue Surgery in Operable Oesophageal Cancer With a Complete Clinical Response to Radiochemotherapy (Esostrate) NCTO2551458 2015 [updated 1/12/16]. Available: https:// clinicaltrials.gov/show/NCT02551458.

5. Noordman BJ, Wijnhoven BPL, Lagarde SM, et al. Neoadjuvant chemoradiotherapy plus surgery versus active surveillance for oesophageal cancer: a stepped-wedge cluster randomised trial. BMC Cancer 2018;18:142.

6. Noordman BJ, Spaander MCW, Valkema $\mathrm{R}$, et al. Detection of residual disease after neoadjuvant chemoradiotherapy for oesophageal cancer (preSANO): a prospective multicentre, diagnostic cohort study. Lancet Oncol 2018;19:965-74.

7. Castoro C, Scarpa M, Cagol M, et al. Complete clinical response after neoadjuvant chemoradiotherapy for squamous cell cancer of the thoracic oesophagus: is surgery always necessary? J Gastrointest Surg 2013;17:137581.

8. Furlong $H$, Bass $G$, Breathnach $O, O^{\prime}$ Neill $B$, Leen E, Walsh TN. Targeting therapy for esophageal cancer in patients aged 70 and over. J Geriatr Oncol 2013;4:107-13.

9. Ohkura $Y$, Shindoh J, Ueno $M$, lizuka $T$, Udagawa $\mathrm{H}$. Comparison of Outcome of Esophagectomy Versus Nonsurgical Treatment for Resectable Esophageal Cancer with Clinical
Complete Response to Neoadjuvant Therapy. Ann Surg Oncol 2018;25:2428-33.

10. Findlay $J M$, Gillies RS, Franklin $J M$, et al. Restaging oesophageal cancer after neoadjuvant therapy with (18)F-FDG PET-CT: identifying interval metastases and predicting incurable disease at surgery. Eur Radiol 2016;26:3519-33.

11. Kroese TE, Goense L, van Hillegersberg R, et al. Detection of distant interval metastases after neoadjuvant therapy for esophageal cancer with 18F-FDG PET(/CT): a systematic review and meta-analysis. Dis Esophagus 2018;31 .

12. Foley KG, Hills RK, Berthon B, et al. Development and validation of a prognostic model incorporating texture analysis derived from standardised segmentation of PET in patients with oesophageal cancer. Eur Radiol 2018;28:428-36.

13. de Gouw D, Klarenbeek BR, Driessen M, et al. Detecting Pathological Complete Response in Esophageal Cancer after Neoadjuvant Therapy Based on Imaging Techniques: A Diagnostic Systematic Review and Meta-Analysis. J Thorac Oncol 2019;14:1156-71

14. Eyck BM, Onstenk BD, Noordman BJ, et al. Accuracy of Detecting Residual Disease After Neoadjuvant Chemoradiotherapy for Esophageal Cancer: A Systematic Review and Meta-analysis. Ann Surg 2019.

15. Maas $M$, Lambregts DM, Nelemans PJ, et al. Assessment of Clinical Complete Response After Chemoradiation for Rectal Cancer with Digital Rectal Examination, Endoscopy, and MRI: Selection for Organ-Saving Treatment. Ann Surg Oncol 2015;22:3873-80.

16. van der Valk MJM, Hilling DE, Bastiaannet $E$, et al. Long-term outcomes of clinical complete responders after neoadjuvant treatment for rectal cancer in the International Watch \& Wait Database (IWWD): an international multicentre registry study. Lancet 2018;391:2537-45.

17. Yip C, Cook GJ, Landau DB, Davies A, Goh V. Performance of different imaging modalities in assessment of response to neoadjuvant therapy in primary esophageal cancer. Dis Esophagus 2016;29:116-30. 
18. Yamada I, Miyasaka N, Hikishima K, et al. Ultra-high-resolution MR imaging of esophageal carcinoma at ultra-high field strength (7.OT) ex vivo: correlation with histopathologic findings. Magn Reson Imaging 2015;33:413-9.

19. Vollenbrock SE, Voncken FEM, van Dieren $J M$, et al. Diagnostic performance of MRI for assessment of response to neoadjuvant chemoradiotherapy in oesophageal cancer. $\mathrm{Br}$ J Surg 2019;106:596-605.

20. Lever FM, Lips IM, Crijns SP, et al. Quantification of esophageal tumor motion on cine-magnetic resonance imaging. Int J Radiat Oncol Biol Phys 2014;88:419-24.

21. Lambregts DM, Vandecaveye V, Barbaro B, et al. Diffusion-weighted MRI for selection of complete responders after chemoradiation for locally advanced rectal cancer: a multicenter study. Ann Surg Oncol $2011 ; 18: 2224-31$.

22. Rice TW, Blackstone EH, Rusch WW. 7th edition of the AJCC Cancer Staging Manual: esophagus and esophagogastric junction. Ann Surg Oncol 2010;17:1721-4

23. Mandard AM, Dalibard F, Mandard JC, et al Pathologic assessment of tumor regression after preoperative chemoradiotherapy of esophageal carcinoma. Clinicopathologic correlations. Cancer 1994;73:2680-6.

24. Heneghan HM, Donohoe C, Elliot J, et al. Can CT-PET and Endoscopic Assessment PostNeoadjuvant Chemoradiotherapy Predict Residual Disease in Esophageal Cancer? Ann Surg 2016;264:831-8.

25. Cheedella NK, Suzuki A, Xiao L, et al. Association between clinical complete response and pathological complete response after preoperative chemoradiation in patients with gastroesophageal cancer: analysis in a large cohort. Ann Oncol 2013;24:1262-6.

26. Kitajima $K$, Nakajo $M$, Kaida $H$, et al. Present and future roles of FDG-PET/CT imaging in the management of gastrointestinal cancer: an update. Nagoya J Med Sci 2017;79:527-43.

27. van Rossum PS, van Lier $A L$, van Vulpen $M$, et al. Diffusion-weighted magnetic resonance imaging for the prediction of pathologic response to neoadjuvant chemoradiotherapy in esophageal cancer. Radiother Oncol 2015;115:163-70.
28. Shapiro J, ten Kate FJ, van Hagen $P$, Biermann K, Wijnhoven BP, van Lanschot JJ. Residual esophageal cancer after neoadjuvant chemoradiotherapy frequently involves the mucosa and submucosa. Ann Surg 2013;258:678-88.

29. van der Bogt RD, Noordman BJ, Krishnadath KK, et al. Endoscopic ultrasound measurements for detection of residual disease after neoadjuvant chemoradiotherapy for esophageal cancer. Endoscopy 2019;51:326-32.

30. Griffin JM, Reed CE, Denlinger CE. Utility of restaging endoscopic ultrasound after neoadjuvant therapy for esophageal cancer. Ann Thorac Surg 2012;93:1855-9.

31. Eloubeidi MA, Cerfolio RJ, Bryant AS, Varadarajulu S. Efficacy of endoscopic ultrasound in patients with esophageal cancer predicted to have NO disease. Eur J Cardiothorac Surg 2011 ; 40:636-41.

32. Findlay $J M$, Dickson $E$, Fiorani $C$, et al. Temporal validation of metabolic nodal response of esophageal cancer to neoadjuvant chemotherapy as an independent predictor of unresectable disease, survival, and recurrence. Eur Radiol 2019.

33. Qu J, Shen C, Qin J, et al. The MR radiomic signature can predict preoperative lymph node metastasis in patients with esophageal cancer. Eur Radiol 2019;29:906-14.

34. Tan X, Ma Z, Yan L, Ye W, Liu Z, Liang C. Radiomics nomogram outperforms size criteria in discriminating lymph node metastasis in resectable esophageal squamous cell carcinoma. Eur Radiol 2019;29:392-400. 


\section{SUPPLEMENTARY FILES}

Suppl. Table 1. MRI sequence parameters

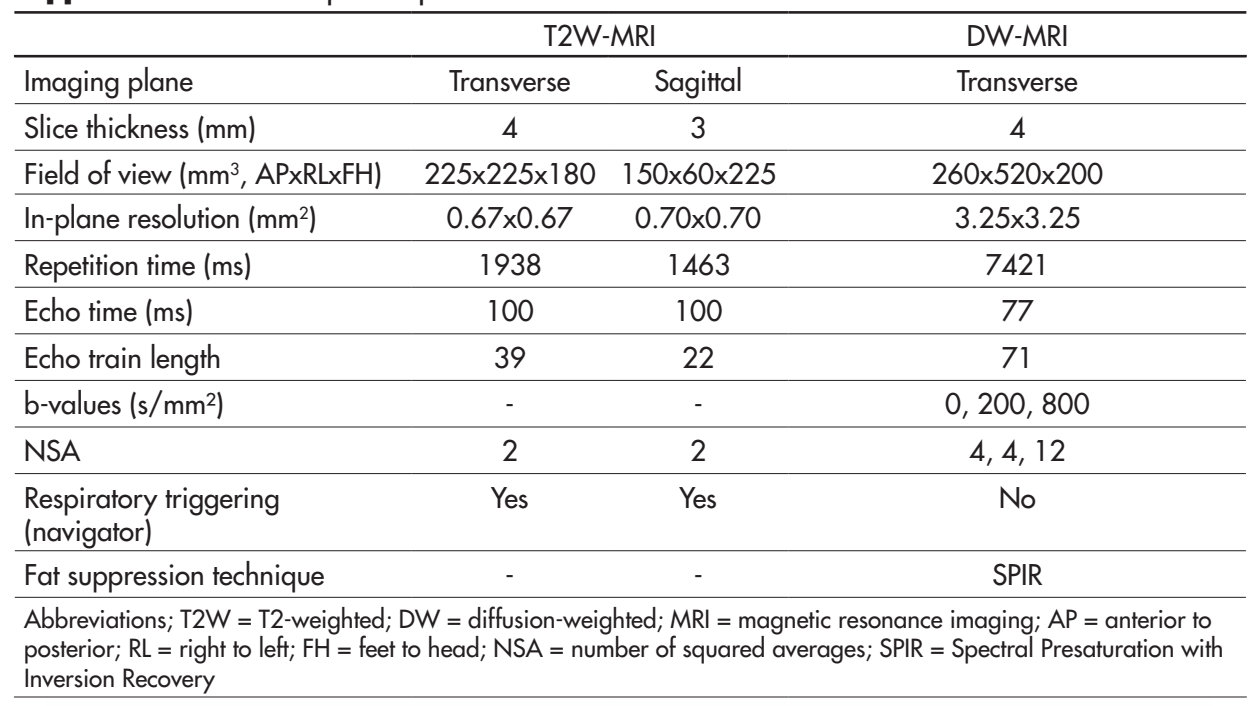

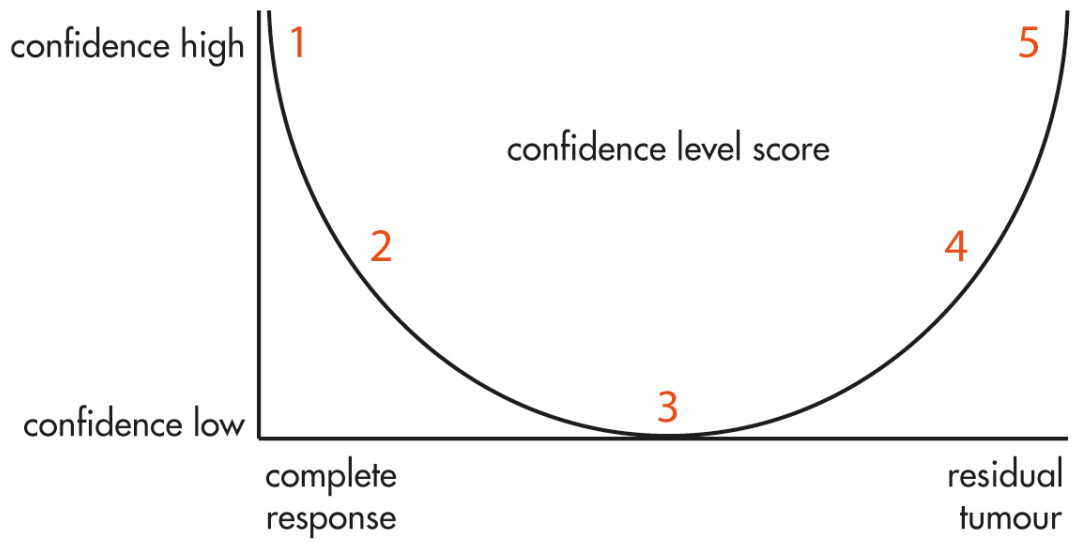

Suppl. Figure 1. Confidence level score

This Figure illustrates the confidence of the readers from low to high (y-axis) and the suspicion of residual disease from low to high ( $x$-axis). 
ADDED VALUE OF MRI TO ENDOSCOPIC AND ENDOSONOGRAPHIC RESPONSE ASSESSMENT 


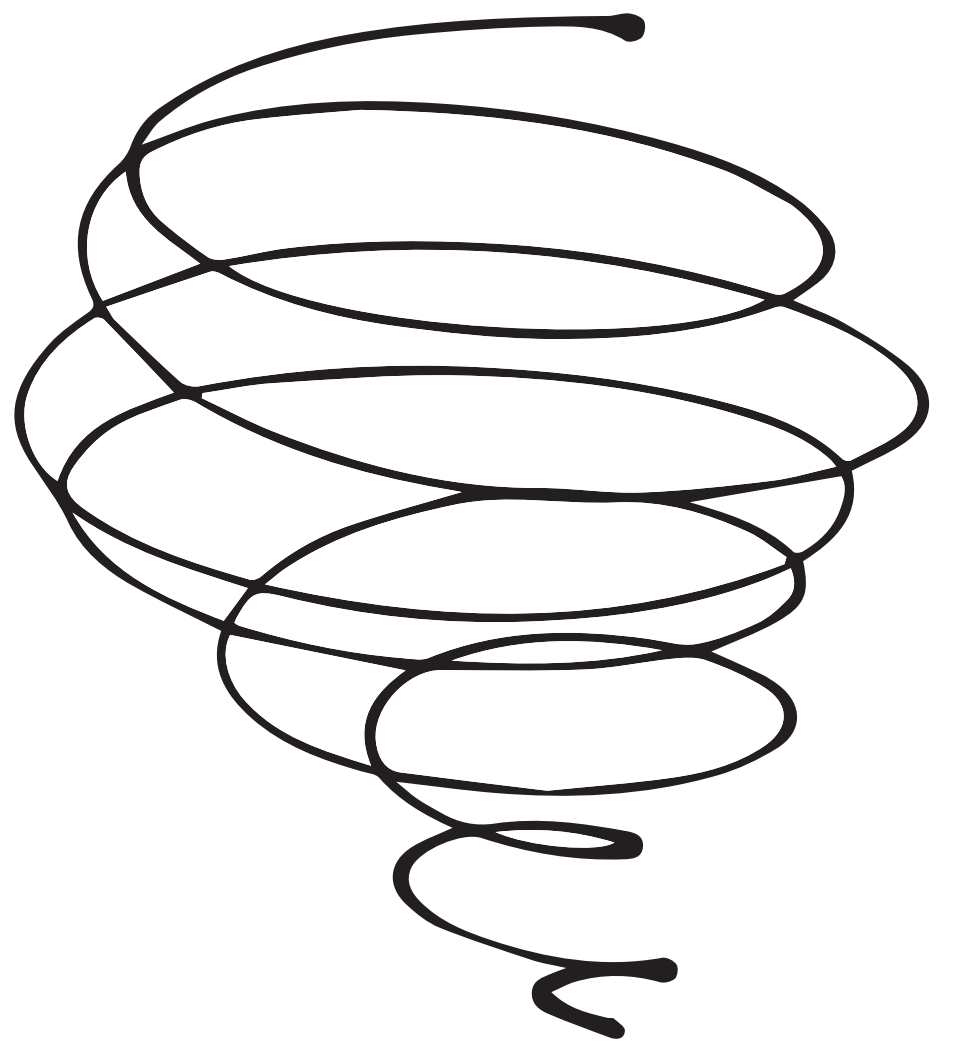




\section{CHAPTER 6}

\section{Gross tumor delineation in esophageal cancer on MRI compared with 18F-FDG-PET/CT}

Advances in Radiation Oncology; Apr 2019 (Volume 4, Issue 4, p596-604)

Sophie E. Vollenbrock* Marlies E. Nowee*

Francine E.M. Voncken Alexis N.T.J. Kotte Lucas Goense

Peter S.N. van Rossum Astrid L.H.M.W. van Lier Stijn W. Heijmink Annemarieke Bartels-Rutten Frank J. Wessels Berthe M.P. Aleman Luc Dewit Linda G.W. Kerkmeijer Edwin P.M. Jansen Martiin Intven Irene M. Lips Gert J. Meijer Jasper Nijkamp

${ }^{*}$ Contributed equally 


\begin{abstract}
Purpose

Current delineation of the gross tumor volume (GTV) in esophageal cancer relies on computed tomography (CT) and combination with 18F-fluorodeoxyglucose (FDG) positron emission tomography (PET). There is increasing interest in integrating magnetic resonance imaging (MRI) in radiation treatment, which can potentially obviate CT- or FDG-PET/CT based delineation. The aim of this study is to evaluate the feasibility of target delineation on T2-weighted (T2W) MRI and T2W including diffusion-weighted MRI (T2W+DW-MRI) compared with current-practice FDG-PET/CT.
\end{abstract}

Methods

Ten observers delineated primary esophageal tumor GTVs of 6 patients on FDG-PET/CT, T2WMRI, and T2W+DW-MRI. GTVs, generalized conformity indices, in-slice delineation variation (root mean square), and standard deviations in the position of the most cranial and caudal delineated slice were calculated.

Results

Delineations on MRI showed smaller GTVs compared with FDG-PET/CT-based delineations. The main variation was seen at the cranial and caudal border. No differences were observed in conformity indices (FDG-PET/CT, 0.68; T2W-MRI, 0.66; T2W+ DW-MRI, 0.68) and in-slice variation (root mean square, $0.13 \mathrm{~cm}$ on FDG-PET/CT; $0.10 \mathrm{~cm}$ on T2W-MRI; $0.14 \mathrm{~cm}$ on T2W+DW-MRI). In the 2 tumors involving the gastroesophageal junction, addition of DW-MRI to T2W-MRI significantly decreased caudal border variation.

\title{
Conclusions
}

MRI-based target delineation of the esophageal tumor is feasible with interobserver variability comparable to that with FDG-PET/CT, despite limited experience with delineation on MRI. Most variation was seen at cranial-caudal borders, and addition of DW-MRI to T2W-MRI may reduce caudal delineation variation of gastroesophageal junction tumors. 


\section{INTRODUCTION}

Esophageal cancer is diagnosed in $>450,000$ patients per year worldwide and is the sixth most common cause of cancer-related death'. Standard therapy with curative intent for patients with locally advanced esophageal cancer consists of neoadjuvant chemoradiation therapy followed by surgery, improving 5-year survival compared with surgery alone ${ }^{2,3}$. For patients who are unfit for major surgery, definitive chemoradiation therapy is preferred ${ }^{4}$. Thus, radiation treatment plays a central role in the treatment of esophageal cancer ${ }^{5}$. Accurate gross tumor volume (GTV) delineation of the primary tumor is essential when boost strategies are applied. Increasing evidence suggests that boosting gross primary disease may improve local tumor control ${ }^{6}$. Implementation of simultaneous integrated boost techniques may offer the advantage of delivering a higher dose to the tumor while maintaining conventional doses to subclinical disease ${ }^{7}$.

Currently, delineation of esophageal tumors is performed on computed tomography (CT), and the added value of 18F-fluorodeoxyglucose (FDG) positron emission tomography (PET) has been explored $^{8-10}$. Even with FDG-PET/CT fusion, GTV interobserver variability remains high in some cases, especially at the cranial and caudal tumor borders ${ }^{11}$.

On-board online cone beam CT imaging contributed to the development of image guided radiation therapy, which improved precision of radiation therapy setup in esophageal cancer ${ }^{12}$. However, cone beam CT offers suboptimal soft-tissue contrast, and imaging of moving organs can be difficult. Because of its superior differentiation of soft tissues, integration of magnetic resonance imaging (MRI) in radiation therapy is promising ${ }^{13,14}$. The clinical implementation of MRI before and during each fraction of radiation therapy is being explored with the introduction of the Unity MRI-linear accelerator (MR-Linac; Elekta AB, Stockholm, Sweden). The MR-Linac combines 1.5 T MRI with a state-of-the-art linear accelerator and an online adaptive workflow ${ }^{15,16}$. Continuous adaptation of treatment based on daily MRI scans has the potential to improve radiation precision.

MRI of the esophagus has been improved over the past years. Artefacts in esophageal MRI scans from movement of the esophagus have been reduced by technique optimizations, resulting in highquality MRI. For instance, positioning a navigator on the diaphragm allows for image acquisition in expiration only, thereby reducing motion artefacts ${ }^{17,18}$. On MRI the individual layers of the esophageal wall can be clearly visualized ${ }^{19,20}$ and a good correlation of T-stage on MRI with histopathologic T-stage has been described ${ }^{21}$. For anatomic visualization and staging of esophageal tumors, MRI may even be superior to other imaging strategies ${ }^{22}$. MRI also provides the opportunity to perform functional imaging, such as diffusion-weighted (DW) MRI, a valuable cancer imaging biomarker measuring tumor physiology ${ }^{23}$. DW-MRI depends on the reduction in diffusion within the water microenvironment ${ }^{24,25}$. An increase in cell density, secondary to fast cell proliferation in tumors, results in a high signal on DW images. A previous study showed that longitudinal tumor lengths of esophageal cancers are more accurately delineated using DW-MRI compared with CT or T2-weighted (T2W) MRI only26. 
Delineation studies in other tumor subtypes (eg, head and neck, prostate, and pancreatic cancer) already showed promising results regarding delineation of the GTV on MRI ${ }^{27-30}$. However, delineation variation of the GTV in esophageal cancer on MRI compared with FDG-PET/CT is unknown.

Because of the increasing interest in integrating MRI into radiation treatment, the aim of this study is to evaluate target delineation on T2W-MRI and T2W+DW-MRI compared with current-practice FDG-PET/CT.

\section{MATERIALS AND METHODS}

\section{Patients}

Six patients, diagnosed with locally advanced esophageal cancer between December 2013 and December 2014, were prospectively included in a study evaluating response to neoadjuvant chemoradiation therapy of esophageal tumors by means of MRI and FDG-PET/CT examinations. This study was approved by the local medical ethics committee, and written informed consent was obtained from all patients (NCT 02125448). The present study concerns an ancillary study evaluating the feasibility of delineation on MRI compared with FDG-PET/CT before the start of neoadjuvant chemoradiation. Four patients were male, and 2 were female, with a mean age of 67 years (range, 54-74 years). We included squamous cell carcinomas $(n=3)$ and adenocarcinomas $(n=3)$. The patient characteristics are displayed in Table 1. All patients underwent a FDG-PET/ CT scan and endoscopy combined with endoscopic ultrasound (EUS) as standard of care. An additional MRI scan was acquired before treatment. After image acquisition, all patients were treated with chemoradiation therapy followed by esophagectomy.

Table 1. Baseline characteristics of the 6 esophageal cancer cases

\begin{tabular}{|c|c|c|c|c|c|}
\hline Case & cTNM* & AJCC locationt & Histology & Male/Female & Age (yr) \\
\hline 1 & T3N2MO & Upper thoracic & SCC & $M$ & 67 \\
\hline 2 & T3N1-2MO & Middle thoracic & SCC & $M$ & 54 \\
\hline 3 & $\mathrm{~T} 2 \mathrm{~N} 2 \mathrm{MO}$ & Lower thoracic, GEJキ & $A C$ & $M$ & 71 \\
\hline 4 & T3N2-3MO & Lower thoracic, GEJ & $A C$ & $\mathrm{~F}$ & 70 \\
\hline 5 & T2No-1MO & Lower thoracic & $A C$ & $M$ & 64 \\
\hline 6 & T3N1MO & Upper thoracic & SCC & $\mathrm{F}$ & 74 \\
\hline \multicolumn{6}{|c|}{$\begin{array}{l}\text { Abbreviations: } \mathrm{AC}=\text { adenocarcinoma; } \mathrm{GEJ}=\text { gastroesophageal junction; } \mathrm{SCC}=\text { squamous cell carcinoma. } \\
\text { * Clinical tumor-node-metastasis (cTNM) stage } \mathrm{e}^{42} \text {. } \\
\text { † American Joint Committee on Cancer classification } 2012^{42} \text {. } \\
\text { † Lower thoracic fumor involving the GEJ. }\end{array}$} \\
\hline
\end{tabular}




\section{Image acquisition}

\section{FDG-PET/CT}

The FDG-PET/CT scan was performed in radiation therapy treatment position on an integrated hybrid system combining multidetector CT and FDG-PET (Siemens, Erlangen, Germany). All patients were required to fast for at least 6 hours before the injection of 18F-FDG. Blood glucose levels were checked in every patient to exclude hyperglycemia. 18F-FDG (2.0 mega- Becquerels/kg) was intravenously injected 60 minutes before scanning. A CT scan was performed for attenuation correction purposes, and the PET was acquired 3 dimesionally with a scan time of 3 minutes per bed position. 18F-FDG-PET/CT reconstruction was performed with ordered-subsets expectation maximization for 21 subsets and 4 iterations (Gaussian filter).

MRI

MRI scans were performed on a 1.5 T MRI scanner (Philips Achieva or Ingenia, Best, the Netherlands) using TorsoXL (16 channels) or Anterior/Posterior (28 channel) receiver coils. MRI scanning consisted of T2W-MRI and DW-MRI in axial planes with a slice thickness of $6.5 \mathrm{~mm}$. A respiratory navigator was positioned on the diaphragm to reduce motion artefacts, and scans were only acquired in expiration ${ }^{18}$. The $b=800 \mathrm{~s} / \mathrm{mm}^{2}$ images were used for delineation of the tumor. Apparent diffusion coefficient (ADC) maps are quantitative measurements of tissue diffusivity, calculated over different $b$-values, and can also be visually displayed ${ }^{24,25}$. These visual displayed $A D C$ maps, calculated from $b=0, b=200$, and $b=800 \mathrm{~s} / \mathrm{mm}^{2}$, were available during delineation.

\section{Observers}

The GTV of the primary tumor of the 6 cases was independently delineated by 10 observers from 2 centers in the Netherlands. First, they delineated the GTV on fused FDG-PET/CT only. After a minimum interval of 2 weeks, delineations of the GTV were repeated on T2W-MRI only. Thereafter, DW-MRI of $b=800 \mathrm{~s} / \mathrm{mm}^{2}$ and ADC maps were added and delineations were adjusted to create a GTV based on a combination of T2W and DW-MRI.

\section{Delineation guidelines}

All clinical information of the cases (age, sex, histology, endoscopy report, EUS report, and FDG$\mathrm{PET} / \mathrm{CT}$ report) was provided, reflecting clinical practice. Observers delineated target volumes using Volumetool, a software tool developed at the University Medical Center Utrecht, the Netherlands. Before the start, 2 consensus meetings were organized to discuss magnetic resonance delineation guidelines for esophageal cancer based on the available literature. Gastrointestinal expert radiation oncologists, radiologists, and researchers in the field of esophageal cancer imaging from the 2 centers participated in these meetings. A digital manual was sent to the observers with 
the consensus guidelines for delineation on MRI. The observers were instructed to delineate the primary tumor GTV and to exclude potential suspicious locoregional lymph nodes on all delineation modalities. For delineation on MRI, observers were provided with the FDG-PET/CT report but were not allowed to review the noncoregistered PET/CT on a second screen.

\section{FDG-PET/CT delineations}

The mean activity in the liver served as the reference for physiologic uptake of FDG in a fasting patient ${ }^{31}$, and a nuclear medicine physician standardized the windowlevel for all cases before FDG-PET/CT delineation. Observers were instructed to delineate the tumor on CT and adjust delineations after coregistration with FDG-PET. Observers were informed about the slice thickness of $3 \mathrm{~mm}$.

T2W-MRI and T2W+DW-MRI delineations

An identical window level and grayscale per patient was preset for the T2W-MRI delineations. Observers were informed about the slice thickness of $6.5 \mathrm{~mm}$. After addition of DW-MRI using $b$ $=800 \mathrm{~s} / \mathrm{mm}^{2}$ images, delineations were adjusted on T2W+DW-MRI. Observers received visually displayed $A D C$ maps (calculated from $b=0, b=200$, and $b=800 \mathrm{~s} / \mathrm{mm}^{2}$ ) to determine possible T2 shine-through effects, which result in a high signal intensity in the $b=800 \mathrm{~s} / \mathrm{mm}^{2}$ image but are not related to true diffusion restriction.

Volumetric analysis

The mean and standard deviations (SDs) of the delineated GTVs were calculated per patient and compared between FDG-PET/CT, T2W-MRI, and T2W+DW-MRI delineations.

\section{Contour analysis}

Overlap analysis

To quantify the overlap among FDG-PET/CT-, T2W-MRI-, and T2W+DW-MRI-based delineations, we calculated the generalized conformity index (Clgen), defined as the sum of the common volumes between observer pairs divided by the sum of the encompassing volumes between each pair of observers. A Clgen of 1 indicates 100\% agreement between observers, and a Clgen of 0 indicates no overlap in delineation. Clgens were calculated per patient and averaged over all patients per modality ${ }^{32}$.

Delineation variation of the central region: in-slice SD

The central delineated region was defined as the region that was delineated by all 10 observers. For this region, a surface distance variation was calculated. A reference contour for each patient was computed in 3 dimensions and denoted the median surface GTV ${ }^{33}$. This median surface, 
encircling 50\% coverage of the GTVs of all observers, was sampled using 8000 equally distributed points. For all points, the perpendicular distance to each delineated GTV surface was calculated. The variation of the different observers for each point on the median surface was expressed in a local observer variation (local SD). For the central delineated region, the overall observer variation was calculated for every patient as the quadratic mean of the local SD, demonstrating in slice SD.

Cranial and caudal tumor border variation

The cranial and caudal variation of the esophageal tumor delineations was calculated as the SD of the most proximal and distal delineated slice. As a result of the difference in slice thickness between FDG-PET/CT (3 mm) and T2W+DW-MRI $(6.5 \mathrm{~mm})$, SDs of delineations in the most cranial and caudal delineated slice could only be compared between T2W-MRI and T2W+DW-MRI ${ }^{34}$.

Statistical methods

GTVs and generalized conformity indices were compared using a pairwise t test (paired 2-sided Student $t$ test). To compare SDs, the 2-sided F-test was used ${ }^{34}$. P values lower than .05 were considered statistically significant.

\section{RESULTS}

Volumetric analysis

The 10 observers delineated significantly smaller absolute GTVs on T2W-MRI and T2W+DW-MRI compared with FDG-PET/CT ( $P=.07$ for T2W-MRI vs FDG-PET/CT and $P=.01$ for T2W+DW-MRI vs FDG-PET/CT; Table 2). The FDG-PET/CT-based mean GTVs over all patients was $40.5 \mathrm{~cm}^{3}$ (SDper-patient range, 0.8-9.7 $\mathrm{cm}^{3}$ ). The T2W-MRI-based mean GTV was $34.8 \mathrm{~cm}^{3}$ (SD-per-patient range, $1.4-16.1 \mathrm{~cm}^{3}$ ). On T2W+DW-MRI, the mean GTV decreased to $32.7 \mathrm{~cm}^{3}$ (SD-per-patient range, $1.7-8.2 \mathrm{~cm}^{3}$ ).

Overlap analysis

The mean generalized Clgen over all patients was 0.68 on FDG-PET/CT, 0.66 on T2W-MRI, and 0.68 on T2W+DW-MRI (Table 2), which was not significantly different across modalities $(P=.68$ for FDG-PET/CT vs T2W-MRI; P = .83 for FDG-PET/CT vs T2W+DW-MRI; and P = .26 for T2WMRI vs T2W+DW-MRI).

Delineation variation of the central region: in-slice SD

The in-slice SD of the central delineated region was small in the 6 included cases and did not differ between modalities (mean, $0.13 \mathrm{~cm}$ on FDG-PET/CT, $0.10 \mathrm{~cm}$ on T2W-MRI, and $0.14 \mathrm{~cm}$ on T2W+DW-MRI; Table 2). 
Table 2. Volumetric index and interobserver variation of FDG-PET/CT-, T2W-MRI-, and T2W+DWMRI -based GTV delineations

\begin{tabular}{|c|c|c|c|c|c|c|c|c|c|}
\hline \multirow[b]{2}{*}{ Case } & \multicolumn{3}{|c|}{ Mean volume $\left(\mathrm{cm}^{3}\right)^{*}$} & \multicolumn{3}{|c|}{$\begin{array}{c}\text { Generalized conformity } \\
\text { index (Clgen) }\end{array}$} & \multicolumn{3}{|c|}{$\begin{array}{l}\text { Central overall SD } \\
(\mathrm{RMS}, \mathrm{cm}) \dagger\end{array}$} \\
\hline & $\mathrm{PET} / \mathrm{CT}$ & T2W-MRI & $\begin{array}{c}\text { T2W+ } \\
\text { DW-MRI }\end{array}$ & $\begin{array}{l}\text { PET/ } \\
\text { CT }\end{array}$ & $\begin{array}{l}\text { T2W- } \\
\text { MRI }\end{array}$ & $\begin{array}{c}\text { T2W+ } \\
\text { DW-MRI }\end{array}$ & PET/CT & T2W-MRI & $\begin{array}{c}\text { T2W+ } \\
\text { DW-MRI }\end{array}$ \\
\hline 1 & $47.8 \pm 7.0$ & $40.6 \pm 6.3$ & $38.2 \pm 6.1$ & 0.68 & 0.68 & 0.72 & 0.13 & 0.06 & 0.07 \\
\hline 2 & $46.3 \pm 3.7$ & $32.7 \pm 4.2$ & $32.4 \pm 4.5$ & 0.75 & 0.75 & 0.79 & 0.13 & 0.09 & 0.09 \\
\hline 3 & $75.5 \pm 9.7$ & $63.8 \pm 8.9$ & $64.6 \pm 6.1$ & 0.74 & 0.74 & 0.67 & 0.11 & 0.14 & 0.14 \\
\hline 4 & $39.6 \pm 8.1$ & $38.0 \pm 16.1$ & $33.1 \pm 8.2$ & 0.62 & 0.62 & 0.43 & 0.20 & 0.12 & 0.27 \\
\hline 5 & $22.5 \pm 8.6$ & $21.9 \pm 3.8$ & $16.4 \pm 3.6$ & 0.53 & 0.53 & 0.62 & 0.08 & 0.08 & 0.10 \\
\hline 6 & $11.2 \pm 0.8$ & $11.7 \pm 1.4$ & $11.5 \pm 1.7$ & 0.73 & 0.73 & 0.71 & 0.09 & 0.10 & 0.09 \\
\hline \multirow[t]{2}{*}{ Mean } & 40.5 & 34.8 & 32.7 & 0.68 & 0.68 & 0.68 & 0.13 & 0.10 & 0.14 \\
\hline & $p=$ & $p=0.01$ & 1 & & & & & & \\
\hline
\end{tabular}

Abbreviations; FDG-PET/CT = fluorodeoxyglucose positron emission tomography computed tomography; T2W = T2weighted; DW = diffusion-weighted; $M R I=$ magnetic resonance imaging; $G T V=$ gross tumour volume; $S D=$ standard deviation; RMS = Root Mean Square, $\mathrm{cm}=$ centimetre

*Mean per patient \pm standard deviation. $\mathrm{p}<0.05$ considered statistically significant, calculated between the means per modality, paired-sample T-test (2-tailed).

†Central overall SD = quadratic mean of the local SD weighted for surface (RMS) of the central part of the median surface where $100 \%$ of the observers agreed delineations.

Cranial and caudal tumor border variation

On all modalities, delineation variability mainly occurred at the cranial and caudal tumor borders. SDs in the position of the most cranial and caudal delineated slice are displayed in Table 3, with significance levels calculated to report delineation differences between T2W-MRI and T2W+DWMRI. Figure 1 visualizes slice variation at the proximal and distal border for delineations on FDGPET/CT, T2W-MRI, and T2W+DW-MRI. Case 1 showed large delineation variation at the cranial border. In this case, satellite lesions were present cranially of the tumor, which were described in the endoscopic report. These lesions were included in the GTV by 4 of 10 physicians on FDG-PET/ CT, T2W-MRI, and DW-MRI. In case 3 and 4 with gastroesophageal junction (GEJ) involvement, a significant decrease in caudal border variation was observed on T2W+DW-MRI compared with T2W-MRI (P = .04 in case 3, P = .01 in case 4; Table 3). Figure 2 shows an example of GTV delineation variation at the GEJ on T2W-MRI (Fig 2a) and T2W+DW-MRI (Fig 2c), displaying improved delineation agreement at the caudal border after addition of DW-MRI. 
Table 3. Delineation variation on FDG-PET/CT, T2W-MRI, and T2W+DW-MRI at the cranial and caudal tumor borders

\begin{tabular}{|c|c|c|c|c|c|c|c|c|}
\hline \multirow[b]{2}{*}{ Case } & \multicolumn{4}{|c|}{ Cranial delineation variation $1 \mathrm{SD}(\mathrm{cm}) \dagger$} & \multicolumn{4}{|c|}{ Caudal delineation variation $1 S D(\mathrm{~cm}) \dagger$} \\
\hline & $\mathrm{PET} / \mathrm{CT}$ & T2W-MRI & $\begin{array}{c}\text { T2W+DW- } \\
\text { MRI }\end{array}$ & $p^{*}$ & $\mathrm{PET} / \mathrm{CT}$ & T2W-MRI & & $p^{*}$ \\
\hline 1 & 1.25 & 1.38 & 1.30 & 0.86 & 0.39 & 0.57 & 0.53 & 0.84 \\
\hline 2 & 0.35 & 0.21 & 0.21 & 1.00 & 0.20 & 0.37 & 0.33 & 0.78 \\
\hline 3 & 0.09 & 0.55 & 0.37 & 0.25 & 0.84 & 0.76 & 0.37 & 0.04 \\
\hline 4 & 0.67 & 1.22 & 1.00 & 0.56 & 0.21 & 1.22 & 0.46 & 0.01 \\
\hline 5 & 1.22 & 0.44 & 0.33 & 0.44 & 0.57 & 0.94 & 0.82 & 0.69 \\
\hline 6 & 0.20 & 0.27 & 0.31 & 0.69 & 0.17 & 0.45 & 0.53 & 0.65 \\
\hline
\end{tabular}

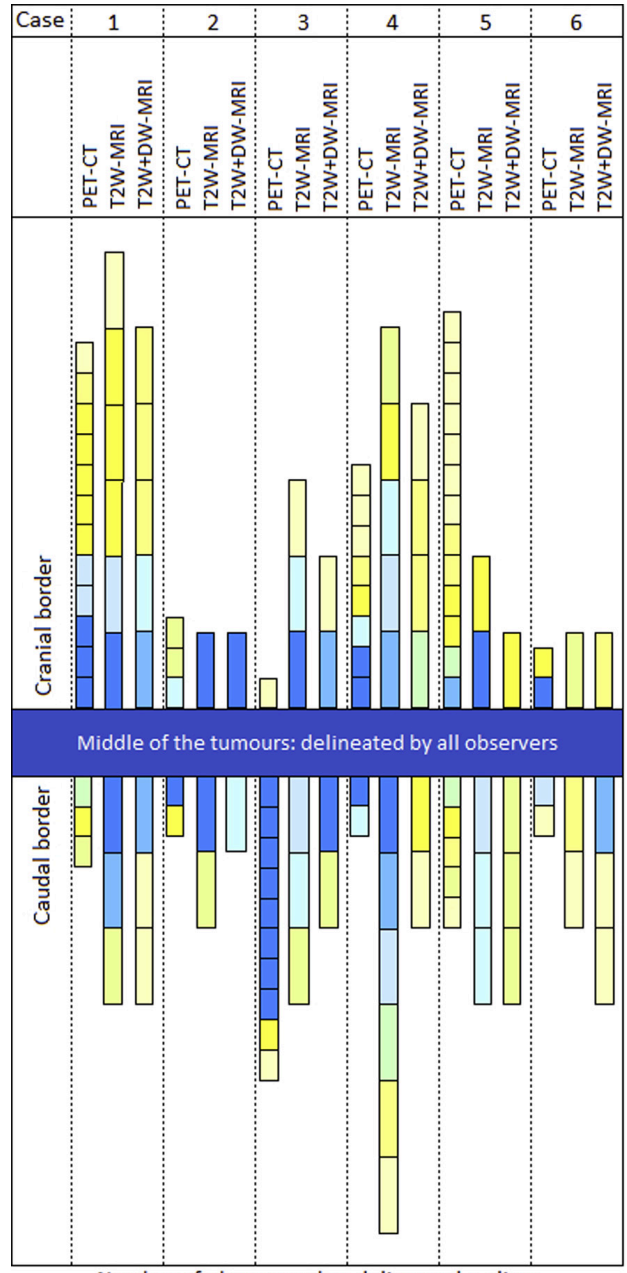

Number of observers that delineated a slice: \begin{tabular}{|l|l|l|l|l|l|l|l|l|l|}
\hline 10 & 9 & 8 & 7 & 6 & 5 & 4 & 3 & 2 & 1 \\
\hline
\end{tabular}
Figure 1. Slice variation on FDG-PET/CT, T2W-MRI, and T2W+DW-MRI at the cranial and caudal tumor borders.

Each block represents a delineated slice. The number of observers delineating a slice is displayed under the figure. The length of the blocks varies according to the slice thickness (which was $3 \mathrm{~mm}$ on FDG-PET/CT and 6.5 $\mathrm{mm}$ on MRI).

Abbreviations: FDG-PET/CT =

fluorodeoxyglucose positron emission tomography computed tomography; $\mathrm{T} 2 \mathrm{~W}=\mathrm{T} 2$-weighted; $\mathrm{DW}=$ diffusionweighted; $M R I=$ magnetic resonance imaging. 


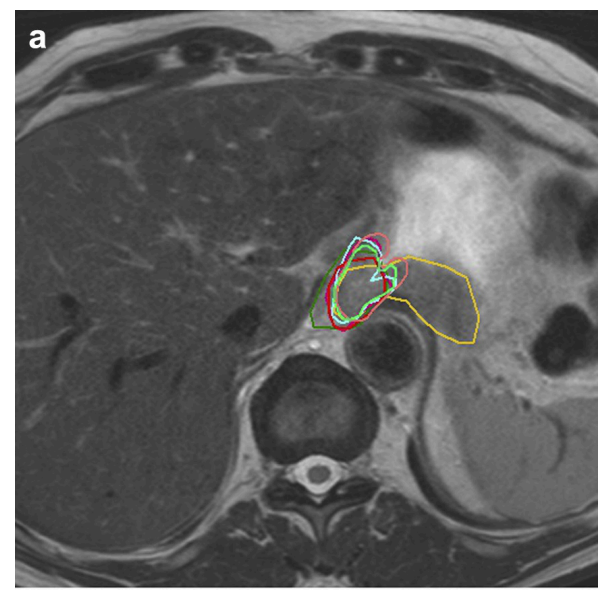

\section{b}

Figure 2. A 71-year-old male patient (case 3) with a tumor involving the gastroesophageal junction.

a. Delineations of the esophageal GTV of the 10 observers on T2-weighted MRI only.

b. The added diffusion-weighted MRI scan of the same slice, showing the differentiation between tumor and gastric wall thickening.

c. Delineations adjusted after the addition of diffusion-weighted MRI.

Abbreviations: GTV = gross tumor volume; $M R I=$ magnetic resonance imaging . 


\section{DISCUSSION}

This multiobserver study shows that delineation of the primary esophageal tumor on MRI is feasible, with interobserver variability for GTV delineation on MRI comparable to that with FDG-PET/CT. The main variation is seen at the cranial and caudal tumor border, whereas centrally, differences in tumor delineations were small on T2W and T2W+DW-MRI. The addition of DW-MRI to T2WMRI in the 2 GEJ-involving tumors significantly reduced the SD of the most caudal delineated slice, showing the potential value of DW-MRI for delineation of the caudal border in GEJ-involving cases. To our knowledge, this is the first study comparing GTV delineation variability on T2W-MRI and T2W+DW-MRI to FDG-PET/CT for esophageal cancer. Currently, the gold standard for delineation of esophageal cancer is CT (with fused PET if available) and correlation with endoscopy/EUS findings. Delineation on $\mathrm{CT}$ only is challenging, mainly in differentiating tumor from normal tissue at the cranial and caudal tumor borders. Delineation on FDG-PET/CT may help in determining these tumor borders; however, FDG uptake does not differentiate between tumor and inflammation, which can be difficult especially in tumors involving the GE ${ }^{35,36}$. Furthermore, on FDG-PET/CT the target volume can depend on the threshold chosen and may increase or decrease depending on the windowing. On the contrary, this study showed promising results for delineation on MRI in patients with esophageal cancer. Especially for cases with GEJ involvement, the addition of DW imaging to T2W-MRI showed additional value for T2W-MRI only.

In this study, GTVs were significantly smaller on MRI compared with FDG-PET/CT. This may partly be caused by the acquisition of MRI at the end of the expiration, whereas for FDG-PET/CT no motion-specific scanning techniques were used, showing the extent of malignancy throughout the breathing cycle. Furthermore, the finding of a reduced GTV on esophageal MRI is in line with a study by Hou et al ${ }^{26}$. They compared longitudinal length accuracy of esophageal cancer cases on T2W-MRI and DW-MRI to CT and found that DW-MRI resulted in the smallest fumor lengths. Another study showed a good correlation between DW-MRI tumor lengths and histopathologic tumor length ${ }^{26}$. In prostate and cervical cancer, delineations on DW-MRI resulted in slightly smaller GTVs compared with histopathology $3^{37,38}$. An important matter is that smaller GTVs may translate into smaller clinical target volumes when automatic expansion is used, which theoretically could lead to underdosage of microscopic disease. The optimal approach to investigate the accuracy of delineation on MRI would be a direct correlation between a preoperative MRI in patients who are planned to undergo direct esophagectomy and histopathology. Because patients with locally advanced esophageal cancer are treated with neoadjuvant therapy (chemoradiation therapy or chemotherapy) before surgery, direct correlation of imaging to pathology cannot be performed. An alternative approach is to investigate local control rates, focusing on potential rim recurrences, when delineation on MRI is integrated in a magnetic resonance-based workflow.

A limitation of this study is the mean interval of 14 days between FDG-PET/CT and MRI acquisition, which limits the comparability between the different modalities in terms of tumor volume in light of 
potential tumor growth. Therefore, the main focus was the comparison of interobserver variability between modalities (demonstrated as Clgens and craniocaudal delineation variation). Second, MRI scans were acquired in the axial plane only (with sagittal reconstructions available), and addition of sagittal plane imaging of the tumors might further optimize delineation accuracy, especially in determining the cranial and caudal tumor extension. Third, the slice thickness of the MRI scans was $6.5 \mathrm{~mm}$, and reducing this slice thickness may lead to further improvement in the accuracy in GTV delineations. Lastly, the number of patients in this study was limited to 6 . Future studies should include a larger number of patients to validate our results.

The importance of delineation guidelines in radiation therapy is well known and has been studied for CT and FDG-PET/CT in different tumor sites $34,39,40$. For delineation on MRI, the importance of guidelines has been shown for head and neck, pancreatic, and prostate cancer studies ${ }^{27-30}$. Consensus guidelines for esophageal cancer have been developed for CT and FDG-PET/CT contouring $^{41}$. Although GTV delineation was comparable on MRI and FDG-PET/CT, this study emphasizes the need to expand clinical experience on magnetic resonance delineation for esophageal cancer and to implement robust delineation guidelines. GTVs on MRI were overall smaller compared with those on FDG-PET/CT, and the impact on clinical practice is unknown. To ensure accurate GTV delineation of the macroscopic tumor and mirror clinical practice, we recommend further delineation studies on anatomic T2W-MRI combined with DW-MRI.

\section{Conclusions}

MRI-based delineation of the esophageal GTV shows target delineation variability comparable to that with FDG-PET/CT, although overall GTVs were smaller on MRI compared with FDG-PET/ CT. The addition of DW-MRI to T2W-MRI potentially facilitates delineation of the caudal border in GEJ tumors. Future research should focus on refinement of esophageal MRI acquisition and the development of internationally validated delineation guidelines to implement MRI delineation in esophageal radiation therapy clinics. 


\section{REFERENCES}

1. FerlayJ, Soerjomataram I, DikshitR, etal. Cancer incidence and mortality worldwide: sources, methods and major patterns in GLOBOCAN 2012. Int J Cancer 2015;136:E359-86.

2. van Hagen $P$, Hulshof $M C$, van Lanschot JJ, et al. Preoperative chemoradiotherapy for esophageal or junctional cancer. N Engl J Med 2012;366:2074-84.

3. Shapiro J, van Lanschot JJB, Hulshof MCCM, et al. Neoadjuvant chemoradiotherapy plus surgery versus surgery alone for oesophageal or junctional cancer (CROSS): long-term results of a randomised controlled trial. Lancet Oncol 2015;16:1090-8.

4. Bedenne L, Michel P, Bouche $\mathrm{O}$, et al. Chemoradiation followed by surgery compared with chemoradiation alone in squamous cancer of the esophagus: FFCD 9102. J Clin Oncol 2007;25:1160-8.

5. Lordick F, Mariette C, Haustermans K, Obermannova R, Arnold D, Committee EG. Oesophageal cancer: ESMO Clinical Practice Guidelines for diagnosis, treatment and followup. Ann Oncol 2016;27:v50-v7.

6. Welsh JW, Seyedin SN, Allen PK, et al. Local Control and Toxicity of a Simultaneous Integrated Boost for Dose Escalation in Locally Advanced Esophageal Cancer: Interim Results from a Prospective Phase I/II Trial. J Thorac Oncol 2017; 12:375-82.

7. Deng $\mathrm{W}$, Lin SH. Advances in radiotherapy for esophageal cancer. Ann Transl Med 2018;6:79.

8. Vesprini $D$, Ung $Y$, Dinniwell $R$, et al. Improving observer variability in target delineation for gastro-oesophageal cancer--the role of (18F) fluoro-2-deoxy-D-glucose positron emission tomography/computed tomography. Clin Oncol (R Coll Radiol) 2008;20:631-8.

9. Muijs CT, Schreurs LM, Busz DM, et al. Consequences of additional use of PET information for target volume delineation and radiotherapy dose distribution for esophageal cancer. Radiother Oncol 2009;93:447-53.

10. Muijs CT, Beukema JC, Woutersen D, et al. Clinical validation of FDG-PET/CT in the radiation treatment planning for patients with oesophageal cancer. Radiother Oncol 2014; 113:188-92.

11. Schreurs LM, Busz DM, Paardekooper GM, et al. Impact of 18-fluorodeoxyglucose positron emission tomography on computed tomography defined target volumes in radiation treatment planning of esophageal cancer: reduction in geographic misses with equal inter-observer variability: PET/CT improves esophageal target definition. Dis Esophagus 2010;23:493-501.

12. van Nunen $A$, van der Sangen $M J C$, van Boxtel M, van Haaren PMA. Cone-Beam CTbased position verification for oesophageal cancer: Evaluation of registration methods and anatomical changes during radiotherapy. Tech Innov Patient Support Radiat Oncol 2017;3$4: 30-6$.

13. Menard C, van der Heide U. Introduction: Systems for magnetic resonance image guided radiation therapy. Semin Radiat Oncol 2014;24:192.

14. Pollard JM, Wen Z, Sadagopan R, Wang $J$, Ibbott GS. The future of image-guided radiotherapy will be MR guided. Br J Radiol 2017;90:20160667.

15. Kerkmeijer LG, Fuller CD, Verkooijen HM, et al. The MRI-Linear Accelerator Consortium: Evidence-Based Clinical Introduction of an Innovation in Radiation Oncology Connecting Researchers, Methodology, Data Collection, Quality Assurance, and Technical Development. Front Oncol 2016;6:215.

16. Schultz C. MR-linac: Clinical introduction. Radiother Oncol 2016;119:S232.

17. Ehman RL, Felmlee JP. Adaptive technique for high-definition MR imaging of moving structures. Radiology 1989;173:255-63.

18. Lever FM, Lips IM, Crijns SP, et al. Quantification of esophageal tumor motion on cine-magnetic resonance imaging. Int J Radiat Oncol Biol Phys 2014;88:419-24.

19. Riddell AM, Davies DC, Allum WH, Wotherspoon AC, Richardson C, Brown G. High-resolution $M R I$ in evaluation of the surgical anatomy of the esophagus and posterior mediastinum. AJR Am J Roentgenol 2007;188:W37-43. 
20. Riddell AM, Richardson C, Scurr E, Brown G. The development and optimization of high spatial resolution MRI for imaging the oesophagus using an external surface coil. $\mathrm{Br} \mathrm{J}$ Radiol 2006;79:873-9.

21. Yamada I, Miyasaka N, Hikishima K, et al Ultra-high-resolution MR imaging of esophageal carcinoma at ultra-high field strength (7.0T) ex vivo: correlation with histopathologic findings. Magn Reson Imaging 2015;33:413-9.

22. van Rossum PS, van Hillegersberg R, Lever FM, et al. Imaging strategies in the management of oesophageal cancer: what's the role of MRI? Eur Radiol 2013;23:1753-65.

23. Padhani AR, Liu G, Mu-Koh D, et al. DiffusionWeighted Magnetic Resonance Imaging as a Cancer Biomarker: Consensus and Recommendations. Neoplasia 2009;11:10225 .

24. Le Bihan D, Poupon C, Amadon A, Lethimonnier F. Artifacts and pitfalls in diffusion MRI. J Magn Reson Imaging 2006;24:478-88.

25. KohDM, Collins DJ. Diffusion-weighted MRI in the body: applications and challenges in oncology. AJR Am J Roentgenol 2007;188:1622-35.

26. Hou DL, Shi GF, Gao XS, et al. Improved longitudinal length accuracy of gross tumor volume delineation with diffusion weighted magnetic resonance imaging for esophageal squamous cell carcinoma. Radiat Oncol 2013;8:169.

27. Rud E, Klotz D, Rennesund K, et al. Detection of the index tumour and tumour volume in prostate cancer using T2-weighted and diffusionweighted magnetic resonance imaging (MRI) alone. BJU Int 2014;1 14:E32-E42.

28. Dinh CV, Steenbergen P, Ghobadi G, et al. Magnetic resonance imaging for prostate cancer radiotherapy. Phys Med 2016;32:446-51.

29. Jager EA, Ligtenberg H, Caldas-Magalhaes J, et al. Validated guidelines for tumor delineation on magnetic resonance imaging for laryngeal and hypopharyngeal cancer. Acta Oncol 2016;55:1305-12.

30. Heerkens HD, Hall WA, Li XA, et al. Recommendations for MRI-based contouring of gross tumor volume and organs at risk for radiation therapy of pancreatic cancer. Pract
Radiat Oncol 2017;7:126-36.

31. Chirindel A, Alluri KC, Tahari AK, et al. Liver standardized uptake value corrected for lean body mass at FDG PET/CT: effect of FDG uptake time. Clin Nucl Med 2015;40:e17-22.

32. Kouwenhoven E, Giezen M, Struikmans H. Measuring the similarity of target volume delineations independent of the number of observers. Phys Med Biol 2009;54:2863-73.

33. Deurloo KE, Steenbakkers RJ, Ziip U, et al. Quantification of shape variation of prostate and seminal vesicles during external beam radiotherapy. Int J Radiat Oncol Biol Phys 2005;61:228-38

34. Niikamp J, de Haas-Kock DF, Beukema JC, et al. Target volume delineation variation in radiotherapy for early stage rectal cancer in the Netherlands. Radiother Oncol 2012;102:1421.

35. Horton KM, Fishman EK. Current Role of CT in Imaging of the Stomach. RadioGraphics 2003;23:75-87.

36. Kobayashi S, Ogura $M$, Suzawa $N$, et al. 18F-FDG uptake in the stomach on screening PET/CT: value for predicting Helicobacter pylori infection and chronic atrophic gastritis. BMC Medical Imaging 2016;16:58

37. de Boer P, Bleeker MC, Spijkerboer AM, et al. Craniocaudal tumour extension in uterine cervical cancer on MRI compared to histopathology. Eur J Radiol Open 2015;2:11 17.

38. Le Nobin J, Orczyk C, Deng FM, et al. Prostate tumour volumes: evaluation of the agreement between magnetic resonance imaging and histology using novel co-registration software. BJU Int 2014;114:E105-E12.

39. Lim K, Small W, Portelance L, et al. Consensus Guidelines for Delineation of Clinical Target Volume for Intensity-Modulated Pelvic Radiotherapy for the Definitive Treatment of Cervix Cancer. Int J Radiat Oncol Biol Phys $2011 ; 79: 348-55$.

40. Michalski JM, Lawton C, El Naqa I, et al. Development of RTOG Consensus Guidelines for the Definition of the Clinical Target Volume for Postoperative Conformal Radiation Therapy for Prostate Cancer. Int J Radiat Oncol Biol Phys 
$2010 ; 76: 361-8$

41. Wu AJ, Bosch WR, Chang DT, et al. Expert Consensus Contouring Guidelines for Intensity Modulated Radiation Therapy in Esophageal and Gastroesophageal Junction Cancer. Int J Radiat Oncol Biol Phys 2015;92:911-20.

42. Rice TW, Blackstone EH, Rusch VW. 7th edition of the AJCC Cancer Staging Manual: esophagus and esophagogastric junction. Ann Surg Oncol 2010;17:1721-4. 


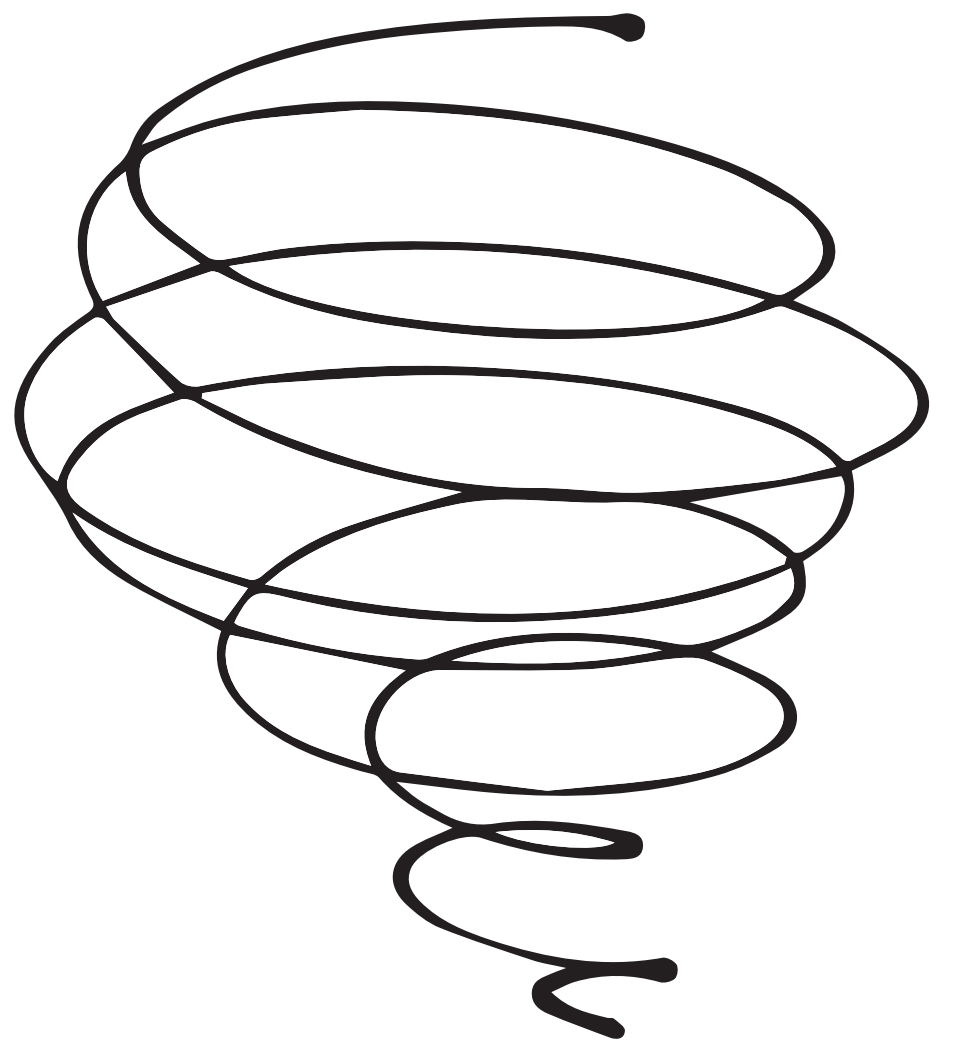




\section{CHAPTER 7}

\section{General Discussion}


This thesis aims to investigate the role of DW-MRI in response assessment after neoadjuvant chemoradiotherapy (nCRT) of locally advanced oesophageal cancer patients. Trials are ongoing which will prove whether organ preservation could be a safe alternative to surgery in patients with a clinical complete response. Crucial is the availability of tools that can accurately select those patients who will not be harmed by a non-operative treatment.

Our work has shown that DW-MRI as a single restaging modality after nCRT has a high sensitivity of $90-97 \%$ for the detection of residual tumour at the expense of a specificity of only $42-57 \%$ for the assessment of a tumour remnant. With only little experience in interpreting MRI of oesophageal cancer, the radiologists interpreting the DW-MRIs probably erred on the 'safe side' and overstaged rather than understaged in order to avoid undertreatment explaining the low specificity. The first studies on the value of DW-MRI for assessment of response to $\mathrm{nCRT}$ were in rectal cancer and these studies demonstrated specificities of restaging MRI in the same range (35-64\%) $)^{1,2}$. A rectal cancer study also demonstrated that there is a learning curve of approximately 50 scans $^{3}$. In rectal cancer, the combination of DW-MRI, rectal examination and endoscopy has proven the most accurate selection tool to identify patients with a clinical complete response $(\mathrm{CCR})^{2,4}$.

Several studies focused on endoscopy and EUS for the detection of residual tumour in oesophageal cancer and found that $28-55 \%$ of patients were incorrectly assessed as having cCR on endoscopy and EUS assessment ${ }^{5-8}$. The low sensitivity on endoscopy and EUS is probably caused by sampling error due to difficulties in identifying the right location to biopsy in areas of radiation-induced oesophagitis and ulcers. Complete response in the resection specimen did not always present as fibrosis at endoscopy and ulceration was not always an indication of residual tumour at histology (Chapter 5). Another explanation of the low sensitivity could be the presence of residual tumour cells beyond te mucosal wall layer in $28 \%$ of the patients 9 . On EUS the differentiation between tumour, fibrosis and inflammation remains challenging as there are no specific differentiating features ${ }^{10}$. An advantage of adding DW-MRI to endoscopy/EUS is the visualisation of the deeper wall layers and surrounding tissue as the field of view is substantially larger. Also DW-MRI can differentiate between fibrosis and malignancy as fibrosis does not restrict diffusion, while malignancy generally does. Chapter 5 found that the addition of DW-MRI to endoscopy and EUS with fine-needle aspiration (FNA) indeed led to a higher detection rate of patients with residual tumour, although these results should be interpreted with caution as the sample-size of this study was small, with only a limited amount of patients with pathological complete response (pCR). This was the first study on this subject and the promising results call for further research.

MRI of oesophageal cancer is promising but some challenges in the MR protocol need to be addressed. One challenge with DW-MRI is cardiac motion for tumours in the middle and distal oesophagus, gastric motion for tumours located in the distal oesophagus/gastro-oesophageal 
junction and breathing motion for all oesophageal tumours. The use of an anti-peristaltic agent (e.g. scopolamine- $\mathrm{N}$-butyl bromide) has been reported by some to be beneficial for reduction of the movement of the gastric wall during scan acquisition ${ }^{11,12}$. Gastro-oesophageal junction tumours pose a challenge as some patients with $\mathrm{pCR}$ show false-positive hyperintense foci on DW-MRI. This may be explained by the fact that collapsed normal stomach wall also can show hyperintensities on DW-MRI probably due to fluid 'trapped' in the folds. The administration of a negative oral contrast agent, for instance pineapple juice, is currently used for the visualisation of gastric cancer on MRI to suppress signal from physiologic stomach filling and might also improve imaging of gastrooesophageal junction tumours ${ }^{11,13,14}$.

Also, the optimal timeframe for response evaluation is yet unknown. Because the standard timing of surgery is 6-8 weeks after completion of $\mathrm{nCRT}^{15}$, the studies in this thesis generally performed response assessment 4-6 weeks after completion of $n C R T$. The DW-MRI in some patients may be 'false positive' at this early time point due to postradiation inflammation or, as has been observed in rectal cancer, tumours keep regressing with time until some eventually even completely disappear ${ }^{16,17}$. The 'test of time', i.e. a prolonged interval between completion of $\mathrm{nCRT}$ and response assessment, could be a good approach to solve confounding signals on DW-MRI. One could argue that false positive hyperintensities on DWI caused by postradiation inflammation may disappear and some tumours might have completely regressed. A nationwide analysis in the Netherlands showed that a prolonged interval ( $\geq 10$ weeks for adenocarcinomas and $\geq 13$ weeks for squamous cell carcinomas) between $\mathrm{nCRT}$ and surgery indeed increased the $\mathrm{PCR}$ rates ${ }^{18}$. Another advantage of the 'test of time' is the selection of patients with biologically aggressive tumours, that metastasize early. In $8 \%$ of the patients distant metastases are currently detected on FDG-PET/CT before oesophagectomy ${ }^{19,20}$. In patients having distant metastases, avoiding a futile surgical procedure with considerable associated morbidity may be desirable.

To date, FDG-PET/CT is the main modality for distant staging of oesophageal cancer. However, new imaging modalities such as whole-body DWI are rapidly evolving ${ }^{21-23}$. Previous studies have already demonstrated that DW-MRI is superior to FDG-PET/CT for the detection of liver metastases $^{24,25}$. In oesophageal cancer a similar accuracy of whole-body DWI compared to FDGPET/CT was reported ${ }^{22}$. In Chapter 4 DW-MRI and FDG-PET/CT were compared for locoregional response assessment after $\mathrm{nCRT}$. The $92-96 \%$ sensitivity for detecting residual oesophageal tumour after nCRT on DW-MRI was superior to the $69 \%$ of PET/CT. The low sensitivity of FDG-PET/CT may be explained by the difficulty of detecting glucose uptake on FDG-PET/CT in scattered residual tumour. Also, in areas of radiation oesophagitis one may understage tumour residue whereas DWMRI seems to suffer less heavily from inflammatory response. The accuracy of detecting lymph node metastases with DW-MRI (sensitivity 58\%-67\%; specificity $86 \%$ ) was comparable with FDG-PET/ CT (sensitivity $50 \%-42 \%$; specificity $81 \%-86 \%$ ). This was quite surprising as our hypothesis was 
that the differentiation between benign and malignant lymph nodes on DW-MRI would be more challenging than on FDG-PET/CT, as diffusion restriction is seen in both benign and malignant lymph nodes. Also, on MRI the field of view (FOV) is limited to the area around the oesophageal tumour whereas FDG-PET/CT visualises the whole mediastinal area. The high specificities on DWMRI suggest that the absence of suspect lymph nodes may yield predictive value for absence of nodal disease at histopathology. Previous literature about restaging of lymph nodes after nCRT on DW-MRI is scarce, but Riddell et al. compared N-staging on MRI to the surgical specimen and found a sensitivity of $64 \%$ and specificity of $79 \%{ }^{26}$. Although these results seem promising, for future studies a FOV of DW-MRI containing the complete mediastinal area is essential for accurate $\mathrm{N}$-staging. If it can be confirmed that DW-MRI is not inferior to FDG-PET/CT for locoregional and distant staging, whole-body DWI could potentially serve as a one stop shop imaging modality.

Meanwhile, the MR linear accelerator (MR-Linac) has entered clinical practice and MR guided radiotherapy treatment is increasingly explored for its potential advantages, such as reduced margins and plan adaptation which could reduce morbidity and increase treatment efficacy ${ }^{27,28}$. A considerable decrease in tumour volume was observed in oesophageal cancer patients during chemoradiotherapy treatment ${ }^{29}$. In Chapter 6 we found that delineation of the radiotherapy target region on DW-MRI is feasible. Plan adaptation and radiation dose escalation on the MR-Linac may increase the amount of complete responders. This is especially true for squamous cell carcinomas which are known to be more radiosensitive than adenocarcinomas ${ }^{6}$. A randomized-controlled multicenter study compared definitive chemoradiotherapy of 61.6 Gray to the standard 50.4 Gray and found no increased local control nor better overall survival ${ }^{30}$. However, future studies should explore if dose escalation in patients with predicted good response to nCRT results in an increase of complete responders. To select patients with a high chance of achieving a complete response the relative difference in apparent diffusion coefficient (ADC) derived from DW-MRI before vs. during nCRT as shown in Chapter 3 as well as circulating tumour DNA (ctDNA) may be useful ${ }^{31-34}$. These biomarkers and especially their integration should be the subject of future studies.

The introduction of neoadjuvant chemoradiotherapy for patients with locally advanced oesophageal cancer has led to new diagnostic challenges. A point of discussion is whether a select group of patients with complete response after chemoradiotherapy could be deferred from surgery. Careful selection of patients for watchful waiting is crucial. The research presented in this thesis found promising results for DW-MRI as part of a multimodality response assessment approach. DWMRI added accuracy to the restaging of oesophageal cancer by endoscopy/EUS examination and for both local tumour and regional nodal restaging DW-MRI competes with FDG-PET/CT. The promising outcomes of the studies reported in this thesis contributed to the initiation of the multicentre PRIDE study, which is currently exploring the role of MRI, FDG-PET/CT, ctDNA and endoscopy/EUS in response prediction and assessment of oesophageal cancer ${ }^{35}$. At the same 
time, the Dutch SANO trial ${ }^{36}$ and the French ESOSTRATE trial ${ }^{37}$ are comparing a watch-and-wait approach to surgery for clinical complete responders after neoadjuvant chemoradiotherapy. In the coming years, future studies should further investigate which is the most accurate combination of modalities for selection of clinical complete responders, what would be the optimal timing of response assessment and which would be the most cost-efficient follow-up schedule to move forward the paradigm shift towards watchful waiting in oesophageal cancer therapy. 


\section{REFERENCES}

1. Lambregts DM, Vandecaveye V, Barbaro B, et al. Diffusion-weighted MRI for selection of complete responders after chemoradiation for locally advanced rectal cancer: a multicenter study. Ann Surg Oncol $2011 ; 18: 2224-31$.

2. Maas M, Lambregts DM, Nelemans PJ, et al. Assessment of Clinical Complete Response After Chemoradiation for Rectal Cancer with Digital Rectal Examination, Endoscopy, and MRI: Selection for Organ-Saving Treatment. Ann Surg Oncol 2015;22:3873-80.

3. Lambregts DMJ, van Heeswijk MM, Delli Pizzi A, et al. Diffusion-weighted MRI to assess response to chemoradiotherapy in rectal cancer: main interpretation piffalls and their use for teaching. Eur Radiol 2017;27:4445-54.

4. Lambregts DM, Rao SX, Sassen S, et al. MRI and Diffusion-weighted MRI Volumetry for Identification of Complete Tumor Responders After Preoperative Chemoradiotherapy in Patients With Rectal Cancer: A Bi-institutional Validation Study. Ann Surg 2015;262:1034-9.

5. Castoro C, Scarpa M, Cagol $M$, et al. Complete clinical response after neoadjuvant chemoradiotherapy for squamous cell cancer of the thoracic oesophagus: is surgery always necessary? J Gastrointest Surg 2013;17:137581.

6. Noordman BJ, Spaander MCW, Valkema $R$, et al. Detection of residual disease after neoadjuvant chemoradiotherapy for oesophageal cancer (preSANO): a prospective multicentre, diagnostic cohort study. Lancet Oncol 2018;19:965-74.

7. Furlong $H$, Bass $G$, Breathnach $O, O^{\prime}$ Neill $B$, Leen E, Walsh TN. Targeting therapy for esophageal cancer in patients aged 70 and over. J Geriatr Oncol 2013;4:107-13.

8. Ohkura $Y$, Shindoh J, Ueno $M$, lizuka $T$, Udagawa $H$. Comparison of Outcome of Esophagectomy Versus Nonsurgical Treatment for Resectable Esophageal Cancer with Clinical Complete Response to Neoadjuvant Therapy. Ann Surg Oncol 2018;25:2428-33.

9. Shapiro J, ten Kate FJ, van Hagen P, Biermann $\mathrm{K}$, Wijnhoven BP, van Lanschot JJ. Residual esophageal cancer after neoadjuvant chemoradiotherapy frequently involves the mucosa and submucosa. Ann Surg 2013;258:678-88; discussion 88-9.

10. Griffin JM, Reed CE, Denlinger CE. Utility of restaging endoscopic ultrasound after neoadjuvant therapy for esophageal cancer. Ann Thorac Surg 2012;93:1855-9; discussion 60.

11. Borggreve AS, Goense L, Brenkman HJF, et al. Imaging strategies in the management of gastric cancer: current role and future potential of MRI. Br J Radiol 2019;92:20181044.

12. Giganti F, Tang L, Baba H. Gastric cancer and imaging biomarkers: Part 1 - a critical review of DW-MRI and CE-MDCT findings. Eur Radiol 2019;29:1743-53.

13. Alshehri FM. Comparative study of pineapple juice as a negative oral contrastagent in magnetic resonance cholangiopancreatography. J Clin Diagn Res 2015;9:TC13-6.

14. Pan D, Schmieder AH, Wickline SA, Lanza GM. Manganese-based MRI contrast agents: past, present and future. Tetrahedron $2011 ; 67: 8431$ 44.

15. van Hagen $P$, Hulshof MC, van Lanschot JJ, et al. Preoperative chemoradiotherapy for esophageal or junctional cancer. N Engl J Med 2012;366:2074-84.

16. Sloothaak DA, Geijsen DE, van Leersum NJ, et al. Optimal time interval between neoadjuvant chemoradiotherapy and surgery for rectal cancer. Br J Surg 2013;100:933-9.

17. West MA, Dimitrov BD, Moyses HE, et al. Timing of surgery following neoadjuvant chemoradiotherapy in locally advanced rectal cancer - A comparison of magnetic resonance imaging at two time points and histopathological responses. Eur J Surg Oncol 2016;42:1350-8.

18. van der Werf LR, Dikken JL, van der Willik $E M$, et al. Time interval between neoadjuvant chemoradiotherapy and surgery for oesophageal or junctional cancer: A nationwide study. Eur J Cancer 2018;91:76-85.

19. Stiekema J, Vermeulen $D$, Vegt $E$, et al. Detecting interval metastases and response assessment 
using 18F-FDG PET/CT after neoadjuvant chemoradiotherapy for esophageal cancer. Clin Nucl Med 2014;39:862-7.

20. Goense L, Ruurda JP, Carter BW, et al Prediction and diagnosis of interval metastasis after neoadjuvant chemoradiotherapy for oesophageal cancer using (18)F-FDG PET/CT. Eur J Nucl Med Mol Imaging 2018;45:174251.

21. Gong J, Cao W, Zhang Z, et al. Diagnostic efficacy of whole-body diffusion-weighted imaging in the detection of fumour recurrence and metastasis by comparison with 18F-2fluoro-2-deoxy-D-glucose positron emission tomography or computed tomography in patients with gastrointestinal cancer. Gastroenterol Rep 2015;3:128-35.

22. Malik V, Harmon M, Johnston C, et al. Whole Body MRI in the Staging of Esophageal Cancer-A Prospective Comparison with Whole Body 18F-FDG PET-CT. Dig Surg 2015;32:397-408.

23. Yoon $J H, Y u M H$, Hur BY, Park $C M$, Lee $J M$. Detection of distant metastases in rectal cancer: contrast-enhanced CT vs whole body MRI. Eur Radiol 2020.

24. Niekel MC, Bipat S, Stoker J. Diagnostic imaging of colorectal liver metastases with CT, $M R$ imaging, FDG PET, and/or FDG PET/CT: a meta-analysis of prospective studies including patients who have not previously undergone treatment. Radiology 2010;257:674-84.

25. Sivesgaard $K$, Larsen $L P$, Sorensen $M$, et al. Diagnostic accuracy of CE-CT, MRI and FDG $\mathrm{PET} / \mathrm{CT}$ for detecting colorectal cancer liver metastases in patients considered eligible for hepatic resection and/or local ablation. Eur Radiol 2018;28:4735-47.

26. 26. Riddell $A M$, Allum $W H$, Thompson $J N$, Wotherspoon AC, Richardson C, Brown G. The appearances of oesophageal carcinoma demonstrated on high-resolution, T2-weighted MRI, with histopathological correlation. Eur Radiol 2007;17:391-9.

27. Kerkmeijer LG, Fuller CD, Verkooijen HM, et al. The MRI-Linear Accelerator Consortium: Evidence-Based Clinical Introduction of an Innovation in Radiation Oncology Connecting Researchers, Methodology, Data Collection, Quality Assurance, and Technical Development. Front Oncol 2016;6:215.
28. Schultz C. MR-linac: Clinical introduction. Radiother Oncol 2016;119:S232.

29. Defize IL, Boekhoff MR, Borggreve AS, et al. Tumor volume regression during neoadjuvant chemoradiotherapy for esophageal cancer: a prospective study with weekly MRI. Acta Oncol 2020;59:753-9.

30. Hulshof MCCM, Geijsen D, Rozema T, et al. A randomized controlled phase III multicenter study on dose escalation in definitive chemoradiation for patients with locally advanced esophageal cancer: ARTDECO study. J Clin Oncol 2020;38:281-281.

31. Sclafani F, Smyth E, Cunningham D, Chau I, Turner A, Watkins D. A pilot study assessing the incidence and clinical significance of circulating tumor cells in esophagogastric cancers. Clin Colorectal Cancer 2014;13:94-9.

32. Creemers A, Krausz $S$, Strijker $M$, et al. Clinical value of ctDNA in upper-Gl cancers: $A$ systematic review and meta-analysis. Biochim Biophys Acta Rev Cancer 2017; 1868:394-403.

33. Gopalan V, Lam AK. Circulatory Tumor Cells in Esophageal Adenocarcinoma. Methods Mol Biol 2018;1756:177-86.

34. Azad TD, Chaudhuri AA, Fang P, etal. Circulating Tumor DNA Analysis for Detection of Minimal Residual Disease After Chemoradiotherapy for Localized Esophageal Cancer. Gastroenterology 2020;158:494-505 e6.

35. Borggreve AS, Mook $S$, Verheii $M$, et al. Preoperative image-guided identification of response to neoadjuvant chemoradiotherapy in esophageal cancer (PRIDE): a multicenter observational study. BMC Cancer 2018;18:1006.

36. Noordman BJ, Wijnhoven BPL, Lagarde $S M$, et al. Neoadjuvant chemoradiotherapy plus surgery versus active surveillance for oesophageal cancer: a stepped-wedge cluster randomised trial. BMC Cancer 2018;18:142.

37. clinicaltrials.gov [internet]. Centre Hospitalier Universitaire Dijon: Comparison of Systematic Surgery Versus Surveillance and Rescue Surgery in Operable Oesophageal Cancer With a Complete Clinical Response to Radiochemotherapy (Esostrate) NCT02551458 2015 [updated 1/12/16]. Available: https:// clinicaltrials.gov/show/NCT02551458. 


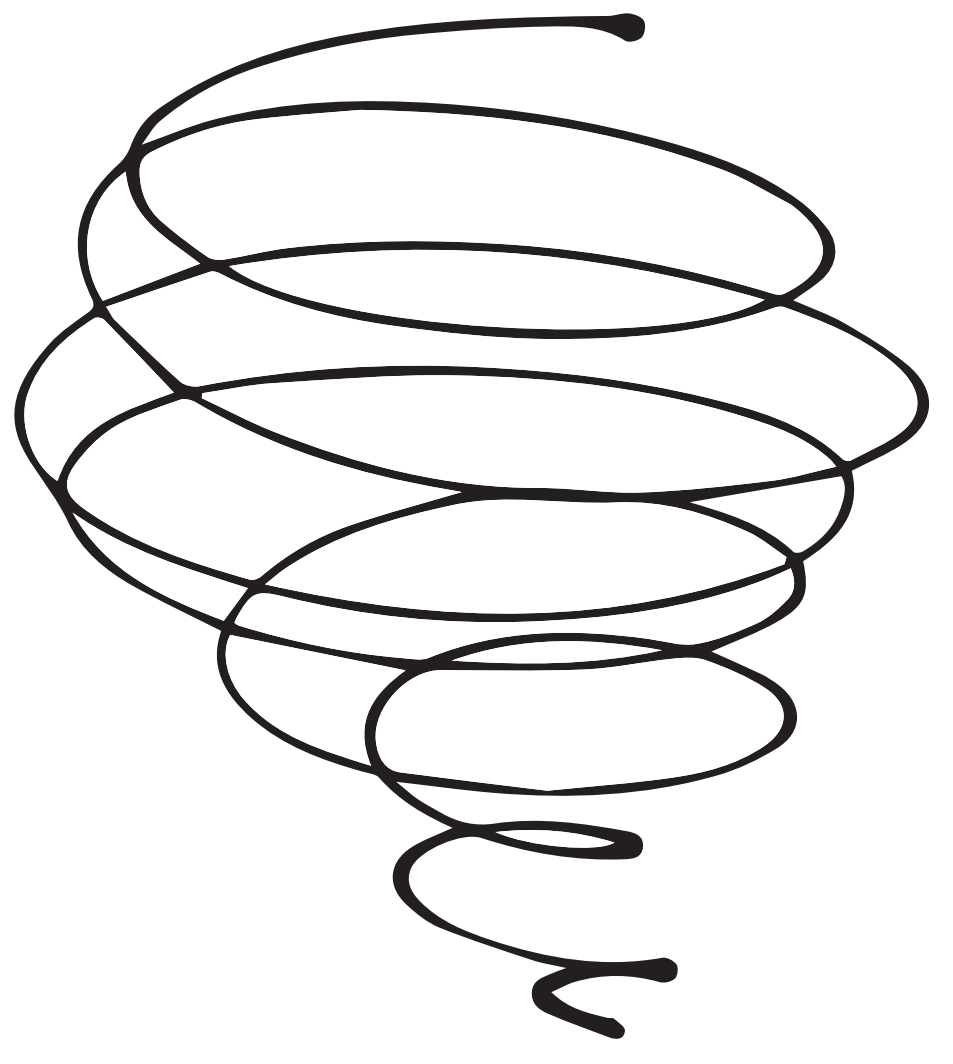


APPENDICES

Summary 
Locally advanced oesophageal cancer patients are currently treated by chemoradiotherapy followed by surgery. In $29 \%$ of the patients, no residual cancer cells are detected in the resected specimen. These patients are called 'complete responders'. Accurate response assessment after chemoradiotherapy is likely to impact clinical decision making, as many experts state that patients with a clinical complete response could be deferred from surgery. The aim of this thesis was to investigate whether diffusion-weighted MRI (DW-MRI) is able to improve the clinical selection after chemoradiotherapy of patients who are eligible for organ preservation. Also, the potential for MRI in radiotherapy treatment planning was investigated.

The visual response assessment on DW-MRI by three oncologically specialised radiologists in 51 patients was explored in Chapter 2. A high sensitivity for the detection of residual tumour was found, implicating a low risk of missing complete responders. At the same time, the specificity was low, which means that the risk of overstaging a patient with a complete response was considerable. Thus with subjective response evaluation, radiologists tended to err on the "safe side" and rather overstaged than understaged the primary oesophageal tumour after chemoradiotherapy.

From DW-MRI the apparent diffusion coefficient (ADC) can be calculated. The ADC is a quantitative, and thereby potentially objective, measurement of the diffusivity of water molecules in tissues. In Chapter 3 the value of quantitative DW-MRI was explored by performing a systematic review. Fourteen studies comprising 516 patients calculated the ADC before, during and after chemoradiotherapy. It was found that the relative increase in $A D C$ (delta $A D C$ ) during, compared to before chemoradiotherapy, can potentially differentiate between good and poor responders. For response assessment after chemoradiotherapy, ADC values seem inadequate because the region of interest size and placement is hampered by small or absent tumour in good/complete responders. Therefore, response after chemoradiotherapy may rather be assessed visually.

Another widely used cancer imaging technique is computed tomography combined with 18F-fluorodeoxyglucose positron emission tomography (FDG-PET/CT). Although FDG-PET/CT is used for the staging of distant metastases and lymph nodes in oesophageal cancer, it is also increasingly explored for its role in local restaging after chemoradiotherapy. Chapter 4 compared DW-MRI to FDG-PET/CT for this purpose in 33 patients and it was found that MRI resulted in a more accurate response assessment as compared to FDG-PET/CT. The most important finding was the higher sensitivity on DW-MRI as compared to FDG-PET/CT.

If DW-MRI can become the main imaging modality in response assessment of oesophageal cancer after chemoradiotherapy, it is important to understand its potential benefit to endoscopy and endoscopic ultrasound (EUS). Previous studies found that a considerable proportion of residual tumours is currently missed based on the biopsies and aspirates taken by endoscopy and EUS. 
In Chapter 5 the addition of DW-MRI to endoscopy/EUS with fine needle aspiration (FNA) led to a higher detection rate of patients with residual tumour in 22 patients. It was also found that the clinical perception of the gastroenterologist did not add to the pathological assessment of biopsies: complete response neither always presented as residual fibrosis, nor did ulceration always implicate residual tumour at 4 to 6 weeks after chemoradiotherapy.

Simultaneously, the development of an MRI-scanner with linear accelerator for radiotherapy has led to the possibility of MRI guidance of radiotherapy. Potential advantages of MRI guidance are reduced margins, plan adaptation and reducing the fractions of radiotherapy. In Chapter 6, ten observers delineated the radiotherapy target region of the primary oesophageal tumour in six patients on DW-MRI, using FDG-PET/CT as the reference standard. Delineation on DW-MRI was feasible with interobserver variability comparable to that of FDG-PET/CT.

To conclude, by exploring the role of DW-MRI in a multimodal approach, this thesis contributes to the movement towards organ saving treatment for locally advanced oesophageal cancer patients with a clinical complete response to chemoradiotherapy. 


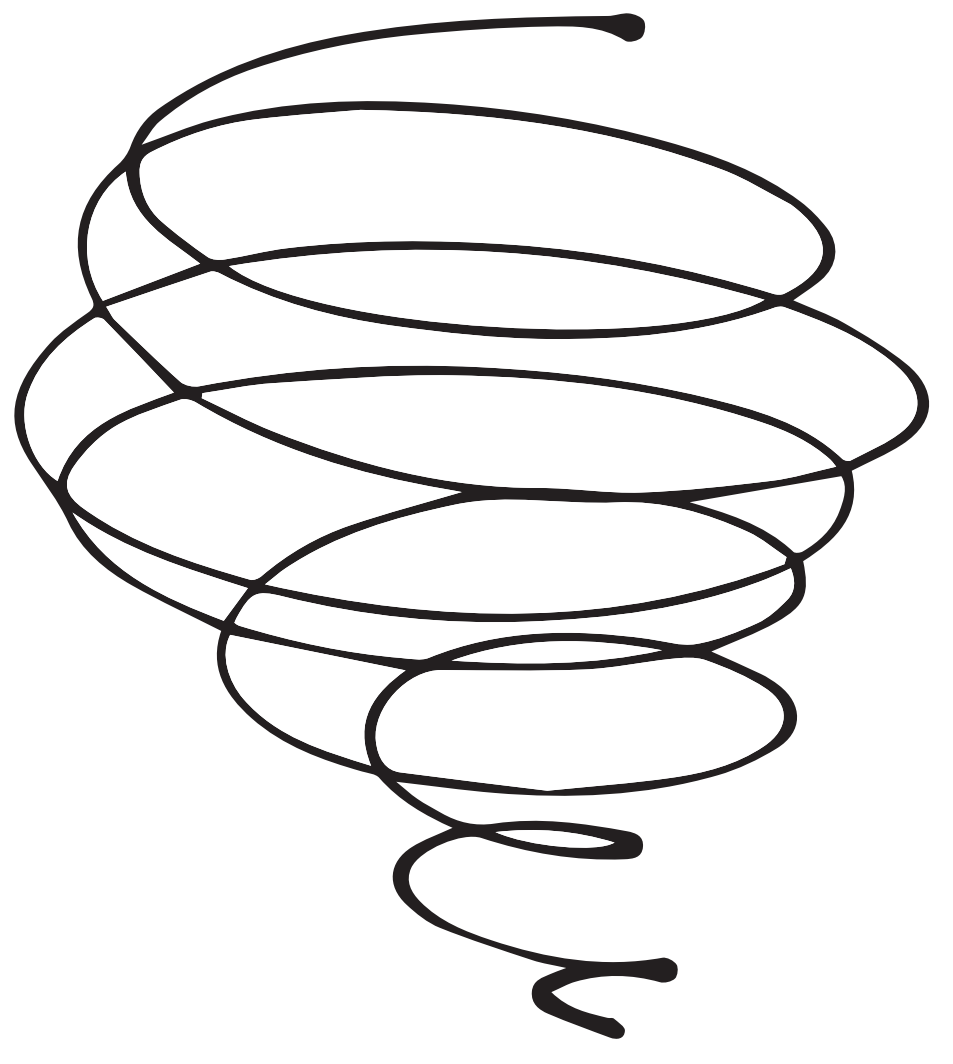


APPENDICES

Samenvatting 
Patiënten met lokaal gevorderde slokdarmkanker worden op dit moment behandeld met chemoradiatie gevolgd door een operatie. Na de operatie vindt de patholoog in $29 \%$ van de patiënten geen tumorcellen meer in de slokdarm, dit wordt ook wel een 'complete respons' genoemd. Bij deze patiënten wordt overwogen om de chirurgie na chemoradiatie achterwege te laten: orgaansparende behandeling. Om te bepalen wie in aanmerking komt voor orgaansparende behandeling na chemoradiatie is een goede selectiemethode belangrijk.

Technologische ontwikkelingen hebben ervoor gezorgd dat het mogelijk is om goede afbeeldingen van de slokdarm te maken met magnetic resonance imaging (MRI). MRI biedt tevens de mogelijkheid om functionele beeldvorming, zoals diffusie-gewogen MRI, te combineren met anatomische beelden (DW-MRI). In dit proefschrift wordt onderzocht of DW-MRI de selectie van patiënten voor orgaansparende behandeling na chemoradiatie kan verbeteren en of het veilig is om DW-MRI voor het bestralingsplan van slokdarmkanker te gebruiken.

In Hoofdstuk 2 wordt de aanwezigheid van tumor op DW-MRI na chemoradiatie onderzocht door 3 onafhankelijke radiologen in 51 patiënten. Er werd een hoge sensitiviteit voor de detectie van residuele tumoren gevonden, wat betekent dat er een laag risico was om residuele tumoren te missen. Tegelijkertijd was de specificiteit laag, dus er was wel een relatief grote kans op het overstadieren van complete respons.

Vanuit de DW-MRI kun je een kwantitatieve waarde berekenen: de apparent diffusion coefficient (ADC). Dit is een objectieve parameter voor de diffusie van watermoleculen in weefsels. In Hoofdstuk 3 wordt de waarde van ADC onderzocht in een systematisch literatuuronderzoek. Veertien artikelen met 516 patiënten werden geïncludeerd. De relatieve stijging van ADC (delta ADC) tijdens chemoradiatie vergeleken met vóór start van chemoradiatie lijkt onderscheid te kunnen maken tussen goede en slechte responders. Voor de waarde van ADC voor restadiëring na neo-adjuvante chemoradiatie, liepen de resultaten viteen. Dit komt waarschijnlijk doordat het moeilijk is om te bepalen in welk gebied de ADC-meting gedaan moet worden als een slokdarm geen, of bijna geen, residueel tumorgebied meer heeft.

Voor het bestralingsplan wordt gebruik gemaakt van 18F-fluorodeoxyglucose positron emission tomography - computed tomography (18F-FDG-PET/CT) om de precieze locatie en vorm van de tumor in te tekenen. Ook wordt FDG-PET/CT in steeds grotere mate onderzocht voor de selectie van complete responders na chemoradiatie. In Hoofdstuk 4 worden DW-MRI en FDG-PET/CT vergeleken voor de lokale restadiëring van de slokdarmtumor in 33 patiënten. DW-MRI resulteerde in een betere responsbeoordeling vergeleken met FDG-PET/CT, waarbij voornamelijk de sensitiviteit op DW-MRI hoger was dan op de FDG-PET/CT. 
Met behulp van een endoscoop kan de maag-darm-lever arts (MDL-arts) de slokdarm beoordelen en biopten afnemen. De endoscopische echo (EUS) kan hieraan worden toegevoegd: middels echo wordt vanuit het lumen van de slokdarm door de wand van de slokdarm heen gekeken. Met behulp van EUS kunnen aanvullend puncties van lymfeklieren genomen worden. Eerdere onderzoeken suggereren dat er met endoscopie/EUS na chemoradiatie een hoge kans is op vals-negatieven. Indien dit gebruikt wordt voor de selectie van complete responders, zouden patiënten met residu tumor mogelijk onterecht worden onthouden van de operatie. In Hoofdstuk 5 werd de aanvullende waarde van DW-MRI bij endoscopie/EUS na chemoradiatie onderzocht in 22 patiënten. Alle patiënten ondergingen een MRI-scan en endoscopie/EUS onderzoek vóór en na chemoradiatie. De aanvullende beoordeling van de radioloog op DW-MRI aan de biopten en puncties van endoscopie/EUS leidde tot een hogere detectie van patiënten met residuele tumor. Daarnaast bleek het lastig voor de MDL-arts om een klinische inschatting te maken van de verdenking op residuele tumor op basis van visuele beoordeling tijdens het endoscopie/EUS-onderzoek.

Tegelijkertijd heeft de ontwikkeling van een MRI met lineaire versneller voor radiotherapie geleid tot de mogelijkheid van MRI-begeleiding tijdens de bestraling. Mogelijke voordelen van MRI-begeleiding zijn verminderde marges, directe aanpassing van het bestralingsplan en het verminderen van de fracties van radiotherapie. Omdat MRI op dit moment nog niet wordt gebruikt voor het bestralingsplan van slokdarmkanker is het allereerst belangrijk om te kijken of het veilig zou zijn om MRI te gebruiken voor het intekenen van slokdarmkanker (Hoofdstuk 6). Tien artsen hebben de slokdarmtumor ingetekend op PET-CT en op MRI en er werd een vergelijkbare interobserver-variatie voor beide modaliteiten gevonden. Hoewel verder onderzoek in een grotere groep nodig is, waren de resultaten veelbelovend.

Concluderend werd in dit proefschrift de rol van DW-MRI onderzocht voor de selectie van patiënten die een orgaansparende behandeling zouden kunnen ondergaan na chemoradiatie. Daarmee draagt dit proefschrift bij aan de ontwikkeling van orgaansparende behandeling voor patiënten met slokdarmkanker en een complete respons na chemoradiatie. 


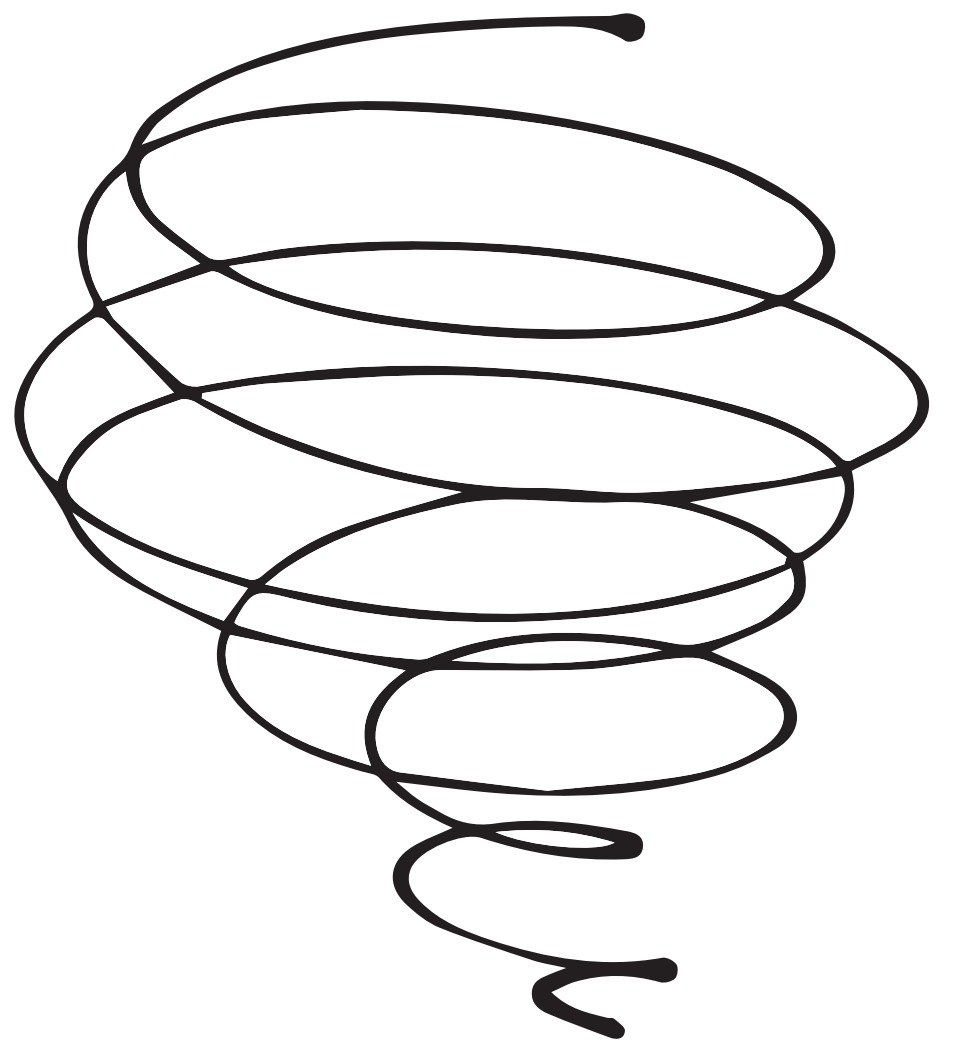


APPENDICES

Valorisation 
In this chapter, the thesis will be analysed in the perspective of the valorisation focusing on its relevance for society and clinics. Also, the novelty and future realisation will be outlined. The following five aspects will be discussed:

1. Socio-economic relevance

2. Target audience

$3 . \quad$ Products

4. Innovative value

5. Realisation

\section{Socio-economic relevance}

Cancer has a major impact on society across the world and oesophageal cancer is in the top ten of most common cancer types worldwide. Especially the incidence of adenocarcinomas in men is growing rapidly. One of the major risk factors for oesophageal adenocarcinomas is gastrooesophageal reflux disease (GORD) causing metaplasia of the distal oesophagus. Obese or overweight individuals are significantly more at risk of suffering from GORD. In 2016,39\% men and $39 \%$ women aged $18+$ were overweight $\left(B M I>25 \mathrm{~kg} / \mathrm{m}^{2}\right)$ and $11 \%$ of men and $15 \%$ of women were obese $\left(\mathrm{BMl}>30 \mathrm{~kg} / \mathrm{m}^{2}\right)$.

Patients diagnosed with locally advanced oesophageal cancer are currently treated by neoadjuvant chemoradiotherapy followed by surgery in the Netherlands. A personalised treatment approach is increasingly explored in many cancer types and could also be interesting for oesophageal cancer patients. Previous analyses showed that in almost $30 \%$ of the patients no residual cancer cells were found in the surgical specimen, a phenomenon which is called a "pathological complete response". These patients might benefit from an organ saving treatment approach after 'neoadjuvant' chemoradiotherapy. This thesis outlines the role for MRI in a multimodal diagnostic approach after chemoradiotherapy to open the door to organ preservation for clinical complete responders. Also, the feasibility for MRI was investigated at the start of treatment for the delineation of the target volume.

\section{Target population}

Following national guidelines, patients with oesophageal cancer are currently treated by chemoradiotherapy followed by surgery. This thesis investigates a multimodal diagnostic approach to select patients for organ preserving treatment after chemoradiotherapy. Therefore, the results of this thesis may primarily impact the treatment plan of oesophageal cancer patients. Omitting surgery may likely impact their lives because oesophageal surgery is reported to have a major impact on patients' quality of life. Interestingly, a study in rectal cancer reported promising improvement of the quality of life after chemoradiotherapy in the watch-and-wait group compared with the surgery group. If surgery is to be omitted in oesophageal cancer patients with a clinical complete response after chemoradiotherapy, future studies should explore the quality of life as well 
as the cost efficiency of this new treatment.

The results in this thesis may also impact the work of the multidisciplinary team of oesophageal cancer patients. The assessment of MRI by the radiologist will probably play a prominent role in the response assessment after chemoradiotherapy. Furthermore, implementing an organ preserving treatment approach may impact the numbers of performed operations, while this number is currently an important requirement for quality assessment in the Netherlands.

\section{Products}

There are no new products that have been developed with the results of this thesis.

Innovative value

An organ-preserving treatment approach is upcoming in other cancer sites and the promising response rates to chemoradiotherapy opens the debate for oesophageal cancer patients. To select the right patients for organ preservation, accurate response assessment is essential. The high rates of false negative biopsies taken by endoscopy stresses the need for an accurate additional imaging tool. MRI is increasingly explored for this purpose because of the good visualisation of soft tissues combined with functional diffusion-weighted imaging. This thesis reports new and relevant studies on the clinical assessment on MRI in oesophageal cancer and thereby contributes to the development of a personalised treatment approach for oesophageal cancer patients.

\section{Realisation}

Currently, the multicentre PRIDE trial is exploring the combination of MRI, FDG-PET/CT, endoscopy/ EUS and circulating tumour DNA for predicting and assessing the response to chemoradiotherapy in oesophageal cancer. At the same time, two trials are exploring a watch-and-wait approach for patients with oesophageal cancer: the SANO trial in the Netherlands and the ESOSTRATE trial in France. The combination of the aforementioned studies will undoubtedly lead to a more personalised treatment approach for patients with locally advanced oesophageal cancer. 


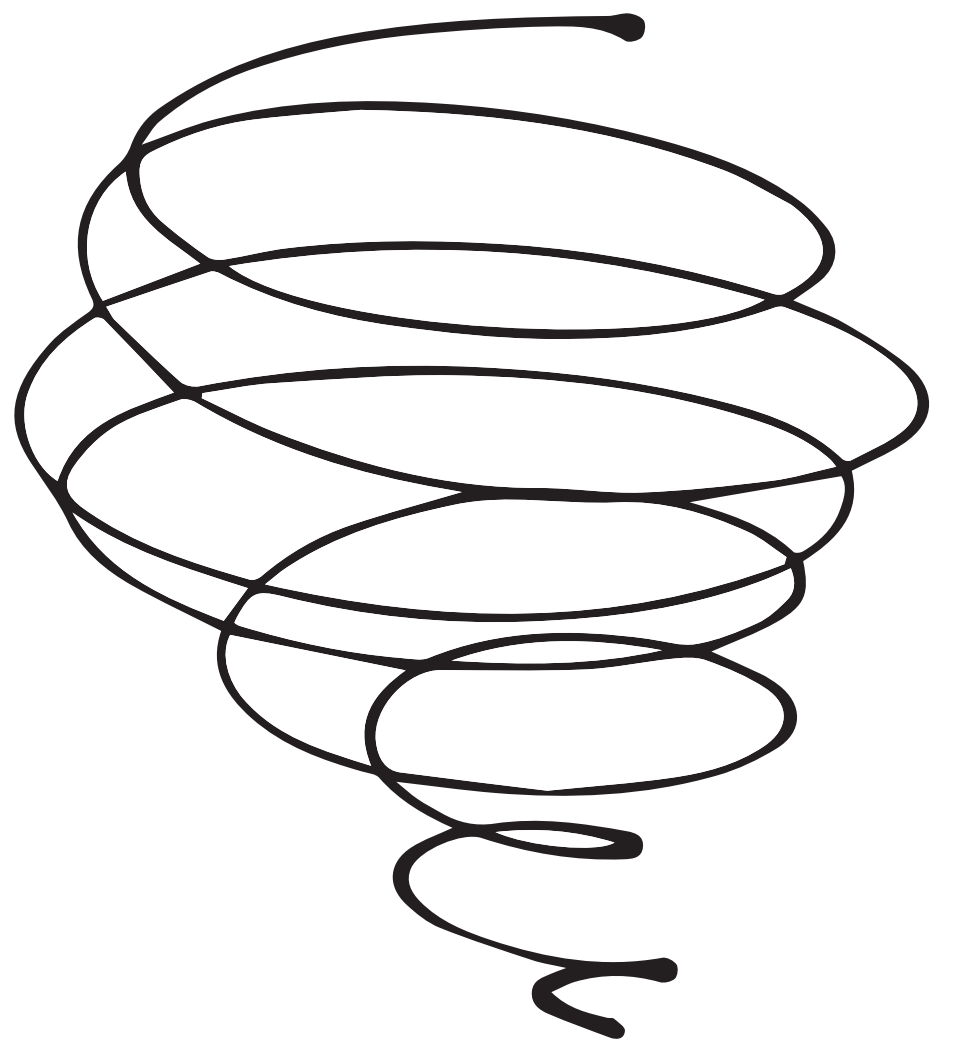




\section{APPENDICES}

\section{List of Publications}


Vollenbrock SE, Voncken FEM, Lambregts DMJ, Maas M, Vegt E, Donswijk M, ter Beek LC, van Sandick JW, Hartemink KJ, Aleman BMP, Beets-Tan RGH, Bartels-Rutten A.

Clinical response assessment on DW-MRI compared with FDG-PET/CT after neoadjuvant chemoradiotherapy in patients with oesophageal cancer.

Eur J Nucl Mol Imaging. 2020 June 22. Online, ahead of print.

Vollenbrock SE, van Dieren JM, Voncken FEM, van Turenhout ST, Kodach LL, van Sandick JW, Aleman BMP, Beets-Tan RGH, Bartels-Rutten A.

Added value of MRI to endoscopic and endosonographic response assessment after neoadjuvant chemoradiotherapy in oesophageal cancer.

Eur Radiol. 2020 May;30(5):2425-2434.

Voncken FEM, Nakhaee S, Stam B, Wiersema L, Vollenbrock SE, van Dieren JM, van Leerdam M, Sonke JJ, Aleman BMP, Remeijer P.

Quantification of oesophageal tumour motion and investigation of different image guided correction strategies.

Practical Radiat Oncol. 2020 Mar-Apr;10(2):84-92.

Vollenbrock SE, Voncken FEM, Bartels LW, Beets-Tan RGH, Bartels-Rutten A.

Diffusion-weighted MRI with ADC mapping for response prediction and assessment of oesophageal cancer: a systematic review.

Radiother Oncol. 2020 Jan;142:17-26.

Vollenbrock SE, Nowee ME, Voncken FEM, Kotte ANTJ, van Rossum PSN, van Lier ALHMW, Heijmink SW, Bartels-Rutten A, Wessels FJ, Aleman BMP, Dewit L, Kerkmeijer LGW, Jansen EPM, Intven M, Lips IM, Meijer GJ, Nijkamp J.

Gross tumor delineation in esophageal cancer on MRI compared to FDG-PET/CT.

Adv Radiat Oncol. 2019 Apr 24;4(4):596-604.

Vollenbrock SE, Voncken FEM, van Dieren JM, Lambregts DMJ, Maas M, Meijer GJ, Goense L, Mook S, Hartemink KJ, Snaebjornsson P, ter Beek LC, Verheij M, Aleman BMP, Beets-Tan RGH, Bartels-Rutten A.

Diagnostic performance of MRI for the assessment of response to neoadjuvant chemoradiotherapy in oesophageal cancer.

Br J Surg. 2019 Apr;106(5):596-605.

Borggreve AS, Mook S, Verheii M, Mul VEM, Bergman JJ, Bartels-Rutten A, ter Beek LC, Beets-Tan RGH, Bennink RJ, van Berge Henegouwen MI, Brosens LAA, Defize IL, van Dieren JM, Dijkstra 
$H$, van Hillegersberg R, Hulshof MC, van Laarhoven HWM, Lam MGEH, van Lier ALHMW, Muijs CT, Nagengast WB, Nederveen AJ, Noordzij W, Plukker JTM, van Rossum PSN, Ruurda JP, van Sandick JW, Weusten BLAM, Voncken FEM, Yakar D, Meijer GJ.

Collaborators: Aleman BMP, Borra RJH, van Etten B, Gisbertz SS, Goense L, Haj Mohammed N, Hartemink KJ, Kappert P, Kats-Ugurlu G, Kodach LL, Korteweg T, Krishnadath KK, Langendiik JA, de Leng WWJ, Meijer SL, Potze JH, Stoker J, Vegt E, Verkooijen HM, Vollenbrock SE, Wessels F. Preoperative image-guided identification of response to neoadjuvant chemoradiotherapy in esophageal cancer (PRIDE): a multicenter observational study.

BMC Cancer. 2018;18(1):1006. 


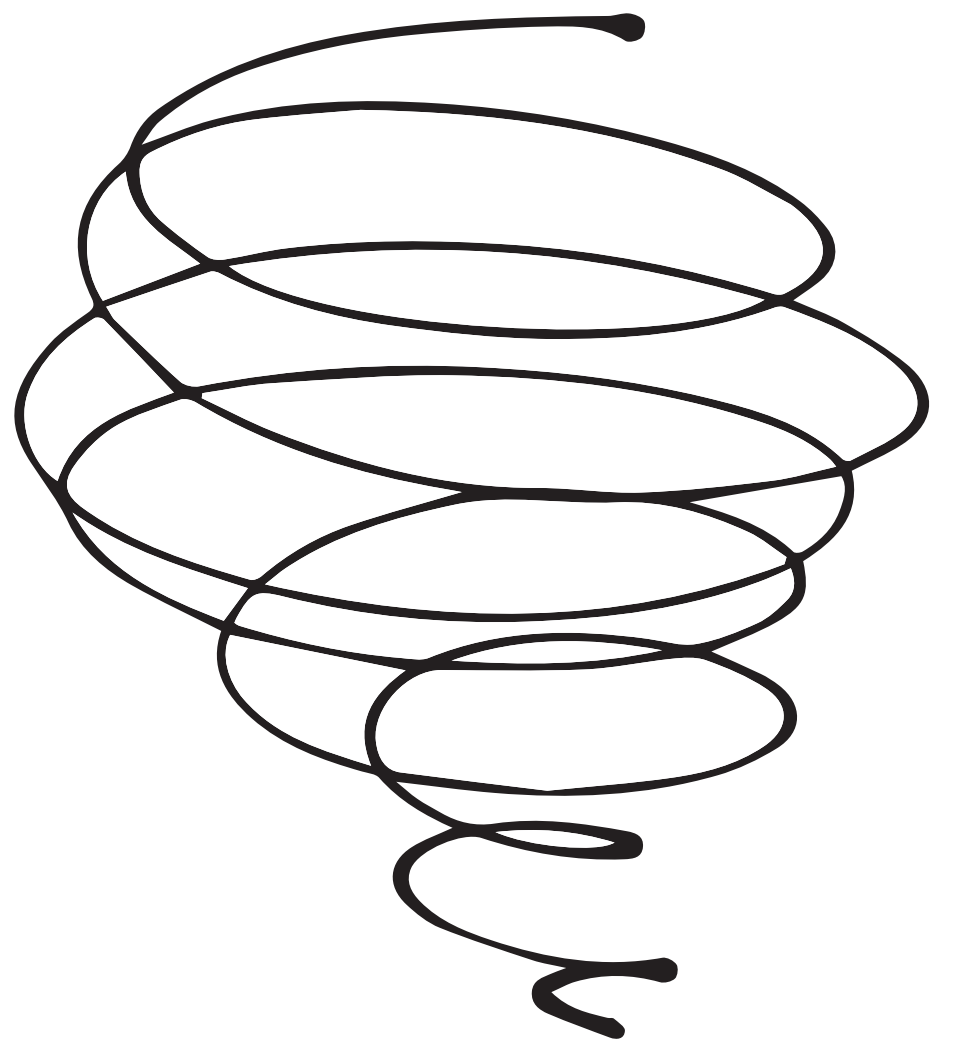




\section{APPENDICES}

List of Contributing Authors 


\section{B.M.P. Aleman}

Department of Radiation Oncology

Antoni van Leeuwenhoek

The Netherlands Cancer Institute

Amsterdam, the Netherlands

L.W. Bartels

Image Sciences Institute, Imaging Division

University Medical Center Utrecht

Utrecht, the Netherlands

A. Bartels-Rutten

Department of Radiology

Antoni van Leeuwenhoek

The Netherlands Cancer Institute

Amsterdam, the Netherlands

L.C. ter Beek

Department of Radiology

Antoni van Leeuwenhoek

The Netherlands Cancer Institute

Amsterdam, the Netherlands

R.G.H. Beets-Tan

Department of Radiology

Antoni van Leeuwenhoek

The Netherlands Cancer Institute

Amsterdam, the Netherlands

L. Dewit

Department of Radiation Oncology

Antoni van Leeuwenhoek

The Netherlands Cancer Institute

Amsterdam, the Netherlands

J.M. van Dieren

Department of Gastroenterology

Antoni van Leeuwenhoek
The Netherlands Cancer Institute

Amsterdam, the Netherlands

M.L. Donswijk

Department of Nuclear Medicine

Antoni van Leeuwenhoek

The Netherlands Cancer Institute

Amsterdam, the Netherlands

L.. Goense

Department of Surgery and Radiation

Oncology

University Medical Center Utrecht

Utrecht, the Netherlands

K.J. Hartemink

Department of Surgery

Antoni van Leeuwenhoek

The Netherlands Cancer Institute

Amsterdam, the Netherlands

S.W. Heijmink

Department of Radiology

Antoni van Leeuwenhoek

The Netherlands Cancer Institute

Amsterdam, the Netherlands

M. Intven

Department of Radiation Oncology

University Medical Center Utrecht

Utrecht, the Netherlands

E.P.M. Jansen

Department of Radiation Oncology

Antoni van Leeuwenhoek

The Netherlands Cancer Institute

Amsterdam, the Netherlands 
L.G.W. Kerkmeijer

Department of Radiation Oncology

University Medical Center Utrecht

Utrecht, the Netherlands

\section{L.L. Kodach}

Department of Pathology

Antoni van Leeuwenhoek

The Netherlands Cancer Institute

Amsterdam, the Netherlands

A.N.T.J. Kotte

Department of Radiation Oncology

University Medical Center Utrecht

Utrecht, the Netherlands

D.M.J. Lambregts

Department of Radiology

Antoni van Leeuwenhoek

The Netherlands Cancer Institute

Amsterdam, the Netherlands

A.L.H.M.W. van Lier

Department of Radiation Oncology

University Medical Center Utrecht

Utrecht, the Netherlands

I.M. Lips

Department of Radiation Oncology

University Medical Center Utrecht

Utrecht, the Netherlands

M. Maas

Department of Radiology

Antoni van Leeuwenhoek

The Netherlands Cancer Institute

Amsterdam, the Netherlands
G.J. Meijer

Department of Radiation Oncology

University Medical Center Utrecht

Utrecht, the Netherlands

\section{S. Mook}

Department of Radiation Oncology

University Medical Center Utrecht

Utrecht, the Netherlands

\section{J. Nijkamp}

Department of Radiation Oncology

Antoni van Leeuwenhoek

The Netherlands Cancer Institute

Amsterdam, the Netherlands

\section{M.E. Nowee}

Department of Radiation Oncology

Antoni van Leeuwenhoek

The Netherlands Cancer Institute

Amsterdam, the Netherlands

P.S.N. van Rossum

Department of Radiation Oncology

University Medical Center Utrecht

Utrecht, the Netherlands

P. Snaebjornsson

Department of Pathology

Antoni van Leeuwenhoek

The Netherlands Cancer Institute

Amsterdam, the Netherlands

J.W. van Sandick

Department of Surgery

Antoni van Leeuwenhoek

The Netherlands Cancer Institute

Amsterdam, the Netherlands 
S.T. van Turenhout

Department of Gastroenterology

Antoni van Leeuwenhoek

The Netherlands Cancer Institute

Amsterdam, the Netherlands

E. Vegt

Department of Nuclear Medicine

Antoni van Leeuwenhoek

The Netherlands Cancer Institute

Amsterdam, the Netherlands

M. Verheii

Department of Radiation Oncology

Antoni van Leeuwenhoek

The Netherlands Cancer Institute

Amsterdam, the Netherlands

F.E.M. Voncken

Department of Radiation Oncology

Antoni van Leeuwenhoek

The Netherlands Cancer Institute

Amsterdam, the Netherlands

F.J. Wessels

Department of Radiology

University Medical Center Utrecht

Utrecht, the Netherlands 
LIST OF CONTRIBUTING AUTHORS 


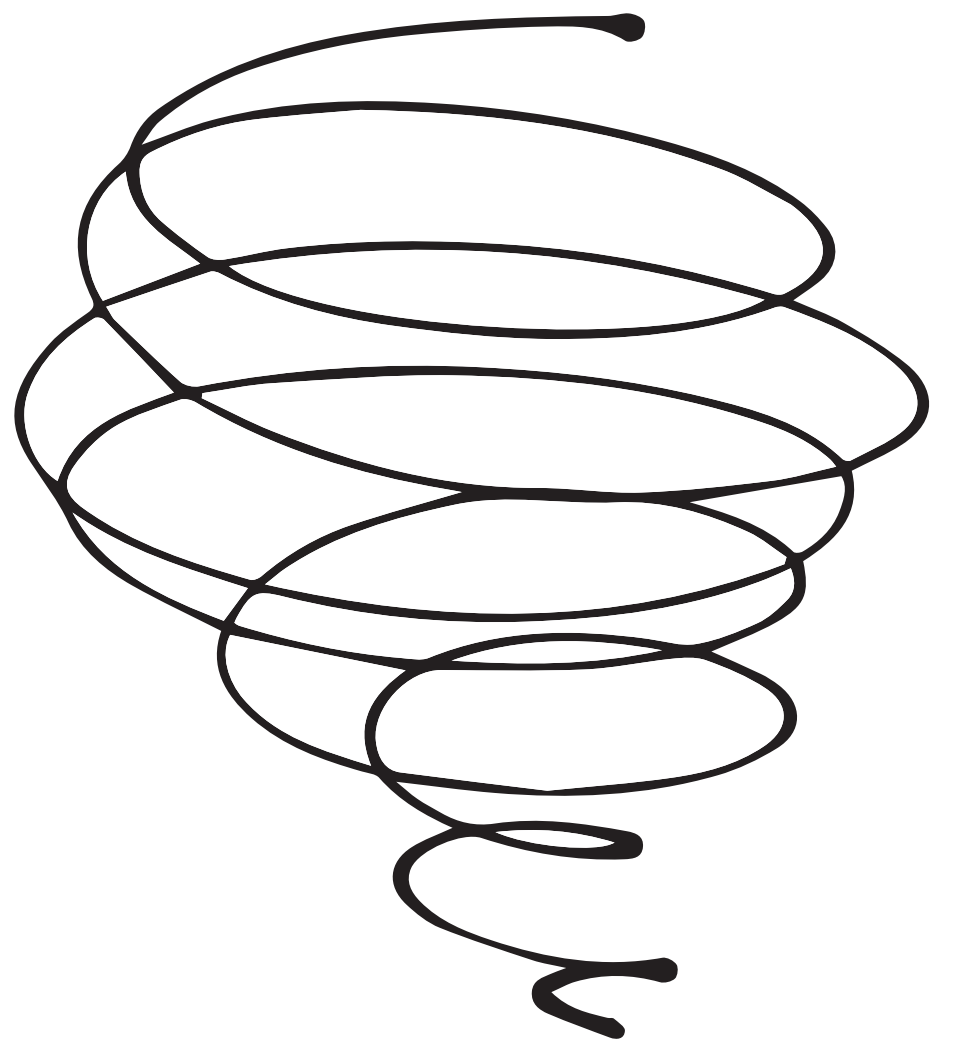


APPENDICES

Dankwoord 
En dan nu eindelijk het hoofdstuk dat door iedereen gelezen wordt.

Allereerst heel veel dank aan alle patiënten. Ik bewonder jullie bereidheid om meermaals vrijwillig naar het AvL te komen om in het lawaai van een MRI-scanner te gaan liggen en zelfs een extra endoscopie/EUS-onderzoek te ondergaan, puur om de patiënten 'na jullie' te kunnen helpen. De resultaten van deze onderzoeken helpen onze kennis over slokdarmkanker verder en jullie nuchtere instelling heeft mij ook persoonlijk gemotiveerd.

Mijn promotor, prof. dr. Beets-Tan, beste Regina, je enthousiasme voor de wetenschap en vertrouwen in de toekomst van de radiologie in een multidisciplinaire setting is inspirerend. Door je ervaring met de watch-and-wait voor patiënten met rectumcarcinoom kregen mijn projecten in sneltreinvaart richting. Dank voor je vertrouwen in mij.

Copromotoren, dr. Bartels-Rutten en dr. Voncken, lieve Annemarieke en Francine, steunpilaren, zonder jullie enthousiasme was dit proefschrift er niet geweest. Onze wekelijkse gezellige vrijdagmiddag overleggen en jullie $24 / 7$ bereikbaarheid hebben mij hierheen gebracht. Ik vind het knap hoe jullie de kliniek combineren met de wetenschap en ook nog een lief gezin. Dank voor jullie vertrouwen en de vrijheid die jullie me hebben gegeven om overal ter wereld aan mijn artikelen te kunnen werken. Ik heb ontzettend veel van jullie geleerd en jullie zijn voor mij allebei een groot voorbeeld.

Prof. dr. Masclee, Prof. dr. Bouvy, Prof. dr. Haustermans, Prof. dr. Stassen en Prof. dr. Stoker, hartelijk dank voor het beoordelen van mijn proefschrift. Prof dr. Wijnhoven, het is een eer dat u wilt plaatsnemen in de oppositie, ik heb veel van $u$ en uw onderzoeksgroep geleerd de afgelopen jaren en kijk vit naar mijn verdediging.

Slokdarm-maag-team van het AvL, dank voor de gezellige tijd en jullie kritische blik. Jullie hebben mijn presentaties op congressen tot een hoger niveau getild. Dr. van Dieren, lieve Jolanda, dank voor de gezellige dagen op de scopiekamer. Van goudmarkers tot bite-on-bite biopten en CRFs, geen enkel idee is jou te gek. Dr. van Sandick, lieve Johanna, dank voor je hulp bij mijn onderzoek en manuscripten, en natuurlijk ook voor de hulp bij mijn sollicitatie voor de chirurgie in het OLVG. Dr. Aleman, Berthe, dank voor je heldere blik op alle manuscripten en op mijn ontwikkeling vanuit de OOA-commissie. Dr. Hartemink, beste Koen, dank dat je plaats nam in mijn OOA-commissie en voor je betrokkenheid bij het onderzoek. Yvonne, je bent een grote aanwinst voor de patiënten maar ook voor het team. Ik vond het altijd heel leuk om met je te werken, dankjewel. Willem, immer collega, dank voor de gezellige samenwerking in het onderzoek en nu ook in de kliniek, "we moeten de buik in"! RTstudieondersteuners, clinical project managers en MRI-laboranten, dank voor de planning en organisatie. 
Beste mede-auteurs. Collega's vit UMC Utrecht, dank voor de samenwerking. Dr. Lambregts en dr. Maas, Doenja en Monique, veel dank voor jullie tijd bij het scoren van de MRI-scans en in het bijzonder voor jullie advies vanuit jullie ervaring bij het rectumcarcinoom. Dr. Nowee, beste Marlies, dank voor de samenwerking bij ons MR-intekenstuk. Dr. Ir. ter Beek, beste Leon, hartelijk dank voor je enthousiaste hulp bij de ontwikkeling van perfecte MRI-protocollen en de vele testavonden. Dr. Ir. Bartels, Wilbert, dank voor de leerzame MRI-cursus in Utrecht en voor je werk bij onze systematic review. Ook al is het geen 'original', ben ik misschien wel het meest trots op de manier waarop we de literatuur omtrent ADC in slokdarmcarcinoom hebben ontleed.

Lieve Paranimfen, classic, op moment van schrijven zijn jullie nog niet gevraagd dus hopelijk zeggen jullie ja... Philippe, volgens mij weten we inmiddels evenveel van slokdarmkanker want je hebt jarenlang naar mijn verhalen geluisterd. Geneeskunde hadden we bijna samen op Hawaii gedaan omdat daar geen loting was, maar gelukkig konden we alsnog op co-schap kitesurfen op Aruba gevolgd door een co-schap no-stress jungletrips in Suriname. Zelfs ons eigen bedriif in een andere 'branche' blijkt ineens een succes en samen een marathon rennen was eigenlijk niet een zwaar maar gewoon gezellig. Ik ben heel blij met jou als beste vriend. Brigit, op 23 oktober 2020 deed $\mathrm{jii}$ het voor, vanzelfsprekend heb je de lat op het dak gelegd. Toen je het tuinhuis binnenkwam wist ik meteen dat jii bij ons op de kamer moest komen. Je doorzettingsvermogen is aanstekelijk en we zaten altijd op 1 lijn. Je bent een loyale en lieve vriendin en een soort zus geworden door de jaren heen. Met jullie naast me kan er niks misgaan, op naar nog vele jaren vriendschap.

Dank aan alle onderzoekers uit het tuinhuis en het O-gebouw, die er tijdens de PhD retreat met "onze" DJ in Zeeland tot aan een wild weekend Antwerpen een mooie promotietijd van hebben gemaakt. Jorrita, de spil in het web van het tuinhuis, dankjewel. Marit, het was fijn om samen brainstormen over de overeenkomsten en verschillen tussen het rectum en de oesophagus onder het genot van cappuccino en (halve) gevulde koeken, dank voor de waardevolle vriendschap. Myrte, kort maar krachtig was onze tijd in het AvL en ik heb genoten van de bakken energie die iij altijd meebracht. Lisa, dank voor de gezellige tijd, voor je interesse in mijn onderzoek en voor de steun op ons allereerste ECR. Het weekend carnaval in Maastricht met de blondies zal ik nooit vergeten. Maurits, met jou een biertje drinken of op congres en alle zorgen verdwijnen. Dank voor je rust en intelligentie, je bent een trouwe vriend. Femke, spring in 't veld, dank voor je vrolijkheid en betrokkenheid. Rebecca, ik heb genoten van de gezelligheid tijdens ESGAR in Dublin samen.

Collega's, chirurgen en verpleegkundigen in het OLVG Oost, dank voor de kans om in zo een warm stadsziekenhuis de kliniek in te gaan en voor de gezelligheid tijdens alle diensten. Dank ook voor jullie steun en betrokkenheid bij mijn carrière, ik weet zeker dat we elkaar in de toekomst nog tegenkomen. 
Vrienden van Maarssen tot Utrecht, Leiden, Rotterdam en natuurlijk door heel Amsterdam, dank voor de waardevolle vriendschappen. Nadine, ik ken je al sinds mijn geboorte, altijd trouw en eerlijk, dank voor je humor en je interesse in alles wat ik doe. Emma, dank voor de bijzondere vriendschap die zelfs in stand bleef toen we nog maar in groep 4 zaten en iij naar Groningen verhuisde. Lisa en Boudewijn, de E\&M'ers die mij motiveerden om op de beste school van Nederland te blijven en nu zijn jullie vlammende advocaten van hier tot NYC. Dank voor de vele grote feesten, voor de slappe lach en de mooie vriendschap door dik en dun. Anna, vriendinnen sinds we samen de dakgoot schoonmaakten, dank voor je eerlijkheid, met jou is het altijd vertrouwd. Florine, dank voor je vastberaden interesse, steun en toeverlaat, als huisgenoot en nu ook vanuit Rotterdam, de stad met brede stoepen. Sophie-5, Charissa, Vivian en Doortje, jullie zijn unieke vriendinnen en we zullen dineren tot en met ons pensioen. Janine, Caro en Laura, dank voor de eiland-vriendschap van Aruba tot Wangerooge tot Ameland, snel weer surfen of op een steen zitten als dit boek gedrukt is. Lieve Wilma en Emile, dank voor jullie support. Wilma stuurde de vacature en nu is het afgerond, vanzelfsprekend in Hermès oranje.

Lieve schoonfamilie, Betty, Elka, Paul, Marischka, Kiki en Maarten, dank voor alle gezellige avonden, vitjes, goede gesprekken en de prachtige reizen. Veel dank ook voor jullie interesse in mijn onderzoek, carrière en eindeloze hobby's.

Lieve familie, oma Tini, dank voor de gezellige etentjes in Amstelveen en voor je scherpe blik. Deze oma kun je niet voor de gek houden, dit is de slimste van Nederland. Opa Johan en oma Lies, dank voor de warmte van de grote gezellige familie in Bergen. Lieve papa en mama, dank voor alles. Ik ben heel blij dat jullie me overal in steunen met als enige advies om zelf na te denken en met beide benen op de grond blijven staan. Het huis staat altijd open voor zoete inval en met de hondjes erbij is het feest compleet. Marc en Charlotte, dank voor jullie eerlijke adviezen. Marc, vlak voordat ik aan mijn promotie begon, zocht ik je op op Bali waar je met passie een hele specerijenfabriek optimaliseerde. lk was echt onder de indruk van hoe snel je Indonesisch sprak en hoe geliefd je was onder alle fabrieksmedewerkers. En Charlotte, je bent lief en (eigen)wijs, dank voor je heldere blik en voor je keiharde lachbuien met de tranen in je ogen. Het was te gek om door mijn kleine zusje door het grote Toronto geleid te worden en ik kan niet wachten om samen te dokteren. Michiel, dank voor je vrolijkheid, energie en loyaliteit.

Achter elke succesvolle vrouw staat een man die tonijnburgers bakt. "lk wíst al dat ze je zouden aannemen" waren je woorden nadat ik was aangenomen en waarschijnlijk zeg je vandaag "Ik wíst al dat het je zou lukken". Allerliefste Matthijs, top 1. Dank voor je adviezen die dit proefschrift perfect hebben gemaakt en voor je onvoorwaardelijke steun. Je geeft me het vertrouwen dat niks onmogelijk is. 
DANKWOORD 


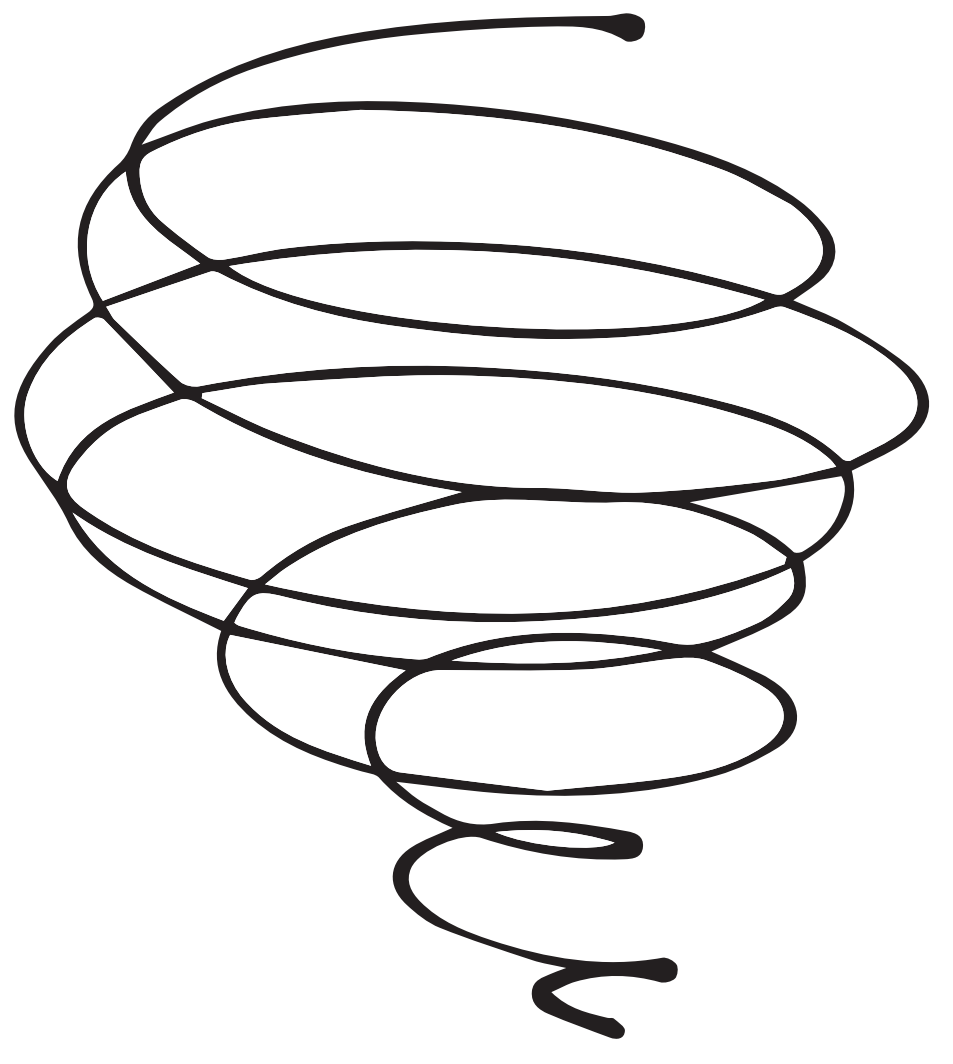


APPENDICES

Curriculum Vitae 
APPENDICES 


\section{CURRICULUM VITAE}

Sophie Elise Vollenbrock was born on September 9th, 1991, in Maarssen, the Netherlands. She graduated from secondary school at Christelijk Gymnasium Utrecht in 2009 and studied Biomedical Sciences at the Vrije Universiteit (VU) in Amsterdam. In 2010 she started studying medicine at the same university. Besides her study, she was involved in several committees at her student association and was treasurer of the board of the Sustainable Healthcare Challenge, focusing on organizing events to facilitate entrepreneurship in healthcare for students and young professionals. Sophie also went to Aruba for an internship at a general practitioner, where she enjoyed kitesurfing in the weekends. Thereafter,

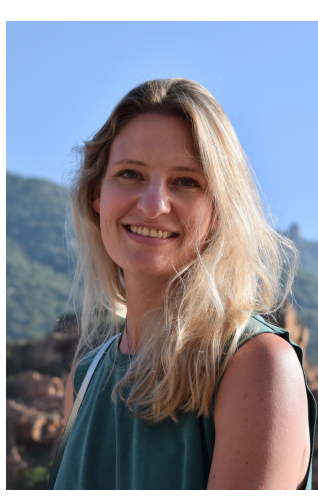
she went to Suriname for an internship in occupational medicine.

After graduating from medical school in 2017, Sophie started as a PhD candidate at the Netherlands Cancer Institute under the supervision of prof. dr. Beets-Tan, dr. Bartels-Rutten and dr. Voncken. She focused on oesophageal cancer patients and explored the role for clinical MRI assessment to improve the selection of complete responders after neoadjuvant chemoradiotherapy. Sophie presented at many national and international conferences and got awarded for 'Best Presentation' at the Dutch Congress of Radiology in 2018 and at the European Congress of Radiology (ECR) in 2018. As a hobby during her PhD, she started embroidering and in the summer of 2018, she and her best friend launched a fashion label called 'Maison Noir Amsterdam'. Since November 2019, Sophie is working as a surgical resident not in training at the Department of Surgery of Onze Lieve Vrouwe Gasthuis (OLVG), location Oost, Amsterdam, the Netherlands. 
